\title{
Fatty liver disease
}

Citation for published version (APA):

van de Wier, B. (2016). Fatty liver disease: from pathogenesis to pharmacotherapy. [Doctoral Thesis, Maastricht University]. https://doi.org/10.26481/dis.20160705bw

Document status and date:

Published: 01/01/2016

DOI:

10.26481/dis.20160705bw

Document Version:

Publisher's PDF, also known as Version of record

\section{Please check the document version of this publication:}

- A submitted manuscript is the version of the article upon submission and before peer-review. There can be important differences between the submitted version and the official published version of record.

People interested in the research are advised to contact the author for the final version of the publication, or visit the DOI to the publisher's website.

- The final author version and the galley proof are versions of the publication after peer review.

- The final published version features the final layout of the paper including the volume, issue and page numbers.

Link to publication

\footnotetext{
General rights rights.

- You may freely distribute the URL identifying the publication in the public portal. please follow below link for the End User Agreement:

www.umlib.nl/taverne-license

Take down policy

If you believe that this document breaches copyright please contact us at:

repository@maastrichtuniversity.nl

providing details and we will investigate your claim.
}

Copyright and moral rights for the publications made accessible in the public portal are retained by the authors and/or other copyright owners and it is a condition of accessing publications that users recognise and abide by the legal requirements associated with these

- Users may download and print one copy of any publication from the public portal for the purpose of private study or research.

- You may not further distribute the material or use it for any profit-making activity or commercial gain

If the publication is distributed under the terms of Article $25 \mathrm{fa}$ of the Dutch Copyright Act, indicated by the "Taverne" license above, 
FATTY LIVER DISEASE:

FROM PATHOGENESIS TO PHARMACOTHERAPY

Bregje van de Wier 
(C) Bregje van de Wier, Maastricht, 2016 ISBN: 978-94-6295-358-1

Cover design: Proefschriftmaken.nl || Uitgeverij BOXPress Layout: Bregje van de Wier Printed by: Proefschriftmaken.nl || Uitgeverij BOXPress

The research presented in this thesis was conducted at NUTRIM School of Nutrition and Translational Research in Metabolism which participates in the Graduate School VLAG (Food Technology, Agrobiotechnology, Nutrition and Health Sciences), accredited by the Royal Netherlands Academy of Arts and Sciences. 


\title{
Fatty liver disease:
}

\section{From pathogenesis to pharmacotherapy}

\author{
PROEFSCHRIFT
}

ter verkrijging van de graad van doctor aan de Universiteit Maastricht, op gezag van de Rector Magnificus, Prof dr. L.L.G. Soete, volgens het besluit van het College van Decanen, in het openbaar te verdedigen op dinsdag 5 juli 2016 om 12.00 uur

door

Bregje van de Wier

Geboren te Tilburg op 25 december 1985 


\section{Promotor}

Prof. dr. A. Bast

\section{Copromotores}

Dr. G.R.M.M. Haenen

Dr. G.H. Koek

\section{Beoordelingscommissie}

Prof. dr. O. Bekers (voorzitter)

Prof. dr. F.J.P.H. Brouns

Prof. dr. C. Neef

Prof. dr. F. Nevens (Universitair Ziekenhuis Leuven)

Prof. dr. F. Tacke (University Hospital Aachen) 


\section{Table of contents}

$\begin{array}{lll}\text { Chapter } 1 & \text { General introduction } & \mathbf{7}\end{array}$

Chapter 2 Elevated citrate levels in NAFLD: the potential of 29 citrate to promote radical production

Chapter 3 The potential of flavonoids in the treatment of NAFLD $\quad \mathbf{4 7}$

Chapter 4 The role of SOD2 in the development of NASH 85

Chapter 5 The flavonoid MonoHER promotes the adaptation to 101 oxidative stress during the onset of NAFLD

Chapter 6 Chemical characteristics for optimizing CYP2E1 115 inhibition

$\begin{array}{lll}\text { Chapter } 7 & \text { General discussion } & 131\end{array}$

$\begin{array}{lll}\text { Chapter } 8 & \text { Summary } & 145\end{array}$

$\begin{array}{ll}\text { Nederlandstalige samenvatting } & 151\end{array}$

$\begin{array}{ll}\text { Valorisatie addendum } & 157\end{array}$

$\begin{array}{ll}\text { Dankwoord } & 163\end{array}$

$\begin{array}{ll}\text { Curriculum Vitae } & 167\end{array}$

$\begin{array}{ll}\text { List of publications } & 169\end{array}$

$\begin{array}{ll}\text { List of abbreviations } & 171\end{array}$

Supplementary data $\quad \mathbf{1 7 5}$ 

Chapter 1

General introduction 


\section{Non-alcoholic fatty liver disease}

\section{Prevalence and risk factors}

Non-alcoholic fatty liver disease (NAFLD) was first described by Ludwig et al in 1980 as a liver disease resembling alcoholic liver disease, but seen in patients with no significant alcohol consumption [1]. The quantification of significant alcohol consumption varies. Many studies define significant alcohol consumption as $>20$ $\mathrm{g} /$ day in women and > $30 \mathrm{~g} /$ day in men; another definition, suggested by the American Gastroenterological Association and American College of Gastroenterology is $>21$ drinks per week in men and $>14$ drinks per week in women over a 2-year period [2]. Since 1980, the number of publications investigating NAFLD has substantially increased (figure 1).

\section{NAFLD publications}

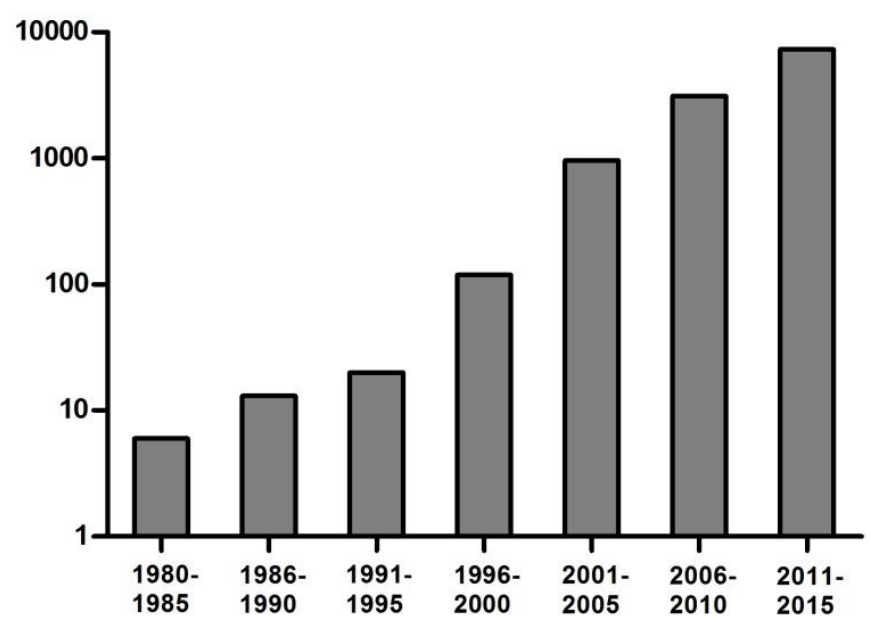

Figure 1. Number of publications found in PubMed investigating non-alcoholic fatty liver disease or nonalcoholic steatohepatitis over the years.

NAFLD includes a spectrum of liver disorders, ranging from steatosis to non-alcoholic steatohepatitis (NASH), fibrosis, cirrhosis and hepatocellular carcinoma. It is the most prevalent liver disorder worldwide with a prevalence of $20-30 \%$ in Western countries $[3,4]$. The prevalence is expected to increase, in parallel with that of obesity and diabetes [5]. In line with that, a recent study in the United States reported a prevalence of $46 \%$ in adults visiting the Brooke Army Medical Centre [6]. 
NAFLD is associated with the metabolic syndrome [7]. Over $90 \%$ of NAFLD patients has at least one feature of the metabolic syndrome and one third of patients has at least three different features [8]. The metabolic syndrome is a complex of risk factors related to cardiovascular disease and type 2 diabetes mellitus. It includes abdominal obesity, elevated triglycerides, reduced high density lipoprotein (HDL) cholesterol, hypertension and elevated fasting glucose [9]. NAFLD is more often found in patients with obesity and diabetes mellitus [5, 10]. Furthermore, NAFLD patients more often are obese and more often have diabetes than patients without NAFLD [6]. Besides obesity, insulin resistance and other components of the metabolic syndrome, male gender and age are additional risk factors for the development of NAFLD [10].

\section{Pathogenesis}

For years, NAFLD has been regarded as a two hit disease [11]. Steatosis was seen as the first hit, making the liver vulnerable for a second hit that would induce inflammation. This perspective has changed now: NAFLD is regarded as a multiple parallel hits disease [12]. These multiple hits can be roughly subdivided into metabolic abnormalities, oxidative stress and inflammatory mechanisms, influenced by genetic background and induced by the Western way of nutrition and a sedentary lifestyle (figure 2).

Some authors suggest that steatosis and NASH are two distinct conditions, since inflammation might precede steatosis in NASH and simple steatosis not often progresses to NASH [13]. However, since the progression from simple steatosis to NASH has been reported in several histological studies [14-18], it is more likely that steatosis and NASH are part of the same disease spectrum. In some cases of NASH, inflammation may precede steatosis, in other cases steatosis precedes inflammation and inflammation might be a consequence of an impaired anti-lipotoxic protection [12].

\section{Metabolic abnormalities}

Hepatic steatosis is the increased ( $>5 \%$ ) accumulation of fat in the liver, mainly in the form of triglycerides (TG), located in the cytoplasm of hepatocytes. Mechanisms leading to liver fat accumulation include: (1) high free fatty acids (FFA) supply to the liver due to increased lipolysis from visceral and subcutaneous adipose tissue and dietary fat intake, (2) low FFA oxidation in relation to the FFA supply, (3) high hepatic lipogenesis and (4) low hepatic excretion of very low density lipoprotein (VLDL) (figure 2) $[12,19]$. 
An important, if not the most important metabolic abnormality contributing to the development of steatosis and inflammation is insulin resistance (figure 2). Due to insulin resistance, lipolysis is not inhibited by insulin. The FFA that are released by lipolysis activate inflammatory pathways and stimulate ectopic fat deposition and further enhance insulin resistance [20]. Additionally, insulin resistance stimulates gluconeogenesis in hepatocytes and reduces glycogen formation. De novo lipogenesis is fuelled by high levels of glucose and insulin due to insulin resistance. This is regulated through activation of hepatic transcription factors such as sterol regulatory element binding protein-1c (SREBP-1c) and carbohydrate response element binding proteins (ChREBP), which stimulate lipogenic enzymes such as glucokinase (gk), fatty acid synthase (FAS) and acetyl-coenzyme A carboxylase (ACC) [20]. High levels of insulin might also inhibit VLDL assembly, since insulin decreases the synthesis and stability of apolipoprotein B, a component of VLDL [21].

FFA can stimulate apoptosis by the induction of several cell death receptors that promote the extrinsic apoptosis pathway and by the induction of mitochondrial toxicity that promotes the intrinsic apoptosis pathway [21].

\section{Inflammatory mechanisms}

Although the majority of NAFLD patients will have a benign, simple steatosis; in about $30 \%$ an inflammation in the liver with systemic effects develops. Several mechanisms activate pro-inflammatory pathways in patients with NAFLD. This leads to the release of chemokines, cytokines and other pro-inflammatory molecules as is clearly summarized in the review of Farrell et al [22]. It is out of the scope of this introduction to give a complete overview of the inflammatory mechanisms involved. Therefore, the most important mechanisms are highlighted.

Macrophage infiltration of mainly visceral adipose tissue leads to an inflammatory response of the adipose tissue and secretion of adipokines with pro-inflammatory and pro-fibrotic properties. This contributes to insulin resistance [23]. Adipose tissue inflammation is stimulated by several factors, including relative ischemia and the production of hypoxia inducible factor-1, specific gut microflora and microfloradependent inflammatory responses and hormones such as leptin [23]. An inflammatory response is also induced in the liver, leading to an excess of proinflammatory cytokines such as interleukin-6 (IL-6) and tumor necrosis factor- $\alpha$ (TNF- $\alpha$ ) in the liver and systemically [23]. 
The innate as well as the adaptive immune system contribute to the development and progression of NAFLD [24]. Innate immune cells in the liver include Kupffer cells and natural killer cells. When the liver is injured, other innate immune cells, such as neutrophils, leukocytes, monocytes and inflammatory macrophages are also recruited to the liver [24]. Adaptive immune cells that can contribute to liver injury include natural killer T cells, T cells and B cells. Sinusoidal endothelial cells and hepatic stellate cells also contribute to inflammation and fibrosis in the liver [24].

A characteristic of NASH is chronic lobular inflammation in the absence of a pathogen, also called sterile inflammation [21]. Damaged cells, necrotic cells and to a lesser extent apoptotic cells distribute molecules, called damage-associated molecular patterns (DAMPs), which trigger inflammation [25]. Cytosolic multiprotein complexes that are present in parenchymal and non-parenchymal liver cells, called inflammasomes, are regarded very important in the initiation of inflammation in NAFLD [21]. The inflammasome needs two signals: the first is activation through the nuclear factor kappa B (NF- $\kappa \mathrm{B})$ pathway, which increases transcription of components of the inflammasome. The second signal is coming from DAMPs. In response to DAMPs the components of the inflammasome are brought together [21]. When the inflammasome is assembled, caspase- 1 is activated, which leads to activation of the pro-inflammatory cytokines IL-1 $\beta$ and IL-18 and neutralization of IL-33 which directs Th2 responses [26]. Although the detection of DAMPs and activation of inflammasomes can occur in several cell types in the liver, the Kupffer cells appear to be crucial in the development of NASH [21]. Kupffer cells might also be activated by dietary fat or oxidized low density lipoprotein (LDL) cholesterol [27, 28]. In addition, Kupffer cells possibly stimulate insulin resistance, since depletion of Kupffer cells attenuates insulin resistance in mouse studies [29, 30].

Furthermore, inflammatory pathways in the liver are stimulated by FFA and oxidative stress. Pathways that are activated by oxidative stress include the c-Jun N-terminal kinase and NF- $\kappa B$ pathway [23].

Another important facet contributing to NAFLD disease progression is endoplasmic reticulum (ER) stress, caused by lipid accumulation in the liver [21, 31]. ER stress was first observed in genetic and diet induced models of NASH, but was later also reported in NAFLD and NASH patients [31]. Signalling pathways that are induced by ER stress contribute to lipotoxicity, insulin resistance, inflammation, oxidative stress and apoptotic cell death [31]. 


\section{Oxidative stress}

Oxidative stress is caused when the production of reactive oxygen species (ROS) is not balanced by the protection against ROS by antioxidants. Several sources of oxidative stress can be identified in NAFLD [32-34]. Firstly, ROS are produced during the mitochondrial and peroxisomal beta-oxidation of FFA [32] and during the metabolism of FFA by cytochrome P450 2E1 (CYP2E1) [32, 35, 36]. Mitochondrial dysfunction is regarded to have a significant role in the pathogenesis of NAFLD and NASH [34]. Although other organelles contribute to the production of ROS, mitochondria are the main source of ROS production in hepatocytes [34]. Because $\beta$-oxidation is increased, the electron flux through the mitochondrial electron transport chain (ETC) is increased [37]. However, the ETC is not perfectly isolated from oxygen: at complexes I and III of the ETC some electrons directly react with oxygen, forming the superoxide anion radical $\left(\mathrm{O}_{2}{ }^{\circ}\right)$, which also generates other ROS and reactive nitrogen species [38]. In NASH, mutations in ETC complex II and decreased activity in all ETC complexes are reported [37]. It is hypothesized that electron leakage is further increased by the reduced activity of the ETC complexes: instead of electrons shuttling through cytochrome c oxidase and combining with protons and oxygen to form water, electrons directly react with oxygen and form ROS [37]. In NAFLD components of the ETC can be blocked by reactive lipid peroxidation products, ROS, reactive nitrogen species and TNF- $\alpha$, further increasing mitochondrial ROS production [39]. The produced ROS damage mitochondrial DNA (mt-DNA), which could decrease the transcription of mt-DNA encoded respiratory chain polypeptides, further contributing to the blockage of the electron flow in the ETC and increasing the production of ROS [38].

A decrease in antioxidant defences also contributes to oxidative stress. A lower glutathione level was found in the livers of patients with steatosis and NASH [40]. Reduced activities of superoxide dismutase (SOD), catalase (CAT) and glutathione peroxidase (GPx) were also reported in NASH patients, and were correlated to disease severity [33, 40]. Furthermore, hepatic gene expression of SOD1 and CAT were found to be reduced in NASH patients [41]. The ROS that are generated in NAFLD and that are not adequately scavenged by antioxidants, react with biological compounds including fatty acids, proteins and DNA, causing lipid peroxidation, stellate cell activation, inflammation (via NF- $\mathrm{BB}$ activation), ER stress, apoptosis and the previously described mitochondrial dysfunction [32, 34]. 
Iron is also linked to the pathogenesis of NAFLD. Elevated hepatic iron levels have been found in patients with NAFLD $[42,43]$. Although the exact role of iron in the pathogenesis of NAFLD is not yet clear, it is well known that iron can increase oxidative stress, for example by its ability to generate hydroxyl radicals via the Fenton reaction [44].

\section{Liver-gut axis}

The liver-gut axis is described separately, because it influences metabolic abnormalities as well as inflammatory mechanisms and oxidative stress [45]. The main interaction between gut and liver is the filtering of blood form the intestines via the vena portae by the liver; therefore it is not unrealistic that the gut microbiota play a role in the pathogenesis of NAFLD. The composition of microbiota differs between obese and lean individuals and also between NAFLD patients and healthy controls [46, 47]. Altered intestinal microbiota appear to increase liver fat via several mechanisms: by altered appetite signalling, increased energy extraction from the diet, changed expression of de novo lipogenesis genes and $\beta$-oxidation genes and by the induction of inflammation [47]. It is hypothesized that increased intestinal permeability leads to a translocation of bacteria and bacterial components to the circulation, inducing inflammation [47]. The bacterial products can activate specific toll-like receptors (TLRs) that are present in many different liver cells. Activation of TLRs in Kupffer cells induces inflammation, while activation of TLRs in hepatic stellate cells stimulates fibrosis [46]. Fibrosis can also be induced by the direct activation of hepatic stellate cells by lipopolysaccharide (LPS), a component of the cell wall of gram negative bacteria [47]. Lastly, activation of TLRs by bacterial products appears to stimulate insulin resistance and oxidative stress [46]. 


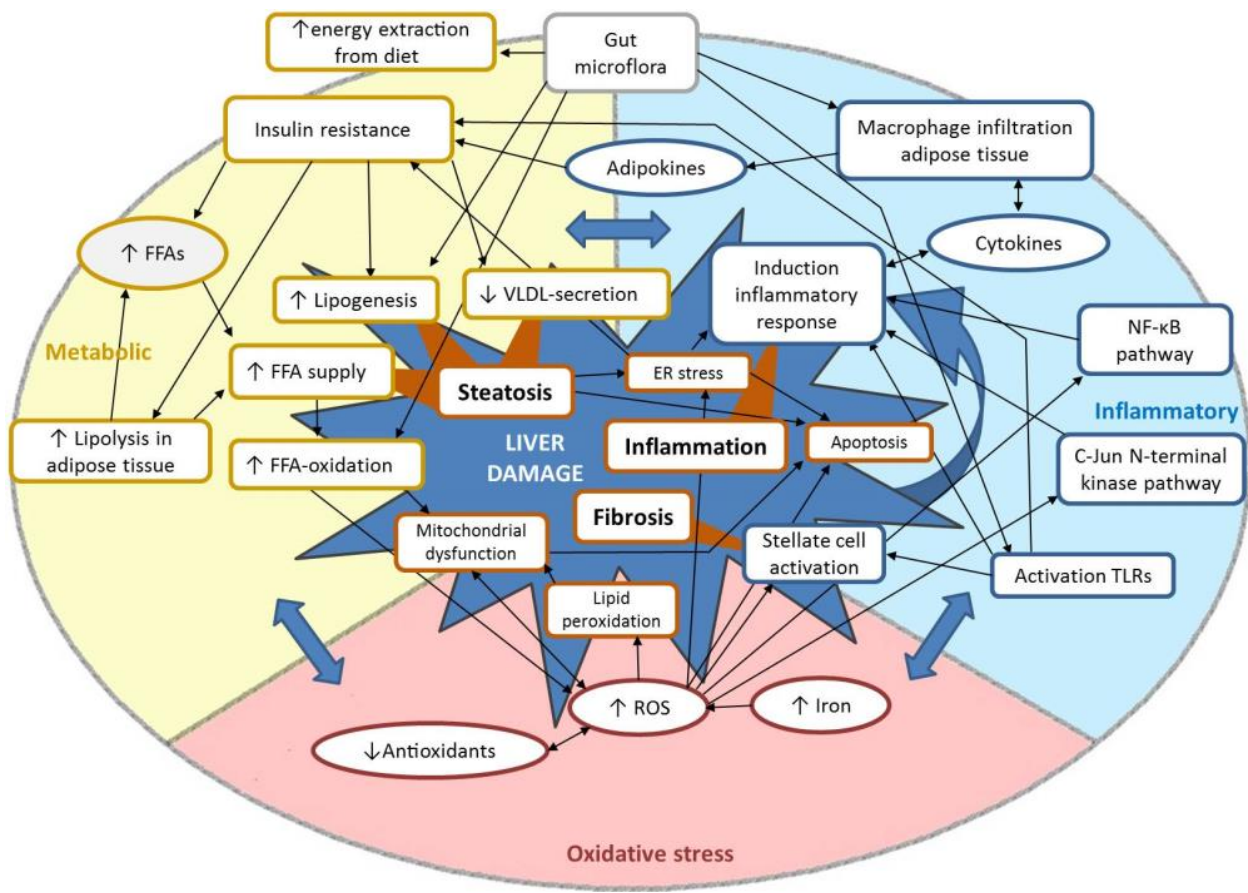

Figure 2. Schematic presentation of the complex interplay between the previously described metabolic (yellow), inflammatory (blue) and oxidative stress (red) pathways leading to liver damage (orange boxes) in NAFLD and NASH, with the most important hallmarks steatosis, inflammation and fibrosis. The inner circle represents processes in the liver, while in the outer circle also processes in the rest of the body (e.g. adipose tissue, gut) are displayed.

\section{Clinical features and diagnosis}

Most patients with NAFLD are asymptomatic or have non-specific symptoms such as fatigue. Some patients report pain in the abdominal upper right quadrant, probably as a consequence of liver swelling. On physical examination, abdominal obesity and hepatomegaly are commonly found [48]. There are no other signs that indicate the presence of NAFLD. Therefore, NAFLD is often incidentally found when patients undergo testing for other, unrelated symptoms or conditions.

NAFLD is the most common cause of elevated aminotransferases [5]. However, only about $50 \%$ of patients with simple steatosis have abnormal liver chemistry tests and about $80 \%$ of patients with advanced NAFLD have abnormal liver tests [49]. Laboratory findings do not correlate with the histological severity of the disease, 30 $60 \%$ of patients with biopsy proven NASH have normal alanine aminotransferase (ALT) levels [48]. Elevated glucose levels and dyslipidaemia are found in $30-50 \%$ of NAFLD patients and in $20-50 \%$ of patients elevated ferritin levels are found [49]. 
Various diagnostic panels have been suggested for the non-invasive diagnosis of NAFLD [50]. The SteatoTest is a combination of tests: the Fibrotest, which predicts the degree of fibrosis with the use of fibrosis biomarkers, combined with the ActiTest, which measures the degree of necroinflammatory activity of viral origin, and body mass index (BMI), serum cholesterol, triglycerides and glucose adjusted for age and gender [50]. The SteatoTest has a sensitivity between $45-95 \%$ and a specificity between $45-89 \%$ for the diagnosis of steatosis, depending on the cut-off value that is used (decreasing sensitivity and increasing specificity with a higher cut-off value) [50]. Another test used for the non-invasive diagnosis of steatosis is the fatty liver index (FLI). The FLI is a complex algorithm, based on the BMI, waist circumference and serum triglycerides and gamma-glutamyl transpeptidase $(\gamma \mathrm{GT})$. This test has a sensitivity of $87 \%$ and a specificity of $64 \%$ in patients with elevated ALT and $\gamma \mathrm{GT}$. Other non-invasive tests and algorithms have been developed, also for the diagnosis of NASH and fibrosis. The most promising test for the diagnosis of NASH appears to be detection of cytokeratin 18 (CK18) fragments, which are markers of hepatocyte apoptosis [51]. However, none of these tests have yet been recommend for use in clinical practice [2].

Liver biopsy is currently the gold standard for the diagnosis of NAFLD [52]. With liver biopsy, other causes of liver damage can be excluded and the severity of steatosis, inflammation and fibrosis can be estimated [53]. Unfortunately, it is an invasive procedure with potential risks and complications. Additionally, only a small portion (one $50.000^{\text {th }}$ ) of the liver parenchyma is examined and the diagnosis depends on the subjective judgment of the pathologist $[50,54,55]$. The histological NAFLD activity score (NAS) was primarily developed to monitor the effect of therapeutic interventions [56], though many studies also use it for the diagnosis of NASH. For diagnosing steatosis, ultrasound is a non-invasive alternative with a sensitivity of 60$94 \%$ and a specificity of $66-97 \%$ [51]. However, ultrasound has a low sensitivity for mild steatosis and it is not possible to differentiate between mild fibrosis and steatosis with ultrasound. Other drawbacks are the limited applicability of ultrasound for obese patients and an inter-observer agreement of only $72 \%$ [50, 57]. Computed tomography (CT) is not more accurate than ultrasound for the diagnosis of steatosis. Furthermore, it is more expensive and it exposes patients to radiation. Therefore, CT is not recommended [21]. Another alternative for identification of hepatic steatosis is magnetic resonance imaging (MRI). MRI has the advantage over ultrasound in detecting mild steatosis. Disadvantages of MRI include that it is expensive and not widely available $[50,58]$. Several MRI techniques can be used. The magnetic resonance spectroscopy (MRS), has demonstrated significant accuracy in diagnosing and quantifying steatosis with a sensitivity of $92-100 \%$ and a specificity of $92-97 \%$ [51]. However, MRS is also expensive and not widely available. A recently developed 
method to evaluated steatosis is the controlled attenuation parameter (CAP) [51]. Attenuation in the liver is measured using signals acquired by an elastography probe (FibroScan $囚$ ). An advantage of this method is that it is not influenced by fibrosis. Furthermore, CAP appears to be more sensitive than ultrasound for low grade steatosis, it is easy to perform, the results are operator independent and are objectively interpreted [21,51]. Also, it is less susceptible than liver biopsy for sampling error since it assesses 100 times more tissue [51]. Imaging techniques cannot be used for the diagnosis of NASH.

\section{Current treatment strategies}

To date, there are no licensed, evidence-based therapies for NAFLD [59]. Strategies for the treatment of NAFLD can be divided in three categories: lifestyle changes, pharmacotherapy and surgical interventions [59]. Diet and exercise are considered the first line of therapy in NAFLD. Weight loss of five percent or more, achieved by diet or exercise, has been shown to reduce hepatic steatosis. However, this is difficult to achieve for many patients. Furthermore, the effects on inflammation and fibrosis have not yet been adequately investigated [60-62].

Pharmacotherapy could be a method to reduce inflammation and fibrosis and would be a way to treat patients in an ad-on setting next to the lifestyle interventions. Several drugs have been investigated focused on specific pathological pathways.

Orlistat, an anti-obesity drug that inhibits enteric fat absorption, might stimulate limited weight reduction in selected patients [62]. Although it improved fatty liver on ultrasound in some NAFLD patients in one study, it did not improve liver histology in another $[63,64]$. Other drugs, proposed for the treatment of NAFLD, are drugs influencing the endo-cannabinoid system. Cannabinoid receptors type-1 (CB1 receptors) are expressed in hepatocytes and hepatic myofibroblasts and are involved in high fat storage, alcohol induced steatosis, liver regeneration and fibrogenesis. Cannabinoid receptor type-2 (CB2 receptor) activation might be anti-inflammatory and anti-fibrotic [65]. CB1 receptor antagonists and CB2 receptor agonists seem to be promising for the treatment of liver diseases [59, 65]. Rimonabant, an extensively investigated CB1 antagonist, was found to improve HDL-cholesterol, triglycerides, insulin resistance, waist circumference, visceral fat and liver fat [66, 67]. Unfortunately, rimonabant was found to have severe psychiatric side effects and was withdrawn from the market. CB1 antagonists that act peripherally, are expected to lack this side effect and should be investigated further [59]. No CB2 agonists have yet been developed for clinical use [65]. 
Anti-diabetic drugs have been widely studied for the treatment of NAFLD. Metformin, an oral blood glucose lowering drug, can improve biochemical and metabolic features, but it has not yet been proven to have an effect on liver histology [68]. Various studies show contra dictionary results and additional larger randomized controlled trials are needed [69]. Glitazones (TZDs), another class of oral blood glucose lowering drugs and agonists of the peroxisome proliferator-activated receptor $\gamma$ (PPAR- $\gamma$ ), which is involved in adipogenesis and glucose homeostasis, are also extensively investigated in NAFLD. Due to hepatic and cardiac toxicity, rosiglitazone and troglitazone were withdrawn from the market and research has now focused on pioglitazone. Studies have demonstrated that pioglitazone improves steatosis and inflammation in NASH patients. It might also improve fibrosis, but further studies are needed [70]. AASLD guidelines recommend that pioglitazone can be used in the treatment of biopsyproven NASH, with the notion that the majority of patients that participated in clinical trials was non-diabetic and that the long term safety and efficacy of pioglitazone has not yet been established [2]. Observed side effects such as weight gain, bone loss, congestive heart failure and bladder cancer, have to be considered when starting therapy with pioglitazone for longer duration [71]. New anti-diabetic drugs, GLP-1 receptor agonists and DPP-4 inhibitors, have shown promising results in animal and human NAFLD studies [59]. Two human studies on GLP-1 agonists liraglutide and exenatide showed improvement of transaminases and liver histology [72, 73]. Studies on the DPP-4 inhibitor sitagliptin showed improvement of transaminases, but conflicting results regarding liver histology [71, 72, 74, 75]. Further studies are needed to establish the benefit of new anti-diabetic drugs in diabetic and non-diabetic NAFLD patients [71].

Statins, fibrates and other lipid lowering agents have been studied for the treatment of NAFLD and NASH. A review of various human studies, including pilot, prospective, preliminary and post hoc analysis studies, that investigated lipid lowering agents for the treatment of NAFLD, concluded that lipid lowering agents are safe and efficacious in patients with NAFLD/NASH [76]. However, in a more recent systematic review of randomized controlled trials investigating statins in NAFLD, only two studies, which both had high risk of bias and small number of participants, could be included. It was found that statins might improve serum aminotransferase levels and ultrasound findings, but effects on liver histology were not evaluated [77]. Good quality RCT's of adequate size and duration are needed to establish the suitable lipid lowering treatment for hyperlipidemic and non-hyperlipidemic NAFLD patients [59].

It has been reported that omega-3 fatty acids have beneficial effects on lipid metabolism and inflammation and they are therefore studied for the treatment of NAFLD and NADH [78]. Supplementation of omega-3 polyunsatured fatty acid has been found to decrease liver fat in NAFLD, as reported in the systematic review of 
Parket et al. [79]. However, no reduction of aminotransferase levels was observed and the effect of omega-3 fatty acids on inflammation was not examined. It was concluded that further studies are needed to establish the optimal dose [79]. Also the effect of omega-3 fatty acids on histology, in particular on inflammation, has to be investigated. Two relatively recent randomized controlled trials did not find an improvement of liver histology in subjects with NASH supplemented with omega-3 fatty acids [80, 81]. Another recent randomized controlled trial did also not find a significant effect of omega-3 fatty acids on liver fat and liver fibrosis in patients with NAFLD [82].

Ursodeoxycholic acid (UDCA) decreases cholesterol secretion in bile. Therefore, it has been investigated for the treatment of NAFLD and NASH. Interestingly, it also appears to act on different mechanisms involved in the transition of steatosis to NASH in murine models [83]. In human studies, UDCA was found to improve liver function tests. In a few studies, steatosis and fibrosis were also improved. However, due to limited quality of the studies and heterogeneity of the results, further studies are needed to support the use of UDCA in NAFLD/NASH [84].

Based on the hypothesis that oxidative stress plays a key role in the progression from steatosis to NASH, antioxidants have also been extensively investigated for the treatment of NAFLD and NASH. The most often investigated antioxidant, and the only antioxidant recommended in the treatment of biopsy-proven NASH (in non-diabetic patients) according to AASLD guidelines, is vitamin E [2]. Vitamin E (in the form of RRR $\alpha$-tocopherol) in a dose of 800 IU daily is found to decrease aminotransferases, and improve steatosis and inflammation in NASH. It does not have an effect on fibrosis $[2,85]$. Nevertheless, the use of vitamin $\mathrm{E}$ is still debated, because some meta-analyses have found an increase in all-cause mortality with the use of vitamin E [86, 87], a finding that was not confirmed in later studies [88-90]. A dose response analysis in the study of Miller et al. demonstrated increased mortality risk with dosages greater than 150 IU daily [86]. However, the later studies that investigated dosages up 5000 IU vitamin E daily could not confirm this [88, 89]. Provisionally, the use of vitamin E is not recommended in diabetic NASH patients, NAFLD without liver biopsy and cirrhotic NASH [9].

Since intestinal microbiota have been suggested to play a role in the pathophysiology of NAFLD, recent research has also focused on the use of probiotics in NAFLD [91]. Small studies have observed beneficial effects of probiotics in NAFLD [92-94]. However, reviews of probiotics studies suggest that the use of probiotics cannot yet be recommended until larger high-quality randomized controlled trials have confirmed the beneficial effects [92, 94-96]. 
A surgical intervention in the treatment of NAFLD and NASH is bariatric surgery in patients with severe obesity (BMI>35). Although meta-analyses report that bariatric surgery improves or resolves steatosis, steatohepatitis and fibrosis [97, 98], no definitive judgment on the use of bariatric surgery can be made yet due to the lack of randomized controlled trials [99]. Liver transplantation is the only option in NAFLD patients that progress to end stage liver disease. The number of liver transplantation waitlist registrants with NAFLD increased largely over the last years. NAFLD is now the second-leading disease, after hepatitis $\mathrm{C}$, of patients awaiting liver transplantation [100]. Unfortunately, NAFLD can reoccur after transplantation [101].

Table 1. Most important investigated pharmacological treatments for NAFLD and NASH

\begin{tabular}{|c|c|c|c|c|}
\hline Group & Drug & $\begin{array}{l}\text { Mechanism of } \\
\text { action }\end{array}$ & Results & Conclusion \\
\hline \multirow{2}{*}{$\begin{array}{c}\text { Anti-obesity } \\
\text { drugs }\end{array}$} & Orlistat & $\begin{array}{l}\text { Inhibition of enteric } \\
\text { fat absorption }\end{array}$ & $\begin{array}{l}\text { Controversial results in } \\
\text { studies: improvement } \\
\text { of steatosis vs no } \\
\text { improvement of liver } \\
\text { histology }\end{array}$ & $\begin{array}{l}\text { More studies } \\
\text { needed }\end{array}$ \\
\hline & Rimonabant & $\begin{array}{l}\text { CB1-receptor } \\
\text { antagonist. } \\
\text { Improvement of } \\
\text { lipid metabolism an } \\
\text { inhibition of } \\
\text { fibrogenesis }\end{array}$ & $\begin{array}{l}\text { Improvement of HDL, } \\
\text { TG, IR, waist } \\
\text { circumference, visceral } \\
\text { fat and liver fat, but } \\
\text { severe psychiatric side } \\
\text { effects. }\end{array}$ & $\begin{array}{l}\text { Withdrawn. New } \\
\text { studies on } \\
\text { peripherally } \\
\text { restricted CB1 } \\
\text { antagonists. }\end{array}$ \\
\hline \multirow[b]{2}{*}{$\begin{array}{c}\text { Anti-diabetic } \\
\text { drugs }\end{array}$} & Metformin & $\begin{array}{l}\text { Decrease of blood } \\
\text { gluc via different } \\
\text { mechanisms }\end{array}$ & $\begin{array}{l}\text { Improvement of } \\
\text { biochemical and } \\
\text { metabolic features but } \\
\text { no improvement of liver } \\
\text { histology }\end{array}$ & $\begin{array}{l}\text { Additional larger } \\
\text { good quality } \\
\text { RCT's needed }\end{array}$ \\
\hline & Glitazones & $\begin{array}{l}\text { PPAR- } \gamma \text { agonist. } \\
\text { Improves insulin } \\
\text { sensitivity of fat } \\
\text { tissue, skeletal } \\
\text { muscles and liver. }\end{array}$ & $\begin{array}{l}\text { Improvement of } \\
\text { steatosis and } \\
\text { inflammation in NASH } \\
\text { patients, but } \\
\text { considerable side } \\
\text { effects. Hepatic and } \\
\text { cardiac toxicity of } \\
\text { rosiglitazone and } \\
\text { troglitazone. }\end{array}$ & $\begin{array}{l}\text { Pioglitazone is } \\
\text { used in NASH } \\
\text { patients. Long } \\
\text { term safety and } \\
\text { efficacy have to } \\
\text { be evaluated. } \\
\text { Rosiglitazone } \\
\text { and troglitazone } \\
\text { withdrawn. }\end{array}$ \\
\hline
\end{tabular}




\begin{tabular}{|c|c|c|c|c|}
\hline $\begin{array}{l}\text { Anti-diabetic } \\
\text { drugs } \\
\text { (continued) }\end{array}$ & $\begin{array}{l}\text { Liraglutide } \\
\text { and } \\
\text { exenatide }\end{array}$ & $\begin{array}{l}\text { GLP-1 agonists. } \\
\text { Improvement of } \\
\text { gluc homeostasis. } \\
\text { DPP-4 inhibitor. } \\
\text { Inhibition of GLP-1 } \\
\text { degradation and } \\
\text { improvement of } \\
\text { gluc homeostasis. }\end{array}$ & $\begin{array}{l}\text { Improvement of } \\
\text { transaminases and liver } \\
\text { histology. } \\
\text { Improvement of } \\
\text { transaminases, but } \\
\text { conflicting results on } \\
\text { liver histology. }\end{array}$ & $\begin{array}{l}\text { More studies } \\
\text { needed. }\end{array}$ \\
\hline \multirow{3}{*}{$\begin{array}{l}\text { Lipid lowering } \\
\text { agents }\end{array}$} & Statins & $\begin{array}{l}\text { Inhibition of } \\
\text { cholesterol } \\
\text { synthesis. }\end{array}$ & $\begin{array}{l}\text { Improvement of } \\
\text { transaminases and } \\
\text { ultrasound findings, but } \\
\text { effect on histology } \\
\text { unknown. }\end{array}$ & $\begin{array}{l}\text { Good quality } \\
\text { RCT's needed. }\end{array}$ \\
\hline & Fibrates & $\begin{array}{l}\text { Decrease of lipids } \\
\text { via several } \\
\text { mechanisms. } \\
\text { Activation of PPAR- } \\
\alpha .\end{array}$ & $\begin{array}{l}\text { Controversial results on } \\
\text { transaminases: } \\
\text { improvement or no } \\
\text { effect. No effect on } \\
\text { histology. Improvement } \\
\text { of fasting gluc. }\end{array}$ & $\begin{array}{l}\text { Additional larger } \\
\text { good quality } \\
\text { RCT's needed. }\end{array}$ \\
\hline & $\begin{array}{l}\text { Omega-3- } \\
\text { fatty acids }\end{array}$ & $\begin{array}{l}\text { Naturally occurring } \\
\text { ligands of PPAR- } \alpha \\
\text { that modulate lipid } \\
\text { metabolism. }\end{array}$ & $\begin{array}{l}\text { No effect on } \\
\text { transaminases. } \\
\text { Controversial results on } \\
\text { histology: decrease of } \\
\text { liver fat or no effect. }\end{array}$ & $\begin{array}{l}\text { Additional good } \\
\text { quality and long- } \\
\text { term RCT's } \\
\text { needed. }\end{array}$ \\
\hline Bile acids & $\begin{array}{l}\text { Ursodeoxy- } \\
\text { cholic acid }\end{array}$ & $\begin{array}{l}\text { Decrease of } \\
\text { cholesterol } \\
\text { secretion in bile. }\end{array}$ & $\begin{array}{l}\text { Improvement of liver } \\
\text { function tests. } \\
\text { Improvement of } \\
\text { steatosis and fibrosis in } \\
\text { a few studies. }\end{array}$ & $\begin{array}{l}\text { Larger good } \\
\text { quality RCT's } \\
\text { needed. }\end{array}$ \\
\hline Antioxidants & Vitamin $\mathrm{E}$ & $\begin{array}{l}\text { Scavenger of } \\
\text { reactive oxygen } \\
\text { species. }\end{array}$ & $\begin{array}{l}\text { Improvement of } \\
\text { transaminases, } \\
\text { steatosis and } \\
\text { inflammation. No effect } \\
\text { on fibrosis. }\end{array}$ & $\begin{array}{l}\text { Recommended } \\
\text { in biopsy-proven } \\
\text { NASH in non- } \\
\text { diabetic patients. }\end{array}$ \\
\hline Probiotics & $\begin{array}{l}\text { Various } \\
\text { probiotic } \\
\text { formulas }\end{array}$ & $\begin{array}{l}\text { Restoration of } \\
\text { gastro-intestinal } \\
\text { barrier function, } \\
\text { modulation of } \\
\text { immune function } \\
\text { and inhibition of } \\
\text { proliferation of } \\
\text { harmful bacteria. }\end{array}$ & $\begin{array}{l}\text { Improvement of } \\
\text { transaminases, total } \\
\text { and HDL cholesterol } \\
\text { and insulin resistance. } \\
\text { Improvement of } \\
\text { steatosis in one small } \\
\text { study. }\end{array}$ & $\begin{array}{l}\text { Larger good } \\
\text { quality } \mathrm{RCT}^{\prime} \text { s } \\
\text { needed. }\end{array}$ \\
\hline
\end{tabular}

Gluc = glucose, $\mathrm{CB} 1=$ cannabinoid receptor $1, \mathrm{HDL}=$ high density lipoprotein cholesterol, $\mathrm{TG}=$ triglycerides, IR = insulin resistance, PPAR- $\gamma=$ proliferator receptor $\gamma$, GLP-1 = glucagon-like-peptide- 1 , DPP- $4=$ Dipeptidylpeptidase-4, 


\section{Prognosis}

NAFLD is a slowly progressing disease. In some patients no progression is seen for years and in some patients the disease may regress [101]. Although various studies have reported the progression of simple steatosis to NASH [14-18], it is not completely clear how many patients progress from simple steatosis to NASH. The risk of a patient with simple steatosis to eventually develop cirrhosis, has been estimated to be $1-2 \%$ [102]. The risk of patients with NASH to eventually develop cirrhosis is higher and varies from $0 \%$ at 5 years to $12 \%$ at 8 years $[103,104]$. NASH progression to cirrhosis has also been associated with an increased risk for the development of hepatocellular carcinoma (HCC) [105].

The survival of patients with NAFLD is found to be lower compared to the general population. Leading causes of dead are cardiovascular- and cancer-related, followed by liver-related causes [106-109]. Liver related mortality is increased in NASH patients compared to NAFLD patients. Liver related mortality was found to be $11 \%$ in NASH patients and 2\% in non-NASH NAFLD patients after 8 years [103]. After 18.5 years this increased to $18 \%$ for NASH patients and $3 \%$ for non-NASH NAFLD patients [106]. Therefore it is of the utmost importance to decrease the NAFLD epidemic that is strongly correlated to overweight and obesity caused by the Western way of life.

\section{Aim and outline of the thesis}

In this thesis we will try to further elucidate the pathogenesis of NAFLD and NASH, with emphasis on the processes/mechanisms of oxidative stress and inflammation. We will examine if the processes of oxidative stress and the onset of inflammation can be adequately represented in an animal model. Eventually, we investigate new treatment strategies for NAFLD and NASH.

In chapter two the potential role of citrate in NAFLD is investigated. Elevated plasma citrate levels were demonstrated in patients with NAFLD. Cellular experiments were performed to investigate if elevated citrate levels can originate from an excessive supply of fatty acids to liver cells. Furthermore, the effect of citrate on oxidative stress was examined.

In chapter three, a concise overview of the pathogenesis of NAFLD is given. It introduces flavonoids as potential therapeutic agents in the treatment of NAFLD and reports on how flavonoids can affect various pathways in this multi-faceted disease. 
Animal models used in studies that examine flavonoids in the treatment of NAFLD are evaluated. The results of these studies are reviewed, proposing rutin and its derivates, such as monoHER for further investigation in NAFLD.

In chapter four, three different mice models to study the onset of inflammation in NAFLD are compared. It was hypothesized that in combination with a high fat diet, complete knockout of the mitochondrial antioxidant SOD2, would lead to a rigorous increase in oxidative stress in the liver of these mice, which would induce inflammation. The liver specific SOD2 knockout mice on a high fat diet were compared to LDL-receptor knockout mice, which are known to develop inflammation on a high fat diet, and to wildtype mice, which are known to only develop steatosis after a few weeks of high fat diet.

The effect of the flavonoid monoHER on the development of steatohepatitis was examined in LDL-receptor knockout mice in chapter five. In this chapter we focused on the effect of monoHER on oxidative stress and the adaptive response to oxidative stress in NAFLD. This was assessed at three levels: the direct damage by ROS, the nuclear factor erythroid derived 2 (NRF2) induced gene expression and the adaptive response on cellular redox status.

CYP2E1 is implicated in the pathogenesis of NAFLD and alcoholic liver disease by the production of ROS and the formation of toxic metabolites. In chapter six, chemical characteristics that are important for CYP2E1 inhibition were evaluated.

Finally, the results of this thesis are discussed in chapter seven and summarized in chapter eight. 


\section{References}

1. Ludwig, J., et al., Nonalcoholic steatohepatitis. Mayo clinic experiences with a hitherto unnamed disease. Mayo Clinic Proc, 1980. 55: p. 434-438.

2. Chalasani, N., et al., The diagnosis and management of non-alcoholic fatty liver disease: practice guideline by the American Gastroenterological Association, American Association for the Study of Liver Diseases, and American College of Gastroenterology. Gastroenterology, 2012. 142(7): p. 1592-609.

3. Bedogni, G., et al., Prevalence of and risk factors for nonalcoholic fatty liver disease: the Dionysos nutrition and liver study. Hepatology, 2005. 42(1): p. 44-52.

4. Browning, J.D., et al., Prevalence of hepatic steatosis in an urban population in the United States: impact of ethnicity. Hepatology, 2004. 40(6): p. 1387-95.

5. Bjornsson, E. and P. Angulo, Non-alcoholic fatty liver disease. Scand J Gastroenterol, 2007. 42(9): p. 1023-30.

6. Williams, C.D., et al., Prevalence of nonalcoholic fatty liver disease and nonalcoholic steatohepatitis among a largely middle-aged population utilizing ultrasound and liver biopsy: a prospective study. Gastroenterology, 2011. 140(1): p. 124-31.

7. Smits, M.M., et al., Non-alcoholic fatty liver disease as an independent manifestation of the metabolic syndrome: results of a US national survey in three ethnic groups. J Gastroenterol Hepatol, 2013. 28(4): p. 664-70.

8. Lalor, P.F., et al., The role of cytokines and chemokines in the development of steatohepatitis. Semin Liver Dis, 2007. 27(2): p. 173-93.

9. Alberti, K.G., et al., Harmonizing the metabolic syndrome: a joint interim statement of the International Diabetes Federation Task Force on Epidemiology and Prevention; National Heart, Lung, and Blood Institute; American Heart Association; World Heart Federation; International Atherosclerosis Society; and International Association for the Study of Obesity. Circulation, 2009. 120(16): p. 1640-5.

10. Bellentani, S., et al., Epidemiology of non-alcoholic fatty liver disease. Dig Dis, 2010. 28(1): p. 15561.

11. Day, C.P. and O.F.W. James, Steatohepatitis: A Tale of Two "Hits"? Gastroenterology, 1998. 114(4): p. 842-845.

12. Tilg, H. and A.R. Moschen, Evolution of inflammation in nonalcoholic fatty liver disease: the multiple parallel hits hypothesis. Hepatology, 2010. 52(5): p. 1836-46.

13. Yilmaz, Y., Review article: is non-alcoholic fatty liver disease a spectrum, or are steatosis and nonalcoholic steatohepatitis distinct conditions? Alimentary pharmacology \& therapeutics, 2012. 36(9): p. 815-23.

14. Pais, R., et al., Progression from isolated steatosis to steatohepatitis and fibrosis in nonalcoholic fatty liver disease. Clin Res Hepatol Gastroenterol, 2011. 35(1): p. 23-8.

15. Harrison, S.A., S. Torgerson, and P.H. Hayashi, The natural history of nonalcoholic fatty liver disease: a clinical histopathological study. Am J Gastroenterol, 2003. 98(9): p. 2042-7.

16. Kim, S.R., et al., Two cases of non-alcoholic steato-hepatitis developing from simple fatty liver. J Gastrointestin Liver Dis, 2009. 18(4): p. 491-5.

17. Pais, R., et al., A systematic review of follow-up biopsies reveals disease progression in patients with non-alcoholic fatty liver. J Hepatol, 2013. 59(3): p. 550-6.

18. Wong, V.W., et al., Disease progression of non-alcoholic fatty liver disease: a prospective study with paired liver biopsies at 3 years. Gut, 2010. 59(7): p. 969-74.

19. Fabbrini, E., et al., Alterations in adipose tissue and hepatic lipid kinetics in obese men and women with nonalcoholic fatty liver disease. Gastroenterology, 2008. 134(2): p. 424-31.

20. Polyzos, S.A., et al., Nonalcoholic fatty liver disease: multimodal treatment options for a pathogenetically multiple-hit disease. Journal of clinical gastroenterology, 2012. 46(4): p. 272-84. 
21. Machado, M.V. and H. Cortez-Pinto, Non-alcoholic fatty liver disease: what the clinician needs to know. World J Gastroenterol, 2014. 20(36): p. 12956-80.

22. Farrell, G.C., et al., NASH is an Inflammatory Disorder: Pathogenic, Prognostic and Therapeutic Implications. Gut Liver, 2012. 6(2): p. 149-71.

23. Nguyen, T.A. and A.J. Sanyal, Pathophysiology guided treatment of nonalcoholic steatohepatitis. J Gastroenterol Hepatol, 2012. 27 Suppl 2: p. 58-64.

24. Ganz, M. and G. Szabo, Immune and inflammatory pathways in NASH. Hepatol Int, 2013. 7 Suppl 2: p. 771-81.

25. Kubes, P. and W.Z. Mehal, Sterile inflammation in the liver. Gastroenterology, 2012.143(5): p. 1158-72.

26. Szabo, G. and T. Csak, Inflammasomes in liver diseases. J Hepatol, 2012. 57(3): p. 642-54.

27. Tang, T., et al., Pro-inflammatory activated Kupffer cells by lipids induce hepatic NKT cells deficiency through activation-induced cell death. PLoS One, 2013. 8(12): p. e81949.

28. Bieghs, V., et al., Trapping of oxidized LDL in lysosomes of Kupffer cells is a trigger for hepatic inflammation. Liver Int, 2013. 33(7): p. 1056-61.

29. Zeng, T.S., et al., Depletion of Kupffer cells attenuates systemic insulin resistance, inflammation and improves liver autophagy in high-fat diet fed mice. Endocr J, 2015. 62(7): p. 615-26.

30. Lanthier, N., et al., Kupffer cell depletion prevents but has no therapeutic effect on metabolic and inflammatory changes induced by a high-fat diet. Faseb j, 2011. 25(12): p. 4301-11.

31. Zhang, X.Q., et al., Role of endoplasmic reticulum stress in the pathogenesis of nonalcoholic fatty liver disease. World J Gastroenterol, 2014. 20(7): p. 1768-76.

32. Koek, G.H., P.R. Liedorp, and A. Bast, The role of oxidative stress in non-alcoholic steatohepatitis. Clin Chim Acta, 2011. 412(15-16): p. 1297-1305.

33. Rolo, A.P., J.S. Teodoro, and C.M. Palmeira, Role of oxidative stress in the pathogenesis of nonalcoholic steatohepatitis. Free Radic Biol Med, 2012. 52: p. 59-69.

34. Ucar, F., et al., The relationship between oxidative stress and nonalcoholic fatty liver disease: Its effects on the development of nonalcoholic steatohepatitis. Redox report : communications in free radical research, 2013.18(4): p. 127-33.

35. Lieber, C.S., CYP2E1: from ASH to NASH. Hepatol Res, 2004. 28: p. 1-11.

36. Robertson, G., I. Leclercq, and G.C. Farrell, Nonalcoholic steatosis and steatohepatitis II. Cytochrome p-450 enzymes and oxidative stress. Am J Physiol Gastrointest Liver Physiol, 2001. 281: p. G1135-G1139.

37. Tariq, Z., C.J. Green, and L. Hodson, Are oxidative stress mechanisms the common denominator in the progression from hepatic steatosis towards non-alcoholic steatohepatitis (NASH)? Liver Int, 2014. 34(7): p. e180-90.

38. Pessayre, D., Role of mitochondria in non-alcoholic fatty liver disease. J Gastroenterol Hepatol, 2007. 22 Suppl 1: p. S20-7.

39. Tiniakos, D.G., M.B. Vos, and E.M. Brunt, Nonalcoholic fatty liver disease: pathology and pathogenesis. Annu Rev Pathol, 2010. 5: p. 145-71.

40. Videla, L.A., et al., Oxidative stress-related parameters in the liver of non-alcoholic fatty liver disease patients. Clin Sci (Lond), 2004. 106(3): p. 261-8.

41. Sreekumar, R., et al., Hepatic gene expression in histologically progressive nonalcoholic steatohepatitis. Hepatology, 2003. 38(1): p. 244-51.

42. Nelson, J.E., et al., Relationship between the pattern of hepatic iron deposition and histological severity in non alcoholic fatty liver disease. Hepatology, 2011. 53(2): p. 448-457.

43. Valenti, L., et al., HFE genotype, parenchymal iron accumulation, and liver fibrosis in patients with nonalcoholic fatty liver disease. Gastroenterology, 2010. 138(3): p. 905-912.

44. Fenton, H.J.H., Oxidation of tartaric acid in presence of iron. J Chem Soc Trans, 1894. 65: p. 899911.

45. Machado, M.V. and H. Cortez-Pinto, Gut microbiota and nonalcoholic fatty liver disease. Ann Hepatol, 2012. 11(4): p. 440-9. 
46. Minemura, M. and Y. Shimizu, Gut microbiota and liver diseases. World J Gastroenterol, 2015. 21(6): p. 1691-702.

47. Mouzaki, M., et al., Intestinal microbiota in patients with nonalcoholic fatty liver disease. Hepatology, 2013. 58(1): p. 120-7.

48. Rinella, M.E., Nonalcoholic fatty liver disease: a systematic review. Jama, 2015. 313(22): p. $2263-$ 73.

49. Abd El-Kader, S.M. and E.M. El-Den Ashmawy, Non-alcoholic fatty liver disease: The diagnosis and management. World J Hepatol, 2015. 7(6): p. 846-58.

50. Festi, D., et al., Review article: the diagnosis of non-alcoholic fatty liver disease -- availability and accuracy of non-invasive methods. Aliment Pharmacol Ther, 2013. 37(4): p. 392-400.

51. Machado, M.V. and H. Cortez-Pinto, Non-invasive diagnosis of non-alcoholic fatty liver disease. A critical appraisal. J Hepatol, 2013. 58(5): p. 1007-19.

52. Adams, L.A. and A.E. Feldstein, Nonalcoholic steatohepatitis: risk factors and diagnosis. Expert Rev Gastroenterol Hepatol, 2010. 4(5): p. 623-35.

53. Kleiner, D.E., et al., Design and validation of a histological scoring system for nonalcoholic fatty liver disease. Hepatology, 2005. 41(6): p. 1313-1321.

54. Ratziu, V., et al., Histological progression of non-alcoholic fatty liver disease: a critical reassessment based on liver sampling variability. Aliment Pharmacol Ther, 2007. 26(6): p. 821-30.

55. Fukusato, T., et al., Interobserver variation in the histopathological assessment of nonalcoholic steatohepatitis. Hepatol Res, 2005. 33(2): p. 122-7.

56. Kleiner, D.E., et al., Design and validation of a histological scoring system for nonalcoholic fatty liver disease. Hepatology, 2005. 41(6): p. 1313-21.

57. Strauss, S., et al., Interobserver and intraobserver variability in the sonographic assessment of fatty liver. AJR Am J Roentgenol, 2007. 189(6): p. W320-3.

58. Meisamy, S., et al., Quantification of hepatic steatosis with T1-independent, T2-corrected MR imaging with spectral modeling of fat: blinded comparison with MR spectroscopy. Radiology, 2011. 258(3): p. 767-75.

59. Federico, A., et al., Focus on emerging drugs for the treatment of patients with non-alcoholic fatty liver disease. World J Gastroenterol, 2014. 20(45): p. 16841-57.

60. Thoma, C., C.P. Day, and M.I. Trenell, Lifestyle interventions for the treatment of non-alcoholic fatty liver disease in adults: a systematic review. J Hepatol, 2012. 56(1): p. 255-66.

61. Musso, G., et al., Impact of current treatments on liver disease, glucose metabolism and cardiovascular risk in non-alcoholic fatty liver disease (NAFLD): a systematic review and metaanalysis of randomised trials. Diabetologia, 2012. 55(4): p. 885-904.

62. Vuppalanchi, R. and N. Chalasani, Nonalcoholic fatty liver disease and nonalcoholic steatohepatitis: Selected practical issues in their evaluation and management. Hepatology, 2009. 49(1): p. 306-17.

63. Harrison, S.A., et al., Orlistat for overweight subjects with nonalcoholic steatohepatitis: $A$ randomized, prospective trial. Hepatology, 2009. 49(1): p. 80-6.

64. Zelber-Sagi, S., et al., A double-blind randomized placebo-controlled trial of orlistat for the treatment of nonalcoholic fatty liver disease. Clin Gastroenterol Hepatol, 2006. 4(5): p. 639-44.

65. Mallat, A., F. Teixeira-Clerc, and S. Lotersztajn, Cannabinoid signaling and liver therapeutics. J Hepatol, 2013. 59(4): p. 891-6.

66. Van Gaal, L.F., et al., Long-term effect of CB1 blockade with rimonabant on cardiometabolic risk factors: two year results from the RIO-Europe Study. Eur Heart J, 2008. 29(14): p. 1761-71.

67. Despres, J.P., et al., Effect of rimonabant on the high-triglyceride/low-HDL-cholesterol dyslipidemia, intraabdominal adiposity, and liver fat: the ADAGIO-Lipids trial. Arterioscler Thromb Vasc Biol, 2009. 29(3): p. 416-23.

68. Li, Y., et al., Metformin in non-alcoholic fatty liver disease: A systematic review and meta-analysis. Biomed Rep, 2013. 1(1): p. 57-64.

69. Mazza, A., et al., The role of metformin in the management of NAFLD. Exp Diabetes Res, 2012. 2012: p. 716404. 
70. Boettcher, E., et al., Meta-analysis: pioglitazone improves liver histology and fibrosis in patients with non-alcoholic steatohepatitis. Aliment Pharmacol Ther, 2012. 35(1): p. 66-75.

71. Olaywi, M., et al., Novel anti-diabetic agents in non-alcoholic fatty liver disease: a mini-review. Hepatobiliary Pancreat Dis Int, 2013. 12(6): p. 584-8.

72. Ohki, T., et al., The effectiveness of liraglutide in nonalcoholic fatty liver disease patients with type 2 diabetes mellitus compared to sitagliptin and pioglitazone. ScientificWorldJournal, 2012. 2012: p. 496453.

73. Kenny, P.R., et al., Exenatide in the treatment of diabetic patients with non-alcoholic steatohepatitis: a case series. Am J Gastroenterol, 2010. 105(12): p. 2707-9.

74. Yilmaz, Y., et al., Effects of sitagliptin in diabetic patients with nonalcoholic steatohepatitis. Acta Gastroenterol Belg, 2012. 75(2): p. 240-4.

75. Iwasaki, T., et al., Sitagliptin as a novel treatment agent for non-alcoholic Fatty liver disease patients with type 2 diabetes mellitus. Hepatogastroenterology, 2011. 58(112): p. 2103-5.

76. Nseir, W., J. Mograbi, and M. Ghali, Lipid-lowering agents in nonalcoholic fatty liver disease and steatohepatitis: human studies. Dig Dis Sci, 2012. 57(7): p. 1773-81.

77. Eslami, L., et al., Statins for non-alcoholic fatty liver disease and non-alcoholic steatohepatitis. Cochrane Database Syst Rev, 2013. 12: p. Cd008623.

78. Scorletti, E. and C.D. Byrne, Omega-3 fatty acids, hepatic lipid metabolism, and nonalcoholic fatty liver disease. Annu Rev Nutr, 2013. 33: p. 231-48.

79. Parker, H.M., et al., Omega-3 supplementation and non-alcoholic fatty liver disease: a systematic review and meta-analysis. J Hepatol, 2012. 56(4): p. 944-51.

80. Sanyal, A.J., et al., No significant effects of ethyl-eicosapentanoic acid on histologic features of nonalcoholic steatohepatitis in a phase 2 trial. Gastroenterology, 2014. 147(2): p. 377-84.e1.

81. Dasarathy, S., et al., Double-blind randomized placebo-controlled clinical trial of omega 3 fatty acids for the treatment of diabetic patients with nonalcoholic steatohepatitis. J Clin Gastroenterol, 2015. 49(2): p. 137-44.

82. Scorletti, E., et al., Effects of purified eicosapentaenoic and docosahexaenoic acids in nonalcoholic fatty liver disease: results from the Welcome* study. Hepatology, 2014. 60(4): p. 1211-21.

83. Pathil, A., et al., Ursodeoxycholyl lysophosphatidylethanolamide improves steatosis and inflammation in murine models of nonalcoholic fatty liver disease. Hepatology, 2012. 55(5): p. 1369-78.

84. Xiang, Z., et al., The role of ursodeoxycholic acid in non-alcoholic steatohepatitis: a systematic review. BMC Gastroenterol, 2013. 13: p. 140.

85. Sanyal, A.J., et al., Pioglitazone, vitamin E, or placebo for nonalcoholic steatohepatitis. N Engl J Med, 2010. 362(18): p. 1675-85.

86. Miller, E.R., 3rd, et al., Meta-analysis: high-dosage vitamin E supplementation may increase allcause mortality. Ann Intern Med, 2005. 142(1): p. 37-46.

87. Bjelakovic, G., et al., Mortality in randomized trials of antioxidant supplements for primary and secondary prevention: systematic review and meta-analysis. Jama, 2007. 297(8): p. 842-57.

88. Berry, D., J.K. Wathen, and M. Newell, Bayesian model averaging in meta-analysis: vitamin $E$ supplementation and mortality. Clin Trials, 2009. 6(1): p. 28-41.

89. Gerss, J. and W. Kopcke, The questionable association of vitamin E supplementation and mortality-inconsistent results of different meta-analytic approaches. Cell Mol Biol (Noisy-le-grand), 2009. 55 Suppl: p. 0l1111-20.

90. Curtis, A.J., et al., Vitamin E supplementation and mortality in healthy people: a meta-analysis of randomised controlled trials. Cardiovasc Drugs Ther, 2014. 28(6): p. 563-73.

91. Imajo, K., et al., Microbiota and nonalcoholic steatohepatitis. Semin Immunopathol, 2014. 36(1): p. 115-32.

92. Qamar, A.A., Probiotics in Nonalcoholic Fatty Liver Disease, Nonalcoholic Steatohepatitis, and Cirrhosis. J Clin Gastroenterol, 2015. 49 Suppl 1: p. S28-32.

93. Ma, Y.Y., et al., Effects of probiotics on nonalcoholic fatty liver disease: a meta-analysis. World J Gastroenterol, 2013. 19(40): p. 6911-8. 
94. Kelishadi, R., S. Farajian, and M. Mirlohi, Probiotics as a novel treatment for non-alcoholic Fatty liver disease; a systematic review on the current evidences. Hepat Mon, 2013. 13(4): p. e7233.

95. Tarantino, G. and C. Finelli, Systematic review on intervention with prebiotics/probiotics in patients with obesity-related nonalcoholic fatty liver disease. Future Microbiol, 2015. 10(5): p. 889-902.

96. Lirussi, F., et al., Probiotics for non-alcoholic fatty liver disease and/or steatohepatitis. Cochrane Database Syst Rev, 2007(1): p. Cd005165.

97. Bower, G., et al., Bariatric Surgery and Non-Alcoholic Fatty Liver Disease: a Systematic Review of Liver Biochemistry and Histology. Obes Surg, 2015. 25(12): p. 2280-9.

98. Mummadi, R.R., et al., Effect of bariatric surgery on nonalcoholic fatty liver disease: systematic review and meta-analysis. Clin Gastroenterol Hepatol, 2008. 6(12): p. 1396-402.

99. Chavez-Tapia, N.C., et al., Bariatric surgery for non-alcoholic steatohepatitis in obese patients. Cochrane Database Syst Rev, 2010(1): p. Cd007340.

100. Wong, R.J., et al., Nonalcoholic steatohepatitis is the second leading etiology of liver disease among adults awaiting liver transplantation in the United States. Gastroenterology, 2015. 148(3): p. 54755.

101. Hassan, K., et al., Nonalcoholic fatty liver disease: a comprehensive review of a growing epidemic. World J Gastroenterol, 2014. 20(34): p. 12082-101.

102. Day, C.P., Natural history of NAFLD: remarkably benign in the absence of cirrhosis. Gastroenterology, 2005. 129(1): p. 375-8.

103. Matteoni, C.A., et al., Nonalcoholic fatty liver disease: a spectrum of clinical and pathological severity. Gastroenterology, 1999. 116(6): p. 1413-9.

104. Fassio, E., et al., Natural history of nonalcoholic steatohepatitis: a longitudinal study of repeat liver biopsies. Hepatology, 2004. 40(4): p. 820-6.

105. Bugianesi, E., et al., Expanding the natural history of nonalcoholic steatohepatitis: from cryptogenic cirrhosis to hepatocellular carcinoma. Gastroenterology, 2002. 123(1): p. 134-40.

106. Rafiq, N., et al., Long-term follow-up of patients with nonalcoholic fatty liver. Clin Gastroenterol Hepatol, 2009. 7(2): p. 234-8.

107. Adams, L.A., et al., The natural history of nonalcoholic fatty liver disease: a population based cohort study. Gastroenterology, 2005. 129(1): p. 113-121.

108. Ekstedt, M., et al., Long-term follow-up of patients with NAFLD and elevated liver enzymes. Hepatology, 2006. 44(4): p. 865-73.

109. Soderberg, C., et al., Decreased survival of subjects with elevated liver function tests during a 28 year follow-up. Hepatology, 2010. 51(2): p. 595-602. 



\section{Chapter 2}

\section{Elevated citrate levels in NAFLD: The potential of citrate to promote radical production}

Bregje van de Wier, Jiska M. Balk, Guido R.M.M. Haenen, Dimosthenis Giamouridis, Jaap. A. Bakker, Bertine C. Bast, Gertjan J.M. den Hartog, Ger H. Koek, Aalt Bast

FEBS letters. 2013; 587: 2461-2466 


\section{Abstract}

Plasma citrate levels were found to be elevated in non-alcoholic fatty liver disease (NAFLD) patients. Cellular experiments indicated that increased citrate levels might originate from an excess of fatty acids. The impact of elevated citrate levels on oxidative stress was examined. It was found that citrate stimulated hydrogen peroxide induced intracellular oxidative stress in HepG2 cells. This was related to the promotion of iron mediated hydroxyl radical formation from hydrogen peroxide by citrate. The stimulating effect of citrate on the reactivity of iron promotes oxidative stress, a crucial process in the progression of NAFLD. 


\section{Introduction}

Non-alcoholic fatty liver disease (NAFLD) is the most common liver disease in Western society with a prevalence of $20-30 \%[1,2]$. It is considered as the hepatic manifestation of the metabolic syndrome, which is caused by an unhealthy lifestyle including high caloric intake and low physical activity. NAFLD represents a spectrum of liver disorders ranging from steatosis to non-alcoholic steatohepatitis (NASH), fibrosis, cirrhosis and hepatocellular carcinoma. The majority of patients have benign steatosis only, but $25-30 \%$ of patients develop NASH [3], evolving to a systemic inflammation with the development of severe liver and cardiovascular diseases and cancer.

In the development of NASH, oxidative stress plays a crucial role. Oxidative stress is defined as an imbalance in the production of reactive oxygen species (ROS) and the protection against these ROS in favour of the production. Numerous causes of oxidative stress can be found in NAFLD [4]. Among others, oxidative processes in the mitochondria ( $\beta$-oxidation, citric acid cycle, oxidative phosphorylation) produce ROS, such as hydrogen peroxide and the superoxide radical. Several compounds, e.g. fatty acids, stimulate the generation of these ROS and thereby enhance oxidative stress [5].

In our outpatient clinic, patients presenting with liver test abnormalities were evaluated for NAFLD. During metabolic screening of the patients it was observed that citrate levels were elevated. The present study aims to elucidate the consequence of this observation and addresses the role of citrate in NAFLD. The focus is on the effect of citrate on oxidative stress.

\section{Materials and methods}

\section{Patients and tissue sampling}

Patients presenting with liver enzyme elevations were evaluated for NAFLD/NASH by extensive blood and urine investigations and liver biopsy. Patients who met the criteria for NAFLD/NASH according to liver biopsy were enrolled in this study. The study was performed following the ethical guidelines of the academic hospital and all patients gave informed consent to use their data. The data were collected according to routine clinical practice of our outpatient clinic and anonymised for the study. Active viral disease, alcoholic liver disease, haemochromatosis, copper storage disease, $\alpha-1$ - 
antitrypsine deficiency and drug induced liver disease were ruled out in all patients. Citrate blood plasma levels were determined in all patients included in the study.

\section{Reagents}

Minimum essential medium, fetal bovine serum, Dulbecco's phosphate buffered saline (DPBS), penicillin and streptomycin were purchased from Invitrogen (Breda, the Netherlands). Other reagents used were obtained from Sigma Aldrich (Zwijndrecht, the Netherlands).

\section{Cell culture}

Human hepatoma cells (HepG2 cells) were cultured at $37{ }^{\circ} \mathrm{C}$ under an atmosphere of $5 \% \mathrm{CO}_{2}$ in minimum essential medium containing $10 \%$ fetal bovine serum, $1 \%$ nonessential amino acids, $1 \mathrm{mM}$ sodium pyruvate, 100 units $/ \mathrm{ml}$ penicillin and $100 \mu \mathrm{g} / \mathrm{ml}$ streptomycin.

\section{Determination of citrate in HepG2 cells}

HepG2 cells were plated $5^{*} 10^{5}$ cells/well in a 6 wells plate. After growing to confluence, cells were exposed to serum free medium containing $1 \mathrm{mM}$ of palmitic acid and $1 \%$ bovine serum albumin for 24 hours. Control HepG2 cells were exposed to serum free medium containing $1 \%$ bovine serum albumin. After 24 hours, cells were harvested, counted and lysed. Citrate levels in the cell lysate were measured by liquid chromatography-tandem mass spectrometry, adapted from Keevil et al [6]. Cells were stained with Oil red 0 to confirm fat accumulation. For this purpose the cells were fixated with $10 \%$ formalin and washed with $60 \%$ isopropanol.

\section{Measurement of intracellular oxidative stress}

The production of intracellular reactive oxygen species was measured using 2,7dichlorofluorescin diacetate (DCFH-DA) [7]. HepG2 cells were plated $1 * 10^{5}$ cells/well in a 96 wells plate. After 24 hours, cells were incubated with $5 \mu \mathrm{M}$ DCFH-DA for 15 minutes at $37{ }^{\circ} \mathrm{C}$. After incubation with DCFH-DA, cells were washed with DPBS to remove any DCFH-DA that was not taken up by the cells. Then cells were exposed for one hour to $250 \mu \mathrm{M}$ sodium citrate or $500 \mu \mathrm{M}$ sodium citrate in DPBS or DPBS alone. 
After one hour, $500 \mu \mathrm{M}$ hydrogen peroxide $\left(\mathrm{H}_{2} \mathrm{O}_{2}\right)$ or DPBS was added and the fluorescence was measured (Ex 485, Em 538) for 30 minutes with the use of a Spectramax microplate reader.

\section{Cell viability measurement by lactate dehydrogenase leakage}

Cell viability was measured as leakage of lactate dehydrogenase (LDH) from cells in the medium. Directly after the incubation, the medium was collected and frozen. LDH activity was measured with use of the substrate pyruvate (initial concentration 100 $\mathrm{mM}$ ) as the reduction of the absorbance at $340 \mathrm{~nm}$ caused by the consumption of the cofactor NADH (initial concentration $0.35 \mathrm{mM}$ ). The rate of absorbance decline is proportional to LDH activity. LDH activity in the samples was compared to LDH activity in samples obtained of cells treated with Triton-X.

\section{Determination of hydroxyl radical production with deoxyribose}

Production of hydroxyl radicals $(\cdot \mathrm{OH})$ was measured with the use of 2-deoxy-D-ribose (deoxyribose) as detector [8]. In the reaction of deoxyribose with hydroxyl radicals, a breakdown product of deoxyribose is formed, that is detected after a condensation reaction with thiobarbituric acid which gives rise to an increase in absorption at 532 $\mathrm{nm}$. The reaction of ferrous ions $\left(\mathrm{Fe}^{2+}\right)$ with $\mathrm{H}_{2} \mathrm{O}_{2}$ generates hydroxyl radicals. This is known as the Fenton reaction [9]. The stimulating effect of citrate on the formation of hydroxyl radicals was determined. Reaction mixtures contained $2.8 \mathrm{mM}$ deoxyribose, $100 \mu \mathrm{M}$ ascorbic acid and varying concentrations of sodium citrate dissolved in a 20 $\mathrm{mM}$ potassium phosphate buffer ( $\mathrm{pH}$ 7.4). The reaction was started with the addition of $10 \mathrm{mM} \mathrm{H}_{2} \mathrm{O}_{2}$ and $100 \mu \mathrm{M}$ ferric chloride $\left(\mathrm{FeCl}_{3}\right)$. After one hour incubation at $37{ }^{\circ} \mathrm{C}$, $1.4 \%$ trichloroacetic acid and $0.5 \%$ thiobarbituric acid dissolved in milliQ water were added to stop the reaction and the mixtures were heated at $100{ }^{\circ} \mathrm{C}$ for 20 minutes. The absorption at $532 \mathrm{~nm}$ was measured with a Varian Cary 50 spectrophotometer.

\section{Determination of hydroxyl radical production with electron spin resonance}

To detect hydroxyl radicals produced in the Fenton reaction by electron spin resonance (ESR) using spin-trapping, $\mathrm{H}_{2} \mathrm{O}_{2}(10 \mathrm{mM})$ and ferrous sulphate $\left(\mathrm{FeSO}_{4}\right)$ $(100 \mu \mathrm{M})$ were incubated with or without citrate $(300 \mu \mathrm{M})$ in the presence of 5- 
diethoxyphosphoryl-5-methyl-1-pyrroline $\mathrm{N}$-oxide (DMPO) $(70 \mathrm{mM}$ ) in a $20 \mathrm{mM}$ potassium phosphate buffer. As a control the experiment was performed without $\mathrm{FeSO}_{4}$. All ESR experiments were performed at $9.8 \mathrm{GHz}$ using a Bruker ESR 300 EMX spectrometer (Bruker $\mathrm{GmbH}$, Karlsruhe, Germany) and operating at a centerfield strength of 3,488 $\mathrm{G}$ with $65 \mathrm{G}$ as sweep width, a modulation amplitude of $1 \mathrm{G}$ and microwave power of $10 \mathrm{~mW}$. The time constant and the conversion time were 40.96 and $20.48 \mathrm{~ms}$, respectively, and the results are the average of 10 scans. The ESR spin trapping experiments were performed by placing the samples in a $100 \mu \mathrm{l}$ glass capillary immediately after $\mathrm{H}_{2} \mathrm{O}_{2}$ addition, and the spectra were recorded within $1 \mathrm{~min}$ after starting the reaction. The height of the signal over the ESR spectrum is proportional to the amount of hydroxyl radicals formed.

\section{Iron (II) induced oxygen consumption}

The effect of citrate on oxygen consumption by a $100 \mu \mathrm{M} \mathrm{FeSO}{ }_{4}$ solution was measured using a Tamson YSI 5300 oxygen monitor. The $\mathrm{FeSO}_{4}$ solution was added to $1 \mathrm{ml} 50 \mathrm{mM}$ tris $\mathrm{HCl}$ buffer, with $150 \mathrm{mM} \mathrm{NaCl}\left(\mathrm{pH} 7.4\right.$ at $37^{\circ} \mathrm{C}$ ) while oxygen tension was measured [10]. The $\mathrm{FeSO}_{4}$ solution was prepared immediately before use. When indicated, $300 \mu \mathrm{M}$ of citrate was added before addition of the $\mathrm{FeSO}_{4}$ solution to investigate the effect of citrate on the oxygen consumption of $\mathrm{FeSO}_{4}$.

\section{Statistics}

Results were presented as mean \pm standard deviation. Statistics were performed using the student $\mathrm{t}$-test. Repeated measurements ANOVA was used for the time curves obtained during the intracellular oxidative stress measurements. A p-value below 0.05 was considered to be significant.

\section{Results}

\section{Citrate levels in NAFLD patients}

Eleven patients ( 5 male, 6 female) with a mean age of 55 years (range 38-70 years) were included in this study. Patients' characteristics are summarized in table 1 . All patients had one or more features of the metabolic syndrome according to ATP III criteria for clinical identification of the metabolic syndrome. They all were overweight $\left(\mathrm{BMI}>25 \mathrm{~kg} / \mathrm{m}^{2}\right)$ and 6 patients were obese (BMI $>30 \mathrm{~kg} / \mathrm{m}^{2}$ ) with mainly abdominal 
fat deposition. Nine patients had elevated blood pressure ( $>130 / 85 \mathrm{~mm} \mathrm{Hg}$ ), five patients had elevated triglyceride levels ( $\geq 1.7 \mathrm{mmol} / \mathrm{L})$ and nine patients had elevated fasting glucose levels ( $>5.6 \mathrm{mmol} / \mathrm{L}$ ). All patients did not take antihypertensive or lipid and glucose lowering drugs at time of the study. Liver biopsy confirmed NASH in 9 out of 11 patients. Liver biopsies in these patients showed signs of steatosis, lobular inflammation and in most patients fibrosis. Two patients had steatosis only. Citrate levels in NAFLD patients were significantly increased (mean 161 $\pm 35 \mu \mathrm{mol} / \mathrm{L}$ ) compared to reference values (mean $79 \pm 14 \mu \mathrm{mol} / \mathrm{L}),(\mathrm{p}<0.0001)$.

Table 1. Patient characteristics

\begin{tabular}{ll}
\hline Characteristics & Study population $(n=11)$ \\
\hline Age $(y r)$ & $55 \pm 11$ \\
Male gender $(n)$ & 5 \\
BMI $\left(\mathrm{kg} / \mathrm{m}^{2}\right)$ & $30.5 \pm 3.6$ \\
$\quad<25(n)$ & 0 \\
$\quad 25-29.9(n)$ & 5 \\
$\quad>30(n)$ & 6 \\
Hypertension $(>130 / 85)(n)$ & 9 \\
VGT $(U / L)$ & $161 \pm 125^{*}$ \\
AST $(U / L)$ & $59 \pm 22^{*}$ \\
ALT $(U / L)$ & $93 \pm 35^{*}$ \\
Fasting glucose $(\mathrm{mmol} / L)$ & $6.6 \pm 1.4^{*}$ \\
Total cholesterol $(\mathrm{mmol} / L)$ & $5.5 \pm 1.1$ \\
HDL cholesterol $(\mathrm{mmol} / L)$ & $1.3 \pm 0.3$ \\
LDL cholesterol $(\mathrm{mmol} / \mathrm{L})$ & $3.3 \pm 0.7$ \\
Triglyceride $(\mathrm{mmol} / \mathrm{L})$ & $1.9 \pm 0.9^{*}$ \\
Albumin $(\mathrm{g} / \mathrm{L})$ & $44.1 \pm 3.3$ \\
Citric acid plasma $(\mu \mathrm{mol} / \mathrm{L})$ & $161 \pm 35^{*}$ \\
\hline
\end{tabular}

* Above the reference values. Abbreviations: AST: aspartate aminotransferase, ALT: alanine aminotransferase, $\gamma \mathrm{GT}$ : gamma-glutamyl transpeptidase. Characteristics are presented as mean \pm standard deviation or as number of patients out of a total of eleven (n). 


\section{Stimulation of citrate formation in HepG2 cells by palmitic acid}

HepG2 cells treated with palmitic acid showed evident fat accumulation compared to untreated control cells (figure $1 \mathrm{~A}$ and $\mathrm{B}$ ). The intracellular citrate level was elevated in palmitic acid treated cells $(330.4 \pm 45.1 \mu \mathrm{mol} / \mathrm{L})$ compared to untreated control cells $(168.0 \pm 51.0 \mu \mathrm{mol} / \mathrm{L}),(\mathrm{p}<0.001)$ (figure $1 \mathrm{C})$.
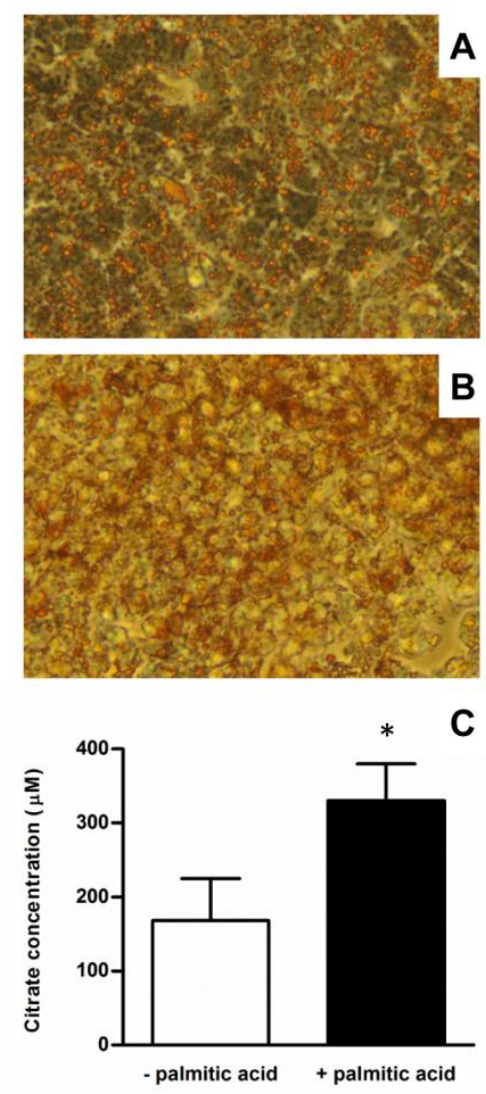

Figure 1. Effect of palmitic acid on intracellular citrate levels in HepG2 cells. A and B: Intracellular lipid accumulation in HepG2 cells treated with palmitic acid (B), compared to untreated HepG2 cells (A), shown by Oil red 0 staining, magnification 20 times. C: Intracellular citrate concentrations in HepG2 cells incubated with palmitic acid (+ palmitic acid) and in untreated HepG2 cells (- palmitic acid). Mean + SD are shown $(n=6) .{ }^{*} \mathrm{P}<0.001$. 


\section{Increase of intracellular oxidative stress by citrate}

HepG2 cells treated with 250 or $500 \mu \mathrm{M}$ citrate in combination with $\mathrm{H}_{2} \mathrm{O}_{2}$, showed a significant increase in the production of intracellular ROS compared to cells incubated with $\mathrm{H}_{2} \mathrm{O}_{2}$ alone (figure 2), $(\mathrm{p}<0.05)$. In control experiments, the formation of ROS in HepG2 cells treated with 250 and $500 \mu \mathrm{M}$ citrate did not increase compared to that in untreated cells (not depicted).

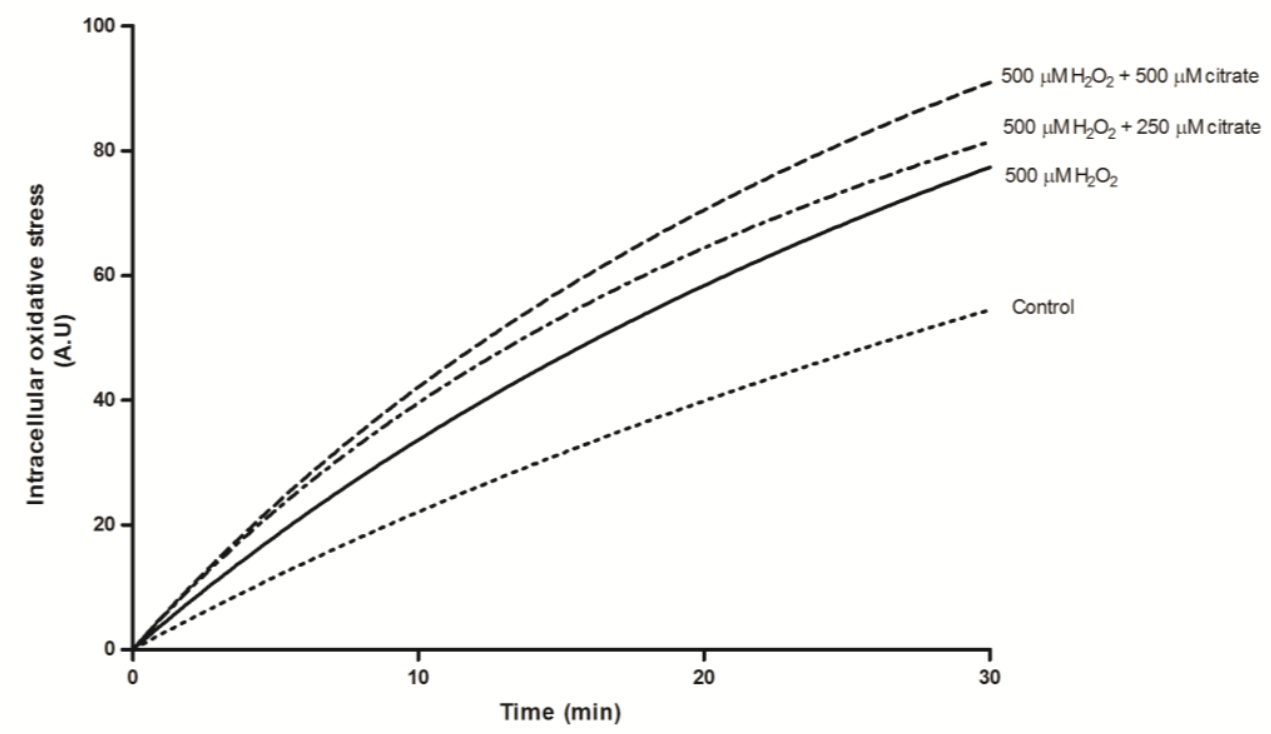

Figure 2. Stimulation of intracellular oxidative stress in HepG2 cells by citrate and $\mathrm{H}_{2} \mathrm{O}_{2}$. Intracellular oxidative stress in HepG2 cells incubated with 250 and $500 \mu \mathrm{M}$ citrate $+\mathrm{H}_{2} \mathrm{O}_{2}$, incubated with $\mathrm{H}_{2} \mathrm{O}_{2}$ alone and incubated in DPBS alone. Mean are shown ( $n=5)$. Repeated measurements ANOVA confirmed a significant difference in the increase in oxidative stress when using 250 and $500 \mu \mathrm{M}$ citrate $+\mathrm{H}_{2} \mathrm{O}_{2}$ compared to using $\mathrm{H}_{2} \mathrm{O}_{2}$ alone $(\mathrm{p}<0.05)$. Control experiments with citrate alone did not increase intracellular oxidative stress compared to the control (not depicted).

\section{Cell viability}

The viability of cells incubated in $500 \mu \mathrm{M} \mathrm{H}_{2} \mathrm{O}_{2}$ (mean $94.6 \pm 0.6 \%$ ), $500 \mu \mathrm{M}$ citrate (mean $90.0 \pm 3.5 \%$ ) or the combination of $\mathrm{H}_{2} \mathrm{O}_{2}$ and citrate (mean 88.6 $\pm 8.7 \%$ ) was not significantly different from the viability of cells incubated in PBS alone (mean 92.3 \pm 3.3 $\%)$. 
Stimulation of hydroxyl radical production by citrate determined with deoxyribose

Citrate increased the formation of hydroxyl radicals in the Fenton reaction (figure $3 \mathrm{~A}$ ) $(\mathrm{p}<0.05)$. A plateau in hydroxyl formation was reached at a concentration of $500 \mu \mathrm{M}$ of citrate.

\section{Stimulation of hydroxyl radical production by citrate determined with ESR}

The incubation of DMPO with $\mathrm{H}_{2} \mathrm{O}_{2}$ and ferrous ions resulted in DMPO/ $/ 0 \mathrm{OH}$ signal $\mathrm{z}(\mathrm{aN}$ and $\mathrm{aH} \beta=14.9 \mathrm{G})$ [11] (figure $3 \mathrm{~B})$. The addition of citrate $(300 \mu \mathrm{M})$ to the Fenton mixture increased the DMPO/ ${ }^{\circ} \mathrm{OH}$ signal (figure $3 \mathrm{~B}$ ), indicating an increase in the formation of hydroxyl radicals. The degree of increase was comparable to the increase found in the deoxyribose assay ( 1.3 times more ${ }^{\circ} \mathrm{OH}$ ). The combination of $\mathrm{H}_{2} \mathrm{O}_{2}$ and citrate without $\mathrm{Fe}^{2+}$ did not generate a DMPO/ $\cdot 0 \mathrm{OH}$ signal (not depicted). Also $\mathrm{H}_{2} \mathrm{O}_{2}$ alone did not result in a DMPO/•OH signal (not depicted). 


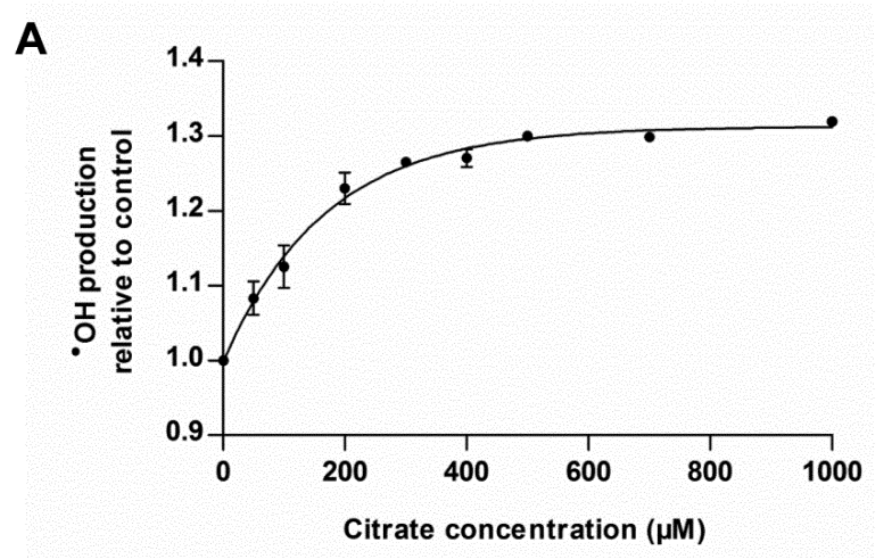

B
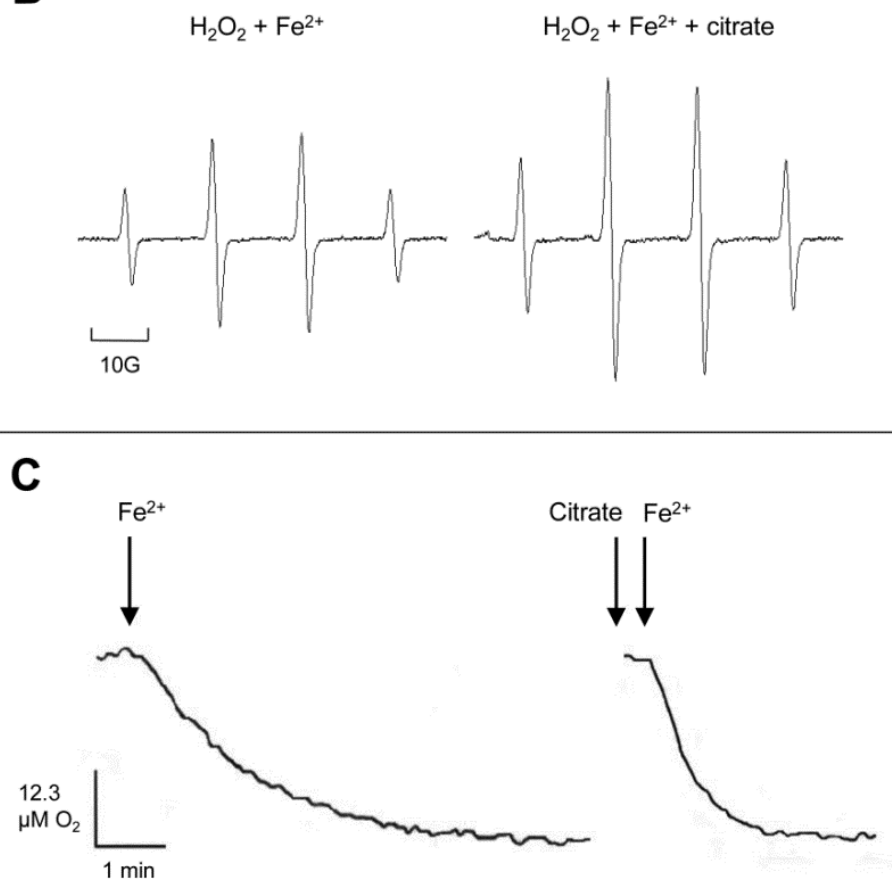

Figure 3. Stimulation of iron reactivity by citrate. A: Formation of hydroxyl radicals in the Fenton reaction measured with deoxyribose using various concentrations of citrate compared to control (no citrate). Mean \pm SD are shown $(n=2)$. B: Formation of hydroxyl radicals in the Fenton reaction with and without citrate measured with ESR. The hight of the ESR signal is proportional for the amount of hydroxyl radicals formed. C: Oxygen tension in a tris- $\mathrm{HCl}$ buffer after addition of a $\mathrm{FeSO}_{4}$ solution and after addition of a citrate solution and a $\mathrm{FeSO}_{4}$ solution, measured in an oxygen chamber. 


\section{Iron (II) induced oxygen consumption}

Addition of $100 \mu \mathrm{M}$ of $\mathrm{FeSO}_{4}$ to the tris- $\mathrm{HCl}$ buffer decreased oxygen tension with an initial rate of $6 \mu \mathrm{M} \mathrm{O}_{2} / \mathrm{min}$ (figure 3C). Citrate increased the oxygen consumption by $\mathrm{FeSO}_{4}$ threefold to a rate of $18 \mu \mathrm{M} \mathrm{O} / \mathrm{min}$ (figure 3C). Addition of citrate alone to the tris-HCl buffer did not decrease oxygen tension.

\section{Discussion}

The primary observation that initiated the present study was our finding that plasma citrate levels were increased in NAFLD patients $(101-210 \mu \mathrm{mol} / \mathrm{L})$ compared to reference values $(52-106 \mu \mathrm{mol} / \mathrm{L}),(\mathrm{p}<0.0001)$. The explanation for the elevated citrate levels can be found in the high levels of free fatty acids and glucose in these patients, associated with the metabolic syndrome. Both nutrients are broken down into acetyl-CoA, which leads to citrate formation in the citric acid cycle (figure 4). The citric acid cycle or Krebs cycle is the central metabolic process of the cell [12]. In the first step citrate is formed from oxaloacetate and acetyl-CoA. High levels of free fatty acids, seen in patients with the metabolic syndrome and NAFLD, boost the formation of citrate in the first step. This is caused by an increased acetyl-CoA influx into the citric acid cycle and a stimulation of oxaloacetate formation from pyruvate (figure 4). Because the third step in the citric acid cycle (isocitrate-dehydrogenase) is the key rate-limiting step and the second step in the cycle (aconitase) is reversible, citrate will accumulate. Experiments with HepG2 cells confirmed that high levels of fatty acids (palmitic acid) indeed lead to elevated citrate levels in the cytosol of liver cells.

From a chemical perspective, citrate is an odd intermediate in the oxidative breakdown of nutrients because it lacks a functional group that can be oxidized under physiological conditions; its three carboxyl groups are fully oxidized and its hydroxyl group is a tertiary alcohol that is resistant to oxidation. This chemical property and the accumulation in patients prompted us to further examine the influence of citrate on oxidative stress, as oxidative stress plays an important role in the progression of NAFLD to the chronic inflammatory status causing NASH.

Citrate added to HepG2 cells did not increase intracellular oxidative stress. However, oxidative stress did increase when citrate was used in combination with hydrogen peroxide. Hence, we conclude that citrate does not have an effect on oxidative stress directly, but citrate promotes oxidative stress indirectly. 


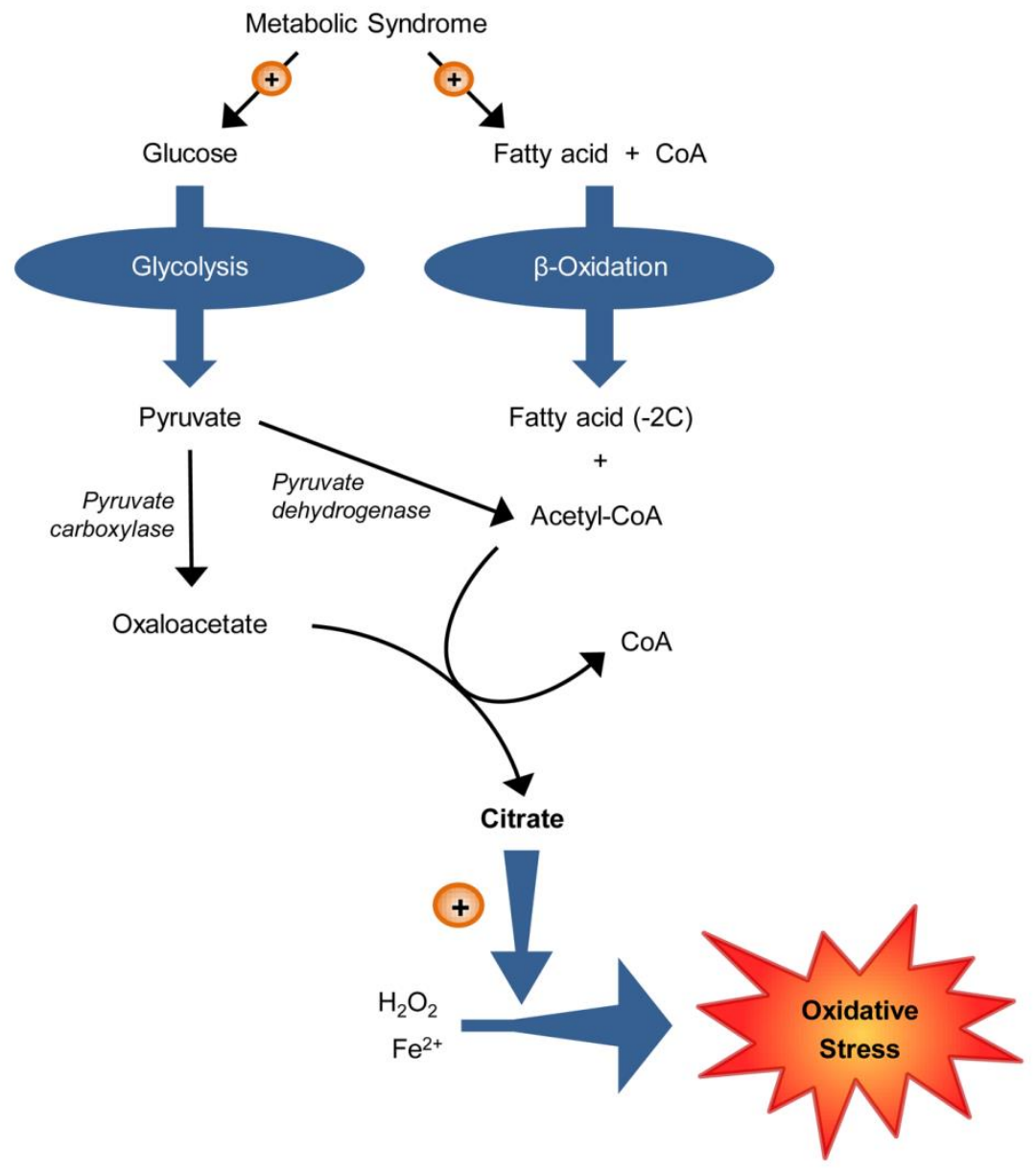

Figure 4. Schematic representation of the pathways leading to citrate formation. In the metabolic syndrome the presence of excessive fats and carbohydrates gives rise to elevated citrate levels according to the pathways depicted. During glycolysis glucose is converted into pyruvate. This is converted into oxaloacetate by pyruvate carboxylase and into acetyl-CoA by pyruvate dehydrogenase. During $\beta$-oxidation a fatty acid and coenzyme A (CoA) are converted into a two carbon atoms shorter fatty acid (fatty acid -2C) and acetylCoA. In the first step of the citric acid cycle, acetyl-CoA and oxaloacetate combine to form citrate. Citrate can promote oxidative stress by stimulating the reactivity of iron.

Apparently citrate aggravates oxidative stress caused by hydrogen peroxide. Hydrogen peroxide is not a radical by itself, because it does not contain an unpaired electron. It is relatively non-reactive. However, in combination with iron ions it leads to the formation of the highly reactive hydroxyl radical in the Fenton reaction [9]. The hydroxyl radical is considered to be the most noxious ROS, which can damage 
virtually any cellular component, including DNA, proteins and lipids. In experiments investigating the effect of citrate on the Fenton reaction (experiments with deoxyribose and ESR), citrate appeared to stimulate hydroxyl radical formation from hydrogen peroxide in the presence of iron. This is probably caused by its iron chelating ability. In the iron-citrate complex, iron apparently is more prone to react with hydrogen peroxide. Our findings corroborate previous studies that also demonstrated that citrate stimulates hydroxyl radical formation in the Fenton reaction [13-15]. Similar stimulating effects have been found for other chelating agents, such as EDTA $[13,14,16]$. In a study evaluating fourteen iron chelators, citrate appeared to be one of the strongest stimulators of iron-induced hydroxyl radical formation [13].

In the oxygen consumption experiment it was demonstrated that citrate also increases the reactivity of iron toward oxygen. Citrate enhances the rate of oxygen consumption by iron. This is in line with the effect of other iron chelating compounds that also increase iron reactivity toward oxygen [10].

In NAFLD patients, hepatic iron levels are commonly elevated $[17,18]$. Although the role of iron in NAFLD is multifactorial and not fully understood, the consensus is that this transition metal increases oxidative stress [19-21]. Promotion of radical production via the Fenton reaction is probably the most important cause of the increase in oxidative stress. The observed increase in oxidative stress in HepG2 cells treated with hydrogen peroxide and citrate could be explained by stimulation of iron reactivity, leading to an increase in hydroxyl radical formation. Serum ferritin, the iron storage protein, is also often elevated in patients with NAFLD. A correlation between serum ferritin levels and the severity of histologic abnormality has been observed in patients with NAFLD [22, 23]. Before iron stored in ferritin can promote radical formation, it has to be released from ferritin. Interestingly, the release of iron from ferritin requires chelation (e.g. by citrate) [24, 25]. Thus, apart from stimulating the production of hydroxyl radicals in the Fenton reaction directly, citrate could also increase oxidative stress in NAFLD by recruiting iron from ferritin, making more iron available for the Fenton reaction.

Apart from NAFLD, citrate levels are also elevated in citrin deficiency. Citrin deficiency is caused by a rare genetic defect that results in a dysfunctional mitochondrial aspartate-glutamate carrier. This leads to a compensatory up regulation of the malatecitrate carrier, resulting in elevated cytosolic citrate levels [26]. Patients with citrin deficiency can develop NAFLD and NASH without presenting other features of the 
metabolic syndrome. The development of NAFLD in these patients is still enigmatic, but as suggested by the data of the present study, the radical promoting potential of citrate might come into the picture in the pathogenesis of this disease

Although several treatments for NASH have been investigated, no registered drug therapy is available to date. The present study opens a new perspective for the treatment of NASH. The oxidative stress promoting effect of citrate might be counteracted by iron chelators that can compete with citrate for iron and that preferentially inhibit the Fenton reaction or scavenge the radicals that are formed.

In conclusion, the elevation of citrate levels measured in NAFLD patients can be caused by the breakdown of abundant free fatty acids. Citrate stimulates the reactivity of iron, which leads to promotion of radical formation. This results in an augmentation of oxidative stress which is of relevance for the progression of NAFLD into NASH. 


\section{References}

1. Lazo, M. and J.M. Clark, The epidemiology of nonalcoholic fatty liver disease: a global perspective. Semin Liver Dis, 2008. 28(4): p. 339-350.

2. Adams, L.A., et al., The natural history of nonalcoholic fatty liver disease: a population based cohort study. Gastroenterology, 2005. 129(1): p. 113-121.

3. McCullough, A.J., Pathophysiology of nonalcoholic steatohepatitis. J Clin Gastroenterol, 2006. 40(3 (Suppl 1)): p. S17-29.

4. Koek, G.H., P.R. Liedorp, and A. Bast, The role of oxidative stress in non-alcoholic steatohepatitis. Clin Chim Acta, 2011.412(15-16): p. 1297-1305.

5. $\quad$ Rolo, A.P., J.S. Teodoro, and C.M. Palmeira, Role of oxidative stress in the pathogenesis of nonalcoholic steatohepatitis. Free Radic Biol Med, 2012. 52: p. 59-69.

6. $\quad$ Keevill, B.G., et al., Measurement of citrate in urine using liquid chromatography tandem mass spectrometry: comparison with an enzymatic method. Ann Clin Biochem, 2005. 42(Pt5): p. 357 363.

7. Sohn, J., et al., Protective effects of panduratin A against oxidative damage of tertbutylhydroperoxide in human HepG2 cells. Biol Pharm Bull, 2005. 28(6): p. 1083-1086.

8. Halliwell, B., J.M.C. Gutteridge, and O.I. Aruoma, The deoxyribose method: A simple "test-tube" assay for determindation of rate constants for reactions of hydroxyl radicals. Anal Biochem, 1987. 165: $\mathrm{p}$. 215-219.

9. $\quad$ Fenton, H.J.H., Oxidation of tartaric acid in presence of iron. J Chem Soc Trans, 1894. 65: p. 899911.

10. Ursini, F., et al., Microsomal lipid peroxidation: mechanisms of initiation. The role of iron and iron chelators. Free Radic Biol Med, 1989. 6(1): p. 31-36.

11. Buettner, G.R., Spin trapping: ESR parameters of spin adducts. Free Radic Biol Med, 1987. 3(4): p. 259-303.

12. Krebs, H. and W.A. Johnson, The role of citric acid in intermediate metabolism in animal tissues. FEBS lett, 1980. 117(Suppl): p. K2-K10.

13. Gutteridge, J.M., Superoxide-dependent formation of hydroxyl radicals from ferric-complexes and hydrogen peroxide: an evaluation of fourteen iron chelators. Free radical research communications, 1990. 9(2): p. 119-25.

14. Engelmann, M.D., et al., Variability of the Fenton reaction characteristics of the EDTA, DTPA, and citrate complexes of iron. Biometals : an international journal on the role of metal ions in biology, biochemistry, and medicine, 2003. 16(4): p. 519-27.

15. Gautier-Luneau, I., et al., Iron-citrate complexes and free radicals generation: is citric acid an innocent additive in foods and drinks? Biometals : an international journal on the role of metal ions in biology, biochemistry, and medicine, 2007. 20(5): p. 793-6.

16. Grootveld, M. and B. Halliwell, An aromatic hydroxylation assay for hydroxyl radicals utilizing highperformance liquid chromatography (HPLC). Use to investigate the effect of EDTA on the Fenton reaction. Free Radic Res Commun, 1986. 1(4): p. 243-250.

17. Nelson, J.E., et al., Relationship between the pattern of hepatic iron deposition and histological severity in non alcoholic fatty liver disease. Hepatology, 2011. 53(2): p. 448-457.

18. Valenti, L., et al., HFE genotype, parenchymal iron accumulation, and liver fibrosis in patients with nonalcoholic fatty liver disease. Gastroenterology, 2010. 138(3): p. 905-912.

19. O'Brien, J. and L.P. Powell, Non-alcoholic fatty liver disease: Is iron relevant? Hepatol Int, 2011. 6(1): p. 332-341.

20. Dongiovanni, P., et al., Iron in fatty liver and in the metabolic syndrome: a promising therapeutic target. J Hepatology, 2011. 55(4): p. 920-932.

21. Corradini, E. and A. Pietrangelo, Iron and steatohepatitis. J Gastroenterol Hepatol, 2012. 27(Suppl 2): p. 42-46. 
22. Kowdley, K.V., et al., Serum ferritin is an independent predictor of histologic severity and advanced fibrosis in patients with nonalcoholic fatty liver disease. Hepatology, 2012. 55(1): p. 77-85.

23. Manousou, P., et al., Serum ferritin is a discriminant marker for both fibrosis and inflammation in histologically proven non-alcoholic fatty liver disease patients. Liver Int, 2011. 31(5): p. 730-739.

24. Reif, D.W., Ferritin as a source of iron for oxidative damage. Free Radic Biol Med, 1992. 12(5): p. 417-427.

25. Goddard, J.G., J.D. Gower, and C.J. Green, A chelator is required for microsomal lipid peroxidation following reductive ferritin-iron mobilisation. Free Radic Ress Commun, 1992. 17(3): p. 177-185.

26. Komatsu, M., et al., Citrin deficiency as a cause of chronic liver disorder mimicking non-alcoholic fatty liver disease. J Hepatol, 2008. 49: p. 810-820. 



\section{Chapter 3}

\section{The potential of flavonoids in the treatment of NAFLD}

Bregje van de Wier, Ger H. Koek, Aalt Bast, Guido R.M.M. Haenen

Critical Reviews in Food Science and Nutrition;

Accepted for publication 


\section{Abstract}

The contemporary pathophysiological model of non-alcoholic fatty liver disease (NAFLD) consists of multiple parallel pathways with a dynamic cross talk that cumulate in steatosis and inflammation and ultimately fibrosis, cirrhosis, liver failure and hepatocellular carcinoma. So far, no pharmacological treatment has been approved. A major impediment of drugs in general is that they are intended to act on one single target in the pathology of a disease. However, the multitude of pathways involved in the pathogenesis of NAFLD underpins the need for treatments that address these various pathways. Interestingly, flavonoids have been found to have positive effects on lipid metabolism, insulin resistance, inflammation and oxidative stress, the most important pathophysiological pathways in NAFLD. This puts flavonoids in the spotlight for the treatment of NAFLD and prompted us to review the existing evidence for the use of these food derived compounds in the treatment of NAFLD. 


\section{Introduction}

Non-alcoholic fatty liver disease (NAFLD) includes a spectrum of liver disorders, ranging from steatosis to non-alcoholic steatohepatitis (NASH), fibrosis, cirrhosis and hepatocellular carcinoma. The development of NAFLD is associated with the metabolic syndrome. Around 20 percent of the general population in Western countries has NAFLD [1] and 2 to 3 percent develops NASH [2]. The pathophysiological model that has evolved for the development of NAFLD includes multiple hits that do not follow a strict sequence (figure 1) [3]. In this model, metabolic disorders, oxidative stress and local and systemic inflammation are the major processes involved in the progression of NAFLD. These processes appear to enforce each other and a dynamic cross talk between the different processes exists (figure 1). Some authors suggest that steatosis and steatohepatitis might be two distinct disease entities [3, 4]. However, progression from steatosis to NASH has been documented in patients [5].

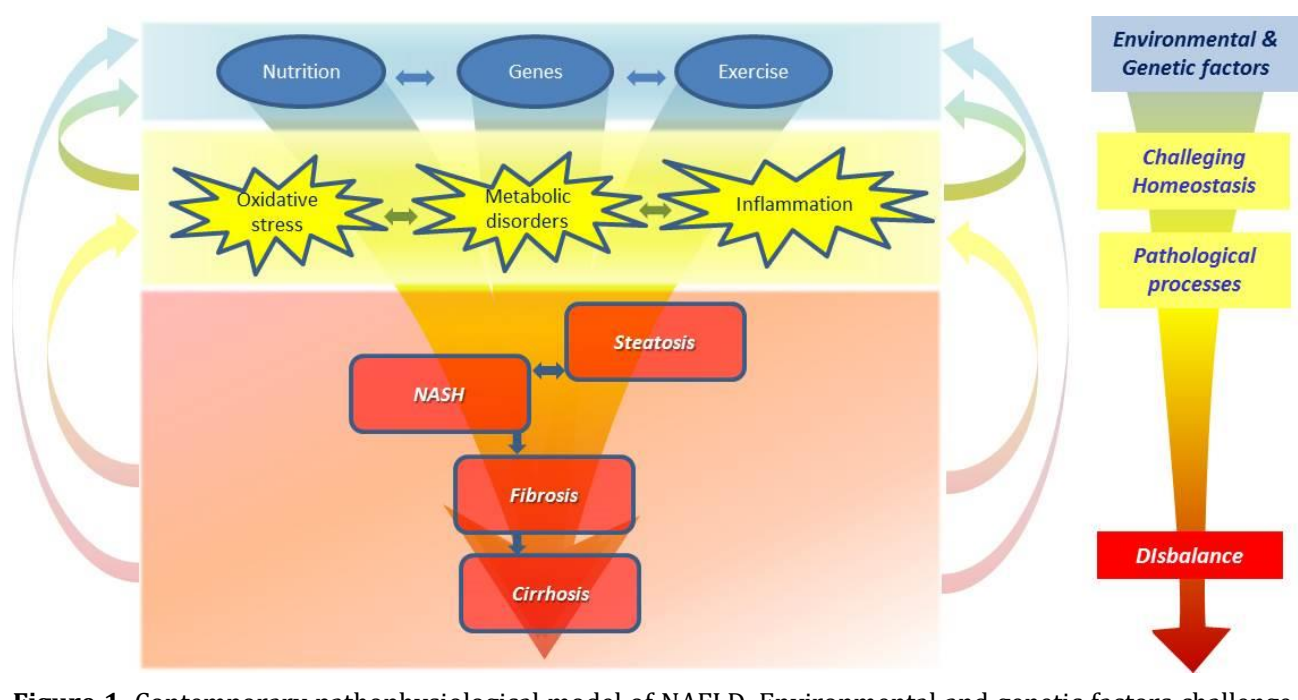

Figure 1. Contemporary pathophysiological model of NAFLD. Environmental and genetic factors challenge homeostasis and lead to several pathological processes: metabolic disorders, oxidative stress and inflammation. These processes also fortify each other and cause a disbalance, leading to the development of steatosis and non-alcoholic steatohepatitis (NASH). NASH can eventually progress to fibrosis and cirrhosis.

To date, no evidence based pharmacological treatment exists for NAFLD. Patients are given life style advice consisting of dietary recommendations as well as encouragement to increase physical exercise to lose weight. That a multitude of pathways has been implicated in the etiology of NAFLD makes the treatment challenging; ideally, the treatment should address all the multiple pathways. The 
involvement of multiple pathways explains why no effective drug has been found for the treatment of NAFLD. Traditionally, drugs are designed as "silver bullets" that, according to the classical medicinal chemical approach, have a well-defined, specific biologic target like a receptor (figure 2). Since such a well-defined, specific biologic target is missing in NAFLD, this traditional approach is deemed to fail.

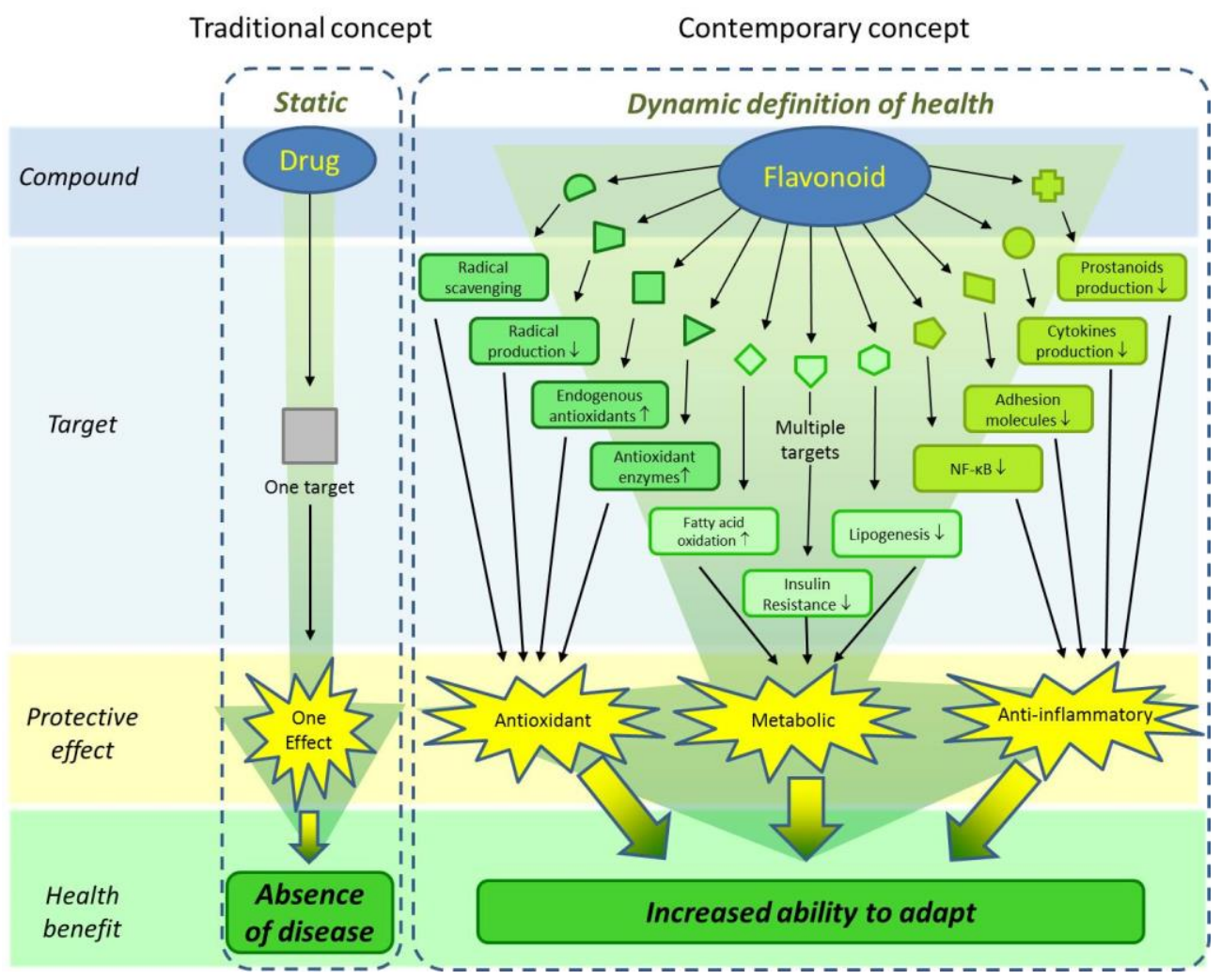

Figure 2. 'Traditional' concept of action of drugs versus the contemporary concept of action of bioactives, such as flavonoids. While traditional drugs are developed to act on one target, leading to absence of a disease, flavonoids act on multiple targets, affecting diverse pathological processes, leading to increased ability to adapt. This fits seamlessly in the pathophysiologic model of NAFLD, since diverse pathological processes are involved (figure 1).

Natural compounds, such as flavonoids, have frequently been studied in models of NAFLD and seem to display beneficial effects. Flavonoids comprise a diverse group of compounds abundantly found in our diet. The intake of flavonoids has been associated with several health benefits. Initially, their health benefits were attributed to their potent antioxidant activity. However, further research revealed that also other activities contribute, such as anti-inflammatory and metabolic effects. 
Apparently, flavonoids display a multitude of activities and therefore these compounds were upgraded from antioxidant to bioactive [6]. A bioactive produces a biological response via an array of subtle effects via different targets (figure 2). This multifarious mode of action of flavonoids seems to suit seamlessly in the treatment of NAFLD, in which various pathways are involved. This concept will be reviewed in the present paper.

Firstly, an inventory of the various pathways identified in the etiology of NAFLD will be made. Secondly, the several biological activities of flavonoids will be presented. These activities will be linked to the pathways involved in NAFLD explaining the rational for the use of flavonoids in the treatment of NAFLD. Finally, the clinical studies on the efficacy of flavonoids in NAFLD will be evaluated, with the focus on the effect of flavonoids on the different pathways.

To this end, a literature search was conducted on PubMed in November 2013 using the search terms: flavonoids, non-alcoholic fatty liver disease and non-alcoholic steatohepatitis. To increase the number of studies found, a search using the name of specific flavonoids was also included.

\section{Pathogenesis of NAFLD}

The pathogenesis of NAFLD is complex and multifactorial, comprising multiple hits that lead to steatosis and NASH $[3,7]$. Although various pathways have been identified, the list is not complete as the etiology still is enigmatic and our knowledge of the disease is progressing.

A hallmark in the development of NAFLD is the accumulation of fat in the liver. Factors that are known to contribute to this accumulation include: (1) high free fatty acids (FFA) supply due to increased lipolysis from visceral and subcutaneous adipose tissue and dietary intake, (2) low FFA oxidation in relation to the supply of FFA, (3) high hepatic lipogenesis and (4) low hepatic excretion of very low density lipoprotein (VLDL) (figure 3) [3]. Another hallmark is chronic inflammation causing fibrosis. Underlying processes include oxidative stress and lipid peroxidation, mitochondrial dysfunction, adipocytokine/cytokine imbalance, gut-derived bacterial endotoxins, hepatic stellate cell activation and genetic factors [7]. Also activation of Kupffer cells by cholesterol crystals is suggested to be a trigger for hepatic inflammation [8]. Insulin resistance plays an important role in the development of both steatosis and 
inflammation [7]. Due to insulin resistance, lipolysis is not inhibited by insulin. The FFA's released cause inflammation, promote ectopic fat deposition and further enhance insulin resistance, creating a self-propelling feed forward process (figure 3) [7]. Furthermore, insulin resistance stimulates gluconeogenesis in hepatocytes and reduces glycogen formation. Increased glucose and insulin levels stimulate de novo lipogenesis via hepatic transcription factors such as sterol regulatory element binding protein-1c (SREBP-1c) and carbohydrate response element binding protein (ChREBP). This causes stimulation of lipogenic enzymes such as glucokinase (gk), fatty acid synthase (FAS) and acetyl-coenzyme A carboxylase (ACC) (figure 3) [7].

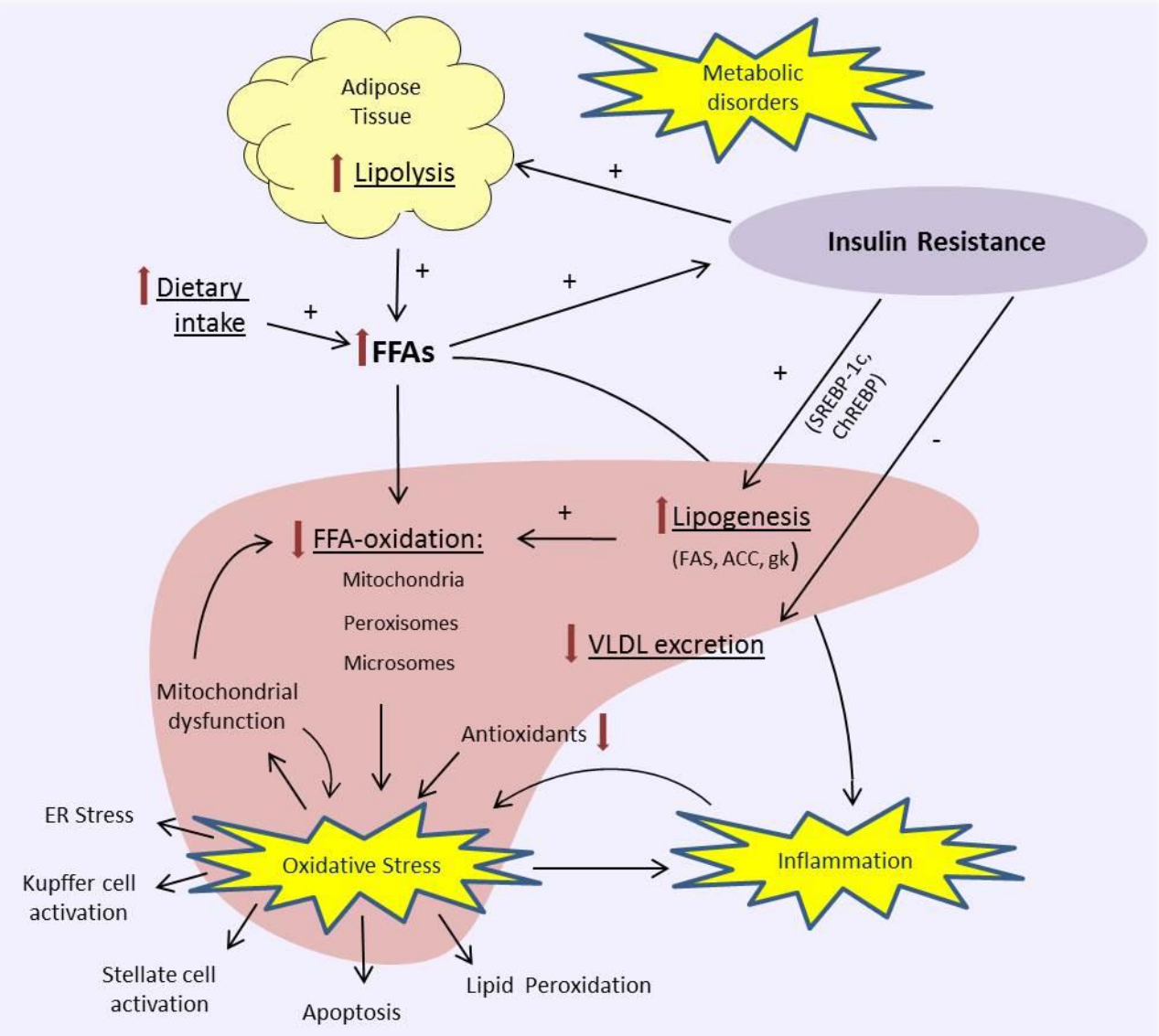

Figure 3. Schematic view of the pathophysiological pathways involved in NAFLD. Overview of the processes in liver, adipose and other tissues that are responsible for the metabolic disturbances, oxidative stress and inflammation in NAFLD. Abbreviations: FFA = free fatty acids, SREBP-1c = sterol regulatory elementbinding protein 1c, ChREBP = carbohydrate-responsive element-binding protein, FAS = fatty acid synthase, $\mathrm{ACC}=$ acetyl-CoA carboxylase, $\mathrm{gk}=$ glucokinase, $\mathrm{VLDL}=$ very low-density lipoprotein, $\mathrm{ER}=$ endoplasmic reticulum. 
In the multifactorial etiology of NASH, oxidative stress represents a crucial process [911]: the production of reactive oxygen species (ROS) not balanced by the protection against ROS by antioxidants. Various potential sources of oxidative stress have been reported in NAFLD. ROS are produced during the mitochondrial and peroxisomal beta oxidation of FFAs and during the metabolism of FFAs by cytochrome P450 2E1 and 4A. ROS cause endoplasmic reticulum (ER) stress, which further promotes the accumulation of ROS within the cell [11]. Also reduction in antioxidant defences will contribute to oxidative stress. Reduced glutathione (GSH) levels and decreased superoxide dismutase (SOD), glutathione peroxidase (GPx), catalase and glutathione transferase activities are found in NASH and appear to be correlated to disease severity [10]. ROS react with biological compounds including fatty acids, proteins and DNA, causing lipid peroxidation, mitochondrial dysfunction, stellate cell activation, inflammation (via NF-KB activation) and apoptosis (figure 3) $[9,11]$. Mitochondrial dysfunction and inflammation will lead to the formation of more ROS, further fuelling the self-propelling feed forward process.

Iron has also been implemented in the pathogenesis of NAFLD. In patients with NAFLD elevated hepatic iron levels have been found $[12,13]$. The precise role of iron in the pathogenesis of NAFLD has not yet been established, but it is well documented that iron increases oxidative stress, e.g. by its ability to generate hydroxyl radicals via the Fenton reaction [14]. Citrate, an intermediate product of lipid metabolism found to be elevated in NAFLD patients, has the ability to further increase this iron-induced oxidative stress by stimulation of the Fenton reaction [15].

\section{Flavonoids}

Flavonoids are polyphenolic compounds that are ubiquitously found in nature. They are secondary metabolites in plants and are frequently bound to sugars (glycosides) [16]. Flavonoids also occur as aglycones (without a sugar group) [16]. Most flavonoids (figure 4) consist of three rings: two aromatic rings (A and B) and one heterocyclic ring (C). Flavonoids are categorized into subclasses based on variations in the $\mathrm{C}$ ring. The major subclasses are flavones, isoflavones, flavanols, flavanones, anthocyanidins and chalcones (figure 4) [16].

Over 5,000 different flavonoids have been identified. Because the group of flavonoids is a very heterogenic group of compounds that act on multiple biological targets, various, sometimes even paradoxical, effects have been described for different classes 
of flavonoids. Also, the wide variation in models used to study the effects of flavonoids contributes to the variation in the effects found. This review addresses the potential of flavonoids and focusses on the reported positive effects of these bioactives in the treatment of NAFLD. These effects are differentiated in metabolic, antioxidant and anti-inflammatory effects.

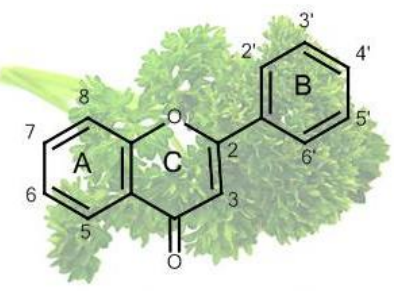

Flavones

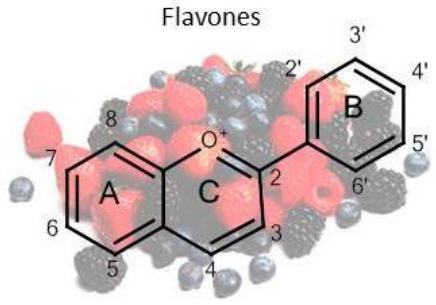

Anthocyanidins

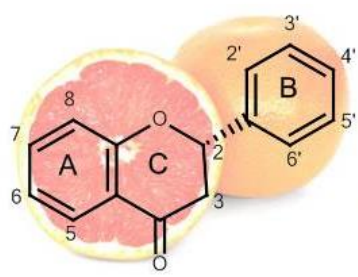

Flavanones

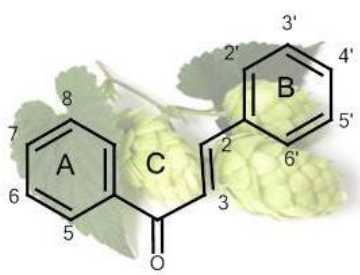

Chalcones

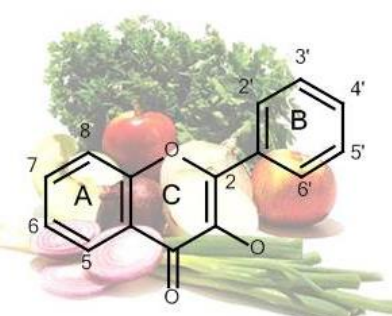

Flavonols

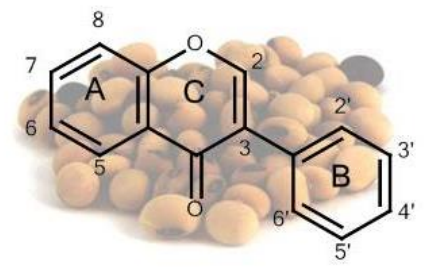

Isoflavones

Figure 4. Major subclasses of flavonoids found in significant amounts in the pictured fruits and vegetables.

\section{Metabolic effects}

\section{PPARs}

The peroxisome proliferator activated receptors (PPARs) are promising targets in the treatment of NAFLD. PPARs are nuclear receptors that play a role in the regulation of lipid and glucose metabolism as well as inflammation [17]. PPAR $\alpha$ is highly expressed in the liver and regulates FFA transport and stimulates enzymes involved in $\beta$ oxidation [18]. Furthermore, it limits inflammation by inhibition of NF- $\kappa B$ and reduction of C-reactive protein expression [17]. Studies have found evidences for a role of PPAR $\alpha$ in NAFLD and its treatment $[18,19]$. PPAR $\alpha /$ mice have increased susceptibility for development of NAFLD when fed a high fat diet compared to wildtype mice [18]. In addition, PPAR $\alpha$ agonists, such as fibrates, reduce steatosis, inflammation and fibrosis in models of NASH $[17,20]$. Stimulation of PPAR $\alpha$ is expected to decrease steatosis by stimulation of $\beta$-oxidation and to mitigate inflammation by inhibition of NF- KB. Several studies have demonstrated that 
flavonoids stimulate PPAR $\alpha$ [21-27]. For this stimulation various mechanisms of action have been proposed. Some studies claim that flavonoids are ligands and (partial) agonists of PPAR $\alpha[24,26,27]$. Other studies conclude that flavonoids upregulate PPAR $\alpha$ gene and/or protein expression [21-23, 25], possibly involving adiponectin, a stimulator of PPAR $\alpha$ [23]. As stated above, the variation in reported mechanisms might originate from the variation in chemical structure between flavonoids and the multitude of targets the flavonoids act on.

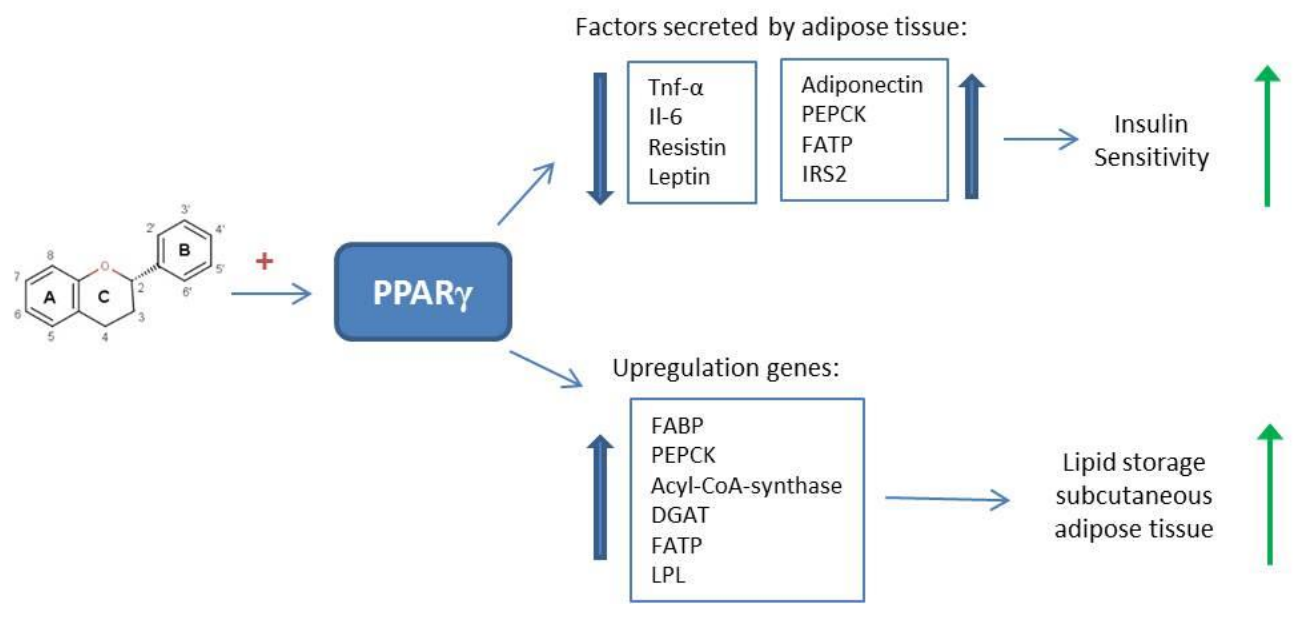

Figure 5. Effects of PPAR $\gamma$ stimulation by flavonoids. PPAR $\gamma$ stimulation by flavonoids (F) leads to changes in the factors secreted by adipose tissue, stimulating insulin sensitivity. Furthermore, it leads to the upregulation of specific genes, stimulating lipid storage in subcutaneous adipose tissue instead of the liver. Abbreviations: Tnf- $\alpha=$ tumor necrosis factor $\alpha$, Il- $6=$ interleukin 6 , PEPCK $=$ phospoenolpyruvate carboxykinase, FATP = fatty acid transport protein, IRS2 = insulin receptor substrate 2, FABP = fatty acidbinding protein, $\mathrm{ACS}=$ acetyl-CoA synthase, $\mathrm{DGAT}=$ diglyceride acyltransferase, $\mathrm{LPL}=$ lipoprotein lipase

PPAR $\gamma$ is mainly expressed in adipose tissue and its activation results in adipocyte differentiation and insulin sensitization [27]. Secretion of insulin resistance promoting factors by adipose tissue is reduced and secretion of insulin sensitivity promoting factors is increased (figure 5). By upregulation of adiponectin, PPAR $\gamma$ also activates PPAR $\alpha$. Furthermore, by activation of the involved genes (FABP, PEPCK, Acyl-CoA synthase, DGAT, FATP and LPL) adipogenesis and lipid storage in subcutaneous adipose tissue is stimulated (figure 5). Consequently, fat from harmful visceral adipose tissue is redistributed to subcutaneous fat depots [27]. Moreover, FFA delivery to the liver is reduced $[17,27]$. PPAR $\gamma$ has also been reported to increase energy expenditure by induction of uncoupling protein-2 (UCP-2) [18]. Mutations in the PPAR $\gamma$ gene increase the risk of developing metabolic syndrome and NAFLD [28]. 
Additionally, glitazones, PPAR $\gamma$ agonists, improve insulin resistance and decrease aminotransferase levels and liver fat in NASH patients, whereas positive effects on histological markers of NASH are not always noted [17]. These findings substantiate the use of PPAR $\gamma$ agonists in the treatment of NASH. Several studies report that flavonoids stimulate PPAR $\gamma$ [27, 29-33]. Similar as for PPAR $\alpha$ stimulation, various mechanisms of action are described. Flavonoids have been found to upregulate PPAR $\gamma$ gene expression [30,31,33,34]. Also, some flavonoids were observed to be agonists of PPAR $\gamma$ [27, 29]. An advantage of flavonoids over other drugs, like the glitazones, could be that the bioactives only partially activate PPAR $\gamma$. This reduces the risk of serious side effects seen with the use of full agonists. For example, weight gain, an important side effect of glitazones, is not associated with intake of isoflavones that also activate PPARy. In several studies, intake of isoflavones even leads to a slight weight reduction [27].

\section{SREBP-1C}

Another target identified in the treatment of NAFLD is SREBP-1c. SREBP-1c is a transcription factor that controls de novo lipogenesis via induction of the lipogenic enzymes (FAS, ACC, gk) [35], which stimulates steatosis. In liver biopsies of NAFLD patients, the expression of SREBP-1c and liver X receptor $\alpha$ (LXR $\alpha$ ), which controls SREBP-1c transcription, as well as the expression of ACC and FAS are found to be significantly higher than in control biopsies [36]. Several flavonoids inhibit SREBP-1c [34, 37-41]. Multiple mechanisms of SREBP-1c inhibition have been implicated. Various studies have found that flavonoids downregulate SREBP-1c protein and gene expression [34, 37-39, 41]. The isoflavone genistein reduces the expression of site-1 proteases, which are necessary for SREBP-1c to act as a transcription factor [40]. Furthermore, SREBP-1c is inhibited by activation of 5 ' adenosine monophosphateactivated protein kinase (AMPK) [38, 39, 41]. Activation of AMPK inhibits LXR $\alpha$, which controls SREBP-1c transcription [42]. Flavonoids stimulate activation of AMPK [25, 38, 39, 41]. Additionally, flavonoids inhibit LXR $\alpha$ [34, 37, 43]. Since SREBP-1c transcription is stimulated by hyperinsulinemia, flavonoids might also reduce SREBP1c expression by improving insulin sensitivity and normalizing insulin levels. Recently, activation of SREBP-1c was suggested to be one of the consequences of ER stress in the steatotic liver [35]. Inhibition of ER stress is another way in which flavonoids might inhibit SREBP-1c. 


\section{Antioxidant effects}

As a first line of defence, flavonoids reduce the production of radicals and other reactive species. Flavonoids inhibit pro-oxidant enzymes, such as xanthine oxidase [44]. Also inhibition of lipoxygenases and cyclooxygenases (see anti-inflammatory effects), enzymes that are capable to co-oxidize molecules other than their usual substrates, reduces the production of reactive species [44].

Flavonoids have been found to be very effective scavengers. This is a pivotal biochemical mode of action of bioactives, although the importance of radical scavenging is exaggerated as well as undervalued [6]. Amongst antioxidants, flavonoids are at the top of the pecking order, meaning that they are the first in line to scavenge radicals. They scavenge a wide array of reactive species including superoxide, hydroxyl, peroxyl and peroxynitrite radicals [45-47]. During this scavenging, flavonoids are oxidized by the radical, resulting in a more stable, less reactive radical [44]. Among the various subclasses of flavonoids, the flavonols that comprises quercetin and related flavonoids display superior scavenging activity. This is due to a large conjugated $\pi$-system that delocalizes electrons over the entire molecule. Structure-activity relationship studies reveal that two pharmacophores are present in the flavonols, (1) a catechol moiety in ring B and (2) a hydroxyl (OH) group at the 3 position at a 2,3-double bound, which is activated by the hydroxyl groups at the 5 and 7 position [48]. In NAFLD, radicals produced during peroxisomal and mitochondrial $\beta$-oxidation and the metabolism of FFA by Cytochrome P450 2E1 and $4 \mathrm{~A}$ can be scavenged by flavonoids, which will result in a reduction of oxidative stress.

In addition to the direct radical scavenging effect, several flavonoids have the ability to chelate iron and other transition metals that contribute to the formation of radicals $[16,49]$. The quercetin derived semi-synthetic flavonoid monoHER can scavenge $\mathrm{OH}-$ radicals at an extremely high apparent rate - ks $=980 \times 10^{8} \mathrm{M}^{-1} \mathrm{~s}^{-1}$ - which is even quicker than the diffusion rate $\left(\sim 100 \times 10^{8} \mathrm{M}^{-1} \mathrm{~s}^{-1}\right)$ [50]. This can be explained by 'site-specific scavenging'. Essential for this activity is that monoHER can chelate iron. The result of this chelation is that monoHER is present at the site of the radical formation, i.e. the iron ion. This enables monoHER to immediately scavenge the newly formed radical. By this site-specific scavenging, monoHER is able to prevent damage to critical biomolecules such as lipids, proteins or DNA, despite the high reactivity of the radical $[50,51]$. Since iron mediated hydroxyl radical formation has been implied 
in NAFLD [15, 52], this action of flavonoids is expected to be meaningful for the treatment of NAFLD.

A third mode of action is that flavonoids can protect or enhance the endogenous antioxidant defence [16, 53]. Several flavonoids induce glutathione S-transferase, heme-oxygenase 1 (HO-1) and other antioxidants [16, 54-57]. An important pathway in this response is stimulation of nuclear factor erythroid derived 2 (NRF2), a transcription factor that binds to antioxidant response elements (AREs) in the promoter region of genes encoding various antioxidants and phase II detoxifying enzymes. This leads to the transcription of those enzymes, e.g. HO-1 and NAD(P)Hquinone oxidoreductase [58]. Stimulation of NRF2 by flavonoids is reported in several studies [54-57]. Flavonoids increase NRF2 nuclear translocation to the nucleus and the binding of nrf2 to AREs [55,57]. Since depletion of antioxidant defences is seen in NASH patients, upregulation of antioxidants could be beneficial in the treatment of NASH [10].

\section{Anti-inflammatory effects}

Anti-inflammatory effects of flavonoids have been mainly subscribed to inhibition of the NF- $\kappa$ B pathway [59]. The NF- $\kappa B$ pathway consists of a canonical and noncanonical pathway. The canonical pathway is involved in inflammatory responses, while the non-canonical pathway regulates immune cell differentiation and maturation and lymphoid organogenesis [60]. Since the canonical pathway is most important for initiation of inflammation and effects of flavonoids focus on this part of the NF-KB pathway, our review is restricted to the canonical pathway. The canonical pathway is activated by pro-inflammatory signals, such as cytokines and oxidative stress [61]. This causes the IKK complex to phosphorylate and designate the IKBs $(\alpha, \beta$

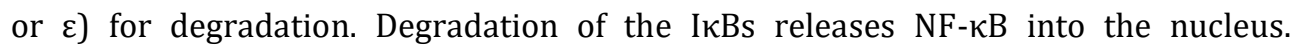
Consequently, transcriptional activity occurs resulting in an inflammatory response [60].

NF- $\kappa \mathrm{B}$ activation occurs in various inflammatory diseases. Activation of the NF- $\mathrm{\kappa B}$ pathway is observed in animal models of NASH as well as in NASH patients [62], illustrating a role for NF- $\kappa B$ activation in NASH. Flavonoids interfere with the NF- $\kappa B$ pathway in several ways (figure 6) [59]. Flavonoids inhibit the IKK complex and IкBphosphorylation, thereby preventing NF- $\mathrm{KB}$ translocation to the nucleus and the transcription of genes involved in the inflammatory response. Flavonoids also inhibit protein kinases that control the activity of NF-kB [63]. In addition, flavonoids inhibit 
protein kinase $\mathrm{C}(\mathrm{PKC})$, mitogen activated protein kinases (MAPKs), extracellular signal related kinase (ERK) and Jun N-terminal kinase (JNK) [63]. A mechanism proposed for inhibition of protein kinases is competitive binding to nucleotide binding sites [64]. Since oxidative stress also results in NF- $\kappa B$ activation [61], the antioxidant potency of flavonoids is also implicated in inhibition of the NF- $\kappa B$ pathway [65-67].

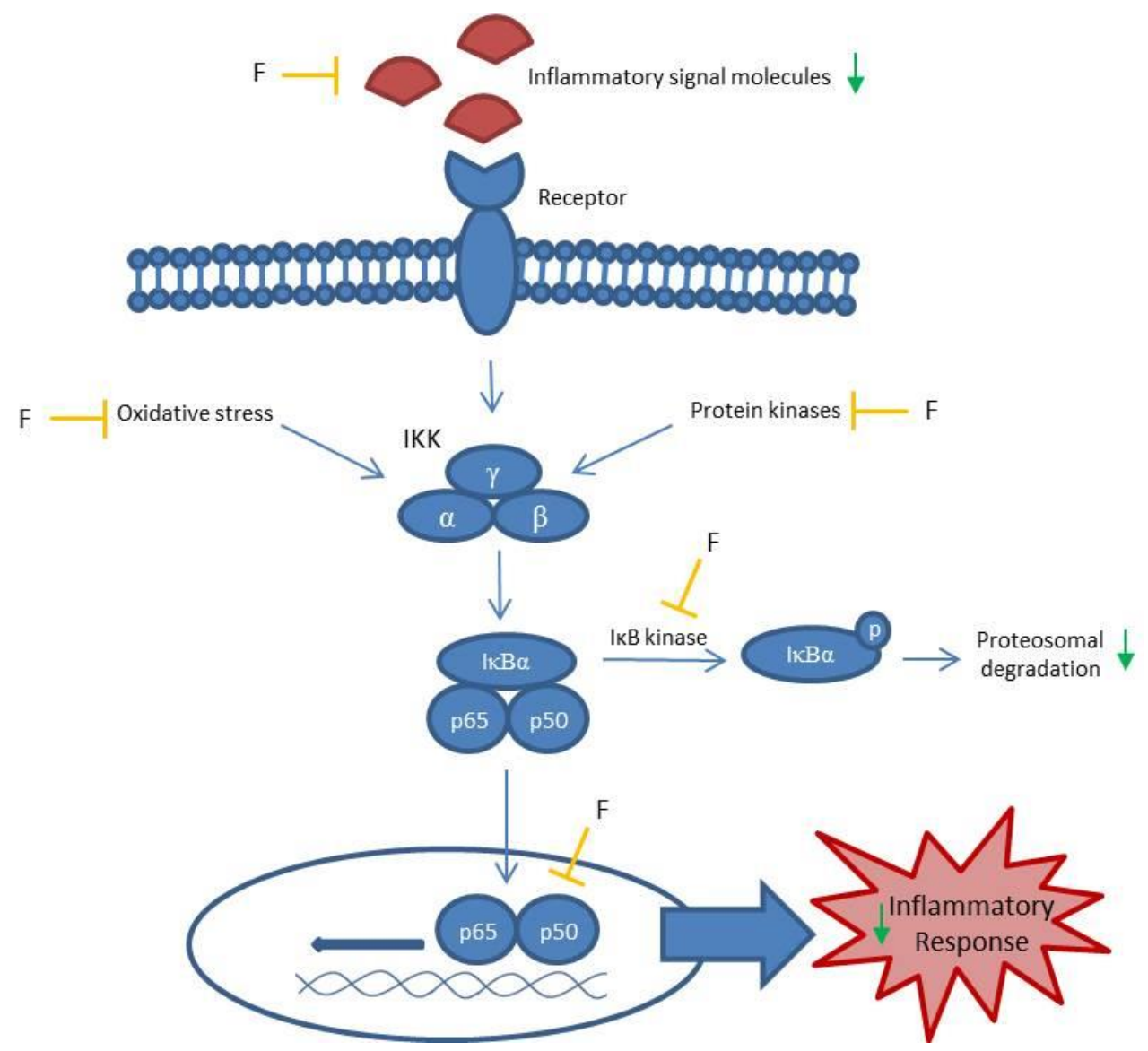

Figure 6. Effects of flavonoids on the NFкB-pathway. Inhibitory effects ( $-\mid$ ) of flavonoids (F) on different processes in the canonical NF- $\mathrm{B}$ pathway, leading to a decreased inflammatory response. Abbreviations: $\mathrm{IKK}=\mathrm{I} \kappa \mathrm{B}$ kinase, $\mathrm{I} \kappa \mathrm{B} \alpha=\mathrm{NF}-\kappa \mathrm{B}$ inhibitor $-\alpha, \mathrm{p} 65=\mathrm{NF}-\kappa \mathrm{B}$ complex subunit p65, p50 = NF- $\kappa \mathrm{B}$ complex subunit p50.

Besides inhibitory effects on the NF- $\kappa$ B pathway, flavonoids also inhibit the activity of regulatory enzymes involved in the induction of the inflammatory response, such as protein tyrosine kinases, $\mathrm{PKC}$, phosphodiesterase, phospholipase $\mathrm{A}_{2}$, lipoxygenases and cyclooxygenase (COX) $[63,64]$. These enzymes are responsible for the activation 
of specialized cells involved in inflammation, e.g. by prostanoid biosynthesis via arachidonic acid metabolism [64]. Also, the production of various cytokines is inhibited by flavonoids, possibly involving inhibition of phosphodiesterase $[64,68]$.

Furthermore, several flavonoids inhibit inducible nitric oxide synthase (iNOS) expression and the production of nitric oxide (NO) [63, 68]. NO serves as an inflammatory mediator and also leads to the formation of the highly damaging peroxynitrite in conditions of oxidative stress [44]. In addition to the inhibition of NO production, flavonoids can scavenge NO [69, 70] and peroxynitrite [47]. Inhibition of iNOS and COX-2 expression is also found to be related to inhibition of NF- $\mathrm{kB}$ and activation of PPAR $\gamma$ [68].

Recently, it has been reported that flavonoids are able to prevent deterioration of the anti-inflammatory effect of the glucocorticoid cortisol in the presence of oxidative stress [71]. Oxidative stress extinguishes the anti-inflammatory effect of cortisol, leading to cortisol resistance. Flavonoids reduce intracellular oxidative stress as well as the development of cortisol resistance. This further deciphers the enigmatic mechanism of flavonoids by which these bioactives exert their biological effect, and moreover shows that their anti-inflammatory and antioxidant action are intertwined.

\section{Flavonoids in the treatment of NAFLD}

Several flavonoids have been studied for the treatment of NAFLD. One of the most investigated flavonoids is silybin, one of the flavonoids in the flavonoid mixture silymarin. Green tea flavonoids and soy isoflavones are also extensively investigated. Together with quercetin and rutin, these are the most studied flavonoids for the treatment of NAFLD (figure 7). These groups of flavonoids and their effects on NAFLD are reviewed separately. 
<smiles>COc1cc(C2Oc3cc([C@@H]4Oc5cc(O)cc(O)c5C(=O)[C@H]4O)ccc3O[C@@H]2CO)ccc1O</smiles>

Silybin A<smiles>O=c1c(-c2ccc(O)cc2)coc2cc(O)cc(O)c12</smiles>

Genistein

Daidzein<smiles>O=C(O[C@H]1Cc2c(O)cc(O)cc2O[C@@H]1c1cc(O)c(O)c(O)c1)c1cc(O)c(O)c(O)c1</smiles><smiles>O=c1c(O)c(-c2ccc(O)c(O)c2)oc2cc(O)cc(O)c12</smiles>

Quercetin<smiles>O=c1c(-c2ccc(O)cc2)coc2cc(O)ccc12</smiles><smiles>C=C(O)C(=O)O</smiles><smiles>[R11][R17][R17]</smiles>

Figure 7. Most studied flavonoids in the treatment of NAFLD. Molecular structures of the most investigated flavonoids in the treatment of NAFLD: Silybin (mixture of two diateromers, of which one is pictured), genistein, daidzein, epigallocatechin-3-gallate (ECGC), quercetin and rutin.

\section{Animal models}

The majority of in vivo studies investigating the use of flavonoids in NAFLD are animal studies. Because NAFLD is a multifactorial disease and the patient population with NAFLD is very heterogenic, it is difficult to imitate all the facets of the disease in one animal model. Furthermore, NAFLD is seen as the hepatic manifestation of the metabolic syndrome: patients do not only show liver abnormalities, but also have obesity, dyslipidaemia and insulin resistance. Although many models may succeed to mirror the liver pathology correctly, this does not always reflect the right metabolic context [72].

Already many reviews have been devoted to animal models of NAFLD [73-75]. Therefore, this section will only evaluate the animal models used in the studies investigating the use of flavonoids in NAFLD. Most studies are mice or rat studies. Also one study investigated the development of NAFLD in gerbils. The animals used in the 
different studies had various genetic backgrounds and some transgenic animal models were used. Furthermore, different diets were used to induce NAFLD, such as high fat diets, high fructose diets and methionine- and choline deficient diets.

To validate the different models used, it has to be examined which model pictures the disease process most completely. Therefore, the models were compared regarding the development of liver damage (steatosis, inflammation and fibrosis), the presence of the most important pathogenic pathways (metabolic abnormalities, oxidative stress and inflammation) and the presence of other signs of the metabolic syndrome (dyslipidaemia, obesity and insulin resistance).

Firstly, the different dietary models are compared. The methionine and choline deficient diet (MCD diet) causes lipid deposition in the liver by interfering with $\beta$ oxidation and VLDL secretion. The diet lacks methionine and choline, which are essential for hepatic $\beta$-oxidation and the production of VLDL. In addition to liver steatosis and inflammation, oxidative stress and changes in the liver cytokines and adipocytokines are found [73]. The advantage of this model is that liver steatosis and inflammation are found within ten days of the diet. Fibrosis is found after 8-10 weeks [73]. The MCD-model causes the most inflammation, oxidative stress and liver damage compared to other dietary models. However, the extent of damage is dependent on species, gender and strain of the animals used [76]. C57Bl/6 mice are found to develop the most inflammation and necrosis, best approximating the histological features of NASH. Male gender and the strain Wistar rats are associated with the highest degree of steatosis [76]. The disadvantage of the MCD-model is that it lacks the metabolic context of human NAFLD/NASH. Animals on the MCD-diet are found to lose weight, to have no insulin resistance and unchanged or increased serum adiponectin levels [76]. In some studies this is resolved by using the MCD diet in genetically obese animals, such as ob/ob mice.

Various high fat diets have been used in animal models of NAFLD. Sprague-Dawley rats on a high fat diet are found to develop steatosis, inflammation and oxidative damage in the liver. Also insulin resistance is found after 3 weeks of a high fat diet [73]. However, development of steatohepatitis is dependent on the rodent species and strain used. For example, while Sprague-Dawley rats do develop steatohepatitis, Wistar rats were found to be less susceptible to develop steatohepatitis on a high fat diet [77]. C57Bl/6 mice are found to develop steatosis after 10 weeks of high fat diet. Also insulin resistance, increased plasma cholesterol levels and obesity are found. However, slight inflammatory changes were only found after 35 weeks of high fat diet 
[78]. The development of steatohepatitis in animals on a high fat diet is not only dependent on the rodent species and strain, but also on the fat content in the diet, the composition of dietary fat and the duration of the treatment [73]. Although the use of a high fat diet seems to reproduce the metabolic context of human NASH better, liver damage is less severe than with use of the MCD-diet. Among the various high fat diet models, the pathological changes in the intragastric-overfeeding model are found to resemble human NASH best [78].

Rats and mice fed a fructose rich diet have been found to be good models for the metabolic syndrome [73]. Liver damage is also found in these models. Wistar rats on a high fructose diet (70\%) develop liver steatosis and inflammation [79]. However, the distribution pattern of steatosis in the liver is different from that in human NAFLD. While in human NAFLD steatosis is mostly present in zone 3, the steatosis in Wistar rats on a high fructose diet is predominant in zone 1 [73]. Interestingly, inflammation does follow the same pattern as in human NASH: predominantly lobular and not periportal [79]. Like with the use of a high fat diet, the extent of liver damage developed on a high fructose diet is also dependent on the type and strain of animals used and the fructose content in the diet.

Transgenic animal models in studies investigating flavonoids in NAFLD include $\mathrm{db} / \mathrm{db}$ mice, ob/ob mice, nSREBP-1c transgenic mice, obese Zucker fa/fa rats and obese diabetic Otsuka Long-Evans Tokushima Fatty (OLETF) rats.

In ob/ob mice, a spontaneous mutation in the leptin gene causes leptin deficiency, leading to hyperphagic, inactive, extremely obese and diabetic mice that develop liver steatosis spontaneously $[73,74]$. However, ob/ob mice do not spontaneously develop steatohepatitis. Therefore, a second 'hit' is needed; such as a MCD diet or high fat diet $[73,74]$.

$\mathrm{Db} / \mathrm{db}$ mice carry a spontaneous mutation in the leptin-receptor gene. These mice have normal or increased levels of leptin, but are resistant to its effects, which leads to obesity and insulin resistance $[73,74]$. Db/db mice also develop steatosis, but require a second 'hit' for progression to steatohepatitis, such as the MCD diet or high fat diet $[73,74]$. The advantage of both the ob/ob and $\mathrm{db} / \mathrm{db}$ mice models is that they develop NAFLD in conditions resembling the metabolic syndrome. A disadvantage is that they need a second hit for progression to steatohepatitis. Db/db mice fed a MCD diet, were found to have higher serum ALT levels and more severe hepatic inflammation and fibrosis than ob/ob mice fed the MCD diet [73]. 
Obese Zucker fa/fa rats also have a mutation in the leptin receptor gene leading to leptin resistance. Until four weeks of age, these rats only display increased apatite [75]. At four to five weeks of age, the fat mass and the serum level of free fatty acids increase and triglycerides accumulate in various organs, including the liver [75]. Hyperinsulinemia also develops, eventually leading to diabetes. Development of steatohepatitis in Zucker fa/fa rats is described after feeding a high fat high cholesterol diet [80].

In SREBP-1c transgenic mice, SREBP-1c is overexpressed, causing congenital lipodystrophy and severe insulin resistance. At the age of 1 week, liver steatosis is found, which progresses to steatohepatitis within 20 weeks of age without the requirement of a second hit [73]. A disadvantage of this model is that in contrast with human NAFLD/NASH, visceral fat is decreased in this animal model.

The last genetic model used to investigate flavonoids in the treatment of NAFLD is the OLETF rat. This is an established model of the metabolic syndrome, characterized by insulin resistance, abdominal obesity, hypertension and dyslipidaemia [81]. Due to a gentic deletion of the cholecystokinin 1 receptor, these rats lack the feeling of satiety. From 8 weeks of age, the OLETF rats develop obesity and hyperinsulinemia. Also liver steatosis develops spontaneously in these rats at 18 weeks of age [81]. However, after 42 weeks of age steatosis declines and inflammation and fibrosis does not develop spontaneously [81].

From all the studies evaluated, the models using rats/gerbils on a high fat diet [82-84] (table 1), [85-87] (table 3), [88, 89] (table 4), [90] (table 5) or on a high fat diet combined with high fructose or carbohydrates [91, 92] (table 4 and 5), seem to approximate the human conditions of NAFLD best. Also studies using genetic models of the metabolic syndrome combined with a high fat diet or MCD diet are useful [65, 93, 94] (table 1). Studies using the MCD diet only, do not correctly mirror the circumstances of the metabolic syndrome. Studies using mice on a high fat diet, Wistar rats on only a high fat diet, or genetic models of the metabolic syndrome without a special diet or second 'hit', do not seem to provoke sufficient liver damage compared to the human situation and can only be used to evaluate the development of steatosis.

\section{Silymarin and silybin}

Silymarin is a flavonoid mix that originates from milk thistle extract. It was already used by doctors and herbalists to treat diverse liver and gallbladder disorders in 
ancient history [95]. Nowadays, $65 \%$ of the patients with liver diseases take herbal preparations, which are mainly derived from milk thistle [96]. Silymarin contains at least eight different compounds: silybin (A and B), isosilybin ( $A$ and B), silichristin, silidianin, dehydrosilybin, taxifolin and others. A small fraction of silymarin consists of polymerized polyphenolics that have not been identified yet [97]. About $50-70 \%$ of the silymarin extract consists of the flavonolignan silybin (figure 7), also known as silibinin, which is extensively studied and is regarded as the most active component of silymarin [98].

Silymarin flavanolignans have a limited bioavailability that was reported to be $0.45 \%$ in human volunteers [99]. Similarly, the bioavailability of silybin in rats was calculated to be $0.95 \%$ [100]. Extensive phase II metabolism, low permeability across intestinal epithelial cells, low solubility in water and rapid excretion in bile and urine are the major causes of this limited bioavailability [101]. To improve bioavailibility, derivatives of silybin have been synthesized, such as silybin-phosphatidylcholine [98, 101]. The silymarin that is not absorbed in the gastrointestinal tract will be subjected to metabolism by bacteria in the colon. In the biological effect of polyphenolic compounds, the effect of colonic metabolites plays a key role [102]. However, the contribution of the colonic metabolites to the health effect of silymarin has not been examined.

In human studies daily doses up to $800 \mathrm{mg}$ silymarin appeared to be safe. Only few and minor side effects of silymarin, primarily on the gastrointestinal tract, have been reported $[103,104]$.

The results of ten identified in vivo studies investigating the use of silymarin/silybin in NAFLD models are summarized in table 1 (supplementary data) [65, 82-84, 94, 105-109]. The molecular pathways implicated in the therapeutic effect of silymarin in NAFLD, are elaborated in detail in table 1, clustered in metabolic abnormalities, oxidative stress and inflammation. An improvement of lipid metabolism is demonstrated by a decrease in serum and hepatic lipid values in numerous studies. This is caused by a stimulation of free fatty acid oxidation and positive effects on coordinating factors of lipid metabolism, such as adiponectin and PPAR $\alpha$. Also insulin resistance is reduced in most studies. Antioxidant effects of silymarin represented by a decrease in lipid peroxidation and other oxidative stress markers might be caused by stimulation of endogenous antioxidants such as GSH and SOD. The antiinflammatory effects of silymarin in NAFLD models include a decrease in the proinflammatory cytokine TNF- $\alpha$ and inhibition of NF- $\kappa$ B. Together, the metabolic, 
antioxidant and anti-inflammatory effects of silymarin lead to a marked improvement of steatosis and liver inflammation in in vivo models of NAFLD (table 1).

In a well-designed, randomized controlled trial (RCT), 138 patients with histologically proven NAFLD were treated with a silybin-phosphatidylcholine complex or placebo for 12 months (table 2, supplementary data) [96]. Treatment showed positive effects on serum ALT, AST and $\gamma$ GT levels, suggesting an improvement of hepatic damage. In addition, positive effects on insulin resistance and body mass index were found. Patients of the treatment group that agreed to liver biopsy showed significant improvements in steatosis, lobular inflammation, ballooning and fibrosis, while no improvements were seen in the biopsies of patients in the placebo group [96].

Taken together, the data indicate that silymarin and silybin show inhibitory effects on NAFLD progression. The influence of silymarin/silybin on NAFLD seems to be in line with the multifactorial mode of action of flavonoids; not one single mechanism of action, but multiple mechanisms that reinforce each other, emerge from the studies. The exact value of silymarin in the treatment of NAFLD still has to be established, but results so far are relatively consistent and encouraging.

\section{Soy isoflavones}

Unlike most flavonoids, isoflavones are not commonly found in a Western diet [110]. In fact, soybeans and products derived from soybeans are the only relevant sources of isoflavones [110]. Isoflavone intake in Western countries does not exceed $1 \mathrm{mg}$ per day, whereas consumption of isoflavones in Japan and China can be as high as 40 $\mathrm{mg} /$ day [111, 112]. The isoflavones in soy are genistein (figure 7), daidzein (fig. 7) and glycitein. Generally, the content of genistein in soy is larger than the content of daidzein and glycitein [113].

Five to seven hours after intake, genistein and daidzein plasma concentrations reach their maximum [114]. Plasma half-lives of genistein and daidzein aglycones are found to be 7 and 9 hours respectively [113]. Due to extensive first-pass metabolism, isoflavone bioavailability is low [113]. Plasma genistein concentrations around $40 \mathrm{nM}$ are measured in people consuming a Western diet, while concentrations of approximately $4 \mu \mathrm{M}$ are measured in people consuming a traditional Japanese soybean rich diet [58]. 
Isoflavones are generally regarded as safe [115]. Most clinical trials do not report any adverse effects. Side effects that have been reported include abdominal bloating, constipation and hot flushes [115]. Isoflavones have estrogen-like activity in women with low endogenous estrogen levels [116]. Due to the estrogen-like activity of isoflavones, concerns were raised that isoflavones could stimulate breast cancer development in postmenopausal women. This is an issue of major concern; however, the limited studies in humans that addressed this subject did not corroborate this serious side effect [116].

Thirteen in vivo studies investigating the use of soy isoflavones in animal models of NAFLD were found [85-87, 117-126]. The various effects of soy isoflavones on metabolic abnormalities, oxidative stress and inflammation are shown in detail in table 3 (supplementary data). Improvement of lipid metabolism, evidenced by a decrease in hepatic and serum lipid values, has been attributed to stimulation of free fatty acid oxidation, inhibition of lipogenesis and interaction of soy isoflavones with coordinating factors of lipid metabolism, such as adiponectin, leptin, PPAR $\alpha$, PPAR $\gamma$ and others (table 3). Protection against oxidative stress is demonstrated by a decrease in lipid peroxidation and protein carbonyl levels. The mechanism proposed for this protection is stimulation of endogenous antioxidant levels. Inflammation is mitigated by inhibition of NF- $\mathrm{KB}$ activation and reduced production of pro-inflammatory cytokines (table 3). All these subtle effects combined can lead to a decrease in steatosis. The few studies that have investigated histological signs of inflammation corroborate the anti-inflammatory effect of isoflavones.

Since isoflavones inhibit many of the pathogenic mechanisms, they are promising compounds for the treatment of NAFLD. Although several animal studies have found positive effects of the use of soy isoflavones in the treatment of NAFLD, no clinical trials have been conducted on the use of soy isoflavones in NAFLD patients.

\section{Green tea flavonoids}

Green tea, similar to oolong tea and black tea, is derived from the plant Camellia Sinensis [127]. The main flavonoids in green tea are the catechins $(30-42 \%$ of solid weight). They comprise epicatechin (EC), epicatechin gallate (ECG), epigallocatechin (EGC) and epigallocatechin-gallate (EGCG) [127, 128]. The highest percentage of catechins in green tea consists of EGCG (50-75\%) (figure 7). Other flavonoids that can be found in small amounts in green tea are quercetin and myricitin [127]. 
One to three hours after ingestion of green tea, maximal catechin plasma concentrations (0.1-4.4 $\mu \mathrm{M})$ are reached [127]. Plasma half-lives of green tea catechins range from 1.5 to 5.7 hours [127]. Similar values were obtained in studies investigating pure EGCG [129]. Because of the rapid elimination of catechins from the body, intake of tea catechins might be beneficial only when they are consumed several times a day [127].

In some studies, concerns were raised regarding the possible hepatotoxicity of green tea extract (GTE) [130]. Nevertheless, a review of several clinical trials showed that the use of GTE/EGCG up to doses of $800 \mathrm{mg} / \mathrm{kg} /$ day is safe and well tolerated in humans [130]. The only side effects that were reported were mild headache and fatigue. In a recent clinical trial, daily consumption of $714 \mathrm{mg}$ GTE/day for 3 weeks did not lead to liver toxicity in healthy males [131].

Ten studies investigating the use of GTE or EGCG in animal models of NAFLD were found [88, 89, 132-139]. The effects of GTE/EGCG on metabolic abnormalities, oxidative stress and inflammation are presented in detail in table 4 (supplementary data). Improvement of lipid metabolism, represented by a decrease in serum and hepatic lipid levels, might be caused by interaction of green tea catechins with coordinating factors of lipid metabolism or a decrease in lipogenesis (table 4). Stimulation of antioxidants and inhibition of ROS production by green tea catechins leads to an attenuation of oxidative stress, demonstrated by a decrease in lipid peroxidation (table 4). Also inflammation is reduced by green tea catechins by inhibition of NF- $\mathrm{KB}$ activity and a decrease in pro-inflammatory cytokines (table 4). In the majority of studies these effects led to a decrease in steatosis. Only one study did not find a reduction of steatosis [136]. Histological inflammation was only investigated in two studies [89, 135]. Both studies found a diminution of inflammation.

Unfortunately, no studies investigating GTE/EGCG in NAFLD patients were found. In addition, most of the investigated animal studies focused on steatosis only. Although in general promising effects of GTE/EGCG on steatosis are reported, the effects of GTE/EGCG on inflammation in NAFLD models are not conclusive. Clinical trials are lacking to substantiate the therapeutic potential of green tea catechins in NAFLD. Furthermore, the rapid elimination from the body seriously questions the prospect of the use of green tea in NAFLD treatment. 


\section{Quercetin}

Quercetin (figure 7) is a flavonol that is one of the most abundant flavonoids in the human diet. It is found in various fruits and vegetables, such as onions, apples and tomatoes. The average intake of quercetin is estimated to be $5-40 \mathrm{mg} /$ day [140]. In the diet quercetin is often bound by sugars (quercetin glycosides).

Quercetin has a relatively high bioavailability compared to other flavonoids [141]. The bioavailability depends on the type of quercetin glycoside. It has been reported that glucosides of quercetin (quercetin bound by glucose) are absorbed in the gastrointestinal tract better than the aglycon [142, 143]. Fifty-two percent of quercetin glucosides from onions are absorbed in the gastro-intestinal tract, versus $24 \%$ of the quercetin aglycone [142]. Quercetin and its metabolites have long plasma half-lives, i.e. 11-28h [129]. Therefore, plasma concentrations can increase significantly upon frequent intake. Quercetin is normally found in the human plasma in low nano molar concentrations, but upon supplementation, this can increase to high nano molar or low micro molar concentrations $[144,145]$.

No adverse effects of quercetin were reported in studies investigating oral intake of quercetin or quercetin glucosides in doses of $3-1000 \mathrm{mg} /$ day for periods up to 12 weeks [146]. Also for intravenous doses of $\pm 10.8 \mathrm{mg} / \mathrm{kg}$ body weight no adverse effects were reported. For higher intravenous doses up to $51.3 \mathrm{mg} / \mathrm{kg}$ body weight, pronounced pain at the injection site, dyspnoea, emesis and transient nephrotoxicity were reported [146]. Clinical symptoms lasted for a period of time after each injection [146].

Although in vitro toxicity studies have reported mutagenic effects of quercetin and two in vivo animal studies reported quercetin carcinogenicity, many other animal studies fail to show increased tumor incidence related to quercetin administration [146]. Quercetin toxicity is assumed to be related to the formation of the quercetinquinone, which is produced when quercetin is oxidized by radicals $[67,147]$. The quercetin-quinone binds to thiols and causes toxic effects like increased membrane permeability and altered function of enzymes with a critical sulfhydryl -group [67, 147].

Six animal studies investigating the use of quercetin in animal models of NAFLD were identified [90, 92, 148-151]. Effects of quercetin on lipid metabolism, oxidative stress and inflammation are summarized in table 5 (supplementary data). Quercetin 
improves lipid metabolism by affecting coordinating factors of lipid metabolism, such as PPAR $\alpha$, PPAR $\gamma$ and adiponectin. Inhibition of lipogenesis and stimulation of fatty acid oxidation by quercetin also contribute to the improvement of lipid metabolism (table 5). Reduction of oxidative stress, demonstrated by a decrease in lipid peroxidation, is caused by a potent direct antioxidant effect, induction of endogenous antioxidant defences and inhibition of iNOS expression in the liver (table 5). In only two studies inflammatory markers, such as TNF- $\alpha$ and Il-6, were investigated and found to be decreased by quercetin (table 5). In all studies the quercetin administration led to a decrease in steatosis. In general, histological signs of liver inflammation were also reduced. Two studies that investigated fibrosis, found a reduction in fibrosis as well.

In the in vivo studies mainly positive effects of quercetin on NAFLD were demonstrated. A randomized clinical trial on the use of quercetin in NAFLD patients is lacking, whereas various clinical trials have investigated the use of quercetin in other diseases [152-156]. To substantiate the therapeutic effect of quercetin in NAFLD, clinical trials are mandatory. A concern for approval of such studies will be the potential carcinogenic effect of quercetin, though the relatively high intake of quercetin in the normal diet as well as the widely applied supplementation of quercetin have not pinpointed this as a problem. In fact, epidemiological studies reveal the opposite: flavonoid intake, which for the substantial part is quercetin, has an inverse relationship with the incidence of cancer.

\section{Rutin}

Rutin is quercetin with the disaccharide rutinose covalently bound to the 3-OH group (quercetin-3-0- $\beta$-rutinoside) (figure 7). It is abundantly found in plants such as buckwheat (Fagopyrum Esculentum) and citrus fruits such as oranges (Citrus Sinensis) and grapefruits (Citrus Paradisi) [32]. The uptake of rutin in the gastrointestinal tract is less than that of quercetin and quercetin mono-glucosides and lower peak plasma concentrations of rutin are reached after intake of an equivalent dose compared to that of quercetin [142, 143]. The main reason for rutins poor bioavailability is its poor solubility in aqueous media [32] and the resulting low bioaccessibility. However, rutin also has advantages over other flavonoids. It is relatively stable and does not display prominent pro-oxidant activity. While some flavonoids are labeled as mutagenic and relatively cytotoxic, rutin is neither [32]. 
Four studies examining the use of rutin in animal models of NAFLD were found [91, 157-159]. The effects of rutin on metabolism, oxidative stress and inflammation are presented in table 6 (supplementary data). Hepatic and/or serum lipid values were decreased in all studies, demonstrating an improvement in lipid metabolism. The mechanism of action was not investigated extensively in the studies. Inhibition of leptin and SREBP-1c was suggested to play a role (table 6). Only two studies examined the effects of rutin on oxidative stress. Both studies concluded that rutin administration reduced oxidative stress and implied as mechanism stimulation of endogenous antioxidant levels and inhibition of the production of ROS (table 6). One study reported that rutin administration reduced inflammatory markers, such as TNF$\alpha$ (table 6) [157]. The combined effects of rutin on metabolism, oxidative stress and inflammation, can explain the decrease in steatosis found in all studies. Liver inflammation was investigated in two studies and found to be reduced in both studies $[91,159]$. The only study that examined the effect of rutin on liver fibrosis, did find a reduction in fibrosis [91].

Rutin is not extensively investigated in animal models of NAFLD. Also no clinical trials have been performed. The promising results for the use of rutin against NAFLD summarized in table 6 show the potential of rutin and indicate that further studies are warranted. A disadvantage of rutin is its poor bioavailability. Semisynthetic analogues of rutin with increased bioavailability might be more promising. In monoHER, a hydroxyethyl group is attached to the oxygen to the 7-OH group, which increases the water solubility. MonoHER is proven to be a very potent antioxidant $[51,69,160,161]$ with anti-inflammatory characteristics [162].

\section{Other flavonoids}

A wide spectrum of flavonoids has been investigated in animal models of NAFLD. However, the number of studies on many flavonoids is limited to one or two studies. These flavonoids are presented in table 7 (supplementary data).

Naringenin, a flavanone found in grapefruit, completely reverses steatosis in LDLr $/$ mice fed a high fat diet. It also reduces dyslipidaemia, hyperglycaemia, hyperinsulinemia and body weight $[163,164]$.

Cyanidin 3-0- $\beta$-D-glucoside, an anthocyanin found in various plants and fruits that gives them a purple color, reduces hepatic steatosis, oxidative stress and inflammation in diabetic $\mathrm{db} / \mathrm{db}$ mice $[165,166]$. Furthermore, it reduces hyperglycemia and insulin 
resistance in $\mathrm{db} / \mathrm{db}$ mice as well as $\mathrm{C} 57 \mathrm{Bl} / 6 \mathrm{~J}$ mice fed a high fat diet [166]. Cyanidin 3O- $\beta$-D-glucoside also decreases body weight and hepatic lipid content in C57Bl/6J mice [167].

Xanthohumol, a chalcone from the hop plant (Humulus Lupulus), decreases steatosis, inflammation and fibrosis in murine models of NAFLD [168, 169]. Also the other flavonoids noted in table 7 were found to have positive effects on NAFLD and other factors contributing to NAFLD, such as dyslipidaemia, body weight and insulin resistance $[25,170-173]$.

Since the number of different flavonoids known is huge, up to more than 5,000 different chemical entities, it is mandatory to make a selection in order to keep clinical research feasible form a practical point of view. Although the miscellaneous flavonoids mentioned in this paragraph might have merit in the treatment of NAFLD, their biochemical profile is not that different form the more extensively investigated flavonoids. Therefore, we focus on the latter group of more extensively studied flavonoids in this review.

\section{Summary and perspective}

The contemporary pathophysiological model of NAFLD consists of multiple parallel pathways with a dynamic cross talk that cumulate in steatosis and inflammation and ultimately fibrosis, cirrhosis, liver failure and hepatocellular carcinoma. The multitude of pathways involved in the pathogenesis underpins the need for treatments that address these various pathways.

Flavonoids are compounds derived from plants with subtle effects on multiple targets that finally accumulate in a substantial health benefit. Interestingly, flavonoids have been found to have positive effects on lipid metabolism, insulin resistance, inflammation and oxidative stress, the most important pathological processes in the etiology of NAFLD. This puts flavonoids in the spotlight for the treatment of NAFLD. In this review the existing evidence for the use of flavonoids in the treatment of NAFLD is evaluated.

Flavonoids and flavonoid mixtures that have been widely investigated in animal models of NAFLD include silymarin, silybin, soy isoflavones, green tea flavonoids, quercetin and rutin. The protective biochemical profile of these flavonoids on lipid metabolism, insulin resistance, oxidative stress and inflammation as well as their 
beneficial therapeutic effect on steatosis and liver inflammation in most of the studies form the scientific fundament for the use of flavonoids in the treatment of NAFLD. However, further clinical studies are needed to examine the exact value of flavonoids in the treatment of NAFLD patients.

Further studies examining the use of flavonoids in NAFLD should include doubleblinded randomized clinical trials. In designing and interpreting clinical studies, it is of importance to carefully consider the heterogeneity of the NAFLD patient group. A caveat is that the high heterogeneity will negatively affect the power of the study. Moreover, NAFLD patients with a very different biochemical profile might benefit form flavonoids that display a biochemical profile that fits these different profiles the best. This means that it is unlikely that a uniform treatment for all types of NAFLD will be found. Personalized treatment with close monitoring of the therapeutic effect seems warranted to come to optimal treatment. To reach this stage, the most promising flavonoids should be tested first. The spectrum of flavonoids includes over 5,000 different compounds with their own unique profile, illustrating that it is impossible to study all the flavonoids. Therefore, the compounds best suitable for further investigation have to be identified. In identifying the most promising compound in a large series, structure-activity relationships are mandatory, although these relationships should always be critically evaluated [174]. Criteria that can be used to evaluate the therapeutic potential and to form a rational basis for selection of flavonoids for further investigation are their molecular mechanism of action and clinical evidence, bioavailability and safety.

Regarding their molecular mechanism of action, none of the flavonoids seems to protrude from the animal studies. All flavonoids have been found to improve lipid metabolism, insulin resistance, oxidative stress and inflammatory markers. However, in structure activity relationship studies, quercetin, rutin and its derivates are found to belong to the most potent antioxidants [48]. Most clinical evidence is found for silymarin and silybin because these compounds are widely investigated in animal models of NAFLD and silybin was also examined in a randomized clinical trial.

Regarding bioavailability, quercetin has the best characteristics. Bioavailability of rutin is slightly lower, but can be improved by the use of semi-synthetic derivates, such as monoHER, which is more water-soluble. Bioavailability of silybin and silymarin is considerably lower and can be improved by conjugation to polar and hydrophilic moieties, e.g. as in silybin-phosphatidylcholine. Due to the rapid 
elimination from the body and consequent necessity of frequent administration, green tea flavonoids are not likely to get a place in NAFLD treatment.

Although quercetin appears to possess superior antioxidant potential and bioavailability, quercetins safety is still debated due to its potential carcinogenicity. Rutin, which seems to be devoid of carcinogenic properties but displays similar antioxidant potential and only slightly lower bioavailability as quercetin, seems to be a better option. Rutin derivates, developed to increase bioavailability, seem to be even more appealing compounds for further investigation. For example monoHER, rutin with a hydroxyethyl group attached to the oxygen on the 7-position, has shown to be a very potent antioxidant $[50,51]$.

In conclusion, with their multifaceted actions flavonoids seem to suit perfectly in the pathophysiological model of NAFLD. The heterogeneity of the disease should be carefully considered in the design of clinical studies investigating a treatment for NAFLD. Already, multiple in vivo studies show encouraging results for the use of flavonoids in the treatment of NAFLD, which calls for additional research. Silybin has the advantage that it is the most studied flavonoid. Nevertheless, rutin and its derivatives, such as monoHER, seem to be the most appealing flavonoids for further investigation due to their high antioxidant potential, bioavailability and safety. 


\section{References}

1. Bedogni, G., et al., Prevalence of and risk factors for nonalcoholic fatty liver disease: The Dionysos nutrition and liver study. Hepatology, 2005. 42(1): p. 44-52.

2. Neuschwander-Tetri, B.A. and S.H. Caldwell, Nonalcoholic steatohepatitis: Summary of an AASLD Single Topic Conference. Hepatology, 2003. 37(5): p. 1202-1219.

3. Tilg, H. and A.R. Moschen, Evolution of inflammation in nonalcoholic fatty liver disease: the multiple parallel hits hypothesis. Hepatology, 2010. 52(5): p. 1836-46.

4. Yilmaz, Y., Review article: is non-alcoholic fatty liver disease a spectrum, or are steatosis and nonalcoholic steatohepatitis distinct conditions? Alimentary pharmacology \& therapeutics, 2012. 36(9): p. 815-23.

5. Pais, R., et al., Progression from isolated steatosis to steatohepatitis and fibrosis in nonalcoholic fatty liver disease. Clinics and research in hepatology and gastroenterology, 2011. 35(1): p. 23-28.

6. Bast, A. and G.R. Haenen, Ten misconceptions about antioxidants. Trends in pharmacological sciences, 2013. 34(8): p. 430-6.

7. Polyzos, S.A., et al., Nonalcoholic fatty liver disease: multimodal treatment options for a pathogenetically multiple-hit disease. Journal of clinical gastroenterology, 2012. 46(4): p. 272-84.

8. Bieghs, V., et al., Trapping of oxidized LDL in lysosomes of Kupffer cells is a trigger for hepatic inflammation. Liver international : official journal of the International Association for the Study of the Liver, 2013. 33(7): p. 1056-61.

9. Koek, G.H., P.R. Liedorp, and A. Bast, The role of oxidative stress in non-alcoholic steatohepatitis. Clin Chim Acta, 2011. 412(15-16): p. 1297-1305.

10. Rolo, A.P., J.S. Teodoro, and C.M. Palmeira, Role of oxidative stress in the pathogenesis of nonalcoholic steatohepatitis. Free Radic Biol Med, 2012. 52: p. 59-69.

11. Ucar, F., et al., The relationship between oxidative stress and nonalcoholic fatty liver disease: Its effects on the development of nonalcoholic steatohepatitis. Redox report : communications in free radical research, 2013.18(4): p. 127-33.

12. Nelson, J.E., et al., Relationship between the pattern of hepatic iron deposition and histological severity in non alcoholic fatty liver disease. Hepatology, 2011. 53(2): p. 448-457.

13. Valenti, L., et al., HFE genotype, parenchymal iron accumulation, and liver fibrosis in patients with nonalcoholic fatty liver disease. Gastroenterology, 2010. 138(3): p. 905-912.

14. Fenton, H.J.H., Oxidation of tartaric acid in presence of iron. J Chem Soc Trans, 1894. 65: p. 899911.

15. van de Wier, B., et al., Elevated citrate levels in non-alcoholic fatty liver disease: the potential of citrate to promote radical production. FEBS letters, 2013. 587(15): p. 2461-6.

16. Ross, J.A. and C.M. Kasum, Dietary flavonoids: bioavailability, metabolic effects, and safety. Annual review of nutrition, 2002. 22: p. 19-34.

17. Tailleux, A., K. Wouters, and B. Staels, Roles of PPARs in NAFLD: potential therapeutic targets. Biochimica et biophysica acta, 2012. 1821(5): p. 809-18.

18. Kallwitz, E.R., A. McLachlan, and S.J. Cotler, Role of peroxisome proliferators-activated receptors in the pathogenesis and treatment of nonalcoholic fatty liver disease. World journal of gastroenterology : WJG, 2008.14(1): p. 22-8.

19. Macdonald, G.A. and J.B. Prins, Peroxisomal fatty acid metabolism, peroxisomal proliferatoractivated receptors and non-alcoholic fatty liver disease. Journal of gastroenterology and hepatology, 2004. 19(12): p. 1335-7.

20. Shiri-Sverdlov, R., et al., Early diet-induced non-alcoholic steatohepatitis in APOE2 knock-in mice and its prevention by fibrates. Journal of Hepatology, 2006. 44(4): p. 732-741.

21. Chang, C.J., et al., Kaempferol regulates the lipid-profile in high-fat diet-fed rats through an increase in hepatic PPARalpha levels. Planta medica, 2011. 77(17): p. 1876-82. 


\section{Chapter 3}

22. Cho, K.W., et al., Dietary naringenin increases hepatic peroxisome proliferators-activated receptor alpha protein expression and decreases plasma triglyceride and adiposity in rats. European journal of nutrition, 2011. 50(2): p. 81-8.

23. Goto, T., et al., Tiliroside, a glycosidic flavonoid, ameliorates obesity-induced metabolic disorders via activation of adiponectin signaling followed by enhancement of fatty acid oxidation in liver and skeletal muscle in obese-diabetic mice. The Journal of nutritional biochemistry, 2012. 23(7): $\mathrm{p}$. 768-776.

24. Jia, Y., et al., Cyanidin is an agonistic ligand for peroxisome proliferator-activated receptor-alpha reducing hepatic lipid. Biochimica et biophysica acta, 2013. 1831(4): p. 698-708.

25. Lee, J.W., et al., AMPK activation with glabridin ameliorates adiposity and lipid dysregulation in obesity. Journal of lipid research, 2012. 53(7): p. 1277-86.

26. Malek, M.A., et al., Ombuin-3-O-beta-D-glucopyranoside from Gynostemma pentaphyllum is a dual agonistic ligand of peroxisome proliferator-activated receptors alpha and delta/beta. Biochemical and biophysical research communications, 2013. 430(4): p. 1322-8.

27. Medjakovic, S., M. Mueller, and A. Jungbauer, Potential health-modulating effects of isoflavones and metabolites via activation of PPAR and AhR. Nutrients, 2010. 2(3): p. 241-79.

28. Savage, D.B., et al., Human metabolic syndrome resulting from dominant-negative mutations in the nuclear receptor peroxisome proliferator-activated receptor-gamma. Diabetes, 2003. 52(4): $\mathrm{p}$. 910-7.

29. Puhl, A.C., et al., Mode of peroxisome proliferator-activated receptor gamma activation by luteolin. Molecular pharmacology, 2012. 81(6): p. 788-99.

30. Chen, N., et al., Green tea, black tea, and epigallocatechin modify body composition, improve glucose tolerance, and differentially alter metabolic gene expression in rats fed a high-fat diet. Nutrition research, 2009. 29(11): p. 784-93.

31. Lee, Y.-S., et al., Nobiletin improves obesity and insulin resistance in high-fat diet-induced obese mice. The Journal of nutritional biochemistry, 2013. 24(1): p. 156-162.

32. Sharma, S., et al., Rutin : therapeutic potential and recent advances in drug delivery. Expert opinion on investigational drugs, 2013.

33. Xia, M., et al., Anthocyanins induce cholesterol efflux from mouse peritoneal macrophages: the role of the peroxisome proliferator-activated receptor $\{$ gamma\}-liver X receptor \{alpha\}-ABCA1 pathway. The Journal of biological chemistry, 2005. 280(44): p. 36792-801.

34. Sharma, A.K., et al., Up-regulation of PPARgamma, heat shock protein-27 and -72 by naringin attenuates insulin resistance, beta-cell dysfunction, hepatic steatosis and kidney damage in a rat model of type 2 diabetes. The British journal of nutrition, 2011. 106(11): p. 1713-23.

35. Ferre, P. and F. Foufelle, Hepatic steatosis: a role for de novo lipogenesis and the transcription factor SREBP-1c. Diabetes, obesity \& metabolism, 2010. 12 Suppl 2: p. 83-92.

36. Higuchi, N., et al., Liver X receptor in cooperation with SREBP-1c is a major lipid synthesis regulator in nonalcoholic fatty liver disease. Hepatology research : the official journal of the Japan Society of Hepatology, 2008. 38(11): p. 1122-9.

37. Ahn, T.G., et al., Molecular mechanisms underlying the anti-obesity potential of prunetin, an $O$ methylated isoflavone. Biochemical pharmacology, 2013. 85(10): p. 1525-33.

38. Hwang, Y.P., et al., Purple sweet potato anthocyanins attenuate hepatic lipid accumulation through activating adenosine monophosphate-activated protein kinase in human HepG2 cells and obese mice. Nutrition research, 2011. 31(12): p. 896-906.

39. Liu, J.F., et al., Reduction of lipid accumulation in HepG2 cells by luteolin is associated with activation of AMPK and mitigation of oxidative stress. Phytotherapy research : PTR, 2011. 25(4): p. 588-96.

40. Shin, E.S., et al., Genistein downregulates SREBP-1 regulated gene expression by inhibiting site-1 protease expression in HepG2 cells. The Journal of nutrition, 2007. 137(5): p. 1127-31.

41. $\quad \mathrm{Wu}, \mathrm{C} . \mathrm{H}$. , et al., Rutin inhibits oleic acid induced lipid accumulation via reducing lipogenesis and oxidative stress in hepatocarcinoma cells. Journal of Food Science, 2011. 76(2): p. T65-72. 
42. Yap, F., L. Craddock, and J. Yang, Mechanism of AMPK suppression of LXR-dependent Srebp-1c transcription. International journal of biological sciences, 2011. 7(5): p. 645-50.

43. Goldwasser, J., et al., Transcriptional regulation of human and rat hepatic lipid metabolism by the grapefruit flavonoid naringenin: role of PPARalpha, PPARgamma and LXRalpha. PloS one, 2010. 5(8): p. e12399.

44. Garcia-Lafuente, A., et al., Flavonoids as anti-inflammatory agents: implications in cancer and cardiovascular disease. Inflammation research : official journal of the European Histamine Research Society ... [et al.], 2009. 58(9): p. 537-52.

45. Duthie, G. and A. Crozier, Plant-derived phenolic antioxidants. Current opinion in lipidology, 2000. 11(1): p. 43-7.

46. Bors, W., C. Michel, and K. Stettmaier, Antioxidant effects of flavonoids. BioFactors, 1997. 6(4): p. 399-402.

47. Haenen, G.R.M.M., et al., Peroxynitrite Scavenging by Flavonoids. Biochemical and biophysical research communications, 1997. 236(3): p. 591-593.

48. Heijnen, C.G., et al., Protection of flavonoids against lipid peroxidation: the structure activity relationship revisited. Free radical research, 2002. 36(5): p. 575-81.

49. Pietta, P.G., Flavonoids as antioxidants. Journal of natural products, 2000. 63(7): p. 1035-42.

50. Lemmens, K.J.A., et al., The flavonoid 7-mono-O-( $\beta$-hydroxyethyl)-rutoside is able to protect endothelial cells by a direct antioxidant effect. Toxicology in vitro, in press.

51. Haenen, G.R.M.M., F.P. Jansen, and A. Bast, The antioxidant properties of five O-( $\beta$-hydroxyethyl)rutosides of the flavonoid mixture venoruton. Phlebology, 1993. 8(suppl 1): p. 10-17.

52. O'Brien, J. and L.P. Powell, Non-alcoholic fatty liver disease: Is iron relevant? Hepatol Int, 2011. 6(1): p. 332-341.

53. Stevenson, D.E. and R.D. Hurst, Polyphenolic phytochemicals--just antioxidants or much more? Cellular and molecular life sciences : CMLS, 2007. 64(22): p. 2900-16.

54. Sun, G.B., et al., Oxidative stress suppression by luteolin-induced heme oxygenase-1 expression. Toxicology and applied pharmacology, 2012. 265(2): p. 229-40.

55. Huang, C.S., et al., Protection by chrysin, apigenin, and luteolin against oxidative stress is mediated by the Nrf2-dependent up-regulation of heme oxygenase 1 and glutamate cysteine ligase in rat primary hepatocytes. Archives of toxicology, 2013. 87(1): p. 167-78.

56. Zhang, Z., et al., Baicalein protects against 6-OHDA-induced neurotoxicity through activation of Keap1/Nrf2/HO-1 and involving PKCalpha and PI3K/AKT signaling pathways. Journal of agricultural and food chemistry, 2012. 60(33): p. 8171-82.

57. Yang, Y.C., et al., Induction of glutathione synthesis and heme oxygenase 1 by the flavonoids butein and phloretin is mediated through the ERK/Nrf2 pathway and protects against oxidative stress. Free radical biology \& medicine, 2011. 51(11): p. 2073-81.

58. Mann, G.E., et al., Targeting the redox sensitive Nrf2-Keap1 defense pathway in cardiovascular disease: protection afforded by dietary isoflavones. Current opinion in pharmacology, 2009. 9(2): p. 139-45.

59. Gonzalez, R., et al., Effects of flavonoids and other polyphenols on inflammation. Critical reviews in food science and nutrition, 2011. 51(4): p. 331-62.

60. Shih, V.F., et al., A single NFkappaB system for both canonical and non-canonical signaling. Cell research, 2011. 21(1): p. 86-102.

61. van den Berg, R., et al., Transcription factor NF-kappaB as a potential biomarker for oxidative stress. The British journal of nutrition, 2001. 86 Suppl 1: p. S121-7.

62. Marra, F., Nuclear factor-kappaB inhibition and non-alcoholic steatohepatitis: inflammation as a target for therapy. Gut, 2008. 57(5): p. 570-2.

63. Kim, H., et al., Metabolic and pharmacological properties of rutin, a dietary quercetin glycoside, for treatment of inflammatory bowel disease. Pharmaceutical research, 2005. 22(9): p. 1499-509.

64. Manthey, J.A., Biological properties of flavonoids pertaining to inflammation. Microcirculation, 2000. 7(6 Pt 2): p. S29-34. 


\section{Chapter 3}

65. Salamone, F., et al., Silibinin modulates lipid homeostasis and inhibits nuclear factor kappa $B$ activation in experimental nonalcoholic steatohepatitis. Translational research : the journal of laboratory and clinical medicine, 2012. 159(6): p. 477-86.

66. Gloire, G. and J. Piette, Redox regulation of nuclear post-translational modifications during NFkappaB activation. Antioxidants \& redox signaling, 2009. 11(9): p. 2209-22.

67. Boots, A.W., G.R. Haenen, and A. Bast, Health effects of quercetin: from antioxidant to nutraceutical. European journal of pharmacology, 2008. 585(2-3): p. 325-37.

68. Gonzalez-Gallego, J., et al., Fruit polyphenols, immunity and inflammation. The British journal of nutrition, 2010. 104 Suppl 3: p. S15-27.

69. van Acker, S.A., et al., Flavonoids as scavengers of nitric oxide radical. Biochemical and biophysical research communications, 1995. 214(3): p. 755-9.

70. Haenen, G.R. and A. Bast, Nitric oxide radical scavenging of flavonoids. Methods in enzymology, 1999. 301: p. 490-503.

71. Ruijters, E.J.B., et al., The cocoa flavanol (-)-epicatechin protects the cortisol response. Pharmacological Research, 2014. 79(0): p. 28-33.

72. Larter, C.Z. and M.M. Yeh, Animal models of NASH: getting both pathology and metabolic context right. Journal of gastroenterology and hepatology, 2008. 23(11): p. 1635-48.

73. Takahashi, Y., Y. Soejima, and T. Fukusato, Animal models of nonalcoholic fatty liver disease/nonalcoholic steatohepatitis. World journal of gastroenterology : WJG, 2012. 18(19): p. 2300-8.

74. Nagarajan, P., et al., Genetically modified mouse models for the study of nonalcoholic fatty liver disease. World journal of gastroenterology : WJG, 2012. 18(11): p. 1141-53.

75. Nanji, A.A., Animal models of nonalcoholic fatty liver disease and steatohepatitis. Clinics in liver disease, 2004. 8(3): p. 559-74, ix.

76. Kirsch, R., et al., Rodent nutritional model of non-alcoholic steatohepatitis: species, strain and sex difference studies. Journal of gastroenterology and hepatology, 2003. 18(11): p. 1272-82.

77. Romestaing, C., et al., Long term highly saturated fat diet does not induce NASH in Wistar rats. Nutrition \& metabolism, 2007. 4: p. 4.

78. Ito, M., et al., Longitudinal analysis of murine steatohepatitis model induced by chronic exposure to high-fat diet. Hepatology research : the official journal of the Japan Society of Hepatology, 2007. 37(1): p. 50-7.

79. Kawasaki, T., et al., Rats fed fructose-enriched diets have characteristics of nonalcoholic hepatic steatosis. The Journal of nutrition, 2009. 139(11): p. 2067-71.

80. Matsunami, T., et al., Regulation of oxidative stress and inflammation by hepatic adiponectin receptor 2 in an animal model of nonalcoholic steatohepatitis. International journal of clinical and experimental pathology, 2010. 3(5): p. 472-81.

81. Song, Y.S., et al., Time course of the development of nonalcoholic Fatty liver disease in the Otsuka long-evans Tokushima Fatty rat. Gastroenterology research and practice, 2013. 2013: p. 342648.

82. Haddad, Y., et al., Antioxidant and hepatoprotective effects of silibinin in a rat model of nonalcoholic steatohepatitis. Evidence-based complementary and alternative medicine : eCAM, 2011. 2011: p. nep164.

83. Yao, J., et al., Effect and the probable mechanisms of silibinin in regulating insulin resistance in the liver of rats with non-alcoholic fatty liver. Brazilian journal of medical and biological research $=$ Revista brasileira de pesquisas medicas e biologicas / Sociedade Brasileira de Biofisica ... [et al.], 2013. 46(3): p. 270-7.

84. Yao, J., M. Zhi, and C. Minhu, Effect of silybin on high-fat-induced fatty liver in rats. Brazilian journal of medical and biological research = Revista brasileira de pesquisas medicas e biologicas /

Sociedade Brasileira de Biofisica ... [et al.], 2011. 44(7): p. 652-9.

85. Ji, G., et al., Anti-inflammatory effect of genistein on non-alcoholic steatohepatitis rats induced by high fat diet and its potential mechanisms. International immunopharmacology, 2011. 11(6): p. 762-8. 
86. Ronis, M.J., et al., Dietary soy protein isolate attenuates metabolic syndrome in rats via effects on PPAR, LXR, and SREBP signaling. The Journal of nutrition, 2009. 139(8): p. 1431-8.

87. Yalniz, M., et al., Preventive role of genistein in an experimental non-alcoholic steatohepatitis model. Journal of gastroenterology and hepatology, 2007. 22(11): p. 2009-14.

88. Kuzu, N., et al., Epigallocatechin gallate attenuates experimental non-alcoholic steatohepatitis induced by high fat diet. Journal of gastroenterology and hepatology, 2008. 23(8 Pt 2): p. e465-70.

89. Xiao, J., et al., Epigallocatechin gallate attenuates fibrosis, oxidative stress, and inflammation in nonalcoholic fatty liver disease rat model through TGF/SMAD, PI3 K/Akt/FoxO1, and NF-kappa B pathways. European journal of nutrition, 2013.

90. Ying, H.Z., et al., Dietary quercetin ameliorates nonalcoholic steatohepatitis induced by a high-fat diet in gerbils. Food and chemical toxicology : an international journal published for the British Industrial Biological Research Association, 2013. 52: p. 53-60.

91. Panchal, S.K., et al., Rutin attenuates metabolic changes, nonalcoholic steatohepatitis, and cardiovascular remodeling in high-carbohydrate, high-fat diet-fed rats. The Journal of nutrition, 2011. 141(6): p. 1062-9.

92. Panchal, S.K., H. Poudyal, and L. Brown, Quercetin ameliorates cardiovascular, hepatic, and metabolic changes in diet-induced metabolic syndrome in rats. The Journal of nutrition, 2012. 142(6): p. 1026-32.

93. Kim, K.D., et al., Silibinin regulates gene expression, production and secretion of mucin from cultured airway epithelial cells. Phytotherapy research : PTR, 2012. 26(9): p. 1301-7.

94. Salamone, F., et al., Silibinin improves hepatic and myocardial injury in mice with nonalcoholic steatohepatitis. Digestive and liver disease : official journal of the Italian Society of Gastroenterology and the Italian Association for the Study of the Liver, 2012. 44(4): p. 334-42.

95. Abenavoli, L., N. Milic, and F. Capasso, Anti-oxidant therapy in non-alcoholic fatty liver disease: the role of silymarin. Endocrine, 2012. 42(3): p. 754-5.

96. Loguercio, C., et al., Silybin combined with phosphatidylcholine and vitamin E in patients with nonalcoholic fatty liver disease: a randomized controlled trial. Free radical biology \& medicine, 2012. 52(9): p. 1658-65.

97. Skottova, N., et al., Effects of polyphenolic fraction of silymarin on lipoprotein profile in rats fed cholesterol-rich diets. Pharmacological research : the official journal of the Italian Pharmacological Society, 2003. 47(1): p. 17-26.

98. Loguercio, C. and D. Festi, Silybin and the liver: from basic research to clinical practice. World journal of gastroenterology : WJG, 2011. 17(18): p. 2288-301.

99. Calani, L., et al., Absorption and metabolism of milk thistle flavanolignans in humans. Phytomedicine : international journal of phytotherapy and phytopharmacology, 2012. 20(1): p. 40-6.

100. Wu, J.W., L.C. Lin, and T.H. Tsai, Drug-drug interactions of silymarin on the perspective of pharmacokinetics. Journal of ethnopharmacology, 2009. 121(2): p. 185-93.

101. Javed, S., K. Kohli, and M. Ali, Reassessing bioavailability of silymarin. Alternative medicine review : a journal of clinical therapeutic, 2011. 16(3): p. 239-49.

102. Mateo Anson, N., et al., Bioprocessing of wheat bran in whole wheat bread increases the bioavailability of phenolic acids in men and exerts antiinflammatory effects ex vivo. The Journal of nutrition, 2011. 141(1): p. 137-43.

103. Gazak, R., D. Walterova, and V. Kren, Silybin and silymarin--new and emerging applications in medicine. Current medicinal chemistry, 2007. 14(3): p. 315-38.

104. Jacobs, B.P., et al., Milk thistle for the treatment of liver disease: a systematic review and metaanalysis. The American journal of medicine, 2002.113(6): p. 506-15.

105. Qin, R., et al., Protective effects of gypenosides against fatty liver disease induced by high fat and cholesterol diet and alcohol in rats. Archives of pharmacal research, 2012. 35(7): p. 1241-50.

106. Shetty, S.N., et al., A study of standardized extracts of Picrorhiza kurroa Royle ex Benth in experimental nonalcoholic fatty liver disease. Journal of Ayurveda and integrative medicine, 2010. 1(3): p. 203-10. 


\section{Chapter 3}

107. Serviddio, G., et al., A silybin-phospholipid complex prevents mitochondrial dysfunction in a rodent model of nonalcoholic steatohepatitis. The Journal of pharmacology and experimental therapeutics, 2010. 332(3): p. 922-32.

108. Kim, M., et al., Silymarin suppresses hepatic stellate cell activation in a dietary rat model of nonalcoholic steatohepatitis: analysis of isolated hepatic stellate cells. International journal of molecular medicine, 2012. 30(3): p. 473-9.

109. Grattagliano, I., et al., A silybin-phospholipids complex counteracts rat fatty liver degeneration and mitochondrial oxidative changes. World journal of gastroenterology : WJG, 2013. 19(20): p. 3007 17.

110. Messina, M., A brief historical overview of the past two decades of soy and isoflavone research. The Journal of nutrition, 2010. 140(7): p. 1350S-4S.

111. Messina, M., C. Nagata, and A.H. Wu, Estimated Asian adult soy protein and isoflavone intakes. Nutrition and cancer, 2006. 55(1): p. 1-12.

112. van Erp-Baart, M.A., et al., Isoflavone intake in four different European countries: the VENUS approach. The British journal of nutrition, 2003. 89 Suppl 1: p. S25-30.

113. Larkin, T., W.E. Price, and L. Astheimer, The key importance of soy isoflavone bioavailability to understanding health benefits. Critical reviews in food science and nutrition, 2008. 48(6): p. 53852.

114. Vitale, D.C., et al., Isoflavones: estrogenic activity, biological effect and bioavailability. European journal of drug metabolism and pharmacokinetics, 2013. 38(1): p. 15-25.

115. Qin, Y., et al., Isoflavones for hypercholesterolaemia in adults. The Cochrane database of systematic reviews, 2013. 6: p. CD009518.

116. Andres, S., et al., Risks and benefits of dietary isoflavones for cancer. Critical reviews in toxicology, 2011. 41(6): p. 463-506.

117. Ae Park, S., et al., Genistein and daidzein modulate hepatic glucose and lipid regulating enzyme activities in C57BL/KsJ-db/db mice. Life sciences, 2006. 79(12): p. 1207-13.

118. Crespillo, A., et al., Reduction of body weight, liver steatosis and expression of stearoyl-CoA desaturase 1 by the isoflavone daidzein in diet-induced obesity. British journal of pharmacology, 2011. 164(7): p. 1899-915.

119. Davis, J., et al., Soy protein and isoflavones influence adiposity and development of metabolic syndrome in the obese male ZDF rat. Annals of nutrition \& metabolism, 2007. 51(1): p. 42-52.

120. Gudbrandsen, O.A., et al., Dietary soya protein concentrate enriched with isoflavones reduced fatty liver, increased hepatic fatty acid oxidation and decreased the hepatic mRNA level of VLDL receptor in obese Zucker rats. The British journal of nutrition, 2006. 96(2): p. 249-57.

121. Kim, M.H., K.S. Kang, and Y.S. Lee, The inhibitory effect of genistein on hepatic steatosis is linked to visceral adipocyte metabolism in mice with diet-induced non-alcoholic fatty liver disease. The British journal of nutrition, 2010. 104(9): p. 1333-42.

122. Kim, M.H., et al., Daidzein supplementation prevents non-alcoholic fatty liver disease through alternation of hepatic gene expression profiles and adipocyte metabolism. International journal of obesity, 2011. 35(8): p. 1019-30.

123. Lee, Y.M., et al., Effects of dietary genistein on hepatic lipid metabolism and mitochondrial function in mice fed high-fat diets. Nutrition, 2006. 22(9): p. 956-64.

124. Mohamed Salih, S., et al., Genistein improves liver function and attenuates non-alcoholic fatty liver disease in a rat model of insulin resistance. Journal of diabetes, 2009. 1(4): p. 278-87.

125. Ustundag, B., et al., Protective effect of soy isoflavones and activity levels of plasma paraoxonase and arylesterase in the experimental nonalcoholic steatohepatitis model. Digestive diseases and sciences, 2007. 52(8): p. 2006-14.

126. Gudbrandsen, O.A., H. Wergedahl, and R.K. Berge, A casein diet added isoflavone-enriched soy protein favorably affects biomarkers of steatohepatitis in obese Zucker rats. Nutrition, 2009. 25(5): p. 574-80.

127. Masterjohn, C. and R.S. Bruno, Therapeutic potential of green tea in nonalcoholic fatty liver disease. Nutrition reviews, 2012. 70(1): p. 41-56. 
128. Paquay, J.B., et al., Protection against nitric oxide toxicity by tea. Journal of agricultural and food chemistry, 2000. 48(11): p. 5768-72.

129. Manach, C., et al., Bioavailability and bioefficacy of polyphenols in humans. I. Review of 97 bioavailability studies. The American journal of clinical nutrition, 2005. 81(1 Suppl): p. 230S242S.

130. Sarma, D.N., et al., Safety of green tea extracts : a systematic review by the US Pharmacopeia. Drug safety : an international journal of medical toxicology and drug experience, 2008. 31(6): p. 46984.

131. Frank, J., et al., Daily consumption of an aqueous green tea extract supplement does not impair liver function or alter cardiovascular disease risk biomarkers in healthy men. The Journal of nutrition, 2009. 139(1): p. 58-62.

132. Bose, M., et al., The major green tea polyphenol, (-)-epigallocatechin-3-gallate, inhibits obesity, metabolic syndrome, and fatty liver disease in high-fat-fed mice. The Journal of nutrition, 2008. 138(9): p. 1677-83.

133. Bruno, R.S., et al., Green tea extract protects leptin-deficient, spontaneously obese mice from hepatic steatosis and injury. The Journal of nutrition, 2008. 138(2): p. 323-31.

134. Chen, Y.K., et al., Effects of green tea polyphenol (-)-epigallocatechin-3-gallate on newly developed high-fat/Western-style diet-induced obesity and metabolic syndrome in mice. Journal of agricultural and food chemistry, 2011.59(21): p. 11862-71.

135. Chung, M.Y., et al., Green tea extract protects against nonalcoholic steatohepatitis in ob/ob mice by decreasing oxidative and nitrative stress responses induced by proinflammatory enzymes. The Journal of nutritional biochemistry, 2012. 23(4): p. 361-7.

136. Nakamoto, K., et al., Beneficial Effects of Fermented Green Tea Extract in a Rat Model of Nonalcoholic Steatohepatitis. Journal of clinical biochemistry and nutrition, 2009. 44(3): p. 239-46.

137. Park, H.J., et al., Green tea extract attenuates hepatic steatosis by decreasing adipose lipogenesis and enhancing hepatic antioxidant defenses in ob/ob mice. The Journal of nutritional biochemistry, 2011. 22(4): p. 393-400.

138. Park, H.J., et al., Green tea extract suppresses NFkappaB activation and inflammatory responses in diet-induced obese rats with nonalcoholic steatohepatitis. The Journal of nutrition, 2012. 142(1): p. 57-63.

139. Ueno, T., et al., Epigallocatechin-3-gallate improves nonalcoholic steatohepatitis model mice expressing nuclear sterol regulatory element binding protein-1c in adipose tissue. International journal of molecular medicine, 2009. 24(1): p. 17-22.

140. Hertog, M.G., et al., Flavonoid intake and long-term risk of coronary heart disease and cancer in the seven countries study. Archives of internal medicine, 1995. 155(4): p. 381-6.

141. Russo, M., et al., The flavonoid quercetin in disease prevention and therapy: facts and fancies. Biochemical pharmacology, 2012. 83(1): p. 6-15.

142. Hollman, P.C., et al., Absorption of dietary quercetin glycosides and quercetin in healthy ileostomy volunteers. The American journal of clinical nutrition, 1995. 62(6): p. 1276-82.

143. Graefe, E.U., et al., Pharmacokinetics and bioavailability of quercetin glycosides in humans. Journal of clinical pharmacology, 2001. 41(5): p. 492-9.

144. Hollman, P.C., et al., Absorption and disposition kinetics of the dietary antioxidant quercetin in man. Free radical biology \& medicine, 1996. 21(5): p. 703-7.

145. Conquer, J.A., et al., Supplementation with quercetin markedly increases plasma quercetin concentration without effect on selected risk factors for heart disease in healthy subjects. The Journal of nutrition, 1998. 128(3): p. 593-7.

146. Harwood, M., et al., A critical review of the data related to the safety of quercetin and lack of evidence of in vivo toxicity, including lack of genotoxic/carcinogenic properties. Food and chemical toxicology : an international journal published for the British Industrial Biological Research Association, 2007. 45(11): p. 2179-205.

147. Boots, A.W., et al., The quercetin paradox. Toxicology and applied pharmacology, 2007. 222(1): p. 89-96. 


\section{Chapter 3}

148. Marcolin, E., et al., Quercetin treatment ameliorates inflammation and fibrosis in mice with nonalcoholic steatohepatitis. The Journal of nutrition, 2012. 142(10): p. 1821-8.

149. Marcolin, E., et al., Quercetin decreases liver damage in mice with non-alcoholic steatohepatitis. Basic \& clinical pharmacology \& toxicology, 2013. 112(6): p. 385-91.

150. Kobori, M., et al., Chronic dietary intake of quercetin alleviates hepatic fat accumulation associated with consumption of a Western-style diet in C57/BL6J mice. Molecular nutrition \& food research, 2011. 55(4): p. 530-40.

151. Jung, C.H., et al., Quercetin reduces high-fat diet-induced fat accumulation in the liver by regulating lipid metabolism genes. Phytotherapy research : PTR, 2013. 27(1): p. 139-43.

152. Boots, A.W., et al., Quercetin reduces markers of oxidative stress and inflammation in sarcoidosis. Clinical nutrition, 2011. 30(4): p. 506-12.

153. Edwards, R.L., et al., Quercetin reduces blood pressure in hypertensive subjects. The Journal of nutrition, 2007. 137(11): p. 2405-11.

154. Egert, S., et al., Quercetin reduces systolic blood pressure and plasma oxidised low-density lipoprotein concentrations in overweight subjects with a high-cardiovascular disease risk phenotype: a double-blinded, placebo-controlled cross-over study. The British journal of nutrition, 2009. 102(7): p. 1065-74.

155. Heinz, S.A., et al., Quercetin supplementation and upper respiratory tract infection: A randomized community clinical trial. Pharmacological research : the official journal of the Italian Pharmacological Society, 2010. 62(3): p. 237-42.

156. Valensi, P., et al., A multicenter, double-blind, safety study of QR-333 for the treatment of symptomatic diabetic peripheral neuropathy. A preliminary report. Journal of diabetes and its complications, 2005. 19(5): p. 247-53.

157. Gao, M., Y. Ma, and D. Liu, Rutin suppresses palmitic acids-triggered inflammation in macrophages and blocks high fat diet-induced obesity and fatty liver in mice. Pharmaceutical research, 2013. 30(11): p. 2940-50.

158. Hsu, C.L., et al., Phenolic compounds rutin and o-coumaric acid ameliorate obesity induced by highfat diet in rats. Journal of agricultural and food chemistry, 2009. 57(2): p. 425-31.

159. Ziaee, A., et al., Effects of rutin on lipid profile in hypercholesterolaemic rats. Basic \& clinical pharmacology \& toxicology, 2009. 104(3): p. 253-8.

160. Haenen, G.R., et al., Peroxynitrite scavenging by flavonoids. Biochemical and biophysical research communications, 1997. 236(3): p. 591-3.

161. van Acker, F.A., et al., Flavonoids can replace alpha-tocopherol as an antioxidant. FEBS letters, 2000. 473(2): p. 145-8.

162. Abou El Hassan, M.A., et al., The new cardioprotector Monohydroxyethylrutoside protects against doxorubicin-induced inflammatory effects in vitro. British journal of cancer, 2003. 89(2): p. 35762.

163. Mulvihill, E.E., et al., Naringenin decreases progression of atherosclerosis by improving dyslipidemia in high-fat-fed low-density lipoprotein receptor-null mice. Arteriosclerosis, thrombosis, and vascular biology, 2010. 30(4): p. 742-8.

164. Mulvihill, E.E., et al., Naringenin prevents dyslipidemia, apolipoprotein B overproduction, and hyperinsulinemia in LDL receptor-null mice with diet-induced insulin resistance. Diabetes, 2009. 58(10): p. 2198-210.

165. Zhu, W., et al., The anthocyanin cyanidin-3-O-beta-glucoside, a flavonoid, increases hepatic glutathione synthesis and protects hepatocytes against reactive oxygen species during hyperglycemia: Involvement of a cAMP-PKA-dependent signaling pathway. Free radical biology \& medicine, 2012.52(2): p. 314-27.

166. Guo, H., et al., Cyanidin 3-glucoside attenuates obesity-associated insulin resistance and hepatic steatosis in high-fat diet-fed and $\mathrm{db} / \mathrm{db}$ mice via the transcription factor FoxO1. The Journal of nutritional biochemistry, 2012. 23(4): p. 349-60.

167. Tsuda, T., et al., Dietary cyanidin 3-O-beta-D-glucoside-rich purple corn color prevents obesity and ameliorates hyperglycemia in mice. The Journal of nutrition, 2003. 133(7): p. 2125-30. 
168. Doddapattar, P., et al., Xanthohumol ameliorates atherosclerotic plaque formation, hypercholesterolemia, and hepatic steatosis in ApoE-deficient mice. Molecular nutrition \& food research, 2013.

169. Dorn, C., et al., Xanthohumol, a chalcon derived from hops, inhibits hepatic inflammation and fibrosis. Molecular nutrition \& food research, 2010. 54 Suppl 2: p. S205-13.

170. Guo, H.X., et al., Long-term baicalin administration ameliorates metabolic disorders and hepatic steatosis in rats given a high-fat diet. Acta pharmacologica Sinica, 2009. 30(11): p. 1505-12.

171. Lee, Y.S., et al., Nobiletin improves obesity and insulin resistance in high-fat diet-induced obese mice. The Journal of nutritional biochemistry, 2013. 24(1): p. 156-62.

172. Mei, L., M. Mochizuki, and N. Hasegawa, Hepatoprotective effects of pycnogenol in a rat model of non-alcoholic steatohepatitis. Phytotherapy research : PTR, 2012. 26(10): p. 1572-4.

173. Zheng, P., et al., Therapeutic effect of puerarin on non-alcoholic rat fatty liver by improving leptin signal transduction through JAK2/STAT3 pathways. The American journal of Chinese medicine, 2009. 37(1): p. 69-83.

174. Haenen, G.R., et al., Structure and activity in assessing antioxidant activity in vitro and in vivo A critical appraisal illustrated with the flavonoids. Environmental toxicology and pharmacology, 2006. 21(2): p. 191-8.

175. Wood, N., Hepatolipidemic effects of naringenin in high cornstarch-versus high coconut oil-fed rats. Journal of medicinal food, 2004. 7(3): p. 315-9. 



\section{Chapter 4}

\section{The role of SOD2 in the development of NASH}

Bregje van de Wier, Kristien J. Lemmens, Ger H. Koek, S.Eleonore Koehler, Eddie Wisse, Hans Duimel, Ronit Shiri-Sverdlov, Sofie M. A. Walenbergh, Aalt Bast, Guido R.M.M. Haenen 


\section{Abstract}

SOD2 is an important antioxidant enzyme in the mitochondria. Mitochondrial oxidative stress is believed to play an important role in the pathogenesis of nonalcoholic fatty liver disease (NAFLD). This study explores whether C57BL/6J mice with a liver-specific superoxide dismutase 2 (SOD2) knock-out develop non-alcoholic steatohepatitis (NASH) when fed a high fat high cholesterol diet (HFC diet).

Female SOD2 ${ }^{\mathrm{fl} / \mathrm{fl}} / \mathrm{Alb}-\mathrm{Cre} \mathrm{tg}^{\mathrm{tg}}$ mice with a liver-specific SOD2 knock-out (SOD2-KO mice), C57BL/6J wildtype mice (WT mice), and LDL receptor knock-out mice on a C57BL/6J background (Ldlr $\%$ mice) were fed a HFC diet for 3 weeks. After 3 weeks, the mice were sacrificed. Liver histology, electron microscopy, oxidative stress measurements and antioxidant gene expression analysis were performed.

The liver-specific SOD2 knock-out did not lead to an increase in oxidative stress in the livers of the studied mice. Also, no inflammation was found. In the Ldlr ${ }^{-/}$mice, an increased GSH/GSSG-ratio and increased inflammation was found compared to WT mice.

Unlike the Ldlr-/- mouse, the SOD2-KO ${ }_{\mathrm{L}}$ mouse on a HFC diet is not suited to study the onset of inflammation in NAFLD. The observation that SOD2 depletion in the liver in combination with a HFC diet is not enough to induce NASH, further supports the hypothesis that multiple hits are needed to develop NASH. 


\section{Introduction}

Non-alcoholic fatty liver disease (NAFLD) is the most common chronic liver disease in Western countries with an estimated prevalence of $20-30 \%[1,2]$. Its prevalence is expected to rise even more in line with that of obesity and diabetes [3]. A recent study in the United States found a prevalence of even 46\% in adult visitors of the Brooke Army Medical Centre [4]. NAFLD covers a spectrum of liver diseases that comprises steatosis, non-alcoholic steatohepatitis (NASH), fibrosis and cirrhosis. In the past, the pathogenesis of the disease was modeled according to the two hit theory [5]. In this concept steatosis was the first hit followed by a second hit inducing inflammation. This idea has now been abandoned. NAFLD is currently regarded as a multiple parallel hits disease [6]: multiple hits involving metabolic changes, oxidative stress and proinflammatory mechanisms induce inflammation and fibrosis in a genetically susceptible person.

Although many studies have been conducted, there is no effective treatment for NAFLD yet. Animal models are often used to investigate potential drugs. Various models have been developed, mostly genetic or dietary models or a combination of both [7-10]. However, due to the multifaceted nature of NAFLD, no animal model is available up to date that comprises all of the various facets of NAFLD [11]. Because the animal model that perfectly reflects NAFLD is lacking, the animal model for a study should be selected based on the specific aspect of the disease that is studied.

The facet we aim to study is the onset of inflammation in NAFLD induced by an increase in oxidative stress. We investigated whether the $\mathrm{SOD} 2-\mathrm{KO}_{\mathrm{L}}$ mouse develops NASH when fed a HFC diet. Because mitochondrial oxidative stress is believed to have a key role in the pathogenesis of NASH, we hypothesized that knock-out of SOD2, which scavenges superoxide radicals in the mitochondria, will dramatically increase oxidative stress with subsequent induction of inflammation. WT mice, that only develop steatosis when fed a high fat diet for three weeks [12], and Ldlr ${ }^{-/}$mice, that develop steatohepatitis within three weeks of HFC diet intake [13], were used for comparison. 


\section{Materials and methods}

\section{Animals and treatment}

Female WT mice, female $\mathrm{Ldll}^{-/}$- mice and female SOD2- $\mathrm{KO}_{\mathrm{L}}$ mice were obtained from Jackson laboratories or bred at the breeding facility of Maastricht University. SOD2 flox mice were produced by Shirasawa et al. [14] and kindly provided by dr. Thomas Kietzmann, University of Kaiserlautern, Germany. Mice were kept in a temperatureand light-controlled facility and were permitted ad libitum consumption of water and chow. At the age of 13 weeks, all mice were subjected to a HFC diet (D11012302) (Research Diets, New Brunswick, USA) with $0.2 \%$ cholesterol and fat derived from palm oil. Mice were divided into three experimental groups according to their background: $\mathrm{WT}, \mathrm{Ldlr}^{-/}$- mice and SOD2-KO $\mathrm{L}(\mathrm{n}=12$ for each group). All mice received daily subcutaneous injections of a physiological saline solution $(25 \mu \mathrm{l}$ per $\mathrm{g}$ of body weight of $0.9 \mathrm{mg} \mathrm{NaCl} / \mathrm{mL}$ sterile water), in order to serve as control groups for a simultaneous intervention study [15].

Mice were weighed every third day and food intake was recorded per two mice housed together. Diet and treatment were continued for three weeks, after which mice were anaesthetized with $0.05 \mathrm{mg} / \mathrm{kg}$ buprenorphine and isoflurane $0.4 \mathrm{l} / \mathrm{min}$ and sacrificed by blooding via the $v$. cava. The liver was removed and divided for further investigation. One part of the large left lobe was used for RNA isolation, another part to prepare frozen liver sections and the final part to prepare formalin-fixed paraffinembedded liver sections. One part of the right lobe was used for electron microscopy. The remainder of the liver was used to perform oxidative stress measurements.

\section{Histology}

Formalin-fixed, paraffin-embedded sections $(4 \mu \mathrm{m})$ of hepatic tissue were stained with hematoxylin-eosin using a standard protocol. Livers were evaluated using the NAFLD activity score (NAS) [16] by researchers who were blinded to the genotype of the experimental animals. Steatosis grade was determined to be 0 for $<5 \%$ steatosis, 1 for $5-33 \%$ steatosis, 2 for $33-66 \%$ steatosis and 3 for $>66 \%$ steatosis. Foci of lobular inflammation were counted in four $200 x$ fields. If no inflammatory foci were present, inflammation was graded as $0,<2$ foci/field were graded as $1,2-4$ foci as 2 and $>4$ foci as 3 . 
Frozen liver sections $(7 \mu \mathrm{M})$ were stained for infiltrated macrophages (macrophage marker: MAC-1) and neutrophils (neutrophil marker: NIMP). Stained cells were counted by two independent investigators, using six photographs of each liver section. Photographs were taken using a Nikon DMX1200 digital camera and ACT-1 version 2.63 software.

\section{Electron microscopy}

Liver tissue samples were fixed by perfusion-fixation using $3 \%$ glutaraldehyde in 0.09 $\mathrm{M}$ potassium phosphate $+1.4 \%$ sucrose at room temperature. Samples were preserved in this solution at $4{ }^{\circ} \mathrm{C}$ until further processing. Samples were washed with $0.09 \mathrm{M}$ potassium phosphate $+7.5 \%$ sucrose at room temperature to avoid chemical reactions between glutaraldehyde and osmium. After that they were post fixed with $1 \% \mathrm{OsO}_{4}$ in Veronal Buffer $+1.5 \%$ ferrocyanide $(\mathrm{pH} 7.4)$ at $4^{\circ} \mathrm{C}$ for 1 hour. They were rinsed in Veronal buffer $+7 \%$ sucrose at $4{ }^{\circ} \mathrm{C}$ for 5 minutes. All samples were dehydrated in a series of $70 \%, 90 \%$ and $2 \times 100 \%$ alcohol. Samples were incubated for $2 \times 30$ minutes in propylene oxide at room temperature. After this step they were kept in Epon embedding medium with propylene oxide (1:1) overnight. The next day they were embedded in a BEEM ${ }^{\circledR}$ capsule and polymerised with fresh Epon for 72 hours at - $50^{\circ} \mathrm{C}$. After fixation samples were cut into $1 \mu \mathrm{m}$ sections, using a microtome. Sections were aligned under a light microscope to prepare them for transmission electron microscopy (TEM). Eighty nm sections for TEM were prepared with a diamond knife and an ultramicrotome. Slides were placed on a 75 mesh copper grid and stained with uranyl acetate and lead citrate before placing them in the transmission electron microscope (Philips CM100). Several images were taken of each tissue sample.

\section{Oxidative stress measurements}

Liver homogenate $(250 \mathrm{mg}$ liver/ml) was prepared in $145 \mathrm{mM}$ potassium phosphate buffer ( $\mathrm{pH}$ 7.4). Malondialdehyde (MDA) was used as a measure of lipid peroxidation. MDA was determined by complexation of MDA with thiobarbituric acid $[17,18]$ and quantified by HPLC as described by Boots et al. [19], using liver homogenate instead of plasma .Trolox equivalent antioxidant capacity (TEAC), which represents the total antioxidant capacity of the liver, was measured according to Fischer et al. [20]. Absorption was measured at $734 \mathrm{~nm}$ and $37{ }^{\circ} \mathrm{C}$ with a Varian Cary 50 spectrophotometer. Total glutathione (GSH) content and glutathione disulfide (GSSG) were determined by the enzymatic recycle method described by Rahman et al. [21] 
using the conversion of 5,5'-dithio-bis(2-nitrobenzoic acid) (DTNB) into $5^{\prime}$-thio-2nitrobenzoic acid (TNB). The formation of TNB was measured spectrophotometrically at $412 \mathrm{~nm}$ and $37^{\circ} \mathrm{C}$. The GSH/GSSG-ratio was calculated as a measure of antioxidant protection.

\section{RNA isolation and real-time PCR}

Liver parts that were stored in RNA later $\AA$ at $-80^{\circ} \mathrm{C}$ were used for RNA isolation using miRNeasy Mini Kit with DNase treatment (Qiagen, Venlo, the Netherlands) according to manufacturer's instructions. Isolated RNA was converted to complementary DNA (cDNA) using the iScript cDNA synthesis kit (Bio-rad, Veenendaal, the Netherlands). Real-time PCR was performed with a BioRad MyiQ iCycler Single Color RT-PCR detection system using Sensimix ${ }^{\mathrm{TM}}$ Plus SYBR and Fluorescein (Quantace-Bioline, Alphen a/d Rijn, The Netherlands), five $\mu$ d diluted (10x) cDNA, and $0.3 \mu \mathrm{M}$ primers in a total volume of $25 \mu \mathrm{l}$. PCR was performed as follows: denaturation at $95^{\circ} \mathrm{C}$ for 10 minutes, followed by 40 cycles at $95^{\circ} \mathrm{C}$ for 15 seconds and at $60^{\circ} \mathrm{C}$ for 45 seconds. After PCR a melting curve $\left(60-95^{\circ} \mathrm{C}\right)$ was produced to identify the product and assess its purity. Gapdh (glyceraldehyde-3-phosphate dehydrogenase) and Ywhaz (tyrosine 3-monooxygenase/tryptophan 5-monooxygenase activation protein, zeta polypeptide) were used as housekeeping genes [22]. There was a good correlation between the $\Delta$ Ct-values of Gapdh and Ywhaz $\left(\mathrm{R}^{2}=0.8041\right)$. To rule out an effect of small differences in housekeeping genes on the outcome of measurements, the average value of Gapdh and Ywhaz was used for calculation of the $\Delta \mathrm{Ct}$-value. Primer sequences are listed in table 1. Data were analysed using the MyIQ software system (BioRad) and were expressed as relative gene expression (fold change) using the $2^{\Delta \Delta C t}$ method. 
Table 1. Primer sequences

\begin{tabular}{lll}
\hline Gene & Forward primer & Reverse primer \\
\hline Gclc & CTGCACATCTACCACGCAGT & TTCATGATCGAAGGACACCA \\
\hline Hmox1 & GAGCCTGAATCGAGCAGAAC & CCTTCAAGGCCTCAGACAAA \\
\hline Nqo1 & CCAATCAGCGTTCGGTATTA & GTCTTCTCTGAATGGGCCAG \\
\hline Gpx1 & GTTTCCCGTGCAATCAGTTC & CAATGTAAAATTGGGCTCGAA \\
\hline Gpx2 & GGTAGTTCTCGGCTTCCCTT & AGGTCGGACATACTTGAGGC \\
\hline Gpx4 & CATCCTGCCTTCTGTCCCT & ATGGTACCACTCATACCGCC \\
\hline Cat & CCGTCTGAGCCGCTTACTTA & GCTGAGAATTCGTGCATGG \\
Sod1 & GATGAAGCAGTGGAAGGAGC & CCCGCGGTCATGATATTAAGT \\
\hline Sod2 & GAACCATCCACTTCGAGCA & TACTGATGGACGTGGAACCC \\
\hline Sod3 & ACTGAAGTTCAATGGTGGGG & GCTTGATAGCCTCCAGCAAC \\
\hline Gapdh & ACTCAGAGGCTCTTCCTCCG & ATGGCTGAGGTTCTCTGCAC \\
Ywhaz & TTGATGGCAACAATCTCCAC & CGTCCCGTAGACAAAATGGT \\
\hline
\end{tabular}

\section{Statistics}

Data were analysed with GraphPad Prism 5. Data presented in the study are expressed as mean \pm standard deviation (SD). Differences between groups were analyzed using the Kruskal-Wallis test. Post hoc testing was performed using the Mann-Whitney Utest with Bonferroni correction. A P-value less than 0.05 was considered to be statistically significant, a P-value between 0.05 and 0.1 was considered as indicating a trend.

\section{Results}

\section{Body weight and food intake}

There were no significant differences in food intake between groups (table 2). Initial and final body weight and liver weight also did not differ between groups. Liver/total body weight-ratio tended to be increased in Ldlr $/-$ mice and SOD2- $\mathrm{KO}_{\mathrm{L}}$ mice compared to WT mice $(\mathrm{P}=0.09)$. However, this did not reach significance. 
Table 2: Characteristics

\begin{tabular}{llll}
\hline & WT & SOD2-KO & Ldlr $^{-{ }^{-}}$ \\
\hline Initial body weight $(g)$ & $20.8 \pm 1.3$ & $20.1 \pm 2.1$ & $20.9 \pm 1.1$ \\
\hline Final body weight $(\mathbf{g})$ & $21.3 \pm 1.5$ & $20.4 \pm 2.0$ & $21.3 \pm 1.5$ \\
\hline Body weight change $(\mathrm{g})$ & $0.41 \pm 0.71$ & $0.34 \pm 0.84$ & $0.38 \pm 1.17$ \\
\hline Liver weight (g) & $1.00 \pm 0.14$ & $1.00 \pm 0.12$ & $1.05 \pm 0.12$ \\
\hline Liver/total body weight-ratio (\%) & $4.69 \pm 0.40$ & $4.88 \pm 0.45$ & $4.95 \pm 0.35$ \\
\hline Average food intake (g/day/cage) & $4.02 \pm 0.73$ & $4.15 \pm 0.71$ & $4.33 \pm 0.73$ \\
\hline
\end{tabular}

Initial and final body weight, body weight change during the study, liver weight, liver/total body weight and average food intake per two mice during the study. Mean $\pm S D, n=12$ for each group.

\section{Histology}

No difference in degree of steatosis (steatosis score) was found between the groups (figure 1A). In SOD2-KO $\mathrm{K}_{\mathrm{L}}$ mice, there was no significant difference in the amount of inflammatory foci per microscopic field (inflammation score) compared to WT mice (figure 1B). In Ldlr/- mice, the amount of inflammatory foci was significantly higher than the amount of inflammatory foci in WT mice and SOD2- $\mathrm{KO}_{\mathrm{L}}$ mice (figure $1 \mathrm{~B}$ ). In the livers of $\mathrm{Ldlr}^{-}$- mice an increased amount of infiltrated macrophages and neutrophils were found compared to wildtype mice and $\mathrm{SOD} 2-\mathrm{KO}_{\mathrm{L}}$ mice (figure $2 \mathrm{~A}$ and $\mathrm{B}$ ). This was not observed in the $\mathrm{SOD} 2-\mathrm{KO}_{\mathrm{L}}$ mice (figure $2 \mathrm{~A}$ and $\mathrm{B}$ ). 

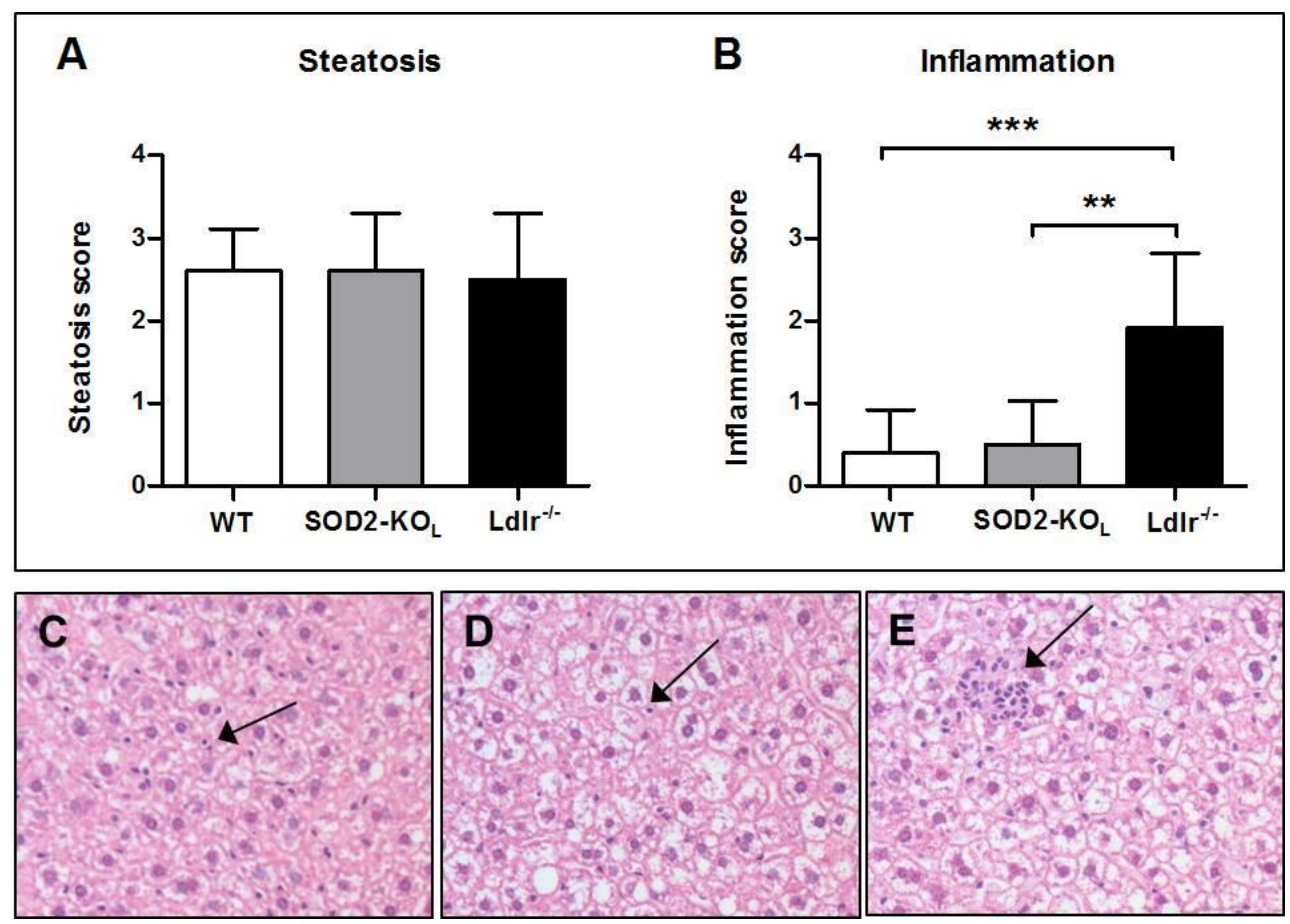

Figure 1. Liver histology. Steatosis grade (panel A) and lobular inflammation (panel B) were appraised on a scale of 0 to 3 according to the NAS-score ( $\mathrm{n}=10$ for WT, $\mathrm{n}=10$ for SOD2-KOL, $\mathrm{n}=12$ for Ldlr ${ }^{-}$, not all formalin-fixed sections were suitable for these quantifications) ${ }^{*} \mathrm{p}=0.01-0.05,{ }^{* *} \mathrm{p}=0.001-0.01,{ }^{* * *} \mathrm{p}<$ 0.001. Typical examples of livers from C) a WT mouse with a few individual inflammatory cells (indicated by the arrow), but no inflammatory foci, D) a SOD2-KOL mouse with a few individual inflammatory cells (indicated by the arrow), but no inflammatory foci and E) a Ldlr $\%$ mouse with inflammatory foci (indicated by the arrow), magnification 200x. 
A

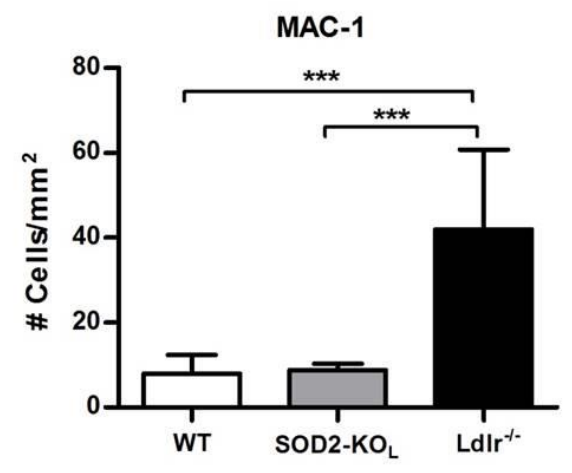

B

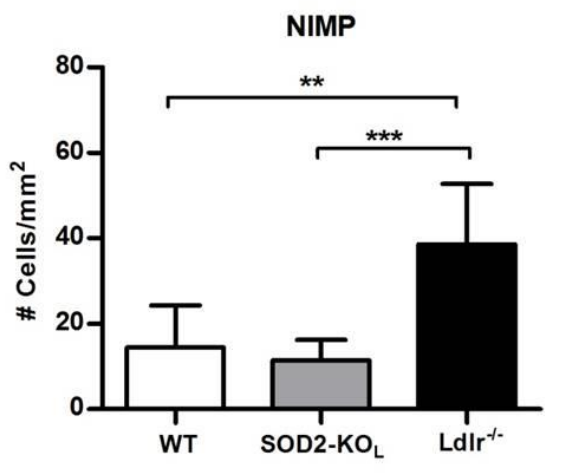

Figure 2. MAC-1 and NIMP staining. A) MAC-1 staining for infiltrated macrophages ( $\mathrm{n}=12$ for $\mathrm{WT}$ mice, $\mathrm{n}=$ 11 for SOD2 $-\mathrm{KO}_{\mathrm{L}}$ mice and $\mathrm{n}=10$ for Ldlr ${ }^{-}$mice) ${ }^{* * *} \mathrm{p}<0.001$. B) NIMP staining for neutrophils $(\mathrm{n}=12$ for WT mice, SOD2-KO $\mathrm{L}_{\mathrm{L}}$ mice and Ldlr $\%$ mice) ${ }^{* *} \mathrm{p}=0.001-0.01,{ }^{* * *} \mathrm{p}<0.001$.

\section{Electron microscopy}

Macrovesicular as well as microvesicular steatosis was seen in all mice. In Ldlr $\%$ mice, fat accumulation was seen in Kupffer cells. This was not observed in wildtype mice and SOD2- $\mathrm{KO}_{\mathrm{L}}$ mice. Glycogen deposits were found in the hepatocytes of all groups. Minor signs of inflammation were observed in all groups, but were difficult to quantify with electron microscopy. Apoptosis was not observed. In several livers, necrotic hepatocytes were observed. Apart from the fat accumulation in Kupffer cells, no significant differences were found between the different groups using electron microscopy.

\section{Oxidative stress measurements}

No significant differences between groups were found for TEAC and MDA measurements (figure 3A and B). The GSH/GSSG-ratio in Ldlr/- mice was significantly higher than the GSH/GSSG-ratio in wildtype and SOD2-KO $\mathrm{K}_{\mathrm{L}}$ mice, indicating increased antioxidant protection in Ldlr/- mice (figure 3C). 


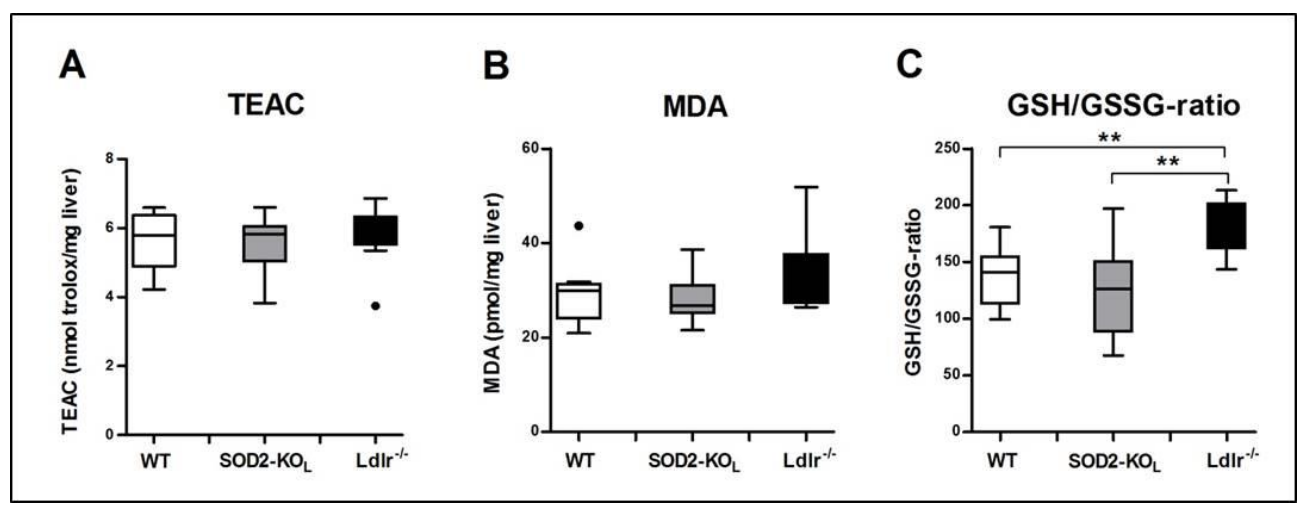

Figure 3. TEAC, MDA and GSH/GSSG-ratio. A) Measurement of Trolox equivalent antioxidant capacity (TEAC), representing the total antioxidant capacity of the liver ( $\mathrm{n}=12$ for WT mice, SOD2-KO $\mathrm{L}$ mice and Ldlr $\%$ mice). B) Determination of malondialdehyde (MDA) as a measure of lipid peroxidation ( $\mathrm{n}=12 \mathrm{for}$ WT mice, SOD2- $\mathrm{KO}_{\mathrm{L}}$ mice and Ldlr $\%$ mice). C) Calculated GSH/GSSG-ratio as a measure of antioxidant protection ( $\mathrm{n}=12$ for WT mice, SOD2-KOL mice and Ldlr $\%$ mice ${ }^{* *} \mathrm{p}=0.001-0.01$.

\section{Antioxidant gene expression}

As expected, Sod2 gene expression was significantly lower in the livers of SOD2- $\mathrm{KO}_{\mathrm{L}}$ mice, compared to WT and Ldlr $\%$ mice. Hmox1 and Gpx3 gene expression were significantly higher in Ldlr $/$ mice compared to WT mice (table 3). Catalase gene expression was significantly lower in Ldlr $\%$ mice compared to WT mice (table 3). Gpx1 gene expression was significantly lower in $\mathrm{Ldll}^{-} /$mice compared to SOD2- $\mathrm{KO}_{\mathrm{L}}$ mice and tended to be lower compared to WT mice, but this did not reach significance $(\mathrm{P}=0.09)$ (table 3). No differences in gene expression were found between groups for Gpx2, Gpx4, Nqo1, Gclc, Sod1, Sod3 and Cyp2e1 (table 3). 
Table 3: Fold change in gene expression compared to wildtype mice

\begin{tabular}{llll}
\hline Gene & WT & SOD2-KO $_{\text {L }}$ & Ldlr $^{-1-}$ \\
\hline Hmox1 & $1.00 \pm 0.40$ & $1.30 \pm 0.45$ & $2.27 \pm 1.48^{\mathrm{a}}$ \\
\hline Nqo1 & $1.00 \pm 0.51$ & $1.47 \pm 1.58$ & $0.82 \pm 0.25$ \\
Gclc & $1.00 \pm 0.43$ & $1.35 \pm 1.20$ & $1.40 \pm 1.14$ \\
\hline Gpx1 & $1.00 \pm 0.32$ & $1.16 \pm 0.48$ & $0.74 \pm 0.24^{\mathrm{b}}$ \\
Gpx2 & $1.00 \pm 0.55$ & $1.09 \pm 0.34$ & $1.11 \pm 0.23$ \\
\hline Gpx3 & $1.00 \pm 0.57$ & $1.35 \pm 0.56$ & $2.21 \pm 1.01^{\mathrm{a}}$ \\
Gpx4 & $1.00 \pm 0.43$ & $0.97 \pm 0.32$ & $0.75 \pm 0.32$ \\
Sod1 & $1.00 \pm 0.58$ & $1.04 \pm 0.54$ & $1.04 \pm 0.51$ \\
\hline Sod2 & $1.00 \pm 0.41$ & $0.05 \pm 0.02^{\mathrm{a}, \mathrm{c}}$ & $0.99 \pm 0.43$ \\
Sod3 & $1.00 \pm 0.47$ & $1.31 \pm 0.90$ & $1.13 \pm 0.39$ \\
Cat & $1.00 \pm 0.41$ & $0.82 \pm 0.55$ & $0.71 \pm 0.18^{\mathrm{a}}$ \\
Cyp2e1 & $1.00 \pm 0.52$ & $1.01 \pm 0.54$ & $0.75 \pm 0.36$ \\
\hline
\end{tabular}

$a=$ significantly different compared to WT mice, $b=$ significantly different compared to $\mathrm{SOD} 2-\mathrm{KO}_{\mathrm{L}}$ mice, $c=$ significantly different compared to $\mathrm{Ldl} /$ mice.

\section{Discussion}

Many animal models have been developed to investigate NAFLD and NASH [7-10]. Because NAFLD is such a multifactorial disease, with multiple pathways leading to the onset of inflammation and further liver damage, it is difficult to incorporate all the facets of the disease in one animal model [11]. Ideally, an animal model should display liver damage resembling human NAFLD (steatosis, inflammation and fibrosis), involving the most important pathogenic pathways (metabolic abnormalities, oxidative stress and inflammation) and express other signs of the metabolic syndrome (dyslipidemia, insulin resistance, obesity) [11]. Although some animal models approach the human etiopathology, the perfect animal model of NAFLD has not been developed. Therefore, the choice of animal model could best be based on the facet or characteristic of NAFLD that is investigated in that study.

Our study examined whether a liver-specific SOD2 knock-out mouse on a HFC diet is a suitable model to investigate the onset of inflammation in NAFLD, involving oxidative stress. We compared this model with wildtype mice on a HFC diet. Wildtype mice are known to develop steatosis within a few weeks when consuming a high fat diet, but they only develop inflammation after long-term consumption of a high fat diet [12]. Furthermore, we compared the SOD2-KO $\mathrm{L}_{\mathrm{L}}$ mouse with the Ldlr-/- mouse on a HFC diet, a model that has been used to investigate inflammation in NAFLD [13]. 
SOD catalyzes the dismutation of superoxide to hydrogen peroxide and oxygen. SOD1 or copper/zinc superoxide dismutase is located predominantly in the cytoplasm. SOD2 or manganese superoxide dismutase is located in the mitochondria and is the primary defense against mitochondrial superoxide. Other antioxidants that are present in eukaryotic cells, like GSH, ascorbate and tocopherols do not inactivate superoxide as rapidly or effectively as SOD [23].

Because SOD2 is located in the mitochondria, one of the major sources of reactive oxygen species, SOD2 knock-out is expected to severely increase oxidative stress. Previous studies have proven that SOD2 knock-out has detrimental effects. Lines of homozygous SOD2 knock-out mice die within a few weeks after birth. They exhibit severe anemia, degeneration of neurons in the basal ganglia and brainstem, progressive motor disturbances, dilated cardiomyopathy and fatty liver [23, 24]. However, heterozygous SOD2 knock-out mice, displaying a reduction in SOD2 activity of about $50 \%$ in various tissues, present only with mild oxidative stress in the mitochondria and do not show any clinical significant changes [24, 25].

We hypothesized that complete ablation of SOD2 in the liver will dramatically increase oxidative stress in the liver. We expected that in combination with a HFC, this might induce NASH. Remarkably, we did not find induction of inflammation in SOD2- $\mathrm{KO}_{\mathrm{L}}$ mice given a HFC diet. Also, oxidative stress parameters and genes were not altered compared to wildtype mice. Our results are in line with those of Garcia Caraballo et al [26]. They demonstrated that heterozygous, hepatocyte-specific, SOD2-deficient mice given a HFC diet do not develop inflammation and oxidative damage in the liver. Although a higher level of oxidative stress in the liver is expected in our model, due to the complete ablation of SOD2, none of the measured biomarkers revealed that oxidative stress increased. Interestingly, no evidence for upregulation of other antioxidants was found in the SOD2- $\mathrm{KO}_{\mathrm{L}}$ mice, since the expression of antioxidants genes that were measured was not increased.

An explanation for our findings could be that formation of superoxide in other locations than the mitochondria is more important in the development of inflammation in NAFLD. The generation of superoxide by CYP2E1, which is predominantly located in the endoplasmic reticulum, could be a candidate. Superoxide produced in the endoplasmic reticulum will be most likely scavenged by SOD 1, which is located in the cytoplasm. An additional explanation might be that reactive oxygen species that are not scavenged by SOD, such as the hydroxyl radical and hydrogen peroxide, are more important in the development of inflammation in NAFLD than 
superoxide. Furthermore, in a previous study of Krautbauer et al. [27], it was observed that SOD2 expression is decreased in male patients and rodents with NAFLD, but not in female patients and rodents with NAFLD. They suggest that low SOD2 levels might contribute to oxidative stress and disease progression in males, but not in females and that the expression of SOD2 might be affected by sex hormones. Since we only used female mice in our study, this might also have contributed. Finally, it is likely that knock-out of SOD2 in the liver in combination with a HFC diet is simply not sufficient to induce NASH, because additional hits are needed in the development of this multifactorial disease.

In conclusion, our study demonstrates that the female SOD2- $\mathrm{KO}_{\mathrm{L}}$ mouse is not a good model to investigate the onset of inflammation in NAFLD. Surprisingly, SOD2 knockout in the liver does not increase oxidative stress and does not subsequently induce inflammation in these mice. The observation that SOD2 depletion in combination with a HFC diet is not enough to induce inflammation, further supports the hypothesis that multiple hits are needed in the development of NASH.

\section{Acknowledgement}

The authors would like to thank Kelly Waagmeester, Leonie Jonkers, Marie-José Drittij, Wout Lamers and Paul van Dijk for their help with the experiments. 


\section{References}

1. Bedogni, G., et al., Prevalence of and risk factors for nonalcoholic fatty liver disease: the Dionysos nutrition and liver study. Hepatology, 2005. 42(1): p. 44-52.

2. Browning, J.D., et al., Prevalence of hepatic steatosis in an urban population in the United States: impact of ethnicity. Hepatology, 2004. 40(6): p. 1387-95.

3. Bjornsson, E. and P. Angulo, Non-alcoholic fatty liver disease. Scand J Gastroenterol, 2007. 42(9): p. 1023-30.

4. Williams, C.D., et al., Prevalence of nonalcoholic fatty liver disease and nonalcoholic steatohepatitis among a largely middle-aged population utilizing ultrasound and liver biopsy: a prospective study. Gastroenterology, 2011. 140(1): p. 124-31.

5. Day, C.P. and O.F.W. James, Steatohepatitis: A Tale of Two "Hits"? Gastroenterology, 1998. 114(4): p. 842-845.

6. Tilg, H. and A.R. Moschen, Evolution of inflammation in nonalcoholic fatty liver disease: the multiple parallel hits hypothesis. Hepatology, 2010. 52(5): p. 1836-46.

7. Nagarajan, P., et al., Genetically modified mouse models for the study of nonalcoholic fatty liver disease. World J Gastroenterol, 2012. 18(11): p. 1141-53.

8. Nanji, A.A., Animal models of nonalcoholic fatty liver disease and steatohepatitis. Clin Liver Dis, 2004. 8(3): p. 559-74, ix.

9. Takahashi, Y., Y. Soejima, and T. Fukusato, Animal models of nonalcoholic fatty liver disease/nonalcoholic steatohepatitis. World J Gastroenterol, 2012. 18(19): p. 2300-8.

10. Schattenberg, J.M. and P.R. Galle, Animal models of non-alcoholic steatohepatitis: of mice and man. Dig Dis, 2010. 28(1): p. 247-254.

11. Van de Wier, B., et al., The potential of flavonoids in the treatment of non-alcoholic fatty liver disease. Crit Rev Food Sci Nutr, 2015. [Epub ahead of print].

12. Ito, M., et al., Longitudinal analysis of murine steatohepatitis model induced by chronic exposure to high-fat diet. Hepatol Res, 2007. 37(1): p. 50-7.

13. Bieghs, V., et al., LDL receptor knock-out mice are a physiological model particularly vulnerable to study the onset of inflammation in non-alcoholic fatty liver disease. PLoS One, 2012. 7(1): p. e30668.

14. Ikegami, T., et al., Model mice for tissue-specific deletion of the manganese superoxide dismutase (MnSOD) gene. Biochem Biophys Res Commun, 2002. 296(3): p. 729-36.

15. Lemmens, K.J., et al., The flavonoid monoHER promotes the adaption to oxidative stress during the onset of NAFLD. Biochem Biophys Res Commun, 2015. 456(1): p. 179-82.

16. Kleiner, D.E., et al., Design and validation of a histological scoring system for nonalcoholic fatty liver disease. Hepatology, 2005. 41(6): p. 1313-21.

17. Haenen, G.R.M.M. and A. Bast, Protection against lipid peroxidation by a microsomal glutathionedependent labile factor. FEBS letters, 1983. 159(1-2): p. 24-28.

18. Lepage, G., et al., Preparative steps necessary for the accurate measurement of malondialdehyde by high-performance liquid chromatography. Anal Biochem, 1991. 197(2): p. 277-83.

19. Boots, A.W., et al., Quercetin reduces markers of oxidative stress and inflammation in sarcoidosis. Clin Nutr, 2011. 30(4): p. 506-12.

20. Fischer, M.A., et al., Determination of the antioxidant capacity in blood. Clinical chemistry and laboratory medicine : CCLM / FESCC, 2005. 43(7): p. 735-40.

21. Rahman, I., A. Kode, and S.K. Biswas, Assay for quantitative determination of glutathione and glutathione disulfide levels using enzymatic recycling method. Nature protocols, 2006. 1(6): p. 3159-65.

22. Bruce, K.D., et al., The housekeeping gene YWHAZ remains stable in a model of developmentally primed non-alcoholic fatty liver disease. Liver International, 2012. 32(8): p. 1315-1321.

23. Lebovitz, R.M., et al., Neurodegeneration, myocardial injury, and perinatal death in mitochondrial superoxide dismutase-deficient mice. Proc Natl Acad Sci U S A, 1996. 93(18): p. 9782-7. 


\section{Chapter 4}

24. Li, Y., et al., Dilated cardiomyopathy and neonatal lethality in mutant mice lacking manganese superoxide dismutase. Nat Genet, 1995. 11(4): p. 376-81.

25. Lee, Y.H., et al., Proteomics profiling of hepatic mitochondria in heterozygous Sod2+/- mice, an animal model of discreet mitochondrial oxidative stress. Proteomics, 2008. 8(3): p. 555-68.

26. Garcia Caraballo, S.C., et al., Reduced expression of hepatic superoxide dismutase 2 in mice does not affect the antisteatotic response to high-protein and high-fat diets. Submitted to Nutrition \& Metabolism, 2015.

27. Krautbauer, S., et al., Manganese superoxide dismutase is reduced in the liver of male but not female humans and rodents with non-alcoholic fatty liver disease. Exp Mol Pathol, 2013. 95(3): p. 330-5. 


\section{Chapter 5}

\section{The flavonoid MonoHER promotes the adaptation to oxidative stress during the onset of NAFLD}

Kristien J. A. Lemmens*, Bregje van de Wier*, Ger H. Koek, S. Eleonore Köhler, Marie-José Drittij, Wim J.F. van der Vijgh, Aalt Bast, Guido R.M.M. Haenen

Biochemical and Biophysical Research Communications. 2015; 456(1): 179-82

* equal contribution 


\section{Abstract}

Non-alcoholic fatty liver disease (NAFLD) is the most common liver disease. An evidence-based pharmacological treatment for NAFLD is still lacking, but flavonoids have shown therapeutic potential. The present study was designed to investigate the effect of the flavonoid monoHER on the onset of NAFLD in Ldlr/- mice on a high-fat and high-cholesterol diet. The focus was put on the effect on oxidative stress as well as the adaptive response. Wild type mice served as a control and the effect of monoHER was compared to that of a placebo.

In the $\mathrm{Ldlr} /$ - group, monoHER provided only a mild protection against oxidative stress. In the placebo $L d l r /$ group an adaptive response elicited by the NRF2 antioxidant defence system was observed, evidenced by a higher Hmox1 and Gpx3 gene expression, as well as an increased redox status, evidenced by the higher GSH/GSSG ratio. In the monoHER treated $\mathrm{Ldll}^{\mathrm{K}}$ - group both the adaptive response as well as the increase in redox status tended to be higher, although this did not reach significance on a group level. Unexpectedly, a strong within animal relationship was found that links a high adaptive response to a low redox status in the monoHER Ldlr-/- group. This correlation was absent in the placebo and wildtype group.

The concept that emerges is that a thiol-reactive oxidation product of monoHER, formed during oxidative stress, selectively induces the NRF2 pathway and enforces the endogenous antioxidant shield, to provide protection against NAFLD. 


\section{Introduction}

Non-alcoholic fatty liver disease (NAFLD) is the most common liver disease in Western countries affecting $20-30 \%$ of the general population, and this percentage is still on the rise. NAFLD includes a spectrum of liver disorders ranging from steatosis to non-alcoholic steatohepatitis (NASH), fibrosis, cirrhosis and hepatocellular carcinoma [1]. Up to date, no evidence-based pharmacotherapy is available for NAFLD. To find a way to prevent NAFLD, the molecular processes involved in the onset of the disease should be controlled.

One of the hallmarks in the etiology of NAFLD is oxidative stress [2, 3]. During oxidative stress, the unbalanced production of reactive oxygen species (ROS) results in damage to virtually any cellular component, which explains the prominent role of ROS in NAFLD. However, ROS also destruct Kelch-like ECH-associated protein 1 (KEAP1), the inhibitor protein of NRF2. NRF2 serves as a master redox switch which turns on the expression of endogenous antioxidant genes e.g. heme oxygenase 1 (Hmox1) and glutathione peroxidase 3 (Gpx3) [4] and ultimately increases the cellular redox potential. Consequently, cells adapt to ROS through this potent feedback mechanism which enforces the protection against ROS. This fits in the current concept that health is a dynamic process characterized by the ability to adapt to challenges on a delicate homeostasis [5].

In this concept of health, drugs are bioactive compounds that help to restore and enforce homeostasis. Numerous studies have demonstrated the beneficial effect of flavonoids - a group of antioxidant bioactives commonly found in our diet - in animal models of NAFLD [6]. Among the flavonoids, 7-mono-0-( $\beta$-hydroxyethyl)-rutoside (monoHER) appears to be one of the most promising ones for treatment of NAFLD [6]. This flavonoid is the main ingredient of Venoruton, a drug that has been used for several decades in the treatment of venous insufficiency. MonoHER displays excellent antioxidant activity $[7,8]$, has a relatively high bioavailability and is relatively safe [6]. Based on these favorable characteristics monoHER was selected for this study.

To evaluate its efficacy, the effect of monoHER on oxidative stress and the adaptive response during the onset of NAFLD was determined in an animal model. Previous studies have shown that Ldlr $/$ mice on a high-fat and high-cholesterol diet are a suitable model to study the development of NAFLD [9]. Wild type mice served as control and the effect of monoHER was compared to that of a placebo. The effect was 
evaluated on three levels, namely the direct damage by ROS, the NRF2-induced gene expression and the adaptive response on the cellular redox status.

\section{Material and methods}

\section{Animals and treatment}

Female C57BL/6J wildtype mice (WT mice) were obtained from Jackson laboratories (Bar Harbor, Maine, USA) and bred at the breeding facility of Maastricht University. Female LDL-receptor knockout mice on a C57BL/6J background ( $\mathrm{Ldlr} /$ - mice) were bred at the breeding facility of Maastricht University. Mice were maintained in a temperature- and light-controlled facility and were permitted ad libitum consumption of water and chow. At the age of 13 weeks, all mice started a high-fat and highcholesterol diet (D11012302) (Research Diets, New Brunswick, USA) with 0.2\% cholesterol and fat derived from palm oil, which provided adequate levels of vitamins (Vitamin Mix V10001, Research Diets, New Brunswick, USA). The mice were divided in four experimental groups: WT mice treated with placebo $(n=12)$, WT mice treated with monoHER ( $\mathrm{n}=12), L d l r /$ mice treated with placebo $(\mathrm{n}=12)$ and $L d l r^{\circ}$ - mice treated with monoHER $(n=12)$. MonoHER was administered daily subcutaneously at a dosage of $500 \mathrm{mg} / \mathrm{kg}$ of body weight ( $25 \mu \mathrm{l}$ per g of body weight). With this dose, monoHER provided complete protection against doxorubicin-induced cardiotoxicity in mice [10]. Daily subcutaneous injection of a physiological saline solution (25 $\mu \mathrm{l} \mathrm{per}$ $\mathrm{g}$ of body weight) was used as placebo. Mice were weighed every three days and food intake was recorded for every 2 mice housed together. Diet and treatment were continued for 3 weeks, after which mice were anaesthetized with $0.05 \mathrm{mg} / \mathrm{kg}$ buprenorphine and isoflurane $0.4 \mathrm{l} / \mathrm{min}$ and sacrificed by exsanguination from the vena cava. The liver was removed and divided in different parts for further investigation. Part of the large left lobe was used for RNA isolation.

\section{Direct damage by ROS}

To determine the direct damage by ROS, oxidative damage to poly-unsaturated fatty acids in the liver was determined by measuring malondialdehyde (MDA) formation. Liver homogenate $(250 \mathrm{mg}$ liver/ml) was prepared in $145 \mathrm{mM}$ potassium phosphate buffer (pH 7.4). MDA was determined by reaction of MDA with thiobarbituric acid [11] and quantified by HPLC [12]. 


\section{Cellular redox status}

The cellular redox status was assessed by quantifying the GSH/GSSG ratio. Total glutathione (GSH) content and glutathione disulfide (GSSG) were measured according to the enzymatic recycling method [13] quantifying the conversion of 5,5-dithio-bis(2-nitrobenzoic acid) (DTNB) into 5'-thio-2-nitrobenzoic acid (TNB) spectrophotometrically at $412 \mathrm{~nm}$ and $37^{\circ} \mathrm{C}$. The GSH/GSSG-ratio was calculated from the content of GSSG and total glutathione.

\section{Activation of the NRF2 antioxidant defence system}

An adaptive response by the NRF2 antioxidant defence system was evaluated by measuring Hmox1 and Gpx3 gene expression in the liver. Liver parts that were stored in RNAlater ${ }^{\circledR}$ at $-80^{\circ} \mathrm{C}$ were used for RNA isolation using miRNeasy Mini Kit with DNase treatment (Qiagen, Venlo, The Netherlands) according to protocol. Isolated RNA was converted into complementary DNA (cDNA) using iScript cDNA synthesis kit (Bio-rad, Veenendaal, The Netherlands). Two step qRT-PCR was performed using $12.5 \mu \mathrm{l}$ SensiMix SYBR \& Fluorescein Kit (Bioline, London, UK) with $5 \mu \mathrm{l}$ cDNA, and $300 \mathrm{nM}$ of each primer (Hmox1: forward: 5'-GAGCCTGAATCGAGCAGAAC-3', reverse: 5'-CСTTCAAGGCCTCAGACAAA-3', Gpx3: forward: 5'-CATCCTGCCTTCTGTCCСТ-3', reverse: 5'-ATGGTACCACTCATACCGCC-3') in a $25 \mu \mathrm{l}$ reaction mixture. PCR was conducted as follows: denaturation at $95^{\circ} \mathrm{C}$ for 10 minutes, followed by 40 cycles of $95^{\circ} \mathrm{C}$ for 15 seconds and $60^{\circ} \mathrm{C}$ for 45 seconds. Following PCR, a melting curve (60$95^{\circ} \mathrm{C}$ ) was produced for product identification and purity check. Gapdh (glyceraldehyde-3-phosphate dehydrogenase) and Ywhaz (tyrosine 3monooxygenase/tryptophan 5-monooxygenase activation protein, zeta polypeptide) (Eurofins, Breda, The Netherlands) were used as housekeeping genes [14]. An average value of the expression of the house keeping genes Gapdh (forward: 5'TTGATGGCAACAATCTCCAC-3', reverse: 5'-CGTCCCGTAGACAAAATGGT-3') and Ywhaz (forward: 5'-CAGCAGATGGCTCGAGAATA-3', reverse: 5'-GAAGCATTGGGGATCAAGAA$\left.3^{\prime}\right)$ was used for the calculation of the $\triangle \mathrm{Ct}$-value. The validity was confirmed by the high within-animal correlation between the values of $G A P D H$ and $Y W H A Z\left(\mathrm{R}^{2}=\right.$ 0.8041). Data were analysed using the MyIQ software system (BioRad, Veenendaal, The Netherlands) and were expressed as relative gene expression (fold change) using the $2^{\Delta \Delta \mathrm{Ct}}$ method. 


\section{Statistics}

Data were analysed with GraphPad Prism 5 (Graphpad software, CA, USA). Values presented in the study are expressed as mean \pm standard deviation (SD). The relationships of the GSH/GSSG ratio with Hmox1 and Gpx3 gene expression were evaluated using linear correlation. Differences between groups were analysed using the Kruskal-Wallis test. Post-hoc testing was performed using the Mann-Whitney Utest with Bonferroni correction. A $P<0.05$ was considered to be statistically significant, $P<0.1$ and $>0.05$ as indicating a trend.

\section{Results and discussion}

There were no significant differences in food intake between the groups (table 1). Initial and also final body weight and liver weight did not differ between the groups. In the placebo treated $L d l r^{-}$- mice only a trend towards increased liver/total body weight-ratio compared to placebo treated WT mice was observed, confirming that in the relatively short period of 3 weeks only the onset of NAFLD was studied.

Table 1. Biometric data of mice.

\begin{tabular}{|c|c|c|c|c|}
\hline & $\mathbf{W T}+\mathbf{P}$ & $\mathbf{W T}+\mathbf{M H}$ & $L_{d} d r^{-/-}+\mathrm{P}$ & LdIr $^{-1-}+\mathrm{MH}$ \\
\hline Initial body weight (g) & $20.8 \pm 1.3$ & $20.9 \pm 1.1$ & $20.9 \pm 1.1$ & $21.0 \pm 1.0$ \\
\hline Final body weight (g) & $21.3 \pm 1.5$ & $21.5 \pm 1.2$ & $21.3 \pm 1.5$ & $20.8 \pm 1.5$ \\
\hline Body weight change (g) & $0.4 \pm 0.7$ & $0.6 \pm 1.1$ & $0.4 \pm 1.2$ & $-0.1 \pm 0.8$ \\
\hline Liver weight (g) & $1.00 \pm 0.14$ & $1.01 \pm 0.10$ & $1.05 \pm 0.12$ & $1.04 \pm 0.11$ \\
\hline $\begin{array}{l}\text { Liver/total body weight- } \\
\text { ratio (\%) }\end{array}$ & $4.69 \pm 0.40$ & $4.69 \pm 0.32$ & $4.95 \pm 0.35$ & $4.92 \pm 0.34$ \\
\hline $\begin{array}{l}\text { Average food intake } \\
\text { (g/day/cage) }\end{array}$ & $4.0(0.3)$ & $4.1(0.3)$ & $4.3(0.3)$ & $4.4(0.3)$ \\
\hline
\end{tabular}

Initial and final body weight, body weight change, liver weight, liver/total body weight-ratio, average food intake of WT and $L d l r /$ mice treated with placebo (P) or monoHER (MH). Mean \pm SD is shown.

A trend was seen towards a higher average level of lipid peroxidation (MDA) in $\mathrm{Ldll} /$ mice compared to WT mice (figure 1A), although this difference failed to reach significance. No pronounced effect of monoHER against ROS damage was seen. This could be due to the relatively large variation in the results. An explanation for the relatively large variation is that the onset of the disease is examined. At the onset of the disease, the effects are relatively small compared to the biological variation. Moreover, the time course of the gradual progression into a clear manifestation of the 
disease will vary between the mice studied. The effect was only examined at a single point in time, and this also contributes to a relatively large inter-animal variation. The relatively large variation might explain that only a mild effect was found on a group level.
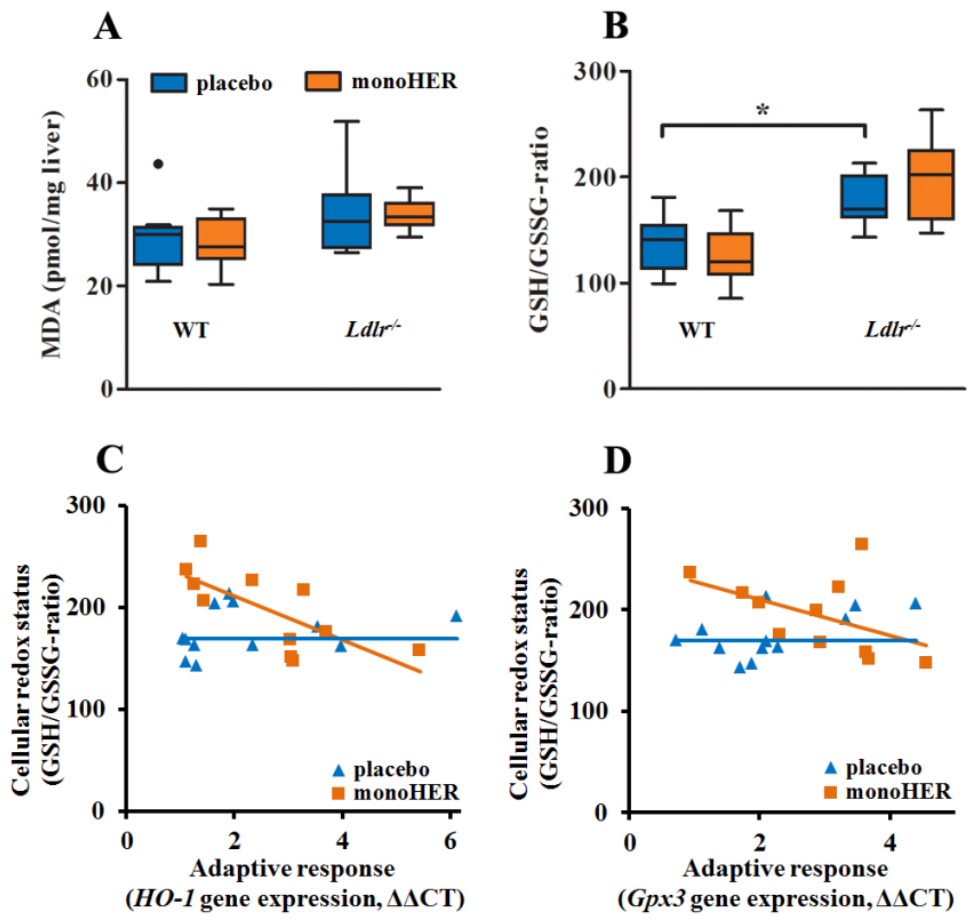

Figure 1. Direct damage by ROS (determined as MDA formation) and cellular redox status (determined as GSH/GSSG ratio). In panel A and B the Tukey Box-plot is shown of MDA and GSH/GSSG ratio, measured in liver tissue of WT and $L d l r^{\prime-}$ mice treated with placebo or MonoHER. ${ }^{*} P<0.01$. The outlier is included in the statistical evaluation. Panel $\mathrm{C}$ and $\mathrm{D}$ show the within-animal linear correlation between the cellular redox status and the NRF2-dependent adaptive response during the onset of NAFLD in the liver of $L d l /$ mice. In the group treated with MonoHER, the adaptive response, expressed as Hmox1 (C) and Gpx3 (D) gene expression, was relatively high at a low cellular redox status, indicated by a low GSH/GSSG ratio $(P=$ 0.012 and $P=0.046$ respectively). This relationship is lacking in the placebo group.

The general protective effect of monoHER against oxidative stress can be explained by its ability to scavenge ROS [7]. However, scavenging might block the activation of the innate NRF2 pathway by ROS. In this case, the paradoxical consequence is that no protective adaptation is elicited because antioxidant administration prevents the endogenous defensive shield to be raised. This has fuelled the vexed dispute on the use of antioxidants in the prevention and treatment of diseases [15]. However, in the 
present study it was found that monoHER did not block the adaptive response; in contrast, a trend towards further increased levels of Hmox1 and Gpx3 gene expression was found with monoHER (table 2).

Table 2. Activation of the NRF2 antioxidant defence system.

\begin{tabular}{|c|c|c|c|c|}
\hline Gene & $\mathbf{W T}+\mathbf{P}$ & $\mathbf{W T}+\mathbf{M H}$ & $L d l r^{-/-}+\mathrm{P}$ & $L_{d} I r^{-1-}+\mathrm{MH}$ \\
\hline Hmox1 & $1.00 \pm 0.40$ & $0.98 \pm 0.29$ & $2.27 \pm 1.48^{*}$ & $2.66 \pm 1.25 * *$ \\
\hline Gpx3 & $1.00 \pm 0.57$ & $1.43 \pm 0.51$ & $2.21 \pm 1.01 *$ & $3.02 \pm 1.09 * *$ \\
\hline
\end{tabular}

Hmox1 and Gpx3 gene expression was measured to evaluate the activation of the NRF2 antioxidant defence system in liver tissue of WT and Ldlr/ mice treated with placebo (P) or MonoHER (MH). Data represent fold change compared to WT + p group. Mean \pm SD is shown. ${ }^{*} P<0.05$ compared to WT + p group, ${ }^{* *} P<0.05$ compared to WT + MonoHER group.

The higher NRF2 induced gene expression in Ldlr/- mice (table 2) was corroborated by the adaptive response on the cellular redox status (figure 1B). In $\mathrm{Ldlr} /$ mice monoHER also tended to further increase the GSH/GSSG-ratio in Ldlr $/$ mice (figure 1B).

That monoHER does not inhibit the adaptive response can be explained by the formation of a thiol reactive oxidation product of monoHER when it scavenges radicals. This oxidation product of monoHER selectively adducts a cysteine residue in KEAP1 and this adduction of KEAP1 induces the adaptive response [8]. In fact, the rather unspecific reactivity of ROS that does not only destroy KEAP1 but also vital cellular components such as other proteins and DNA, is exchanged for the much more selective reactivity of the oxidized product of monoHER towards KEAP1 [8].

MonoHER administration did not induce an adaptive response in the WT animals. In this respect, it should be noted that monoHER itself lacks thiol reactivity. MonoHER first needs to be converted into a thiol-reactive oxidation product to induce an adaptive response [8]. This conversion happens when monoHER scavenges radicals. In the control animals the generation of the thiol-reactive oxidation product will be relatively low. Therefore, no adaptive response as a result of monoHER administration is expected in the control animals.

The formation of the thiol-reactive oxidation product, exclusively during oxidative stress can be seen as a form of targeting. It results in an adaptive enforcement of the antioxidant system when this is needed. Interestingly, in the monoHER treated $\mathrm{Ldlr}^{\circ}$ - 
mice also evidence for targeting was seen. We expected that monoHER would activate NRF2 which subsequently would results in a higher cellular redox status. This was indeed found, but to our surprise in the monoHER group an improved redox status was associated with a relatively low NRF2 activation. Apparently, monoHER administration results in a relatively potent adaptation. In the monoHER treated $L d l r$ 1- mice NRF2 activation was relatively high at a low GSH/GSSG ratio. This indicates that a relatively low redox status, i.e. a low GSH/GSSG ratio, tends to increase NRF2 activation in the monoHER treated $L d l r^{/-}$group. Moreover, in the liver of animals that have a relatively high GSH/GSSG ratio, no evidence for further stimulation of NRF2 by monoHER was seen, indicating that in the animals that have adapted, no further adaptation is needed (figure $1 \mathrm{C}$ and $1 \mathrm{D}$ ). In retrospect, this relationship is more logical than the anticipated high redox status when the adaptive response is high, i.e. that in the adapted animals more adaptation is needed, which of course is not necessary. No correlation was found in the corresponding placebo group and in both control groups with WT mice probably because there was no substantiated adaptation. The higher level of adaptation and the reported correlation with NRF2 activation in monoHER treated $\mathrm{Ldlr}^{-}$- mice was absent in the other group. This indicates that monoHER has promoted the adaptive response.

In the present in vivo study, only a mild protective effect of monoHER against oxidative stress was seen. As mentioned above, this may be related to the relatively low level of oxidative stress and relatively large variation in the lipid peroxidation marker (figure $1 \mathrm{~A}$ ) because the onset of pathology was examined. An additional reason for this mild protection is that monoHER is administered once a day, whereas the half-life of monoHER is only half an hour [16]. Most of the time, monoHER will be practically absent [17], and for full protection by radical scavenging the compound has to be present continuously at a relatively high concentration. Scavenging of radicals at moments when concentrations of monoHER are relatively high, can explain the subtle effect of monoHER administration on oxidative stress. In addition, as mentioned above, the scavenging of radicals and the concurrent formation of the thiol-reactive oxidation product can selectively activate the innate NRF2 machinery. As demonstrated previously, a single and acute exposure to a thiol-alkylating agent can induce an adaptive response [18-20]. This would mean that in order for monoHER to induce an adaptive response, only part of the radicals needs to be scavenged since this already leads to the formation of the thiol-alkylating oxidation product of monoHER. 
Flavonoids have therapeutic potential in NAFLD which relates to their protection against oxidative stress. Our study supports a modified mechanism of action of the antioxidant flavonoid monoHER, which might also apply for other free radical scavenging antioxidants, as illustrated in Figure 2. The results show that the direct scavenging of radicals is only one aspect of protection by monoHER. The present study shows that activation of the antioxidant defence system (i.e. the activation of NRF2) by the oxidized flavonoid also plays a crucial role in the protective effect against oxidative stress (i.e. the improved GSH/GSSG ratio). This molecular mechanism does not only result in a very potent antioxidant effect, it also ensures that monoHER will act selectively in a condition of oxidative stress. In this way monoHER empowers the buffering capacity of the body to maintain homeostasis and to prevent that relatively mild but nevertheless insidious and persistent perturbations gradually progresses into a severe pathology, such as NAFLD.

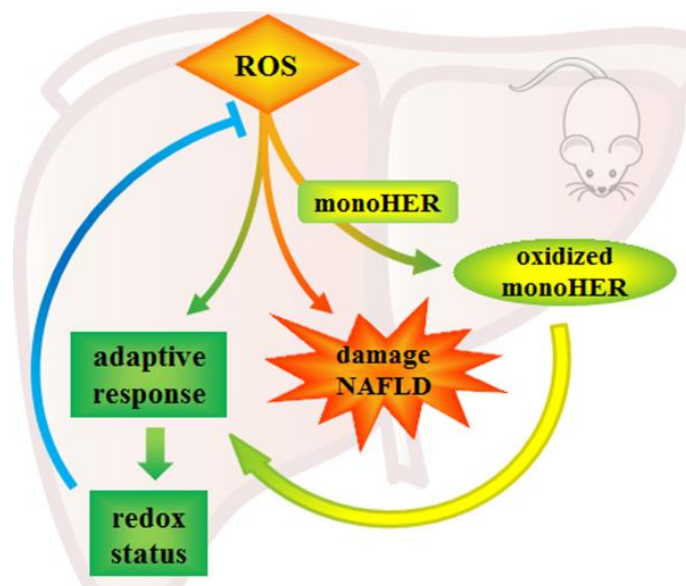

Figure 2. Overview of the protective effect of MonoHER in the onset of NAFLD in mice. The high and therefore non selective reactivity of ROS results in damage to virtually any cellular compound. This oxidative damage is a hallmark in the etiology of NAFLD. ROS also activate Nrf2 which leads to adaptation. By scavenging the ROS, oxidation products of MonoHER are formed that promote the adaptation of the cell to oxidative stress. This helps to maintain homeostasis and prevents that relatively mild perturbations gradually progress into a severe pathology such as NAFLD. 


\section{Acknowledgements}

The authors would like to thank Leonie Jonkers (Department of Toxicology, Faculty of Health, Medicine and Life Sciences, Maastricht University, P.O. Box 616, 6200 MD Maastricht, The Netherlands) for the help with the treatment of the mice. We are also thankful to Kelly Waagmeester (Department of Toxicology, Faculty of Health, Medicine and Life Sciences, Maastricht University, P.O. Box 616, 6200 MD Maastricht, The Netherlands) for the help with the RNA isolation and measuring of the gene expression. 


\section{References}

1. Berlanga, A., et al., Molecular pathways in non-alcoholic fatty liver disease. Clinical and experimental gastroenterology, 2014. 7: p. 221-39.

2. Koek, G.H., P.R. Liedorp, and A. Bast, The role of oxidative stress in non-alcoholic steatohepatitis. Clinica chimica acta; international journal of clinical chemistry, 2011. 412(15-16): p. 1297-305.

3. Rolo, A.P., J.S. Teodoro, and C.M. Palmeira, Role of oxidative stress in the pathogenesis of nonalcoholic steatohepatitis. Free radical biology \& medicine, 2012. 52(1): p. 59-69.

4. Itoh, K., K.I. Tong, and M. Yamamoto, Molecular mechanism activating Nrf2-Keap1 pathway in regulation of adaptive response to electrophiles. Free radical biology \& medicine, 2004. 36(10): p. 1208-13.

5. What is health? The ability to adapt. Lancet, 2009. 373(9666): p. 781.

6. van de Wier, B., et al., The potential of flavonoids in the treatment of non-alcoholic fatty liver disease. Critical Reviews in Food Science and Nutrition.: p. in press.

7. Lemmens, K.J., et al., The flavonoid 7-mono-O-(beta-hydroxyethyl)-rutoside is able to protect endothelial cells by a direct antioxidant effect. Toxicology in vitro : an international journal published in association with BIBRA, 2014. 28(4): p. 538-43.

8. Lemmens, K.J., et al., The antioxidant flavonoid monoHER provides efficient protection and induces the innate Nrf2 mediated adaptation in endothelial cells subjected to oxidative stress. PharmaNutrition, 2014.

9. Bieghs, V., et al., LDL receptor knock-out mice are a physiological model particularly vulnerable to study the onset of inflammation in non-alcoholic fatty liver disease. PLoS One, 2012. 7(1): p. e30668.

10. van Acker, S.A., et al., Monohydroxyethylrutoside as protector against chronic doxorubicin-induced cardiotoxicity. British journal of pharmacology, 1995. 115(7): p. 1260-4.

11. Haenen, G.R.M.M. and A. Bast, Protection against lipid peroxidation by a microsomal glutathione-dependent labile factor. FEBS letters, 1983. 159(1-2): p. 24-28.

12. Lepage, G., et al., Preparative steps necessary for the accurate measurement of malondialdehyde by high-performance liquid chromatography. Analytical biochemistry, 1991. 197(2): p. 277-83.

13. Julicher, R.H., et al., The effect of chronic adriamycin treatment on heart kidney and liver tissue of male and female rat. Archives of toxicology, 1988. 61(4): p. 275-81.

14. Bruce, K.D., et al., The housekeeping gene YWHAZ remains stable in a model of developmentally primed non-alcoholic fatty liver disease. Liver International, 2012. 32(8): p. 1315-1321.

15. Bast, A. and G.R. Haenen, Ten misconceptions about antioxidants. Trends in pharmacological sciences, 2013. 34(8): p. 430-6.

16. Abou El Hassan, M.A., et al., Bioavailability and pharmacokinetics of the cardioprotecting flavonoid 7-monohydroxyethylrutoside in mice. Cancer Chemother Pharmacol, 2003. 52(5): p. 371-6.

17. Jacobs, H., et al., Identification of the metabolites of the antioxidant flavonoid 7-mono-O(beta-hydroxyethyl)-rutoside in mice. Drug Metab Dispos, 2011. 39(5): p. 750-6.

18. Sthijns, M.M., et al., Adaptation to acrolein through upregulating the protection by glutathione in human bronchial epithelial cells: the materialization of the hormesis 
concept. Biochemical and biophysical research communications, 2014. 446(4): p. 1029-34.

19. Wakabayashi, N., et al., Protection against electrophile and oxidant stress by induction of the phase 2 response: fate of cysteines of the Keap1 sensor modified by inducers.

Proceedings of the National Academy of Sciences of the United States of America, 2004. 101(7): p. 2040-5.

20. Levonen, A.L., et al., Redox regulation of antioxidants, autophagy, and the response to stress: implications for electrophile therapeutics. Free radical biology \& medicine, 2014. 71: p. 196-207. 



\title{
Chapter 6
}

\section{Chemical characteristics for optimizing CYP2E1 inhibition}

\author{
Bregje van de Wier ${ }^{*}$, Jiska M. Balk, Aalt Bast, \\ Ger H. Koek, Guido R.M.M. Haenen
}

Chemico-Biological Interactions. 2015; 242:139-44.

* equal contribution 


\section{Abstract}

Cytochrome P450 2E1 (CYP2E1) expression and activity in the liver is associated with the degree of liver damage in patients with alcoholic steatohepatitis (ASH) as well as non-alcoholic steatohepatitis (NASH). CYP2E1 is known to generate reactive oxygen species, which leads to oxidative stress, one of the hallmarks of both diseases. Apart from ROS, toxic metabolites can be formed by CYP2E1 metabolism, further potentiating liver injury. Therefore, CYP2E1 is implicated in the pathogenesis of ASH and NASH.

The aim of this study was to determine the chemical characteristics of compounds that are important to inhibit CYP2E1. To this end, structurally related analogues that differed in their lipophilic, steric and electronic properties were tested. In addition, homologues series of aliphatic primary alcohols, secondary alcohols, aldehydes, ketones and carboxylic acids were tested. It was found that inhibition of the CYP2E1 activity is primarily governed by lipophilicity. The optimal $\log \mathrm{D}_{7.4}$ (octanol/water distribution coefficient at pH 7.4) value for inhibition of CYP2E1 was approximately 2.4. In the carboxylic acids series the interaction of the carboxylate group with polar residues lining the CYP2E1 active site also has to be considered.

This study sketches the basic prerequisites in the search for inhibitors of CYP2E1, which would strengthen our therapeutic armamentarium against CYP2E1 associated diseases, such as ASH and NASH. 


\section{Introduction}

Alcoholic steatohepatitis (ASH) is a common liver disorder characterised by steatosis and inflammation seen in patients that abuse alcohol. Its exact prevalence is unknown, but it is estimated to be present in $10-35 \%$ of hospitalized alcoholics [1]. Patients with ASH have a poor prognosis; up to $50 \%$ of patients develop cirrhosis. Abstinence from alcohol does not guarantee full recovery; only in $27 \%$ of patients histological normalisation is found, whereas in $18 \%$ cirrhosis develops and in the remaining patients ASH persists [2].

Non-alcoholic steatohepatitis (NASH) is a liver disorder with histological similarity to ASH, seen in patients with no history of alcohol abuse (maximal 2 units per day for men and 1 unit per day for women). It is often associated with the metabolic syndrome and obesity [3-5]. NASH is diagnosed in $2-3 \%$ of the general adult population in developed countries [6]. In NASH, 15 to $25 \%$ of patients will eventually develop cirrhosis [7] due to persistent inflammation in which oxidative stress plays an important role [8].

Both ASH and NASH have in common that the expression and activity of the enzyme cytochrome P450 2E1 (CYP2E1) are up-regulated [9-12]. CYP2E1 is the main cytochrome P450 of the microsomal ethanol oxidizing system (MEOS) and has a role in the metabolism of xenobiotics. It accepts a broad variety of substrates, including ethanol, ketones and fatty acids [12]. These substrates accumulate in ASH and NASH, which could explain the induction of the enzyme [12]. Several studies have observed an association between the increase in CYP2E1 expression and activity in the liver and the degree of liver damage in patients with ASH and NASH [13-15].

CYP2E1 is known to generate reactive oxygen species (ROS) [12, 16, 17]. Superoxide $\left(\mathrm{O}_{2}{ }^{-}\right)$and hydrogen peroxide $\left(\mathrm{H}_{2} \mathrm{O}_{2}\right)$ are formed during the catalytic cycle of CYP2E1 $[11,18,19]$. In the pathogenesis of both ASH and NASH, there is a central role for the production of ROS and the induction of oxidative stress and subsequent liver damage. It was previously shown that CYP2E1 activity correlates with ethanol induced liver damage and lipid peroxidation [20]. Therefore CYP2E1 activity is implicated in the pathogenesis of these diseases. Besides ROS, CYP2E1 can also form other toxic metabolites, further potentiating liver injury [21]. 
Because previous studies have suggested that the production of ROS and the formation of toxic metabolites by cytochrome P4502E1 are related to its monooxygenase activity $[11,18,19,22]$, inhibition of the CYP2E1 monooxygenase activity might reduce the oxidative stress caused by CYP2E1.

The aim of this study was to determine the impact of lipophilic, steric and electronic properties of compounds on the inhibition of CYP2E1 activity. This will give the basis prerequisites for inhibitors of CYP2E1, which might be used in the treatment of both ASH and NASH, diseases still lacking effective pharmacotherapeutic treatment.

\section{Materials and methods}

\section{Chemicals}

4-Nitrophenol, 4-nitrocatechol, phenol, 4-chlorophenol, 3,4-dichlorophenol, 2,4dichlorophenol, 4-(trifluoromethyl)phenol, glucose-6-phosphate dehydrogenase, nicotinamide adenine dinucleotide phosphate (NADP+), D-glucose-6-phosphate, trichloro-acetic acid (TCA), primary alcohols, secondary alcohols, 2-ketones, aldehydes and carboxylic acids (all up to a chain length of eight carbon atoms) were obtained from Sigma-Aldrich (St. Louis, USA). Magnesiumchloride, di-potassium hydrogen phosphate and potassium dihydrogen phosphate were purchased from Merck (Darmstadt, Germany). Anti-CYP2E1 antibodies for Western Blot were obtained from Millipore (Temecula, USA), horseradish peroxidase-conjugated secondary antibodies were purchased from Cell Signaling Technology (Danvers, USA) and non-fat dry milk (NFDM) was obtained from Biorad (Veenendaal, the Netherlands). Anti-CYP2E1 antibodies were kindly provided by prof. dr. M. Ingelman-Sundberg (Karolinska institute).

\section{Preparation of rat liver microsomes}

Lewis rats were treated to induce CYP2E1 in the liver. Therefore, the rats received drinking water that contained $1 \%(\mathrm{v} / \mathrm{v}$ ) acetone ad libitum for seven days. Twenty four hours before sacrifice, the food was removed to further induce CYP2E1 in the liver [22]. The rats were anaesthetized with $\mathrm{CO}_{2} / \mathrm{O}_{2}$ (ratio 2/3) and decapitated. The study was approved by the institutional ethical review board on experiments with laboratory animals. 
The livers were isolated and rinsed in ice-cold $50 \mathrm{mM}$ potassium phosphate buffer $\mathrm{pH}$ 7.4) containing $22.8 \mathrm{mM}$ potassium chloride and $3.4 \mathrm{mM}$ EDTA. The livers were homogenized and centrifuged at $10000 \mathrm{x}$ g for 20 minutes at $4{ }^{\circ} \mathrm{C}$. The supernatant was centrifuged at $65000 \mathrm{x}$ g for 60 minutes at $4 \stackrel{\circ}{ } \mathrm{C}$. The supernatant was removed and the remaining pellet was resuspended in buffer. This solution was centrifuged at $100000 \mathrm{x}$ g for 50 minutes at $4 \stackrel{\circ}{\circ}$. The supernatant was removed and the pellet was resuspended in buffer to obtain $2 \mathrm{~g}$ liver per $\mathrm{ml}$ buffer. This corresponded to $15 \mathrm{mg}$ protein $/ \mathrm{ml}$ buffer. The microsomes that were obtained in this way were stored at -80 ${ }^{\circ} \mathrm{C}$ until use.

\section{Western Blot}

Western Blot was performed to confirm the induction of CYP2E1. Equal amounts of protein from CYP2E1 induced samples and from control samples were separated by SDS-polyacrylamide gel electrophoresis (4-15\% TGX gel, BioRad) and transferred to Hybond ECL nitrocellulose membranes (GE Healthcare) using the Criterion blotting system (Biorad). Protein concentrations were determined with the use of bicinchoninic acid according to Smith et al [23]. Membranes were blocked with 5\% NFDM. NFDM was diluted in washing buffer $(0.05 \%$ Tween-20 in Tris Buffered Saline). After blocking, membranes were incubated overnight with anti-CYP2E1 antibody diluted 1:1000 in 5\% NFDM in washing buffer. Horseradish peroxidaseconjugated secondary antibody diluted 1:2000 in 5\% NFDM in washing buffer was used for detection of immunoreactive proteins by chemiluminescence (Thermo Scientific).

\section{Determination of CYP2E1 activity}

4-nitrophenol was used to determine CYP2E1 activity. This substrate is converted to 4-nitrocatechol by CYP2E1. Concentrations of 4-nitrocatechol formed and 4nitrophenol remaining were measured by HPLC as described by Elbarbry et al. [24] with some modifications.

A reaction mixture of $175 \mu \mathrm{l}$ was prepared containing a certain 4-nitrophenol concentration and a NADPH generating system (consisting of $3.3 \mathrm{mM} \mathrm{MgCl} 2,3.3 \mathrm{mM}$ glucose-6-phosphate, $1.3 \mathrm{mM} \mathrm{NADP+}$ and 0.4 units/ml glucose-6-phosphate dehydrogenase) with or without an inhibitor dissolved in a $50 \mathrm{mM}$ potassiumphosphate buffer ( $\mathrm{pH}$ 7.4). 
The reaction mixture and rat liver microsomes were preincubated in separate tubes at $37 \stackrel{\circ}{\mathrm{C}}$ for 5 minutes. After 5 minutes, $25 \mu \mathrm{l}$ of the rat liver microsomes were added to the reaction mixture and the tube was incubated at $3{ }^{\circ} \stackrel{\circ}{C}$ for 4 minutes. The reaction was stopped by adding $100 \mu \mathrm{l}$ of $10 \%$ TCA to the tube and vortexing the tube. After centrifuging at $12000 \mathrm{x}$ g for 4 minutes, the supernatant was injected into the HPLC (reversed phase Altima ${ }^{\mathrm{TM}}$ C18 column, mobile phase $30 \%$ acetonitrile and $70 \%$ milliQ with $0.1 \% \mathrm{TFA}$ ) to determine the concentrations 4-nitrophenol and 4-nitrocatechol. HPLC flow rate was $1 \mathrm{ml} / \mathrm{min}$ and the run time was 5 minutes. The UV-absorbance was measured at $350 \mathrm{~nm}$. CYP2E1 activity was also investigated in control samples and in CYP2E1 induced samples in the presence of CYP2E1 antibodies to confirm the role of CYP2E1 in 4-nitrophenol hydroxylation.

\section{Inhibitor testing studies}

The Topliss decision tree [25] was used to determine which chemical characteristic is most important for inhibiting CYP2E1. This scheme consists of compounds with lipophilic, electronic and steric differences. By comparing the activity of different compounds, one can determine which characteristic of a substance is most important for its activity.

Phenol and 4-chlorophenol were the first compounds tested. Depending on the inhibitory potential of 4-chlorophenol in comparison to the inhibitory potential of phenol, other substances of the Topliss scheme were tested. The inhibitory activity of compounds tested in the Topliss scheme was expressed as per cent of normal CYP2E1 activity without using inhibitors.

In addition to the compounds of the Topliss scheme, groups of carboxylic acids, primary alcohols, secondary alcohols, ketones and aldehydes with similar carbon chain lengths were tested on their inhibitory potential. Compounds with a $\log \mathrm{D}_{7.4}$ up to 3.0 were tested. For charged compounds, the $\log \mathrm{D}_{7.4}$ was calculated using MarvinSketch 5.12.4. For uncharged compounds the LogP value of the PubChem Compound database was used. For uncharged compounds the $\log \mathrm{D}_{7.4}$ is similar to the $\log \mathrm{P}$ value. The inhibitory activity was expressed as $\mathrm{IC}_{50}$, the concentration of an inhibitor that reduces the enzyme activity by half. 


\section{Results}

\section{CYP2E1 induction}

Western Blot confirmed the induction of CYP2E1 in liver microsomes of rats that were treated to induce CYP2E1 (figure 1). Para-nitrophenol hydroxylation activity was much higher in CYP2E1 induced microsomes than in control microsomes from untreated rat (figure 2). Also, the 4-nitrophenol hydroxylation activity was almost completely inhibited by CYP2E1 antibodies (figure 2), confirming the role of mainly CYP2E1 in 4-nitrophenol hydroxylation.

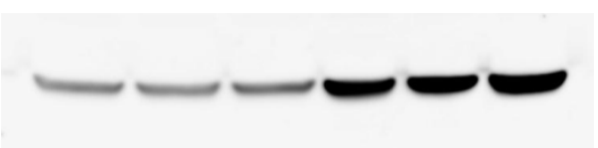

Figure 1. Western blot of CYP2E1. Western blot of rat CYP2E1 in microsomes of rats that were treated to induce CYP2E1 (samples 1-3) compared to control (samples 4-6).

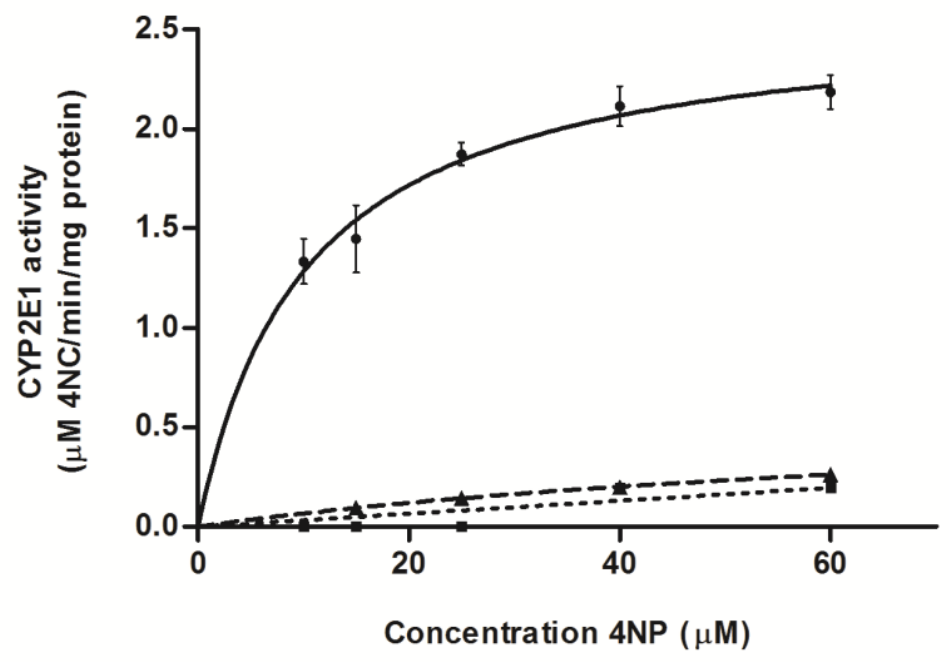

Figure 2. CYP2E1 activity. Michaelis-Menten curve of CYP2E1 activity measured by the conversion of 4nitrophenol (4NP) into 4-nitrocatechol (4NC) in rat microsomes treated to induce CYP2E1 (uninterrupted line), control microsomes (interrupted line) and CYP2E1 induced microsomes in the presence of CYP2E1 antibodies (dotted line). The results are expressed as mean $\pm \mathrm{SEM}, \mathrm{n}=2$. 


\section{CYP2E1 inhibitor testing according to the Topliss scheme}

4-Chlorophenol was more effective in inhibiting 4-nitrophenol hydroxylation than phenol (60\% less formation of 4-nitrocatechol compared to control by 4chlorophenol, versus $18 \%$ inhibition by phenol) (figure 3). According to the Topliss scheme used, 3,4-dichlorophenol, 4-trifluoromethylphenol and 2,4-dichlorophenol were tested next (figure 3A). All these compounds were about equally effective in inhibiting 4-nitrophenol hydroxylation (42\%, 46\% and $43 \%$ respectively), but were less potent CYP2E1 inhibitors than 4-chlorophenol (figure 3A and B). 4-Nitrophenol was the reference compound that was used in the competition experiment for CYP2E1 inhibition, and therefore the activity of 4-nitrophenol in the scheme is, by definition, $50 \%$. 


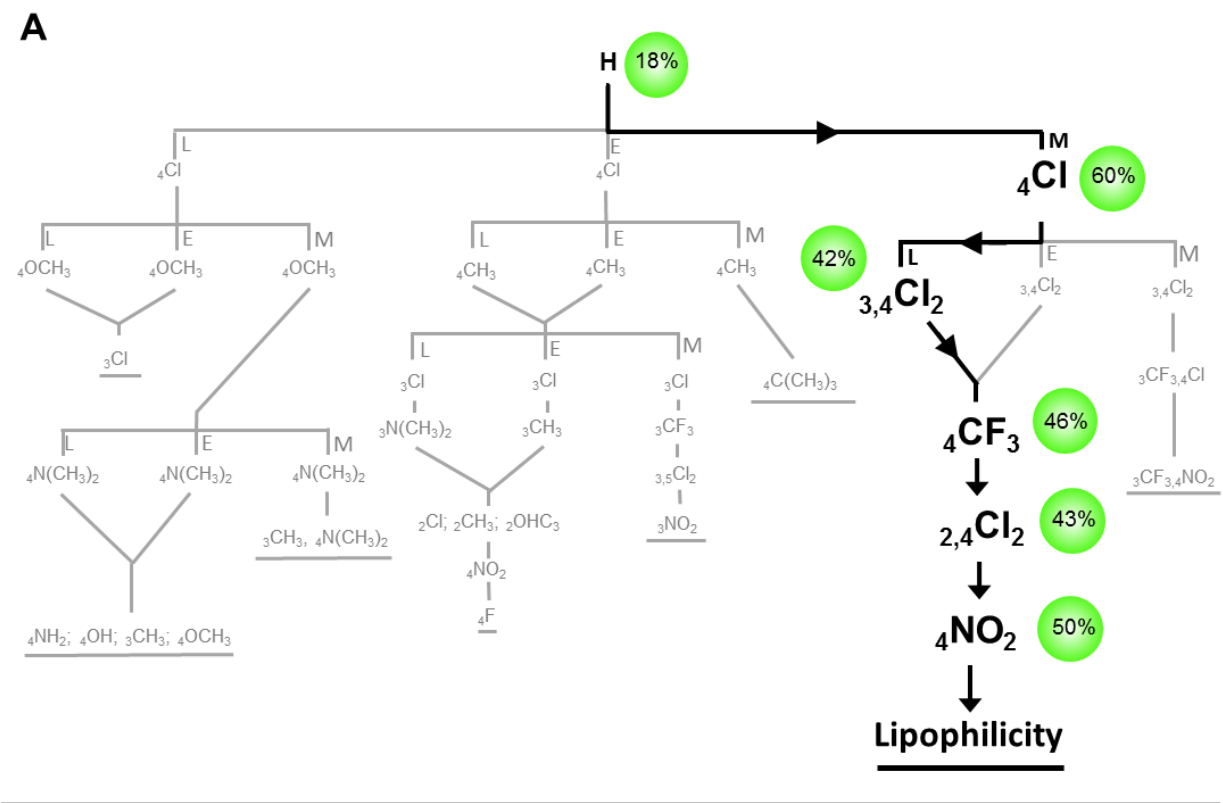

B

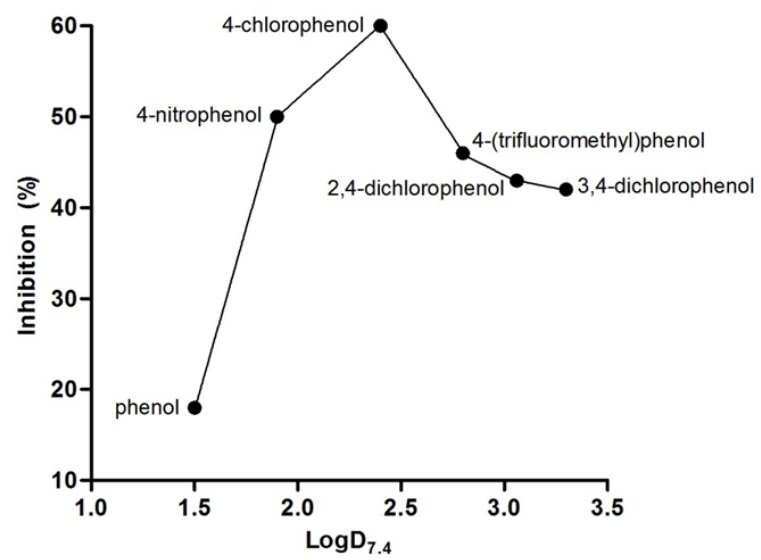

Figure 3. A) The Topliss scheme for CYP2E1 inhibition. The Topliss scheme is a decision tree to determine whether electronic, steric or lipophilic properties are the most important characteristics of compounds for the activity that is studied. At each level of the decision tree, the activity of a compound is compared with a structurally related compound. Their relative activity is given in the scheme: $\mathrm{L}=$ less active, $\mathrm{E}=$ equally active, $\mathrm{M}=$ more active. The percentage of CYP2E1 inhibition by the compounds is given is the green circles. In the end, the decision tree gives the characteristic (electronic, steric or lipophilic) that is most important for the activity studied. The lead compound was phenol $(\mathrm{H})$. At the first level 4-chlorophenol $\left({ }_{4} \mathrm{Cl} ; 60 \%\right.$ inhibition under the conditions used) was more active (M) than phenol (18\% inhibition), indicating that the right branch of the tree had to be followed. At the second level, 3,4-dichlorophenol ${ }_{3,4} \mathrm{Cl}_{2} ; 46 \%$ inhibition $)$ was less potent (L) than 4-chlorophenol, indicating that the left branch had to be taken. According to the scheme, 4-(trifluoromethyl)phenol $\left({ }_{4} \mathrm{CF}_{3}\right), 2$,4-dichlorophenol $\left(2,4 \mathrm{Cl}_{2}\right)$ and 4-nitrophenol $\left({ }_{4} \mathrm{NO}_{2}\right)$ were also 
tested. The Topliss scheme shows that lipophilicity is the most important characteristic for CYP2E1 inhibition. B) Percentage of inhibition of the tested compounds depicted against the LogD7.4 value.

\section{Inhibitory potential of carboxylic acids, primary alcohols, secondary alcohols, ketones and aldehydes}

Primary alcohols, secondary alcohols, ketones and aldehydes were all good inhibitors of 4-nitrophenol hydroxylation (figure 4). Carboxylic acids showed the least inhibitory potential, while primary alcohols were the most potent inhibitors. For primary and secondary alcohols, ketones and aldehydes, the length of the alkyl chain paralleled with the inhibitory potential (figure 4).

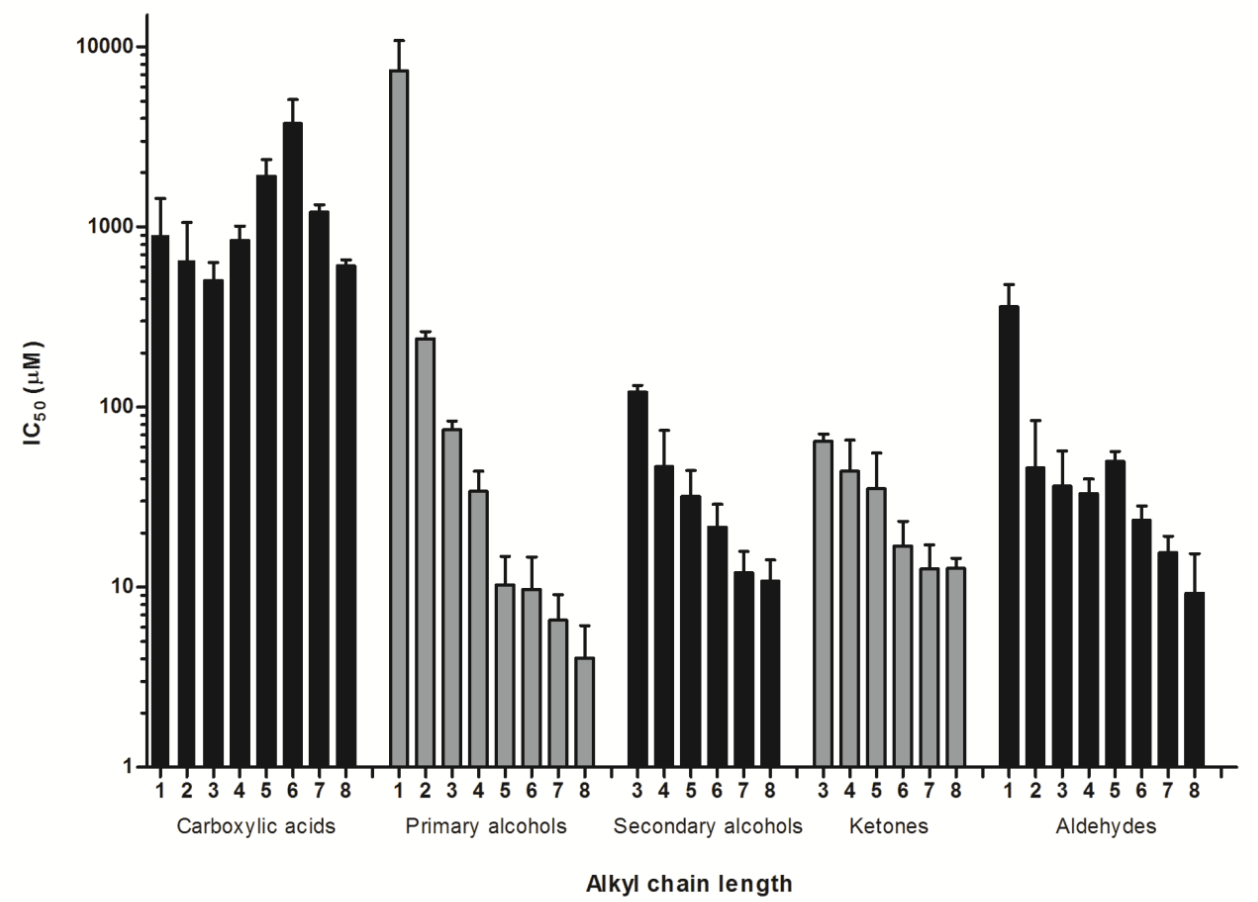

Figure 4. CYP2E1 inhibitors. The $\mathrm{IC}_{50}$ value, the concentration of inhibitor that reduces CYP2E1 activity by $50 \%$, of groups of carboxylic acids, primary alochols, secondary alcohols, ketones and aldehydes, is depicted. The results are expressed as mean $\pm \mathrm{SD}, \mathrm{n}=2$. 


\section{Discussion}

In its catalytic function, CYP2E1 can inflict damage to the liver by the formation of reactive metabolites, such as the 1-hydroxyethyl radical from ethanol, or by the production of ROS [26]. The formation of these reactive metabolites and ROS is implicated in the development of both alcoholic and non-alcoholic fatty liver disease, conditions in which CYP2E1 is found to be induced [26]. Inhibition of CYP2E1 activity is expected to inhibit the formation of these noxious compounds.

This study was aimed to elucidate the chemical characteristics needed for binding to the active site of CYP2E1. This knowledge forms the fundamental basis to select lead compounds that prevent the formation of reactive metabolites and ROS by CYP2E1.

Our study demonstrates that lipophilicity is most important for binding to the CYP2E1 active site and inhibiting CYP2E1 monooxygenase activity. In our study, the Topliss approach was applied [25]. In this approach, relatively simple compounds can be used to rank the impact of lipophilic, electronic and steric effects. The monooxygenase activity of CYP2E1 determined by 4-nitrophenol hydroxylation was used as a measure of binding to the CYP2E1 active site. As inhibition of the 4-nitrophenol hydroxylation increases, the affinity of the compound for the active site of CYP2E1 is higher.

Of the tested compounds, 4-chlorophenol appeared to be the most potent inhibitor of the 4-nitrophenol monooxygenase activity. This indicates the involvement of primarily lipophilic or electronic effects. The lower potency of 3,4-dichlorophenol compared to 4-chlorophenol points to an optimum in lipophilicity. The practically equal activity of 2,4-dichlorophenol and 4-(trifluoromethyl)phenol dismisses an unfavorable steric effect of meta substitution.

Apparently, there is an optimum in lipophilicity for binding to the CYP2E1 active site. This is corroborated by the superior potency of 3,4-dichlorophenol and 2,4dichlorophenol compared to 4-(trifluoromethyl)phenol that has a somewhat lower lipophilicity (fig. 3B). The optimum $\log \mathrm{D}_{7.4}$ value (equal to the $\log \mathrm{P}$ value for uncharged compounds) for CYP2E1 inhibition is, based on the superior activity of 4chlorophenol, approximately 2.4. This is in good accordance with previous quantitative structure-activity relationship studies of C1-C10 primary alcohols and C6-C16 carboxylic acids, reporting an optimum logP value for CYP2E1 inhibition of approximately 2.5 [27]. 
We extended our research by examining a homologues series of primary alcohols, secondary alcohols, ketones and aldehydes. All tested compounds were relatively good inhibitors of the monooxygenase activity of CYP2E1. Interestingly, the inhibitory potency increased with the length of the alkyl chain, which correlates with increasing lipophilicity (figure 5). A similar relationship was found by Martikainen et al. for primary alcohols [28] and by Lewis et al. for primary and secondary alcohols [29].

Carboxylic acids displayed relatively weak inhibitory activity, while fatty acids are endogenous substrates of CYP2E1. However, for the fatty acids tested (C1-C8), $\omega$ hydroxylation by CYP2E1 is not required in vivo, and C1-C8 fatty acids are primarily metabolized by beta-oxidation in mitochondria. Mainly medium to long chain fatty acids (C9-C20) are oxidized by CYP2E1 [30].

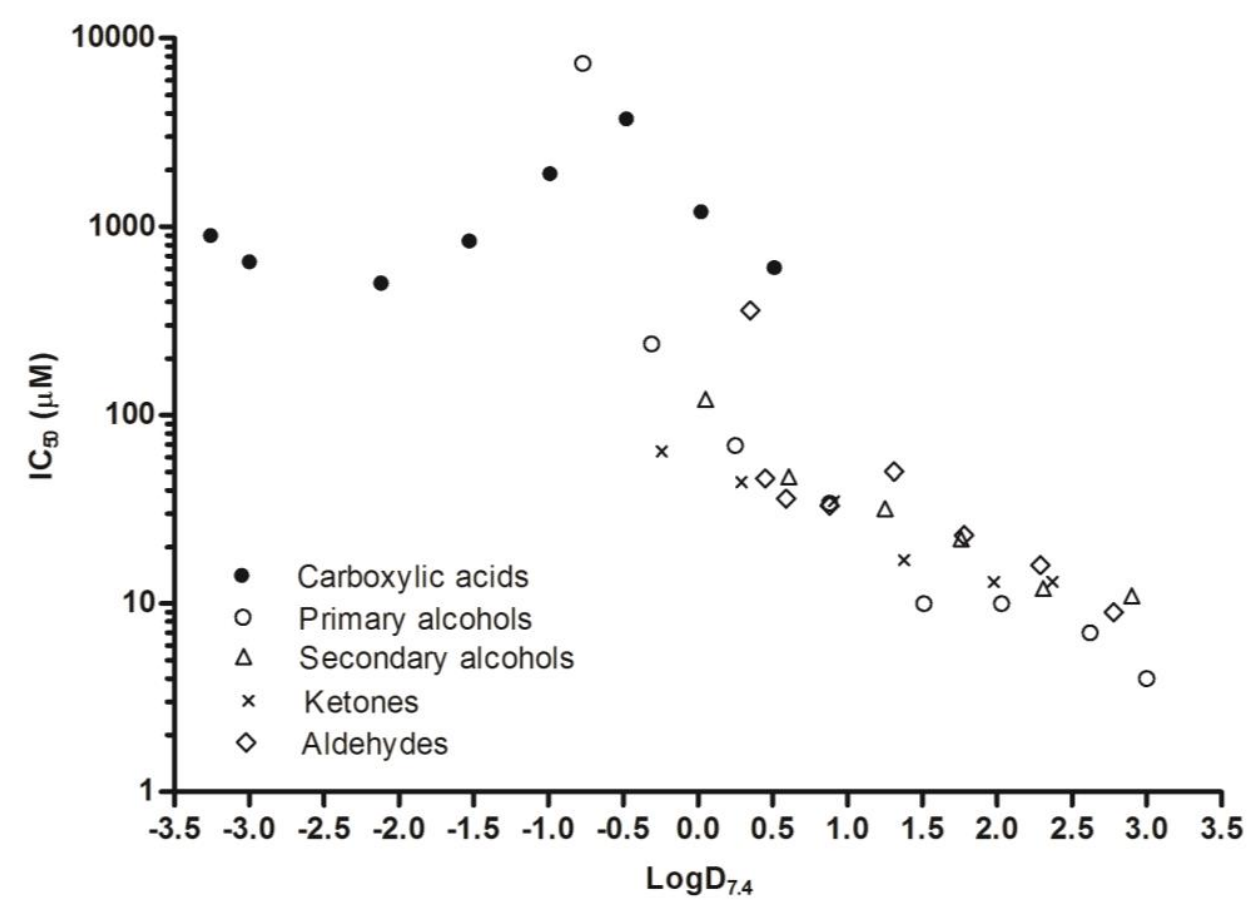

Figure 5. Lipophilicity of CYP2E1 inhibitors. Relation between the lipophilicity of the tested compounds $\left(\log \mathrm{D}_{7,4}\right)$ and the IC50 of CYP2E1 inhibition. The groups of compounds tested are carboxylic acids $(\bullet)$, primary alcohols $(0)$, secondary alcohols $(\Delta)$, ketones $(x)$ and aldehydes $(\diamond)$. 
Interestingly, for carboxylic acids, small chain molecules (C1-C4) were superior to longer chain molecules ( $\mathrm{C} 5-\mathrm{C} 6)$, but at a chain length above seven carbon atoms, the inhibitory potential increased. This is probably caused by the negative charge of the carboxylate moiety, while the other moieties are neutral. Previous studies reported that the negatively charged carboxyl group is most likely positioned outside the largely nonpolar active site of CYP2E1 [17]. The only polar residue in the vicinity of the active site is $\mathrm{Thr}^{303}$ (figure 6) [17].The carboxyl groups of small fatty acids (C1-C4) might bind to $\mathrm{Thr}^{303}$. The relatively potent inhibition of the C1-C4 fatty acids can be explained by binding of the carboxylate group to the $\mathrm{Thr}^{303}$ residue, located near the active site. The longer fatty acids (C5-C8) do not interact with the Thr ${ }^{303}$ residue, but with the Asn ${ }^{206}$ residue that is positioned further away from the active site (figure 6). The latter interaction has been found for $\mathrm{C} 8$ and $\mathrm{C} 10$ fatty acid analogs containing a charged imidazole moiety [30]. When the carboxylate group is bound to Asn ${ }^{206}$, the C5 and $\mathrm{C} 6$ fatty acids are too short to bridge the distance between Asn ${ }^{206}$ and the active site of CYP2E1, as modeled in figure 7. The C7 and C8 fatty acids are able to bridge this distance and block the active site for 4-nitrophenol (figure 7). This explains the inhibition pattern of the carboxylic acids found in the present study.

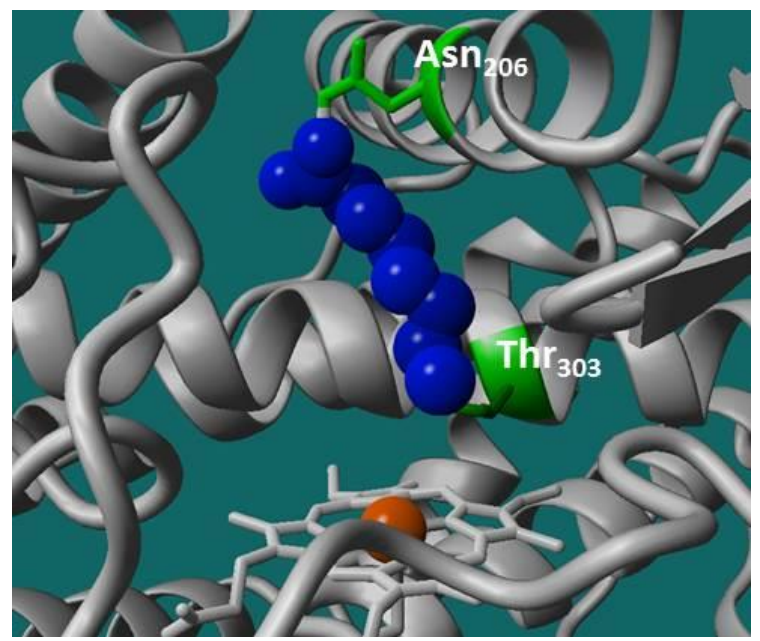

Figure 6. Schematic presentation of the interaction of C8 carboxylic acid with the Asn 206 residue of the CYP2E1 molecule. The $\mathrm{Thr}_{303}$ residue, in the vicinity of the CYP2E1 active site is also shown. 

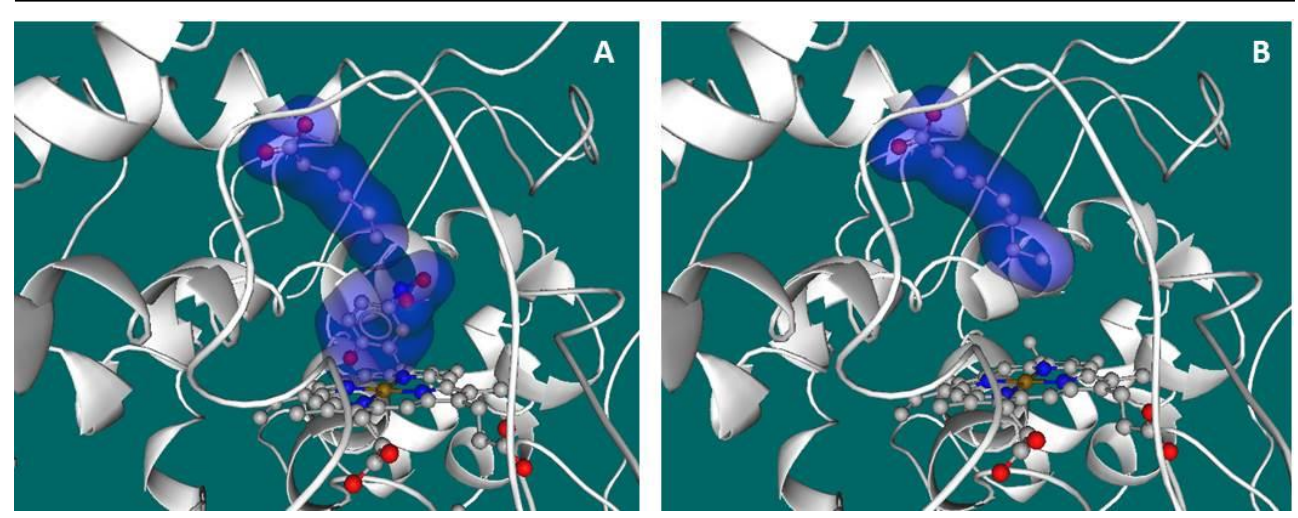

Figure 7. A) Schematic presentation of the interaction of a medium chain carboxylic acid (C6) with the Asn 206 residue of the CYP2E1 molecule, which shows $\mathrm{C} 6$ carboxylic acid does not impede interaction of 4nitrophenol with the CYP2E1 active site. B) Interaction of C8 carboxylic acid with the Asn 206 residue, which does impede interaction of 4-nitrophenol with the CYP2E1 active site.

CYP2E1 inhibitors might block the formation of reactive oxygen species by binding to the CYP2E1 active site. Specific inhibition of CYP2E1 might also prevent the formation of reactive or carcinogenic metabolites. In this way, CYP2E1 inhibitors could play a role in the treatment or prevention of CYP2E1 associated diseases, such as alcoholic and non-alcoholic fatty liver disease. The CYP2E1 active site and access channel are highly lipophilic, explaining the pivotal role of lipophilicity in the interaction of compounds with CYP2E1, which was also found in the present study. This study indicates that the optimal $\log \mathrm{D}_{7.4}$ value for CYP2E1 inhibition is approximately 2.4. In addition, our study shows that the interaction of carboxylate and other charged moieties with the $\mathrm{Thr}^{303}$ residue and the Asn ${ }^{206}$ residue have to be considered. This information opens avenues to select lead compounds for CYP2E1 inhibition on a rational basis. This could be of importance in the search of a possible treatment for CYP2E1 associated diseases, such as ASH and NASH.

\section{Acknowledgement}

We would like to thank prof. dr. M. Ingelman-Sundberg (Karolinska institute) for the provision of CYP2E1 antibodies. 


\section{References}

1. Trabut, J., et al., Influence of liver biopsy on abstinence in alcohol-dependent patients. Alcohol Alcohol, 2008. 43(5): p. 559-563.

2. O'Shea, R., S. Dasarathy, and A. McCullough, Alcoholic liver disease. Am J Gastroenterol, 2010. 105(1): p. 14-32.

3. te Sligte, K., et al., Non-alcoholic steatohepatitis: review of a growing medical problem. Eur J Intern Med, 2004. 15: p. 10-21.

4. $\quad$ Reid, A.E., Nonalcoholic steatohepatitis. Gastroenterol, 2001. 121: p. 710-723.

5. McClain, C.J., et al., Mechanisms of non-alcoholic steatohepatitis. Alcohol, 2004. 34: p. 67-79.

6. Preiss, D. and N. Sattar, Non alcoholic fatty liver disease: an overview of prevalence, diagnosis, pathogenesis and treatment considerations. Clin Sci (Lond), 2008. 115(5): p. 141-150.

7. McCullough, A.J., Pathophysiology of nonalcoholic steatohepatitis. J Clin Gastroenterol, 2006. 40(3 (Suppl 1)): p. S17-29.

8. Koek, G.H., P.R. Liedorp, and A. Bast, The role of oxidative stress in non-alcoholic steatohepatitis. Clin Chim Acta, 2011. 412(15-16): p. 1297-1305.

9. Weltman, M.D., G.C. Farrell, and C. Liddle, Increased hepatocyte CYP2E1 expression in a rat nutritional model of hepatic steatosis with inflammation. Gastroenterology, 1996. 111: p. 1645-1653.

10. Weltman, M.D., et al., Hepatic cytochrome P450 $2 E 1$ is increased in patients with nonalcoholic steatohepatitis. Hepatology, 1998. 27(1): p. 128-133.

11. Lu, Y. and A. Cederbaum, CYP2E1 and oxidative liver injury by alcohol. Free Radic Biol Med, 2008. 44(5): p. 723-738.

12. Lieber, C.S., CYP2E1: from ASH to NASH. Hepatol Res, 2004. 28: p. 1-11.

13. Videla, L., et al., Oxidative stress-related parameters in the liver of non-alcoholic fatty liver disease patients. Clin Sci (Lond), 2004. 106(3): p. 261-268.

14. Orellana, M., et al., Relationship between in vivo CHZ hydroxylation, hepatic cytochrome P450 $2 E 1$ content and liver injury in obese non-alcoholic fatty liver disease patients. Hepatol Res, 2006. 34: p. 57-63.

15. Dupont, I., et al., Cytochrome P4502E1 inducibility and hydroxyethyl radical formation among alcoholics. J Hepatol, 1998. 28(4): p. 564-571.

16. Robertson, G., I. Leclercq, and G.C. Farrell, Nonalcoholic steatosis and steatohepatitis II. Cytochrome p-450 enzymes and oxidative stress. Am J Physiol Gastrointest Liver Physiol, 2001. 281: p. G1135-G1139.

17. Porubsky, P.R., K.M. Meneely, and E.E. Scott, Structures of human cytochrome P-450 2E1. Insights into the binding of inhibitors and both small molecular weight and fatty acid substrates. J Biol Chem, 2008. 238(48): p. 33698-33706.

18. White, R., The involvement of free radicals in the mechanism of monooxygenases. Pharmac Ther, 1991. 49: p. 21-42.

19. Zangar, R., D. Davydov, and S. Verma, Mechanisms that regulate production of reactive oxygen species by cytochrome P450. Toxicol Appl Pharmacol, 2004. 199: p. 316-331. 
20. Bell, L.N., et al., Bariatric surgery-induced weight loss reduces hepatic lipid peroxidation levels and affects hepatic cytochrome P-450 protein content. Annals of surgery, 2010. 251(6): p. 1041-8.

21. Leung, T.M. and N. Nieto, CYP2E1 and oxidant stress in alcoholic and non-alcoholic fatty liver disease. Journal of Hepatology, 2013. 58(2): p. 395-8.

22. Kraner, J., et al., Induction of P4502E1 by acetone in isolated rabbit hepatocytes - role of increased protein and mRNA synthesis. Biochem Pharmacol, 1993. 45: p. 1483-1492.

23. Smith, P.K., et al., Measurement of protein concentration using bicinchoninic acid. Anal Biochem, 1985. 150(1): p. 76-85.

24. Elbarbry, F., K. Wilby, and J. Alcorn, Validation of a HPLC method for the determination of p-nitrophenol hydroxylase activity in rat hepatic microsomes. J Chromatogr, 2006. 834: p. 199-203.

25. Topliss, J.G., Utilization of operational schemes for analog synthesis in drugs design. J Med Chem, 1972. 15(10): p. 1006-1011.

26. Aubert, J., et al., Increased expression of cytochrome P450 $2 E 1$ in nonalcoholic fatty liver disease: mechanisms and pathophysiological role. Clinics and research in hepatology and gastroenterology, 2011. 35(10): p. 630-7.

27. Lewis, D.F., et al., Molecular modelling of human CYP2E1 by homology with the CYP102 haemoprotein domain: investigation of the interactions of substrates and inhibitors within the putative active site of the human CYP2E1 isoform. Xenobiotica; the fate of foreign compounds in biological systems, 2000. 30(1): p. 1-25.

28. Martikainen, L.E., et al., Interactions of inhibitor molecules with the human CYP2E1 enzyme active site. European journal of pharmaceutical sciences : official journal of the European Federation for Pharmaceutical Sciences, 2012. 47(5): p. 996-1005.

29. Lewis, D.F., Quantitative structure-activity relationships in a series of alcohols exhibiting inhibition of cytochrome P-450-mediated aniline hydroxylation. Chem Biol Interact, 1987. 62(3): p. 271-280.

30. Porubsky, P.R., K.P. Battaile, and E.E. Scott, Human cytochrome P450 2 E1 structures with fatty acid analogs reveal a previously unobserved binding mode. The Journal of biological chemistry, 2010. 285(29): p. 22282-90. 
Chapter 7

General discussion 


\section{From pathogenesis to pharmacotherapy}

Up to today, non-alcoholic fatty liver disease (NAFLD) research focuses on identifying the mechanism responsible for the transition from simple steatosis to steatohepatitis resulting in complicated liver and systemic diseases. Initially, this search was based on the hypothesis that the pathogenesis of NAFLD comprised two 'hits': after the first hit of steatosis, a second hit (e.g. oxidative stress) was needed to develop steatohepatitis [1]. This relative simple concept later evolved to the multiple hits pathogenesis [2]. In this theory, multiple parallel hits, derived from white adipose tissue, the gut liver interaction (microbiome) and the liver itself are required to cause progression from steatosis to non-alcoholic steatohepatitis (NASH) in about one third of the patients.

Since more and more 'hits' or pieces of the NAFLD pathogenesis are unraveled and the conceptual idea of the pathogenesis has changed, the approach to search a suitable pharmacotherapy for NAFLD should also change accordingly. If only one of the 'hits' in the pathogenesis is targeted, other pathogenic processes proceed. Therefore, we propose that a pharmacotherapy for NAFLD should target multiple pathogenic mechanisms. The first step is to identify the key factors in the pathways that affect the transition from simple steatosis to steatohepatitis. We identified increased plasma citrate levels in the metabolic pathway and hypothesized that this plays a crucial role in the generation of oxidative stress.

\section{Role of citrate in NAFLD}

We demonstrated that citrate might also be one of the 'hits' in NAFLD, leading to oxidative stress (chapter two). The hypothesis that citrate could contribute to NAFLD, originates from the observation that patients with citrin deficiency develop NAFLD and NASH, without presenting other features of the metabolic syndrome [3]. Citrin deficiency is caused by a mutation in the gene encoding citrin, leading to a deficient mitochondrial aspartate-glutamate carrier. This carrier is part of the malateaspartate shuttle and exchanges mitochondrial aspartate for cytosolic glutamate and a proton. This is important for the translocation of cytosolic nicotinamide dinucleotide (NADH) reducing equivalents into the mitochondria, since NADH cannot pass the mitochondrial membrane itself. Komatsu et al. [3] hypothesized that in response to the deficient aspartate-glutamate carrier, the malate-citrate carrier is upregulated, leading to an increase of citrate in the cytosol. The large amount of citrate is subsequently converted into acetyl-CoA by ATP-citrate lyase. The acetyl-CoA is then 
converted into fatty acids, causing steatosis [3]. Based on the characteristic of citrate that it cannot be oxidized in the human body, we hypothesized that the interaction of citrate with oxidizing enzymes results in "leaking" of these reactive oxygen species from these oxidizing enzymes, and in this way contribute to the progression of NAFLD.

We observed that in patients with NAFLD, without citrin deficiency, citrate levels were elevated (chapter two). This could originate from increased supply of free fatty acids that are broken down into acetyl-CoA during $\beta$-oxidation. The acetyl-CoA is used to form citrate in the first step of the citric acid cycle. Although we could not demonstrate that citrate itself increases oxidative stress, we demonstrated that citrate can contribute to oxidative stress by its function as an iron chelator. As iron chelator, citrate promotes the formation of hydroxyl radicals in the Fenton reaction.

Further research is needed to demonstrate the impact of increased citrate levels on NAFLD. When the role of citrate is established, therapy could be aimed at the reduction of citrate levels or inhibition of citrate induced oxidative stress. Here, flavonoids can come into play, since these bioactives are excellent iron chelators and can compete with citrate for iron chelation [4-6] and in this way reduce citrate induced oxidative stress. Furthermore, by iron chelation, flavonoids can directly scavenge radicals produced in the Fenton reaction by site specific scavenging $[7,8]$. Interestingly, a previous in vivo study, examining the addition of citrate to human erythrocytes, reported that citrate increased lipid peroxidation and decreased antioxidant activity in human erytrocytes, and that this effect was diminished by the flavonoid quercetin [9].

\section{Animal models in pharmacotherapy research}

With the use of animal models in pharmacotherapy research, several factors have to be taken into account before extrapolating the results to the human situation. Firstly, it is most often not possible to imitate the exact human disease process in an animal model. Since the pathogenesis of NAFLD in man is so complex and multifactorial, and also differs between individuals, a suitable animal model that covers all 'hits', does not exist (chapter three). The choice of an animal model for a specific study should be based on the pathogenic mechanisms that will be investigated in that study. Secondly, while the animals used in pharmacotherapy research often form a relatively homogenous population (same age, genetic background, environment, gender, etc.), patient populations are much more heterogeneous. As a result, a pharmacotherapy that is successful in animal trials might fail in clinical trials or only show benefit in a 
relatively small, specific group of patients. Furthermore, differences in the metabolism of compounds between animal and man will affect the efficacy of the compound tested.

In our study investigating the flavonoid monoHER for the prevention of the initiation of inflammation in NAFLD (chapter 5), we used the low density lipoprotein-receptor (LDL-receptor) knock out mouse on a high fat diet, since this model is used to study the onset of inflammation in NAFLD [10]. The LDL-receptor knockout mouse is frequently used to study atherosclerosis, but also proved to show signs of hepatic steatosis and inflammation after 7 days of a high fat high cholesterol diet (HFC) and signs of liver fibrosis after three months of a HFC diet [10]. In addition, other signs of the metabolic syndrome, such as obesity, insulin resistance and dyslipidemia were found in LDL-receptor knockout mice fed a diabetogenic diet rich in fat and carbohydrates [11]. Furthermore, mitochondria from various tissues of LDL-receptor knockout mice, including liver, were demonstrated to generate more reactive oxygen species (ROS) than mitochondria from control wildtype mice [12] and lipid peroxidation was found to be increased in LDL-receptor knockout mice fed a high cholesterol diabetogenic diet [13], indicating the presence of a state of oxidative stress. The LDL-receptor knockout mouse on HFC or on a diabetogenic diet appears to be a good model to study NAFLD, since it shows development of steatosis, steatohepatitis and fibrosis, involving metabolic and inflammatory pathways and oxidative stress, and it displays other signs of the metabolic syndrome. Due to the rapid development of steatosis and steatohepatitis in particular, the LDL-receptor knockout mice seem very useful to investigate a pharmacotherapy aimed at the prevention of the onset of inflammation in NAFLD.

In our study comparing the LDL-receptor knockout mice on a HFC diet with wildtype mice and liver specific superoxide dismutase 2 (SOD2) knockout mice on a HFC diet (chapter 4) we observed indeed an increase in inflammation in the livers of the LDLreceptor knockout mice. However, we did not find a significant increase in oxidative stress in these mice, indicating that the pathway of oxidative stress might be less important in the development of inflammation in these mice when fed a HFC diet. In the same study, we investigated if a liver specific SOD2 knockout mouse on a HFC diet would be a good model to study oxidative stress and the onset of inflammation in NAFLD. In this model no increase in oxidative stress and inflammation was found compared to wildtype mice on a HFC diet. Therefore, this model could not be used to investigate the effect of monoHER on the onset of inflammation. 


\section{Flavonoids in the treatment of disease}

Flavonoids are compounds derived from plants and are present in our everyday diet in fruits, vegetables, nuts, herbs and other food products. Within plants, flavonoids have a role in electron transport during photosynthesis, the protection against prooxidant effects of ultraviolet light and the protection against pathogens such as bacteria and viruses [14]. Over 4,000 different flavonoids have been identified up till now [15]. Most flavonoids consist of two phenyl groups (A and B) linked by a pyrane ring (C) (figure 1). The different flavonoid classes are determined by the connection position of the $\mathrm{B}$ ring and $\mathrm{C}$ ring and the degree of oxidation, hydroxylation and unsaturation of the $\mathrm{C}$ ring. The individual flavonoids within a class are determined by the pattern of substitution of the molecule $[14,15]$.

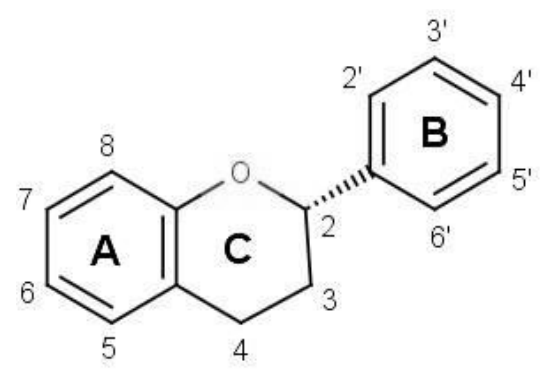

Figure 1. Basic chemical structure of flavonoids.

Health benefits of flavonoids have been reviewed in many studies [16-18]. Initially, observational studies suggested a relationship between the intake of a diet rich in fruits and vegetables and a low prevalence of degenerative diseases, such as cancer and cardiovascular disease [14]. Because in vitro and in vivo studies showed potential health effects of flavonoids, derived from fruits and vegetables, it was hypothesized that the observed relationship was caused by the flavonoid content of the diet. Subsequently, epidemiological studies demonstrated that the intake of a flavonoid rich diet could indeed play a role in the prevention of cardiovascular disease and cancer $[14,19]$.

Several flavonoids, such as epigallocathecin gallate (EGCG), naringenin and quercetin, have been investigated in in vitro and animal studies for the treatment of various diseases, such as diabetes, dementia, inflammatory bowel disease and cardiovascular disease. [20-23]. Although animal studies showed encouraging results of flavonoids in the treatment of these diseases, human studies treating patients or people at risk of 
these diseases are scarce and of short duration [24-27]. Therefore, the results of these studies are inconclusive. Several reasons for the limited research of flavonoids in clinical trials can be pointed out. One is that the focus has been on the flavonoids themselves, while it is increasingly realized that metabolites of flavonoids also play a pivotal role. Furthermore, the modulating effect of flavonoids on the efficacy of drugs and other treatments is still largely unknown. More structure activity relationship studies are needed [20] and can help to elucidate the molecular mechanisms of the effects of flavonoids, which can be used to select the most promising flavonoids.

Flavonoids appear to be ideal candidates to form a multiple target pharmacotherapy for NAFLD, since they affect several of the pathogenic mechanisms related to NAFLD, such as oxidative stress, inflammation, insulin resistance and other metabolic disorders (chapter three). Numerous studies have investigated the use of flavonoids in animal models of NAFLD. Several protective effects of flavonoids against the pathogenic mechanisms of NAFLD were observed in these studies. Clinical trials on the use of flavonoids in NAFLD patients have been performed with silybin (also known as silibinin), either in combination with low dosage of vitamin E (60 IU) [28] or in combination with phosphatidylcholine and a low dosage of vitamin E (45 IU) [29]. Improvement of alanine aminotransferase (ALT), aspartate aminotransferase (AST) and gamma-glutamyl transpeptidase ( $\gamma \mathrm{GT}$ ) levels were found in one study [29], but not in the other [28]. Also positive effects on insulin resistance [29], waist circumference [28] and body mass index (BMI) [28, 29] were found. Indexes of steatosis improved in the study of Sorrenthino et al. [28]. In patients that agreed to liver biopsy, significant improvements in steatosis, inflammation and fibrosis were found in the liver biopsies compared to placebo treated patients in the study of Loguercio et al. [29]. However, since a combination of the flavonoid silybin with other compounds was used, the observed effects cannot be ascribed to the use of the flavonoid solely. Phosphatidylcholine is used to increase the oral bioavailability of silybin, but also has biological effects itself since it is an emulsifier in the lungs, gastrointestinal tract and bile and it is the principal molecular building block for circulating lipoproteins and cell membranes [30]. Previous studies have shown that polyenylphosphatidylcholine, a mixture of polyunsatured phosphatidylcholines, inhibits alcohol induced fibrosis and cirrhosis in baboons and tetrachloride induced fibrosis in rats [31, 32]. Nevertheless, this attenuating effect of polyenylphosphatidylcholines on alcholic liver fibrosis could not be confirmed in humans [33]. The rationale behind the use of silybin combined with vitamin E, in the form of $\alpha$-tocopherol, is not completely clear, since the applied dosage of vitamin $\mathrm{E}$ is 
very low compared to the dosage used in the treatment of NASH (800 IU) [34]. Moreover, the antioxidant activity of the flavonoids epicatechin and EGCG was previously found to be suppressed by addition of $\alpha$-tocopherol in in vitro experiments [35], suggesting that addition of $\alpha$-tocopherol to silybin might also reduce its antioxidant activity. However, synergistic actions between $\alpha$-tocopherol and flavonoids have also been reported [36-38].

A recently published randomized placebo controlled clinical trial in which NAFLD patients received anthocyanins for 12 weeks, showed significant improvements in ALT, Cytokeratin-18 M30 fragment and myeloperoxidase levels, while no significant changes in waist circumference and BMI were found [39].

So far, published in vitro and animal studies demonstrate that flavonoids affect different pathogenic mechanisms that are also involved in the NAFLD pathogenesis. Furthermore, results in animal studies and the first clinical studies on flavonoids, indicate that flavonoids might be good candidates in the search of a pharmacotherapy for NAFLD. More research is needed to establish the effects of flavonoids and flavonoid metabolites in humans and to determine the possible role of flavonoids in the treatment of NAFLD.

\section{MonoHER in the treatment of NAFLD}

The effect of the flavonoid monoHER on inflammation and oxidative stress was studied in an animal model of NAFLD (chapter five). We selected monoHER, because this semisynthetic compound closely resembles the natural flavonoid rutin, but has higher bioavailability. Rutin has shown promising effects against NAFLD in the few animal studies that have been performed so far. It was found to reduce hepatic and/or serum lipid values, such as triglycerides and cholesterol and to reduce weight, insulin resistance, oxidative stress and inflammatory markers. Furthermore, it showed improvement of liver histology regarding steatosis and inflammation (chapter three).

Remarkably, no effect of monoHER on histological signs of steatosis and inflammation was found in LDL-receptor knock out mice on a high fat diet. Also, no significant effect on lipid peroxidation and the glutathione (GSH)/glutathione disulfide (GSSG)-ratio was found. An explanation for these findings could be the low power of the study caused by the relatively large variation seen in the results, and the relatively low level of inflammation that was induced in the untreated group. The relatively low level of inflammation might be caused by the design of the study that aimed at investigating 
the onset of inflammation. At the onset of the disease, the effects are relatively small compared to the biological variation, which might explain why we did not observe a significant effect with the limited number of animals used. Also the choice of animal model might be relevant. Although, the LDL-receptor knockout mouse shows signs of steatosis, inflammation and fibrosis after an extended period of a HFC diet, resembling human NASH, the mechanism of induction of inflammation might be different. The LDL-receptor regulates the plasma cholesterol level by removing intermediate density lipoproteins (IDL) and LDL from the plasma [40]. Once internalized within a cell, the lipoproteins are delivered to the lysosomes, where its cholesteryl esters are hydrolyzed [40]. The cell uses the liberated cholesterol for the synthesis of cell membranes, bile acids and steroids or stores it in the form of cytoplasmic cholesteryl esters droplets [40]. Lack of LDL-receptors in mice leads to a marked increase in plasma IDL and LDL and a small increase in plasma very low density lipoprotein (VLDL) [41]. In the livers of LDL-receptor knockout mice on a high fat high cholesterol diet, lysosomal fat accumulation is found within the Kupffer cells [42]. This is probably caused by the uptake of oxidized LDL-cholesterol and possibly leads to lysosomal dysfunction and an inflammatory response [42]. Therefore, the disease mechanism of NASH in the LDL-receptor mice on HFC diet appears to be less multi-factorial than human NAFLD. It is imaginable that monoHER does not target the lysosomal fat accumulation and the subsequent inflammatory response. Although we previously assumed that the LDL-receptor knockout mouse on a HFC diet could be a good model to investigate a possible pharmacotherapy for human NAFLD, this seems questionable. Up to date, lysosomal fat accumulation in Kupffer cells has not been reported in the livers of NAFLD and NASH patients in the way it is seen in the LDLrecepter knock out mice. However, cholesterol accumulation in Kupffer cells (not specifically in the lysosomes) was reported in $4 \mathrm{NASH}$ patients and in $\mathrm{C} 57 \mathrm{Bl} / 6 \mathrm{~J}$ mice on a HFC diet that developed steatohepatitis, while this was not found in 3 patients with 'simple' steatosis and $\mathrm{C} 57 \mathrm{Bl} / 6 \mathrm{~J}$ mice on a high fat diet that only developed steatosis, suggesting a role for cholesterol loading of Kupffer cells in the initiation of inflammation [43]. Further research should investigate the exact role of cholesterol in the development of NASH and should examine if lysosomal cholesterol accumulation in Kupffer cells also plays a role in human NASH.

Another reason why we could not find an effect of monoHER on signs of steatosis and inflammation could be that we administered monoHER only once a day, while animals were exposed to the HFC diet continuously. Previous studies on monoHER in mice have shown that after subcutaneous administration of monoHER, monoHER could not 
be detected in the plasma for longer than two hours after administration. However, monoHER is longer present in the liver [44] and excreted in bile it could be reabsorbed via the enterohepatic circulation.

Although no direct effects on the development of steatohepatitis were found with the use of monoHER, it was found that the adaptive response to oxidative stress tended to be higher in monoHER treated LDL-receptor knock out mice, evidenced by higher gene expression of heme-oxygenase 1 and glutathione peroxidase 3. Also, a within animal relationship was found, linking a high adaptive response to a low redox status in monoHER treated LDL-receptor knock out mice. This relationship was absent in placebo treated mice and in placebo and monoHER treated wildtype mice. This indicates that monoHER seems to promote the adaptive response when this is needed, in animals with a relative low redox status, and that MonoHER does not promote the adaptive response when this is not needed: when the redox status is high or when oxidative stress is absent.

These findings raise questions for further research, such as: can monoHER also stimulate the adaptive response to oxidative stress in humans, and do other flavonoids also stimulate the adaptive response to oxidative stress in this way? Furthermore, questions remain on the potential of monoHER in the treatment of NAFLD. For example: Can an effect of monoHER on steatohepatitis be found when the disease is more advanced and the disease characteristics are more evident? Or when monoHER is administered more often? Future research should also elucidate the role of flavonoid metabolites and the interaction of flavonoids with other therapies and other components of the diet.

\section{Characteristics of CYP2E1 inhibitors}

Because cytochrome P450 2E1 (CYP2E1) is implicated in the pathogenesis of both alcoholic and non-alcoholic fatty liver disease, we investigated which chemical characteristics of compounds are important for CYP2E1 inhibition (chapter six). CYP2E1 is able to induce liver damage by the production of ROS during its catalytic cycle and by the formation of toxic metabolites, such as acetaldehyde in alcoholic liver disease (ALD) and dicarboxylic fatty acids in NAFLD [45]. The finding that lipophilicity is the major characteristic determining binding to the CYP2E1 active site advances the search for compounds for the treatment of ALD and NAFLD. With these compounds, the role of CYP2E1 can be determined more accurately. 
Regarding the search for a potential pharmacotherapy for NAFLD, it should be realized that CYP2E1 is only one facet of the complex NAFLD pathogenesis. Inhibition of CYP2E1 will probably not stop the progression of the disease. However, compounds that inhibit CYP2E1 and target other components of the NAFLD pathogenesis, warrant further investigation. It is therefore interesting to determine the potential of flavonoids and other bioactives to inhibit the production of reactive metabolites and ROS by CYP2E1.

\section{Overall conclusion}

The research described in this thesis advocates that the search for a pharmacotherapy for NAFLD should focus on the multiple hits that are implicated in the NAFLD pathogenesis. This means that instead of targeting one pathogenic component, a treatment should target multiple components of the NAFLD pathogenesis. In line with the complex pathogenesis with interindividual differences, it seems impossible to capture all of the facets of NAFLD in a single animal model. Therefore, the choice of animal model should depend on the pathogenic mechanism that is investigated in a specific study. In the search of a multiple target treatment for NAFLD, this implies that a treatment should be investigated in various animal models. Finally, we propose flavonoids as potential treatment for NAFLD, since these bioactives affect several mechanisms implicated in the pathogenesis, including oxidative stress as well as metabolic and inflammatory pathways.

\section{Future perspective}

The studies presented in this thesis give several leads for future research. Subjects of interest are:

- The NAFLD pathogenesis and the causes of progression to NASH: investigation if certain subgroups of patients exist, in which a similar complex of factors causes progression to NASH. Subsequently, it could be studied if these subgroups of patients can benefit from the same treatment or combination of treatments. This should lead to a more personalized treatment for patients with NAFLD.

- Citrate levels in NAFLD patients: investigation of plasma and liver citrate levels in a larger group of NAFLD and NASH patients. It should also be explored if increased citrate levels can be linked to liver damage in NASH 
patients. If a relationship between increased citrate levels and liver damage is demonstrated, means to reduce citrate levels should be investigated.

- Similarities in the pathogenesis of NASH in LDL-receptor knockout mice and NASH patients. In particular, the role of lysosomal cholesterol accumulation in Kupffer cells in human NASH and the way to target the resulting cholesterol accumulation with a therapy.

- The contribution of reactive oxygen species produced by CYP2E1 to ALD and NAFLD, as well as means to reduce the oxidative stress induced in this way.

- Further examination of the health effects of flavonoids and flavonoid metabolites in humans and in NAFLD patients. More specifically, the effect of monoHER and other flavonoids, including their metabolites on the adaptive response of the liver to cope with the various hits involved in ALD and NAFLD. 


\section{References}

1. Day, C.P. and O.F.W. James, Steatohepatitis: A Tale of Two "Hits"? Gastroenterology, 1998. 114(4): p. 842-845.

2. Tilg, H. and A.R. Moschen, Evolution of inflammation in nonalcoholic fatty liver disease: the multiple parallel hits hypothesis. Hepatology, 2010. 52(5): p. 1836-46.

3. Komatsu, M., et al., Citrin deficiency as a cause of chronic liver disorder mimicking nonalcoholic fatty liver disease. J Hepatol, 2008. 49: p. 810-820.

4. Pietta, P.G., Flavonoids as antioxidants. Journal of natural products, 2000. 63(7): p. 1035-42.

5. Ross, J.A. and C.M. Kasum, Dietary flavonoids: bioavailability, metabolic effects, and safety. Annual review of nutrition, 2002. 22: p. 19-34.

6. van Acker, S.A., et al., Influence of iron chelation on the antioxidant activity of flavonoids. Biochem Pharmacol, 1998. 56(8): p. 935-43.

7. Haenen, G.R.M.M., F.P. Jansen, and A. Bast, The antioxidant properties of five O- $(\beta$ hydroxyethyl)-rutosides of the flavonoid mixture venoruton. Phlebology, 1993. 8(suppl 1): p. 10-17.

8. Lemmens, K.J., et al., The flavonoid 7-mono-O-(beta-hydroxyethyl)-rutoside is able to protect endothelial cells by a direct antioxidant effect. Toxicol In Vitro, 2014. 28(4): p. 538-43.

9. Bas, H., S. Kalender, and D. Pandir, In vitro effects of quercetin on oxidative stress mediated in human erythrocytes by benzoic acid and citric acid. Folia Biol (Krakow), 2014. 62(1): p. 59-66.

10. Bieghs, V., et al., LDL receptor knock-out mice are a physiological model particularly vulnerable to study the onset of inflammation in non-alcoholic fatty liver disease. PLoS One, 2012. 7(1): p. e30668.

11. Schreyer, S.A., et al., LDL receptor but not apolipoprotein E deficiency increases dietinduced obesity and diabetes in mice. Am J Physiol Endocrinol Metab, 2002. 282(1): p. E207-14.

12. Oliveira, H.C., et al., Oxidative stress in atherosclerosis-prone mouse is due to low antioxidant capacity of mitochondria. Faseb j, 2005. 19(2): p. 278-80.

13. Subramanian, S., et al., Dietary cholesterol exacerbates hepatic steatosis and inflammation in obese LDL receptor-deficient mice. J Lipid Res, 2011. 52(9): p. 1626-35.

14. Graf, B.A., P.E. Milbury, and J.B. Blumberg, Flavonols, flavones, flavanones, and human health: epidemiological evidence. J Med Food, 2005. 8(3): p. 281-90.

15. Middleton, E., Jr., Effect of plant flavonoids on immune and inflammatory cell function. Adv Exp Med Biol, 1998. 439: p. 175-82.

16. Wang, H.K., The therapeutic potential of flavonoids. Expert Opin Investig Drugs, 2000. 9(9): p. 2103-19.

17. Gonzalez, R., et al., Effects of flavonoids and other polyphenols on inflammation. Critical reviews in food science and nutrition, 2011. 51(4): p. 331-62.

18. Nijveldt, R.J., et al., Flavonoids: a review of probable mechanisms of action and potential applications. The American journal of clinical nutrition, 2001. 74(4): p. 418-25.

19. Arts, I.C. and P.C. Hollman, Polyphenols and disease risk in epidemiologic studies. Am J Clin Nutr, 2005. 81(1 Suppl): p. 317s-325s. 
20. Babu, P.V., D. Liu, and E.R. Gilbert, Recent advances in understanding the anti-diabetic actions of dietary flavonoids. J Nutr Biochem, 2013. 24(11): p. 1777-89.

21. Quinones, M., M. Miguel, and A. Aleixandre, Beneficial effects of polyphenols on cardiovascular disease. Pharmacol Res, 2013. 68(1): p. 125-31.

22. Somani, S.J., et al., Phytochemicals and their potential usefulness in inflammatory bowel disease. Phytother Res, 2015. 29(3): p. 339-50.

23. Orhan, I.E., et al., Flavonoids and dementia: an update. Curr Med Chem, 2015. 22(8): p. 1004-15.

24. Li, D., et al., Purified anthocyanin supplementation reduces dyslipidemia, enhances antioxidant capacity, and prevents insulin resistance in diabetic patients. J Nutr, 2015. 145(4): p. 742-8.

25. Ebrahimpour Koujan, S., et al., Effects of Silybum marianum (L.) Gaertn. (silymarin) extract supplementation on antioxidant status and hs-CRP in patients with type 2 diabetes mellitus: a randomized, triple-blind, placebo-controlled clinical trial. Phytomedicine, 2015. 22(2): p. 290-6.

26. Schar, M.Y., et al., Orange juice-derived flavanone and phenolic metabolites do not acutely affect cardiovascular risk biomarkers: a randomized, placebo-controlled, crossover trial in men at moderate risk of cardiovascular disease. Am J Clin Nutr, 2015. 101(5): p. 931-8.

27. Dryden, G.W., et al., A pilot study to evaluate the safety and efficacy of an oral dose of (-)epigallocatechin-3-gallate-rich polyphenon $E$ in patients with mild to moderate ulcerative colitis. Inflamm Bowel Dis, 2013. 19(9): p. 1904-12.

28. Sorrentino, G., et al., Efficacy of lifestyle changes in subjects with non-alcoholic liver steatosis and metabolic syndrome may be improved with an antioxidant nutraceutical: a controlled clinical study. Drugs R D, 2015.15(1): p. 21-5.

29. Loguercio, C., et al., Silybin combined with phosphatidylcholine and vitamin $E$ in patients with nonalcoholic fatty liver disease: a randomized controlled trial. Free radical biology \& medicine, 2012. 52(9): p. 1658-65.

30. Kidd, P.M., Bioavailability and activity of phytosome complexes from botanical polyphenols: the silymarin, curcumin, green tea, and grape seed extracts. Altern Med Rev, 2009. 14(3): p. 226-46.

31. Lieber, C.S., et al., Phosphatidylcholine protects against fibrosis and cirrhosis in the baboon. Gastroenterology, 1994. 106(1): p. 152-9.

32. Ma, X., J. Zhao, and C.S. Lieber, Polyenylphosphatidylcholine attenuates non-alcoholic hepatic fibrosis and accelerates its regression. J Hepatol, 1996. 24(5): p. 604-13.

33. Lieber, C.S., et al., II. Veterans Affairs Cooperative Study of polyenylphosphatidylcholine in alcoholic liver disease. Alcohol Clin Exp Res, 2003. 27(11): p. 1765-72.

34. Chalasani, N., et al., The diagnosis and management of non-alcoholic fatty liver disease: practice guideline by the American Gastroenterological Association, American Association for the Study of Liver Diseases, and American College of Gastroenterology. Gastroenterology, 2012. 142(7): p. 1592-609.

35. Kadoma, Y., et al., Free radical interaction between vitamin E (alpha-, beta-, gammaand delta-tocopherol), ascorbate and flavonoids. In Vivo, 2006. 20(6b): p. 823-7.

36. Chen, C.Y., et al., Flavonoids from almond skins are bioavailable and act synergistically with vitamins $C$ and $E$ to enhance hamster and human $L D L$ resistance to oxidation. J Nutr, 2005. 135(6): p. 1366-73. 
37. Fujisawa, S., et al., A quantitative approach to the free radical interaction between alpha-tocopherol or ascorbate and flavonoids. In Vivo, 2006. 20(4): p. 445-52.

38. Chuang, C.H., C.S. Huang, and M.L. Hu, Vitamin E and rutin synergistically inhibit expression of vascular endothelial growth factor through down-regulation of binding activity of activator protein-1 in human promyelocytic leukemia (HL-60) cells. Chem Biol Interact, 2010. 183(3): p. 434-41.

39. Zhang, P.W., et al., A CONSORT-compliant, randomized, double-blind, placebo-controlled pilot trial of purified anthocyanin in patients with nonalcoholic fatty liver disease. Medicine (Baltimore), 2015. 94(20): p. e758.

40. Brown, M.S. and J.L. Goldstein, A receptor-mediated pathway for cholesterol homeostasis. Science, 1986. 232(4746): p. 34-47.

41. Ishibashi, S., et al., Hypercholesterolemia in low density lipoprotein receptor knockout mice and its reversal by adenovirus-mediated gene delivery. J Clin Invest, 1993. 92(2): p. 883-93.

42. Bieghs, V., et al., Internalization of modified lipids by CD36 and SR-A leads to hepatic inflammation and lysosomal cholesterol storage in Kupffer cells. PLoS One, 2012. 7(3): p. e34378.

43. Ioannou, G.N., et al., Hepatic cholesterol crystals and crown-like structures distinguish NASH from simple steatosis. J Lipid Res, 2013. 54(5): p. 1326-34.

44. Abou El Hassan, M.A., et al., Bioavailability and pharmacokinetics of the cardioprotecting flavonoid 7-monohydroxyethylrutoside in mice. Cancer Chemother Pharmacol, 2003. 52(5): p. 371-6.

45. Leung, T.M. and N. Nieto, CYP2E1 and oxidant stress in alcoholic and non-alcoholic fatty liver disease. J Hepatol, 2013. 58(2): p. 395-8. 
Chapter 8

Summary 
The main objective of the studies presented in this thesis was to further elucidate the pathogenesis of non-alcoholic fatty liver disease (NAFLD) with an emphasis on the processes of oxidative stress and the onset of inflammation and to find new treatment strategies that target the different pathogenic pathways in NAFLD.

\section{Pathogenesis of NAFLD}

The pathogenesis of NAFLD was first regarded to implicate two hits. Steatosis was considered to be the first hit, which would make the liver vulnerable for a second hit that would subsequently induce inflammation. Nowadays, the pathogenesis is regarded as a multiple hits disease (described in chapter one). Multiple parallel hits, including metabolic abnormalities, inflammatory pathways and oxidative stress, affect the liver and eventually cause non-alcoholic steatohepatitis (NASH). Although the pathogenesis of NAFLD and NASH is increasingly revealed, there is not yet a complete image of all involved processes and new possible pathogenic mechanisms are continually added.

One of the mechanisms we added is based on the elevated plasma citrate levels we observed in NAFLD patients. In chapter two we hypothesized that elevated citrate levels increase oxidative stress in NAFLD patients. Elevated citrate levels could originate from an excessive supply of fatty acids to the liver. Fatty acids, as well as glucose, are broken down into acetyl-CoA. Acetyl-CoA subsequently enters the Krebs cycle, or citric acid cycle, in which it is further processed. In the first step of the Krebs cycle, citric acid is formed from acetyl-CoA and oxaloacetate. In patients with the metabolic syndrome and NAFLD, the influx of acetyl-CoA into the Krebs cycle is expected to be increased due to high levels of free fatty acids and glucose. Because the third step in the Krebs cycle is rate-limiting and the second step is reversible, the high influx of acetyl-CoA will result in the accumulation of citrate. We confirmed that a high supply of fatty acids (palmitic acid) to HepG2 cells leads to increased citrate levels in the cytosol of these cells. Furthermore, we observed that citrate alone, does not increase oxidative stress in HepG2 cells, but citrate combined with hydrogen peroxide further aggravates oxidative stress caused by hydrogen peroxide. This is probably related to the promotion of iron mediated hydroxyl radical formation from hydrogen peroxide by citrate. Interestingly, hepatic iron levels are often elevated in NAFLD patients. Elevated citrate levels in combination with high hepatic iron levels can augment oxidative stress and might contribute to the progression of steatosis into NASH. 


\section{Flavonoids in the treatment of NAFLD}

Traditionally, drugs are developed to act on one target and in this way lead to absence of disease. Because NAFLD is such a multi-factorial disease, affecting one target, will most likely not treat the disease. This might be an explanation why many examined pharmacotherapies for NAFLD and NASH have failed. We propose that a drug used in the treatment of NAFLD and NASH should affect more targets. Therefore, we further investigated flavonoids, antioxidants that also have anti-inflammatory and metabolic effects.

In chapter three we reviewed the biological effects of flavonoids and examined how they could affect the different pathogenic mechanisms of NAFLD. Metabolic effects of flavonoids include stimulation of peroxisome proliferator activated receptors $\alpha$ and $\gamma$ (PPAR $\alpha$ and PPAR $\gamma$ ), inhibition of sterol regulatory binding protein 1c (SREBP-1c) and liver $\mathrm{X}$ receptor $\alpha(\mathrm{LXR} \alpha)$ and stimulation of $5^{\prime}$ adenosine monophosphate-activated protein kinase (AMPK), which lead to an increase in fatty acid oxidation and to a decrease in insulin resistance and lipogenesis. Antioxidant effects of flavonoids comprise the inhibition of the production of reactive oxygen species (ROS) by inhibition of pro-oxidant enzymes and by influencing the metabolic and inflammatory processes that produce ROS. Furthermore, flavonoids are excellent scavengers of ROS and can chelate iron and other transition metals that contribute to the production of ROS, to cause so called 'site-specific scavenging'. Finally, flavonoids can enhance the endogenous antioxidant defense by stimulation of nuclear factor erythroid derived 2 (NRF2). Anti-inflammatory effects of flavonoids include inhibition of nuclear factor $\kappa B$ $(\mathrm{NF}-\mathrm{KB})$, inhibition of the production of cytokines and prostanoids and a decrease of the expression of adhesion molecules. The effects of flavonoids on NAFLD in animal models were also reviewed in chapter three. Silybin, silymarin, green tea flavonoids, soy isoflavones, quercetin and rutin are the most investigated flavonoids or flavonoid mixtures and were found to have positive effects in animal models of NAFLD. A silybin-phophatidylcholine complex was also investigated in patients with NAFLD and demonstrated positive effects on markers of hepatic damage, insulin resistance, body mass index and liver histology (only investigated in a part of the participants).

\section{Animal models}

Animal models that are used to study the effect of flavonoids were evaluated in chapter three. It was concluded that from the investigated studies, the models using 
rats/gerbils on a high fat diet or on a high fat diet combined with high fructose or carbohydrates seem to approximate the conditions of human NAFLD best. Also studies using genetic models combined with a high fat or a methionine choline deficient (MCD) diet can be useful. However, the ideal animal model of NAFLD that mimics liver damage seen in NAFLD patients including steatosis, inflammation and fibrosis, involves the most important pathogenic pathways (metabolic abnormalities, inflammation and oxidative stress) and demonstrates other signs of the metabolic syndrome, has not yet been developed. In chapter four, we investigated if a C57B16/J mouse with a liver specific superoxide dismutase 2 (SOD2) knock out on a high fat high cholesterol diet can be used as a model to study the onset of inflammation in NAFLD. The mice were compared with wildtype mice and LDL-receptor knock out mice on a high fat high cholesterol diet. Interestingly, the mice with a liver specific SOD knock out did not develop inflammation after three weeks of high fat diet, in contrast to the LDL-receptor knock out mice that did develop inflammation after three weeks of high fat diet.

\section{New treatment strategies for NAFLD}

\section{Effect of the flavonoid monoHER in a mouse model of NAFLD}

The LDL-receptor knock out mice on a high fat high cholesterol diet were used to study the effect of the flavonoid monoHER on the onset of inflammation in NAFLD in chapter five. It was found that although daily treatment with monoHER did not prevent the development of inflammation in these mice, the redox status as well as the adaptive response to oxidative stress tended to be higher in monoHER treated mice, although this did not reach significance on a group level. Interestingly, a within animal relationship was found between a high adaptive response (evidenced by a higher hemoxygenase-1 and glutathione peroxidase 3 expression, indicating NRF2 activation) and a low redox status (evidenced by a lower GSH/GSSG-ratio) in monoHER treated LDL-receptor knock out mice. This relationship was lacking in placebo treated LDL-receptor knock out mice and in placebo and monoHER treated wildtype mice. This demonstrates that monoHER causes a relatively potent adaptation: in mice with a low redox status, monoHER stimulates NRF2 activation, while in mice with a high redox status, no evidence for further stimulation by monoHER was seen, indicating that in the animals that have already adapted, no further adaptation is needed. 


\section{Inhibition of cytochrome P450 2E1}

In chapter six we examined which chemical characteristics of compounds are important for cytochrome P450 2E1 (CYP2E1) inhibition. Since CYP2E1 expression and activity appear to be related to liver damage in NASH patients, but also in patients with alcoholic steatohepatitis (ASH), inhibition of CYP2E1 might hinder the occurrence of these diseases. We observed that lipophilicity is the most important characteristic determining CYP2E1 inhibition. Furthermore, in the case of carboxylic acids or other compounds with charged moieties, interactions with polar residues lining the CYP2E1 active site also have to be considered.

Chapter seven discusses the major findings of this thesis and gives perspectives for future research. 

Nederlandse samenvatting 
De belangrijkste doelen van de artikelen uit dit proefschrift zijn om de pathogenese van niet alcoholische vetleverziekte (NAFLD) verder te verhelderen, met een nadruk op de processen van oxidatieve stress en het begin van ontsteking, en om nieuwe behandelstrategieën te vinden die aangrijpen op de verschillende pathogenetische mechanismen van NAFLD.

\section{Pathogenese van NAFLD}

Eerder werd gedacht dat de pathogenese van NAFLD twee aanvallen of 'hits' tegen de lever omvatte. Leververvetting (steatose) werd gezien als de eerste hit, wat de lever kwetsbaar maakte voor een tweede hit die vervolgens tot ontsteking zou leiden. Tegenwoordig wordt NAFLD steeds meer beschouwd als een ziekte waarbij meerdere hits betrokken zijn (hoofdstuk een). Multipele, tegelijk optredende hits, die metabole veranderingen, inflammatoire mechanismen en oxidatieve stress omvatten, treffen de lever en veroorzaken uiteindelijk niet alcoholische vette leverontsteking (NASH). Hoewel er steeds meer bekend wordt over de pathogenese van NAFLD en NASH, hebben we nog geen compleet overzicht van alle betrokken processen en worden er nog altijd nieuwe mogelijke pathogenetische mechanismen toegevoegd.

Een van de mechanismen die wij hebben toegevoegd is gebaseerd op de vondst van verhoogde citraat concentraties in het plasma van NAFLD-patiënten. In hoofdstuk twee, hypothetiseerden wij dat verhoogde citraat concentraties oxidatieve stress vergroten in NAFLD-patiënten. Verhoogde citaat concentraties kunnen voortkomen uit een overvloedige aanvoer van vetzuren naar de lever. Vetzuren worden net als glucose afgebroken tot acetyl-CoA. Acetyl-CoA wordt vervolgens verder verwerkt in de citroenzuurcyclus. In de eerste stap van de citroenzuurcyclus wordt citraat gevormd uit acetyl-CoA en oxaloacetaat. Bij patiënten met het metabole syndroom en NAFLD wordt verwacht dat de toevoer van acetyl-CoA in de citroenzuurcyclus verhoogd wordt door hoge glucose en vrije vetzuren concentraties. Omdat de derde stap in de citroenzuurcyclus snelheid beperkend is en omdat de tweede stap reversibel is, zal een hoge toevoer van acetyl-CoA naar de citroenzuurcyclus leiden tot accumulatie van citraat. Wij bevestigden dat een hoge toevoer van vetzuren (palmitaat) naar HepG2 cellen leidt tot verhoogde citraat concentraties in het cytosol van deze cellen. Daarnaast observeerden we dat citraat op zichzelf oxidatieve stress in HepG2 cellen niet vergroot, maar citraat gecombineerd met waterstofperoxide de oxidatieve stress veroorzaakt door waterstofperoxide verder verergerd. Dit is waarschijnlijk gerelateerd aan stimulatie van de ijzer-gemedieerde vorming van 
hydroxylradicalen uit waterstofperoxide door citraat. Een interessant gegeven is dat ijzer concentraties vaak verhoogd zijn in de levers van NAFLD-patiënten. Verhoogde citraat concentraties zouden in combinatie met hoge ijzer concentraties oxidatieve stress kunnen verergeren en zo kunnen bijdragen aan de progressie van steatose naar NASH.

\section{Flavonoïden in de behandeling van NAFLD}

Traditioneel gezien worden medicijnen ontwikkeld om op één aangrijpingspunt in te werken en zo te leiden tot afwezigheid van ziekte. Omdat NAFLD zo'n multifactoriële ziekte is, zal inwerking op één aangrijpingspunt de ziekte waarschijnlijk niet genezen. Dit zou een verklaring kunnen zijn voor het feit dat veel van de onderzochte farmacotherapieën voor NAFLD en NASH tot op heden gefaald hebben. Wij stellen voor dat een medicijn dat gebruikt wordt voor de behandeling van NAFLD en NASH meerdere aangrijpingspunten moet hebben. Daarom hebben wij flavonoïden verder onderzocht. Dit zijn antioxidanten die tevens anti-inflammatoire en metabole effecten hebben.

In hoofdstuk drie hebben wij een overzicht gemaakt van de biologische effecten van flavonoïden en hebben we beschreven hoe deze effecten de verschillende pathogenetische mechanismen van NAFLD kunnen beïnvloeden. Metabole effecten van flavonoïden omvatten o.a. stimulatie van peroxisoom proliferator geactiveerde receptoren $\alpha$ en $\gamma$ (PPAR $\alpha$ en PPAR $\gamma$ ), remming van sterol regulatory binding protein 1c (SRBP-1c) en lever X receptor $\alpha$ (LXR $\alpha$ ) en stimulatie van $5^{\prime}$ adenosine monophosphate-activated protein kinase (AMPK), welke leiden tot een toename van vetzuur oxidatie en een afname van insuline resistentie en lipogenese. Antioxidant effecten van flavonoïden omvatten o.a. de remming van de productie van vrije zuurstofradicalen (ROS) door remming van pro-oxidante enzymen en door het beïnvloeden van metabole en inflammatoire processen die ROS produceren. Daarnaast zijn flavonoïden uitstekende ROS-wegvangers (scavengers) en kunnen flavonoïden ijzer en andere transitiemetalen die bijdragen aan de productie van ROS cheleren en zo 'site-specific scavenging' uitoefenen. Ten slotte kunnen flavonoïden de endogene antioxidant verdediging verbeteren door stimulatie van nuclear factor erythroid derived 2 (NRF2). Anti-inflammatoire effecten van flavonoïden zijn o.a. remming van nuclear factor $\kappa \mathrm{B}(\mathrm{NF}-\kappa \mathrm{B})$, remming van de cytokine en prostanoïd productie en vermindering van de expressie van adhesie moleculen. De effecten van flavonoïden op NAFLD in proefdiermodellen werden ook beoordeeld in hoofdstuk 
drie. Silybine, silymarine, groene thee flavonoïden, soja isoflavonen, quercetine en rutine zijn de meest onderzochte flavonoïden of flavonoïd mengsels. Deze flavonoïden lieten positieve effecten zien in proefdiermodellen van NAFLD. Een silybinephosphatidylcholine complex werd ook onderzocht in NAFLD-patiënten en veroorzaakte verbetering van markers van leverschade, insuline resistentie, body mass index en lever histologie (de laatste werd alleen onderzocht in een deel van de deelnemers).

\section{Proefdiermodellen}

De proefdiermodellen die gebruikt worden om de effecten van flavonoïden te onderzoeken werden beoordeeld in hoofdstuk drie. Hieruit werd geconcludeerd dat, van de onderzochte studies, de studies die ratten of gerbils met een hoogvet dieet met of zonder hoge concentraties fructose en/of koolhydraten gebruikten, humane NAFLD het beste benaderden. Ook studies die genetische modellen gecombineerd met een hoogvet dieet of een methionine choline deficiënt dieet gebruiken, kunnen nuttig zijn. Het ideale NAFLD-proefdiermodel dat de leverschade die gezien wordt in NAFLDpatiënten (steatose, inflammatie en fibrose) nabootst, waarbij de belangrijkste pathogenetische mechanismen (metabole afwijkingen, inflammatie en oxidatieve stress) betrokken zijn en waarbij ook andere kenmerken van het metabole syndroom tot uiting komen, is echter nog niet ontwikkeld. In hoofdstuk vier onderzochten wij of een C57Bl6/J-muis met een uitschakeling (knock-out) van superoxide dismutase 2 (SOD2) in de lever op een hoogvet dieet rijk in cholesterol, gebruikt kan worden als proefdiermodel om het begin van inflammatie in NAFLD te onderzoeken. De muizen werden vergeleken met wildtype muizen en muizen met een LDL-receptor knock-out op een hoogvet dieet rijk in cholesterol. De muizen met een SOD2 knock-out in de lever ontwikkelden echter geen inflammatie na drie weken van een hoogvet dieet, in tegenstelling tot de muizen met een LDL-receptor knock-out.

\section{Nieuwe behandelstrategieën voor NAFLD}

\section{Effect van de flavonoïd monoHER in een muismodel van NAFLD}

De muizen met een LDL-receptor knock-out op een hoogvet dieet rijk in cholesterol werden gebruikt om het effect van de flavonoïd monoHER op het begin van inflammatie in NAFLD te bestuderen in hoofdstuk vijf. Hoewel dagelijkse behandeling met monoHER de ontwikkeling van ontsteking in deze muizen niet kon 
voorkomen, neigde behandeling met monoHER wel de redox status en de adaptatie op oxidatieve stress te vergroten. Dit was echter niet significant op groepsniveau. Binnen de afzonderlijke met monoHER behandelde LDL-receptor knock-out muizen werd een relatie gevonden tussen een hoge adaptatie op oxidatieve stress (aangetoond door een hogere genexpressie van hemoxygenase- 1 en glutathion peroxidase 3 , wat wijst op NRF2 activatie) en een lage redox status (aangetoond door een lagere GSH/GSSGratio). Deze relatie werd niet gevonden bij met placebo behandelde LDL-receptor knock-out muizen en bij met monoHER of placebo behandelde wildtype muizen. Dit toont aan dat monoHER en relatief effectieve adaptatie veroorzaakt: monoHER stimuleert NRF2 activatie in muizen met een lage redox status, terwijl er geen bewijs wordt gevonden voor verdere stimulatie in muizen die al een hoge redox status hebben. In dieren die zich al aangepast hebben aan hoge niveaus van oxidatieve stress, lijkt geen verdere adaptatie nodig te zijn.

\section{Remming van cytochroom P450 2E1}

In hoofdstuk zes hebben we onderzocht welke chemische eigenschappen van stoffen belangrijk zijn voor inhibitie van cytochroom P450 2E1 (CYP2E1). Omdat CYP2E1 expressie en activiteit gerelateerd lijken te zijn aan lever schade bij NASH-patiënten, maar ook bij patiënten met alcoholische steatohepatitis (ASH), zou remming van CYP2E1 het optreden van deze ziekten kunnen verhinderen. Wij observeerden dat lipofiliciteit de belangrijkste eigenschap is die CYP2E1 inhibitie bepaald. Daarnaast moet er, in het geval van carbonzuren of andere stoffen met geladen groepen, rekening worden gehouden met interacties met polaire residuen rondom de active site van CYP2E1.

In hoofdstuk zeven worden de belangrijkste bevindingen van dit proefschrift besproken en worden vooruitzichten gegeven voor toekomstig onderzoek. 



\section{Valorisatie addendum}




\section{Relevantie}

Non-alcoholic fatty liver disease (NAFLD), in het Nederlands niet-alcoholische vetleverziekte, is wereldwijd de meest voorkomende leverziekte en heeft een prevalentie van 20-30\% in Westerse landen [1, 2]. Aangezien NAFLD vaak gezien wordt bij patiënten met overgewicht, obesitas en diabetes, wordt verwacht dat de prevalentie, evenals de prevalentie van overgewicht, obesitas en diabetes, de komende jaren verder toe zal nemen. Twintig tot dertig procent van de patiënten met NAFLD ontwikkelt niet-alcoholische steatohepatitis (NASH), fibrose, cirrhose en in sommige gevallen hepetocellulair carcinoom. Steeds meer patiënten ontwikkelen een dermate ernstige leverschade dat ze aangewezen zijn op een levertransplantatie. Van het aantal patiënten dat een levertransplantatie krijgt, neemt het aandeel van NAFLD/NASH patiënten toe. In 2009 kreeg in de Verenigde Staten 9,7\% van de patiënten met een levertransplantatie een nieuwe lever i.v.m. NASH [3]. Op dit moment is NASH op hepatitis C na zelfs de meest voorkomende ziekte onder mensen die op de wachtlijst staan voor een levertransplantatie in de Verenigde Staten [4]. Er is sprake van een verhoogde mortaliteit onder NAFLD patiënten t.o.v. de algemene populatie t.g.v. sterfte aan hart- en vaatziekten en kanker en door lever-gerelateerde sterfte. In Nederland werd in 2011 voor 92 miljoen euro uitgegeven aan zorgkosten gerelateerd aan leverziekten, waaronder NAFLD [5]. Ook hierin is een stijging zichtbaar t.o.v. 2007 (73 miljoen euro) en 2003 (54 miljoen euro) [5].

De kennis rondom de pathogenese van NAFLD is groeiende. Het is duidelijk dat bij de pathogenese veel verschillende processen betrokken zijn. Het beeld is echter nog niet compleet en er worden nog steeds nieuwe factoren gevonden die bijdragen aan de ontwikkeling en progressie van NAFLD. Hoewel er al veel onderzoek verricht is naar het zoeken van een medicamenteuze behandeling voor NAFLD, is er tot op heden nog geen medicamenteuze behandeling geregistreerd. Het onderzoek dat gepresenteerd wordt in deze thesis draagt bij aan een verder begrip van de pathogenese van NAFLD en geeft nieuwe inzichten die bijdragen aan de ontwikkeling van medicamenteuze therapieën.

\section{Doelgroepen}

De onderzoeksresultaten uit deze thesis zijn vooral van belang voor patiënten met NAFLD. Tot op heden worden leefstijl interventies zoals een gezond dieet en meer beweging als belangrijkste behandeling gezien. Het is voor veel mensen echter 
moeilijk om zich voor langere tijd aan de voorgestelde levensveranderingen te houden, omdat het veranderen van een gewoonte zeer complex is. Uit studies die leefstijl interventies onderzocht hebben, blijkt compliantie een groot probleem te zijn [6]. Een medicamenteuze behandeling die toegevoegd wordt aan een behandeling met leefstijl interventies zou ondersteuning kunnen bieden om ontsteking en de gevolgen voor het lichaam te behandelen zodat complicaties als hart en vaatziekten, diabetes en kanker teruggedrongen kunnen worden.

\section{Activiteiten/producten}

Hoewel de onderzoeksresultaten uit deze thesis niet direct tot een medicamenteuze behandeling voor NAFLD leiden, worden wel stappen gezet om een gerichte therapie te vinden. Inzicht in pathofysiologische processen vormt de basis voor een beter begrip van het ontstaansmechanisme, de factoren die ziekte verergeren en aangrijpingspunten voor diagnostiek en therapie.

Er zijn op het moment verschillende voedingssupplementen verkrijgbaar die claimen de werking van de lever te ondersteunen (fig. 1). In veel van deze supplementen zit onder andere mariadistel, een bron van de flavonoïd silymarine. Ook groene thee concentraat, met daarin de flavonoïd epigallocatechine gallaat wordt in een aantal supplementen verwerkt. Deze supplementen bestaan echter uit allerlei mengsels met een scala aan stoffen waarbij er geen rekening wordt gehouden met interacties tussen de verschillende stoffen. Ook is niet duidelijk is hoeveel van elke stof wordt opgenomen in het maag-darmkanaal. Wanneer er in toekomstig onderzoek één flavonoïd of meerdere flavonoïden naar voren komen die werkzaam zijn tegen de progressie van NAFLD, zullen deze in het beste geval verwerkt moeten worden in een tablet of capsule. Bij het mogelijke voorschrijven van een flavonoid dient rekening te worden gehouden met het dieet van de patiënt en de actieve stoffen die hierin voorkomen. 


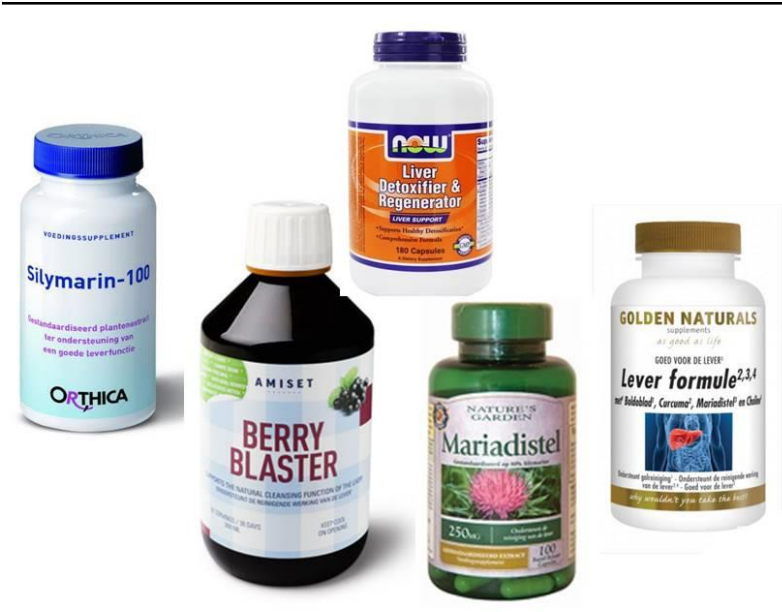

Figuur 1. Voedingssupplementen met o.a. flavonoïden die claimen de werking van de lever te ondersteunen.

\section{Innovatie}

In de studies die beschreven zijn in dit proefschrift zijn verschillende nieuwe hypothesen onderzocht. Verhoogde citraat waarden werden nog niet eerder beschreven in NAFLD patiënten. Ook werd citraat in eerder onderzoek niet gerelateerd aan de pathogenese van NAFLD.

Tevens werd niet eerder onderzocht of SOD2 flox/flox/Alb-Cre muizen met een lever specifieke SOD2 knockout een goed proefdiermodel zouden kunnen zijn om het begin van ontsteking in NAFLD te onderzoeken.

Hoewel verschillende flavonoïden al eerder onderzocht werden in proefdiermodellen van NAFLD, werd nog niet eerder een duidelijk overzicht gegeven van de pathogenetische processen in NAFLD waarop flavonoïden aangrijpen. In dit proefschrift wordt voor het eerst het effect van de flavonoïd MonoHER onderzocht in een proefdiermodel van NAFLD.

\section{Planning}

De onderzoeken die zijn beschreven in dit proefschrift gegenereren verschillende nieuwe hypothesen die het fundament vormen voor gericht vervolgonderzoek. Met dit vervolgonderzoek kan de pathogenese van NAFLD verder worden uitgediept. Een belangrijk aspect is om na te gaan of er, gezien de heterogene patiëntgroep, 
subgroepen van patiënten zijn waarin een specifiek complex aan factoren de progressie van NAFLD naar NASH veroorzaakt. Wanneer zo'n specifiek complex geïdentificeerd is, is de volgende stap om te onderzoeken welke specifieke behandeling die aangrijpt op de betrokken factoren geschikt is voor die subgroep. Ook de rol van citraat in NAFLD en de progressie naar NASH dient onder de loep genomen te worden. De eerste stap hierin is om in een grote patiëntgroep te onderzoeken of citraat spiegels inderdaad gerelateerd zijn aan de ernst van leverschade bij NASH.

Op het gebied van proefdiermodellen zou nog verder onderzoek gedaan kunnen worden, in het bijzonder naar eventuele overeenkomsten in de pathogenese van NASH bij de LDL-receptor knockout muizen en NASH patiënten.

Voor de behandeling van NAFLD verdienen flavonoïden, en vooral MonoHER, nader onderzoek. Naast de flavonoïden zelf dienen ook hun metabolieten in dit onderzoek te worden meegenomen. Met name het effect van deze verbindingen op de adaptieve respons verdient nader onderzoek. 


\section{Verwijzingen}

1. Bedogni, G., et al., Prevalence of and risk factors for nonalcoholic fatty liver disease: the Dionysos nutrition and liver study. Hepatology, 2005. 42(1): p. 44-52.

2. Browning, J.D., et al., Prevalence of hepatic steatosis in an urban population in the United States: impact of ethnicity. Hepatology, 2004. 40(6): p. 1387-95.

3. Charlton, M.R., et al., Frequency and outcomes of liver transplantation for nonalcoholic steatohepatitis in the United States. Gastroenterology, 2011. 141(4): p. 1249-53.

4. Wong, R.J., et al., Nonalcoholic steatohepatitis is the second leading etiology of liver disease among adults awaiting liver transplantation in the United States. Gastroenterology, 2015. 148(3): p. 547-55.

5. RIVM. Cijfertool kosten van ziekten. 2011 [cited 2016 4-10]; Available from: https://kostenvanziektentool.volksgezondheidenzorg.info/tool/nederlands/.

6. Fappa, E., et al., Lifestyle intervention in the management of metabolic syndrome: could we improve adherence issues? Nutrition, 2008. 24(3): p. 286-91. 
Dankwoord 
Ik heb bij de totstandkoming van dit proefschrift hulp gekregen van een groot aantal mensen die ik in dit stukje wil bedanken.

Allereerst wil ik mijn promotieteam bedanken: Aalt Bast, Guido Haenen en Ger Koek. Jullie hebben me tijdens mijn wetenschappelijke stage op de afdeling toxicologie geënthousiasmeerd voor het onderzoek en hebben me na mijn stage de kans geboden om het onderzoek te vervolgen in een promotietraject. Bedankt voor jullie vertrouwen. Daarnaast bedankt voor alle gezellige werkbesprekingen. Als iemand met een geneeskunde achtergrond moest ik regelmatig uitleg krijgen over de chemische termen die voorblij vlogen, gelukkig namen jullie steeds de tijd om me dit weer uit te leggen. Aalt, bedankt voor het mogelijk maken van het promotietraject. Guido, bedankt voor het snelle nakijken en verbeteren van alle teksten die ik je stuurde en het altijd open staan voor alle vragen. Daarnaast bedankt voor je hulp met de muizenstudie en je hulp om Kristien en mij onze angst voor de muizen te laten overwinnen. Ger, bedankt voor de aansporingen die ik, hoewel ze me in stressvolle periodes ook wel konden frustreren, met name aan het einde van het traject toch wel nodig leek te hebben.

Ook wil ik alle leden van de beoordelingscommissie bedanken voor het beoordelen van mijn proefschrift.

Daarnaast wil ik alle collega's van de afdeling toxicologie bedanken voor de gezelligheid en de hulp tijdens mijn promotie. Als eerste wil ik Jiska bedanken voor de begeleiding tijdens mijn wetenschapsstage. Bedankt voor alle uitleg, hulp en goede adviezen en alle tijd die je hiervoor nam, ook al was je zelf druk bezig met de afronding van je eigen proefschrift.

Daarnaast wil ik mijn paranimfen, Danielle en Kristien, die me allebei al voor zijn gegaan met hun eigen promotie en me de afgelopen tijd via de app hebben aangemoedigd en adviezen hebben gegeven heel erg bedanken. Danielle, je bent tijdens mijn hele periode op de toxicologie mijn kamergenootje geweest en dat was super gezellig. Op de een of andere manier leek je overal verstand van te hebben en kon ik je altijd alles vragen. Als je zelf het antwoord niet wist, wist je wel iemand die me er dan mee kon helpen. We hebben samen ontzettend veel plezier gehad, zeker ook op onze reis naar Londen samen met Kristien en Lize. Kristien, ik ben ontzettend blij met al je hulp tijdens de proefdierstudie en het feit dat we het samen konden 'ondergaan'. Je was een fijne collega en ik heb erg met je gelachen. Onze gezamenlijke shopping spree door Camden Town, was voor mij toch zeker een van de hoogtepunten 
van de Londen reis. Ik geloof dat we ook nog een congres bezocht hebben... Danielle en Kristien super bedankt dat jullie mijn paranimfen willen zijn!

Hilde, je was maar relatief kort mijn kamergenootje, maar ook jij zorgde voor een warm welkom voor mij op de afdeling toxicologie. Je zorgde voor veel gezelligheid op de kamer en ik heb er veel bewondering voor hoe jij alles rondom je eigen promotie zonder stress leek te regelen, terwijl er tussendoor genoeg tijd was om te kletsen of mee te zingen met de radio. Ook de andere AIOS van de toxicologie wil ik bedanken voor de gezelligheid op de afdeling en tijdens de gezamenlijke activiteiten zoals de etentjes en movie nights. Erik, Agnieszka, Matt (thanks for changing my perception of Americans, crazy American ;)), Thalita, Nuria, Merel en Max bedankt! Roger B bedankt voor het organiseren van de leuke uitjes en voor alle hulp op het lab. Leonie en MarieJosé bedankt voor alle hulp met de proefdierstudie.

Daarnaast alle andere collega's van de toxicologie Antje, Gertjan, Esther, Lou, Danielle P, Joy, Agnes, Roger G, Geja, Kimberly, Marieke, Frederik-Jan, Agnieszka S, Edwin, Jan en Pieter bedankt voor de samenwerking. Ten slotte Marie-Claire bedankt voor al het regelwerk rondom de promotie en de administratieve zaken.

Ik heb tijdens mijn onderzoek ook een aantal studenten mogen begeleiden. Dimosthenis, bedankt voor je medewerking bij het citraat artikel. Kelly ontzettend veel dank voor je hulp bij de metingen voor de proefdier studie. Ook Paulien bedankt, jouw werk hebben we helaas niet meer kunnen verwerken in het proefschrift.

Ik wil ook een aantal collega's van andere afdelingen bedanken voor hun hulp bij verschillende studies. Leo Koehler, Paul van Dijk, Wout Lamers, Sofie Wahlenberg, Ronit Shiri-Sverdlov, Eddie Wisse, Hans Duimel en Jaap Bakker bedankt.

Daarnaast wil ik mijn familie en vrienden bedanken voor de interesse en de nodige afleiding tijdens deze periode. In het bijzonder papa en mama, bedankt dat ik altijd alles heb kunnen doen wat ik wilde doen met onvoorwaardelijke steun van het thuisfront en bedankt voor het altijd trots zijn. Ome Sef, bedankt voor het steeds informeren naar mijn proefschrift wanneer we elkaar zagen. Dat jij graag een exemplaar wilde hebben was toch wel een van de redenen om dat boekje nu eindelijk eens af te maken.

Lieve Niels, bedankt voor je geduld, het aanhoren van mijn frustraties, de aanmoedigingen, de adviezen, het altijd klaar staan en de goede zorgen als ik omkwam in het werk en daarnaast natuurlijk voor alle andere dingen om me van het werk af te 
leiden. Dankzij jou heb ik het proefschrift toch af kunnen schrijven terwijl ik aan een nieuwe opleiding begonnen was. Ik kijk uit naar alle tijd die we nu samen overhouden! 
Curriculum Vitae 
Bregje van de Wier was born on December 25th 1985 in Tilburg, The Netherlands. After finishing secondary school at "Koning Willem II College" in Tilburg, she started with the study Medicine at Maastricht University in 2004. She did her master scientific internship at the department of Pharmacology and Toxicology at Maastricht University, where she investigated the role of cytochrome P450 2E1 in non-alcoholic fatty liver disease. She graduated in October 2010. In December 2010 she started her $\mathrm{PhD}$ project at the department of Toxicology at Maastricht University. The research performed during this project, under supervision of prof. dr. A. Bast, dr. G.R.M.M. Haenen and G.H. Koek, is described in this thesis. In September 2013 she started as physician at "de Riethorst Stromenland" and in March 2014 she started her education as elderly care physician at Radboud University in Nijmegen. 
List of publications 


\section{Full papers}

van de Wier B, Balk JM, Haenen GR, Giamouridis D, Bakker JA, Bast BC, den Hartog GJ, Koek GH, Bast A. Elevated citrate levels in non-alcoholic fatty liver disease: the potential of citrate to promote radical production. FEBS Lett. 2013 Aug 2;587(15):2461-6.

van de Wier B, Koek GH, Bast A, Haenen GR. The Potential of Flavonoids in the Treatment of Non-alcoholic Fatty Liver Disease. Crit Rev Food Sci Nutr. 2015 Apr 21:0. [Epub ahead of print]

van de Wier B, Lemmens KJ, Koek GH, Koehler SE, Wisse E, Duimel H, Shiri-Sverdlov R, Walenbergh S, Bast A, Haenen GRMM. The role of SOD2 in the development of $\mathrm{NASH}$

Lemmens KJ*, van de Wier B*, Koek GH, Köhler E, Drittij MJ, van der Vijgh WJ, Bast A, Haenen GR. The flavonoid monoHER promotes the adaption to oxidative stress during the onset of NAFLD. Biochem Biophys Res Commun. 2015 Jan 2;456(1):179-82.

van de Wier B*, Balk JM*, Bast A, Koek GH, Haenen GR. Chemical characteristics for optimizing CYP2E1 inhibition. Chem Biol Interact. 2015 Dec 5;242:139-44.

Lemmens KJ, van de Wier B, Vaes N, Ghosh M, van Zandvoort MA, van der Vijgh WJ, Bast A, Haenen GR. The flavonoid 7-mono-0-( $\beta$-hydroxyethyl)-rutoside is able to protect endothelial cells by a direct antioxidant effect. Toxicol In Vitro. 2014 Jun;28(4):538-43.

\section{Abstracts}

van de Wier B, Balk J.M., Giamouridis D., Bakker J. A., Koek G.H., Haenen G.R.M.M., Bast A. Increase of oxidative stress in NASH by elevated levels of citrate. Society for Free Radical Research International meeting, September 2012, London, UK

* equal contribution 


\section{List of abbreviations}


A $\mathrm{ACC}$

ALT

AMPK

ARES

ASH

AST

B BMI

C CAP

CAT

CB1 receptor

CB2 receptor

CDNA

ChREBP

CK18

COX

CT

CYP2E1

D DAMPS

DCFH-DA

Deoxyribose

DMPO

DPBS

DTNB

E EGCG

ER

ESR

ETC

F FAS

$\mathrm{Fe}^{2+}$

$\mathrm{FeCl}_{3}$

$\mathrm{FeSO}_{4}$

FFA

FLI

G $\quad \gamma G T$

Gapdh

Gclc

gk

GPx

GSH

GSSG

GTE

H HCC

HDL

HepG2

HFC

Hmox1

HO-1

$\mathrm{H}_{2} \mathrm{O}_{2}$

I IDL

IKB

IKK
acetyl-CoA carboxylase

alanine aminotransferase

$5^{\prime}$ adenosine monophosphate-activated protein kinase

antioxidant response elements

alcoholic steatohepatitis

aspartate aminotransferase

body mass index

controlled attenuation parameter

catalase

cannabinoid receptor type-1

cannabinoid receptor type-2

complementary DNA

carbohydrate response element binding protein

cytokeratin 18

cyclooxygenase

computed tomography

cytochrome P450 2E1

damage-associated molecular patterns

2,7-dichlorofluorescin diacetate

2-deoxy-D-ribose

5-diethoxyphosphoryl-5-methyl-1-pyrroline $\mathrm{N}$-oxide

Dulbecco's phosphate buffered saline

5,5-dithio-bis-(2-nitrobenzoic acid)

epigallocatechin-gallate

endoplasmic reticulum

electron spin resonance

electron transport chain

fatty acid synthase

ferrous ion

ferric chloride

ferrous sulphate

free fatty acids

fatty liver index

gamma-glutamyl transpeptidase

glyceraldehyde-3-phosphate dehydrogenase gene

glutamate-cysteine ligase catalytic subunit gene

glucokinase

glutathione peroxidase

glutathione

glutathione disulfide

green tea extract

hepatocellular carcinoma

high density lipoprotein

human hepatoma cell line

high fat high cholesterol

heme oxygenase 1 gene

heme oxygenase 1

hydrogen peroxide

intermediate density lipoproteins

$\mathrm{NFKB}$ inhibitor protein

IKB kinase complex 


\begin{tabular}{|c|c|c|}
\hline & IL & interleukin \\
\hline & iNOS & nitric oxide synthase \\
\hline K & KEAP1 & Kelch-like ECH-associated protein 1 \\
\hline $\mathbf{L}$ & LDL & low density lipoprotein \\
\hline & LDLr & low density lipoprotein receptor \\
\hline & LPS & lipopolysaccharide \\
\hline & LXR $\alpha$ & liver X-receptor $\alpha$ \\
\hline M & MCD & Methionine choline deficient \\
\hline & MDA & malondialdehyde \\
\hline & MonoHER & 7-mono-O-( $\beta$-hydroxyethyl)-rutoside \\
\hline & MRI & magnetic resonance imaging \\
\hline & MRS & magnetic resonance spectroscophy \\
\hline & mt-DNA & mitochondrial DNA \\
\hline $\mathbf{N}$ & $\mathrm{NADH}$ & nicotinamide dinucleotide \\
\hline & NADP+ & nicotinamide adenine dinucleotide phosphate \\
\hline & NAFLD & non-alcoholic fatty liver disease \\
\hline & NAS & NAFLD activity score \\
\hline & NASH & non-alcoholic steatohepatitis \\
\hline & NFDM & non-fat dry milk \\
\hline & NF-kB & nuclear factor kappa B \\
\hline & NO & nitric oxide \\
\hline & Nqo1 & $\mathrm{NAD}(\mathrm{P}) \mathrm{H}$ dehydrogenase, quinone 1 gene \\
\hline & NRF2 & nuclear factor erythroid derived 2 \\
\hline 0 & $\mathrm{O}_{2}^{\cdot-}$ & superoxide anion radical \\
\hline & ${ }^{\circ} \mathrm{OH}$ & hydroxyl radical \\
\hline $\mathbf{P}$ & PKC & protein kinase $\mathrm{C}$ \\
\hline & PPAR & peroxisome proliferator activated receptor \\
\hline $\mathbf{R}$ & ROS & reactive oxygen species \\
\hline $\mathbf{S}$ & SOD & superoxide dismutase \\
\hline & $\begin{array}{l}\text { SOD2- } \mathrm{KO}_{\mathrm{L}} \text { mice } \\
\text { SREBP-1C }\end{array}$ & $\begin{array}{l}\mathrm{SOD} 2^{\mathrm{fl} / \mathrm{fl}} / \mathrm{Alb}^{\mathrm{C}} \mathrm{Cre}{ }^{\mathrm{tg} /-} \text { mice with a liver-specific SOD2 knock-out } \\
\text { sterol regulatory element binding protein-1c }\end{array}$ \\
\hline $\mathbf{T}$ & TCA & tri-chloro-acetic acid \\
\hline & TEAC & trolox equivalent antioxidant capacity \\
\hline & TEM & transmission electron microscopy \\
\hline & TG & triglycerides \\
\hline & TNB & 5'-thio-2-nitrobenzoic acid \\
\hline & TNF- $\alpha$ & tumor necrosis factor $\alpha$ \\
\hline & TLRs & toll-like receptors \\
\hline $\mathbf{u}$ & UDCA & ursodeoxycholic acid \\
\hline $\mathbf{v}$ & VLDL & very low density lipoprotein \\
\hline w & WT & wildtype \\
\hline $\mathbf{Y}$ & Ywhaz & $\begin{array}{l}\text { tyrosine 3-monooxygenase/tryptophan 5-monooxygenase } \\
\text { activation protein, zeta polypeptide }\end{array}$ \\
\hline
\end{tabular}





\section{Supplementary Data}




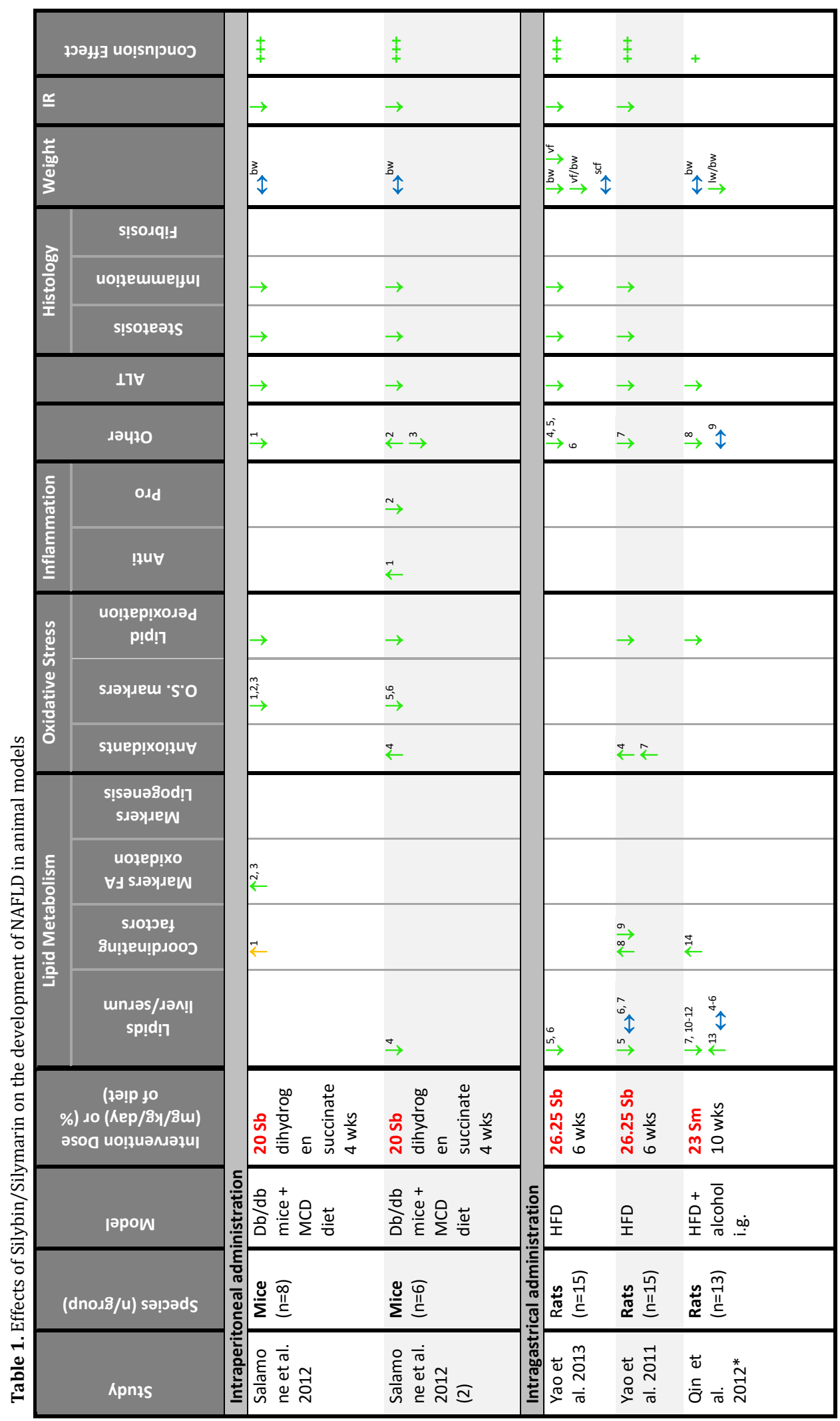




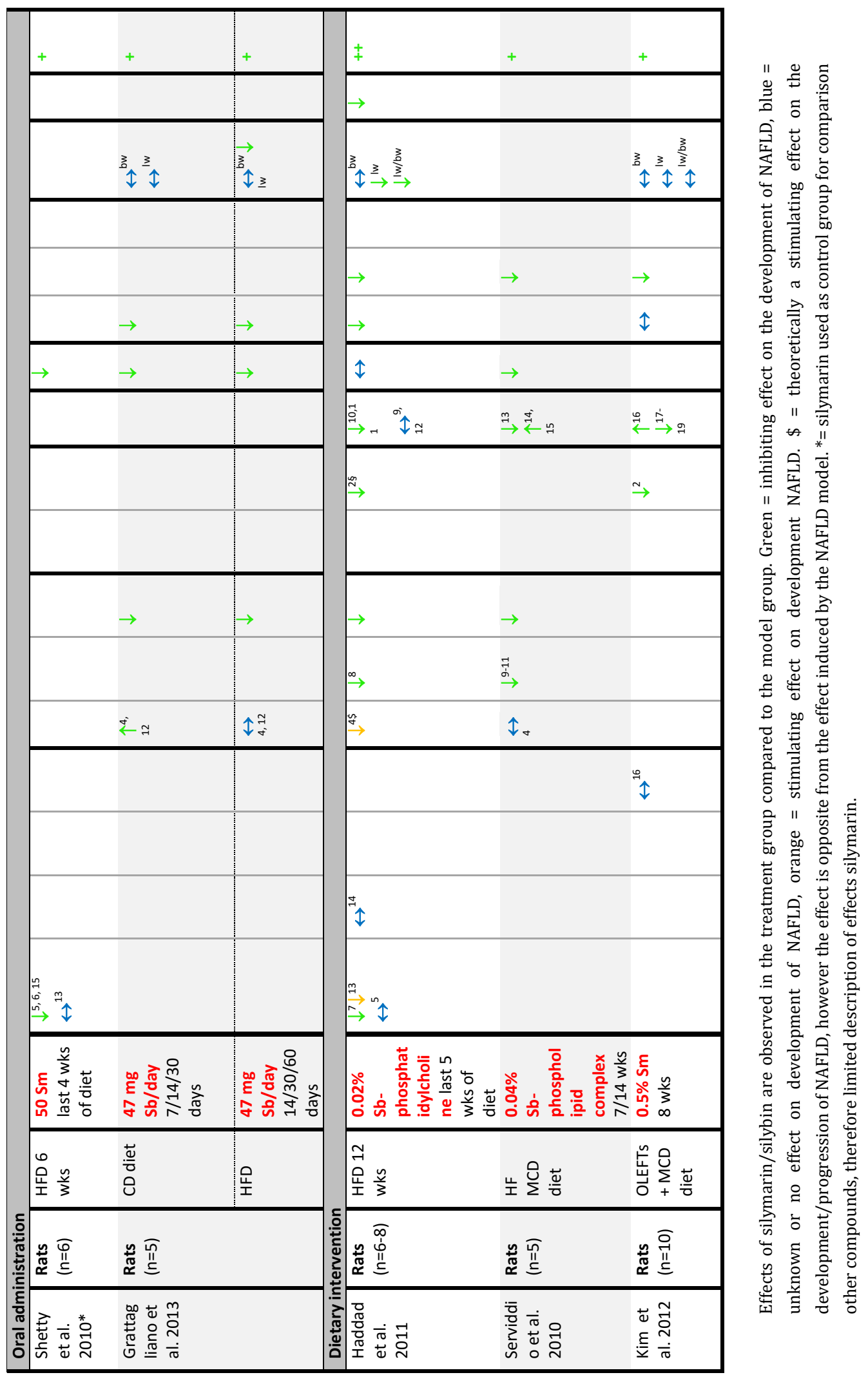




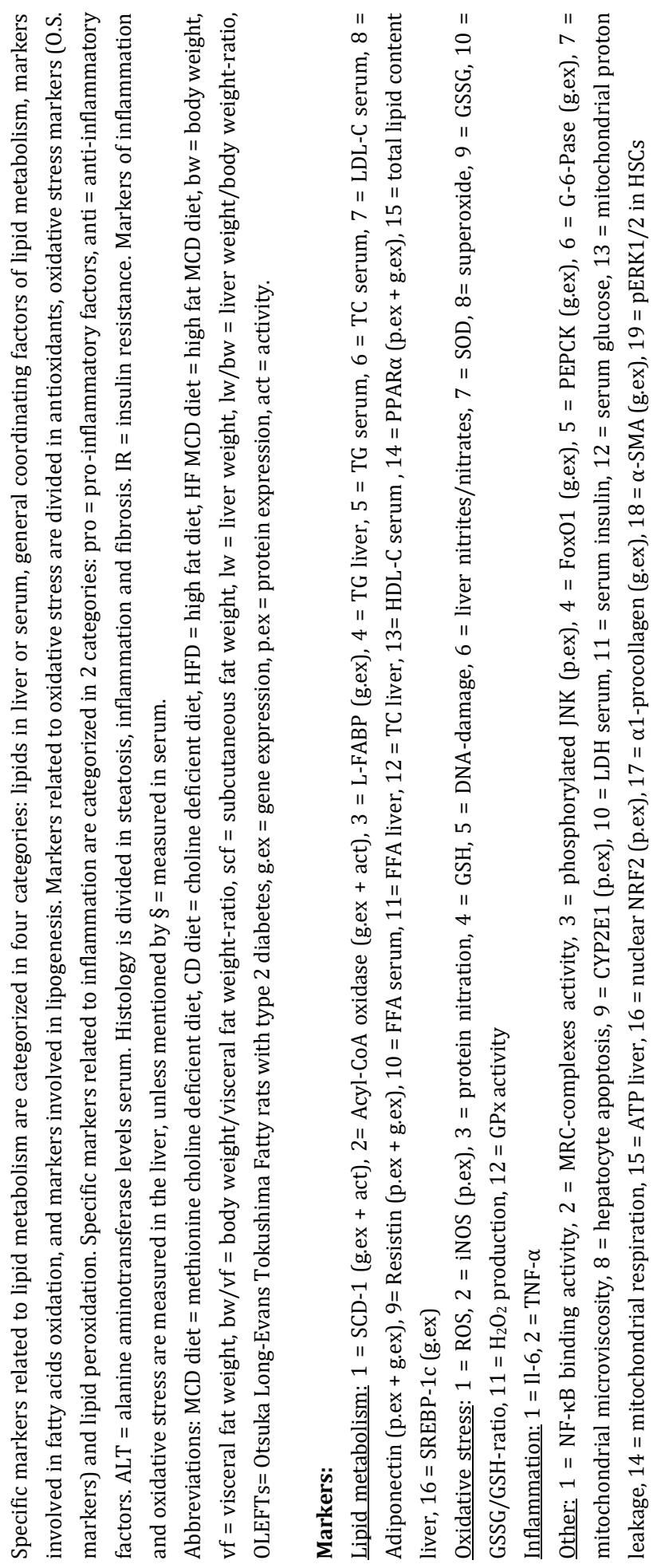




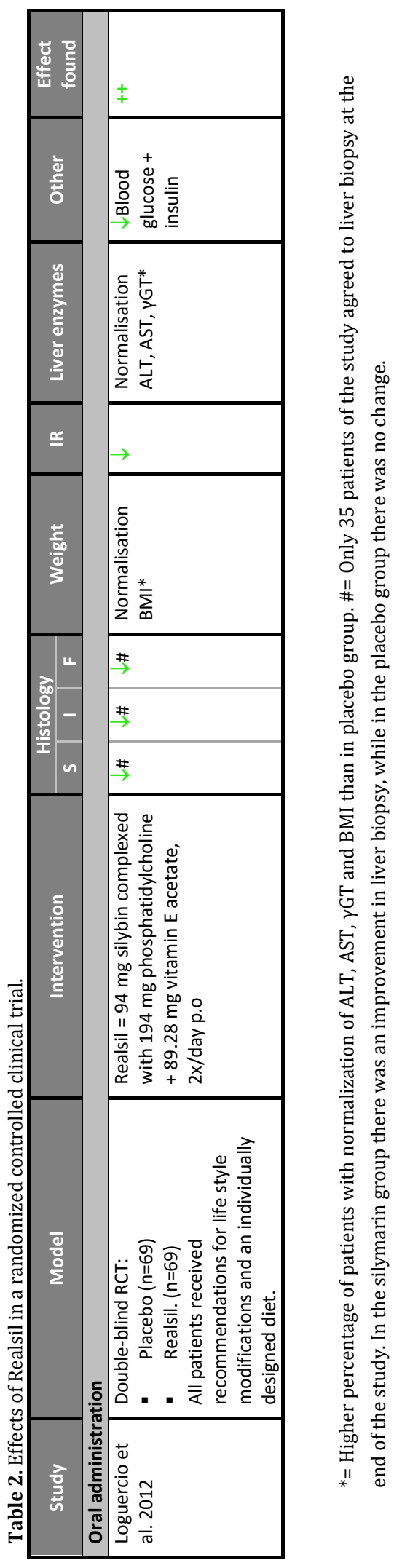




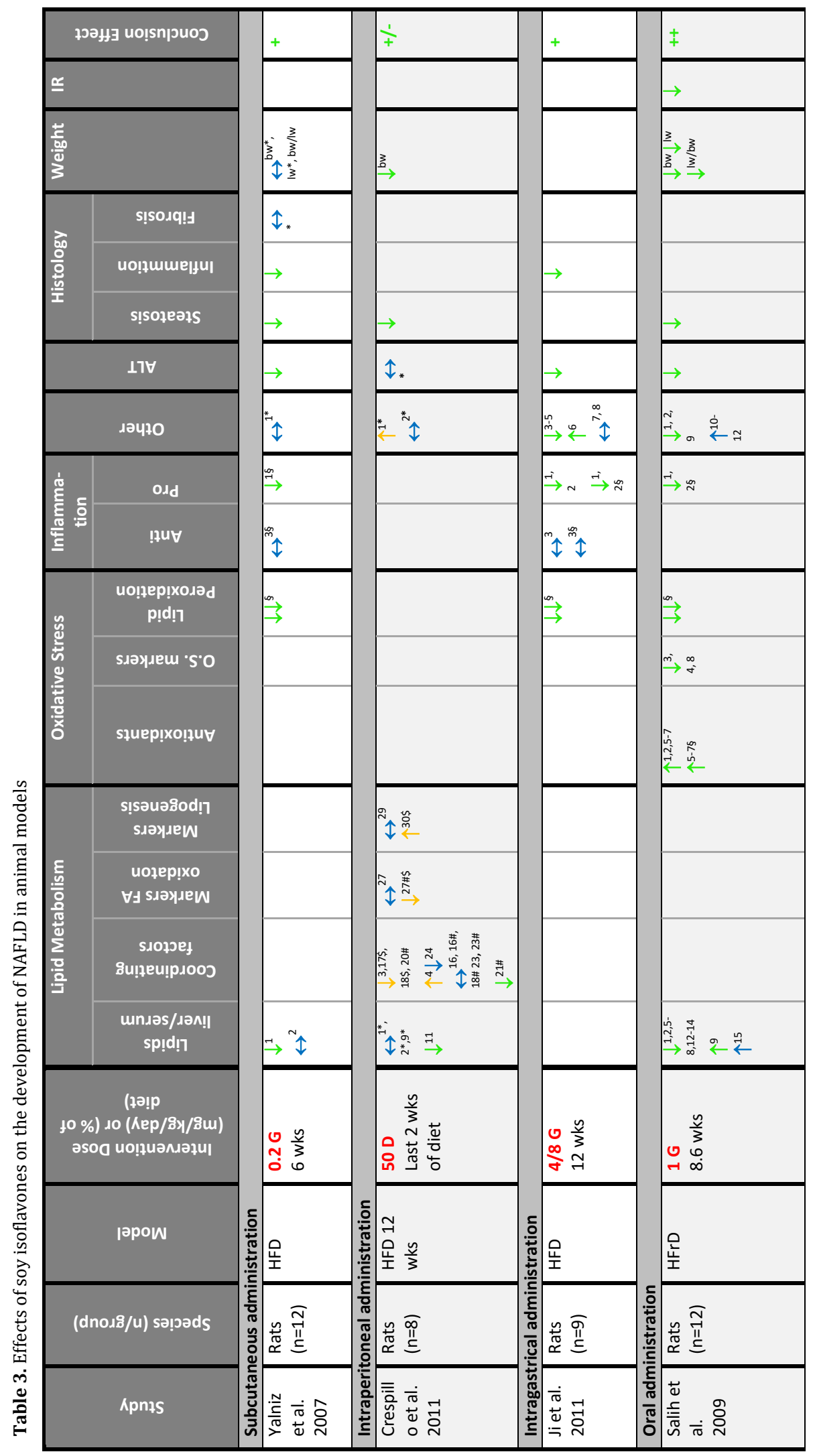




\begin{tabular}{|c|c|c|c|c|c|c|}
\hline & + & + & $\stackrel{1}{+}$ & $\stackrel{1}{+}$ & $\stackrel{1}{+}$ & + \\
\hline & & & $\rightarrow$ & $\rightarrow$ & & \\
\hline & $\stackrel{3}{\rightarrow} \stackrel{\frac{3}{5}}{\rightarrow}$ & $\stackrel{\overrightarrow{3}}{\overrightarrow{3}} \underset{\stackrel{3}{\rightarrow}}{\rightarrow}$ & $\stackrel{\imath}{1} 3$ & $\stackrel{3}{\rightarrow} \stackrel{3}{\rightarrow} \stackrel{3}{\rightarrow} \stackrel{3}{\uparrow}$ & & $\stackrel{3}{\rightarrow}$ \\
\hline & $\rightarrow$ & $\rightarrow$ & & $\rightarrow$ & $\rightarrow$ & \\
\hline & $\rightarrow$ & & & $\rightarrow$ & $\rightarrow$ & \\
\hline & & & 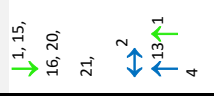 & $\stackrel{\substack{ज \\
\rightarrow}}{\rightarrow} \tilde{Z}$ & & $\stackrel{\sim}{\vec{\Xi}}_{m}$ \\
\hline & $\rightarrow_{\rightarrow}$ & & & $\stackrel{\rightrightarrows}{\rightarrow} \stackrel{-}{\rightarrow}$ & & $\stackrel{\rightarrow}{\rightarrow} \stackrel{-i}{\rightarrow}$ 的 \\
\hline & & $\uparrow$ & & & & \\
\hline & & 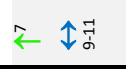 & & $\stackrel{\approx}{\leftarrow} \stackrel{m}{\leftarrow}$ & & \\
\hline & 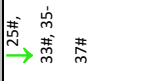 & & 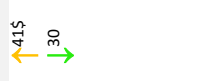 & $\stackrel{m}{\leftarrow} \uparrow_{\Omega} \stackrel{\infty}{\rightarrow}$ & 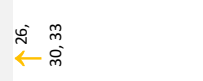 & \\
\hline & $\begin{array}{l}\text { 范 } \\
\text { L } \\
\end{array}$ & $\stackrel{m}{1}+\frac{i}{1}$ & 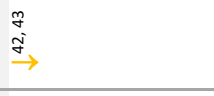 & 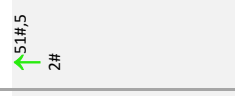 & 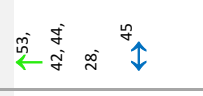 & \\
\hline & 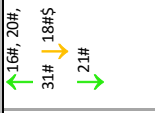 & 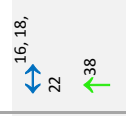 & $+\uparrow$ & 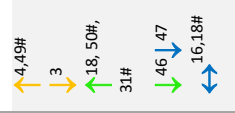 & 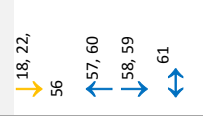 & \\
\hline & 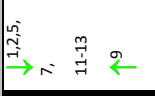 & 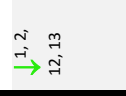 & 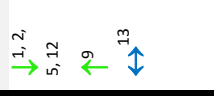 & 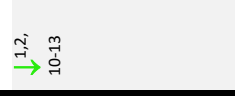 & 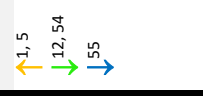 & $\stackrel{\cong}{\rightarrow}$ \\
\hline & 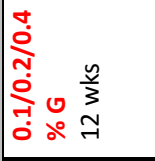 & 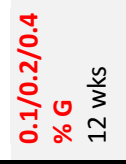 & 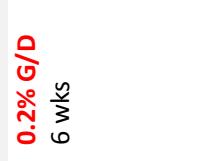 & 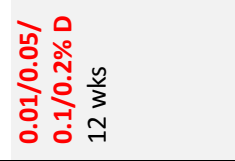 & 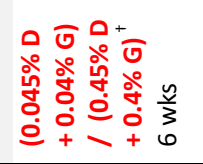 & 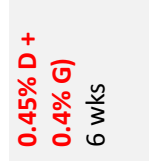 \\
\hline & 虫 & 殅 & $\frac{0}{\frac{0}{0}} . \stackrel{0}{\frac{u}{\varepsilon}}$ & 岂 & 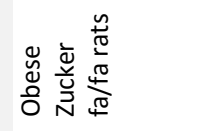 & 岕产 \\
\hline 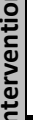 & 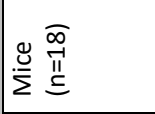 & 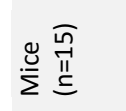 & 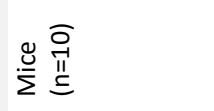 & 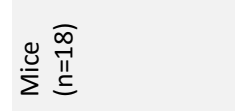 & 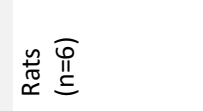 & 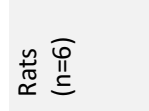 \\
\hline$\frac{2}{0}$ & 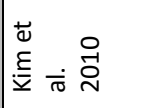 & $\begin{array}{l}\longleftarrow \\
\Xi \\
\Xi\end{array}$ & 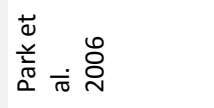 & 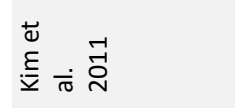 & 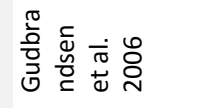 & 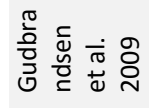 \\
\hline
\end{tabular}



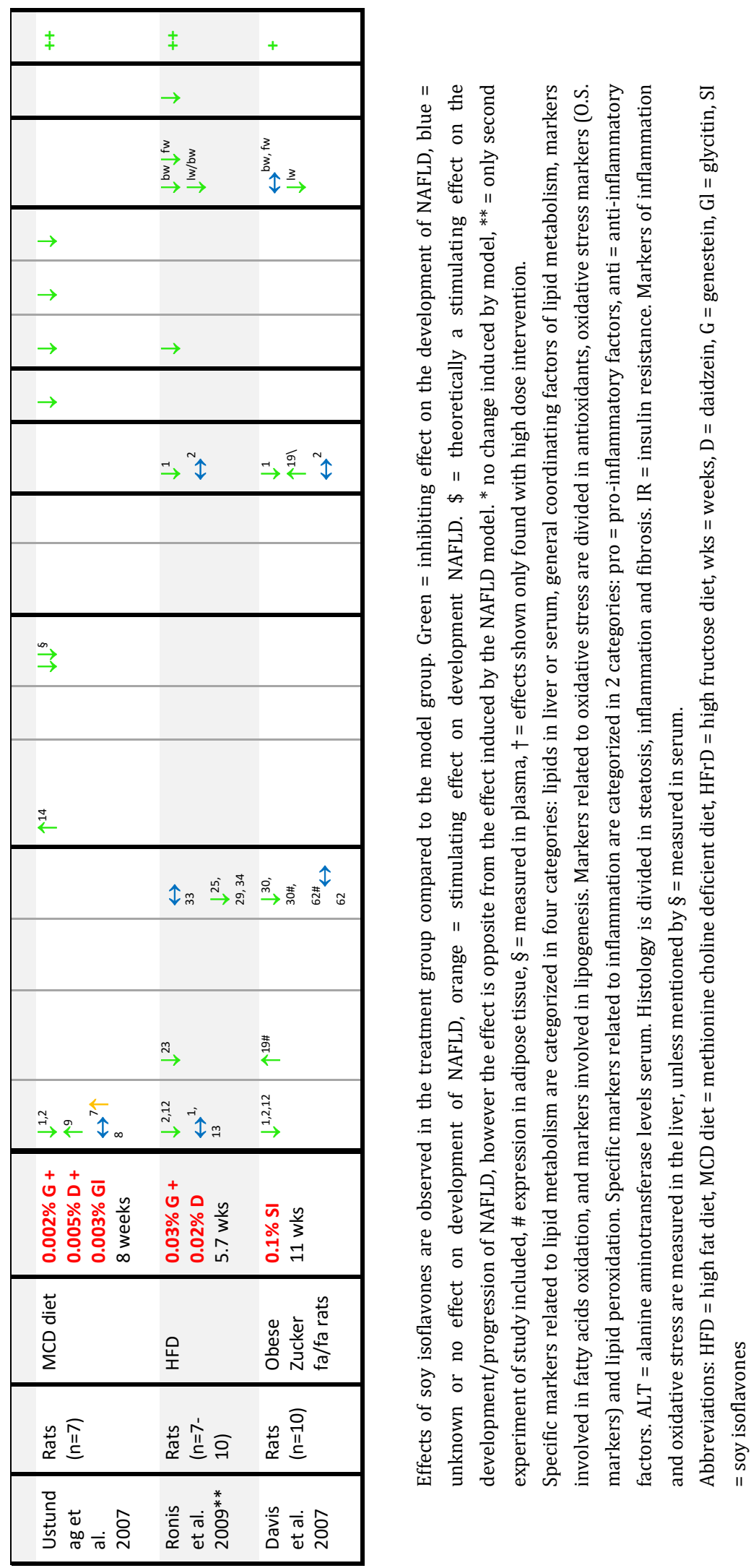


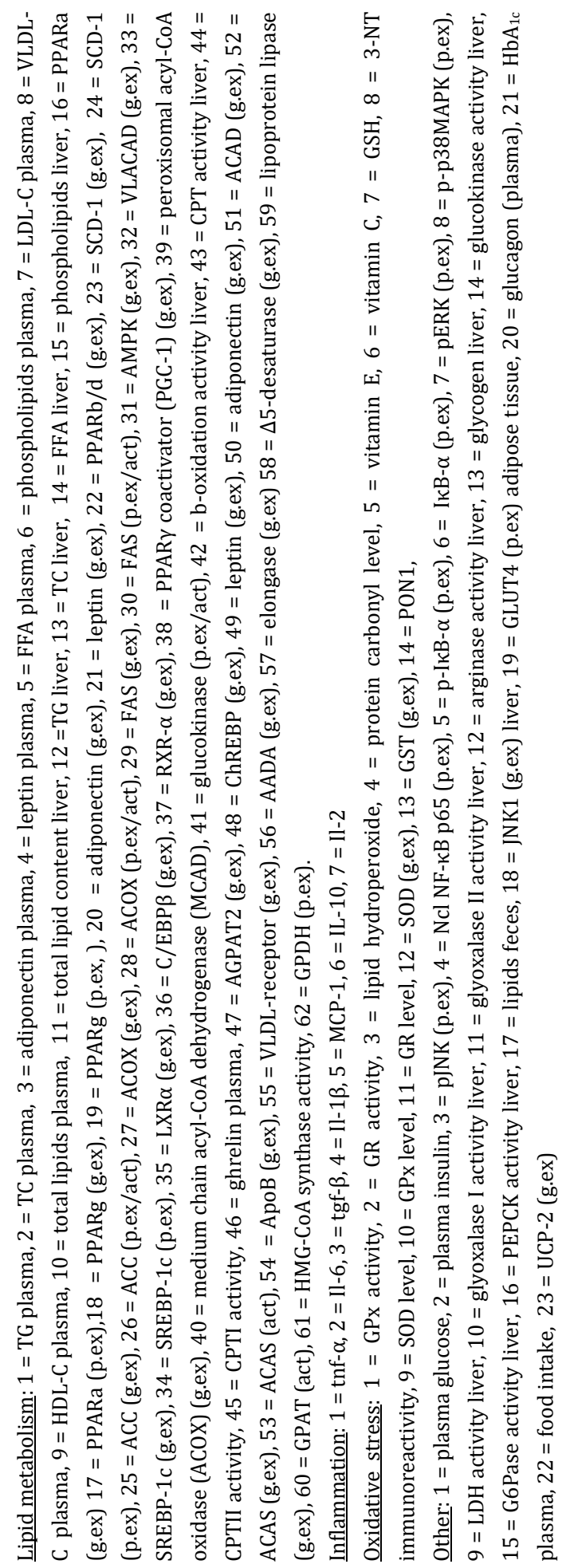




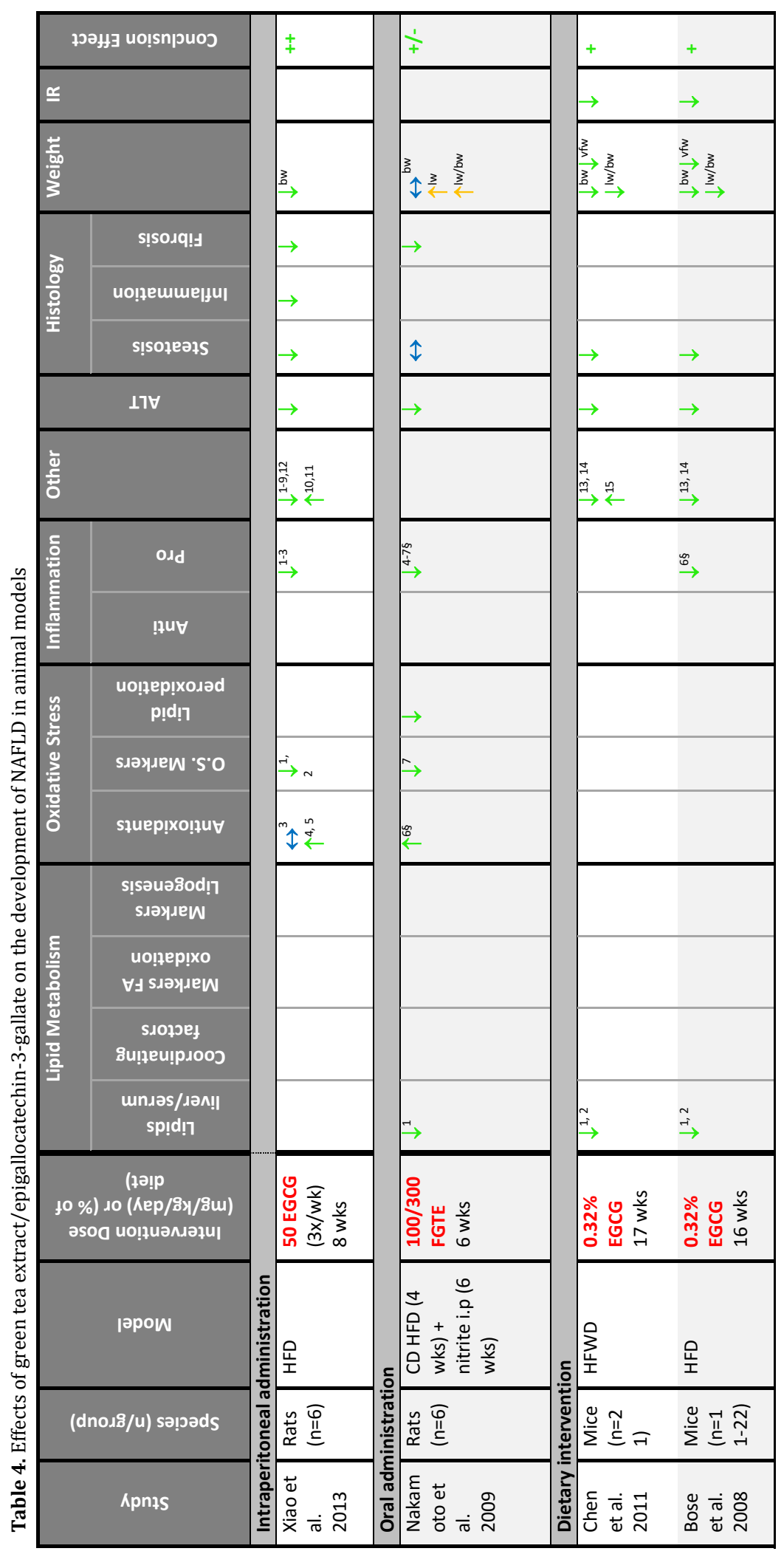




\begin{tabular}{|c|c|c|c|c|c|}
\hline+ & + & + & + & + & + \\
\hline \multicolumn{6}{|l|}{$\rightarrow$} \\
\hline$\stackrel{3}{1} \stackrel{3}{\longrightarrow}$ & $\stackrel{3}{1} \stackrel{3}{\underline{3}}$ & $\stackrel{\stackrel{3}{\rightarrow}}{\stackrel{\overrightarrow{3}}{\rightarrow}}$ & $\stackrel{\stackrel{ \pm}{\vec{B}}}{\rightarrow} \stackrel{3}{\rightarrow}$ & $\stackrel{\stackrel{3}{\rightarrow}}{\rightarrow}$ & $\stackrel{\stackrel{3}{\longrightarrow}}{\stackrel{3}{\rightarrow}}$ \\
\hline & & & & $\rightarrow$ & \\
\hline$\rightarrow$ & $\rightarrow$ & $\rightarrow$ & & $\rightarrow$ & $\rightarrow$ \\
\hline$\rightarrow$ & $\rightarrow$ & & $\rightarrow$ & $\rightarrow$ & $\rightarrow$ \\
\hline 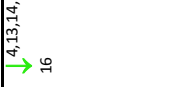 & 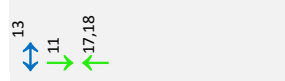 & & & & \\
\hline & & & $\stackrel{\sim}{\rightarrow}$ & $\stackrel{\stackrel{9}{\rightarrow}}{\rightarrow}$ & $\begin{array}{l}\stackrel{m}{i} \\
\stackrel{7}{\rightarrow} \\
\infty\end{array}$ \\
\hline$\rightarrow$ & & & $\rightarrow$ & $\rightarrow$ & \\
\hline & & & & $\stackrel{-\vec{I}}{\rightarrow} \underset{\sim}{\mathrm{N}} \approx$ & \\
\hline$\stackrel{\infty}{\leftarrow}$ & & $\stackrel{9}{\rightarrow} \uparrow$ & 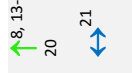 & & $\stackrel{\infty}{\leftarrow}$ \\
\hline & & & $\stackrel{\AA}{\downarrow}$ & & \\
\hline & & 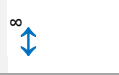 & 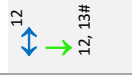 & & $\stackrel{\Delta}{\rightarrow}$ \\
\hline$\stackrel{n}{\imath} \rightarrow$ & $\stackrel{+}{\stackrel{n}{\rightarrow}} \stackrel{\imath}{\rightarrow}$ & $\stackrel{\stackrel{0}{\rightarrow}}{\rightarrow} \curvearrowright \uparrow$ & 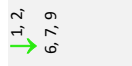 & & $\stackrel{0}{\stackrel{-}{\rightarrow}}$ \\
\hline 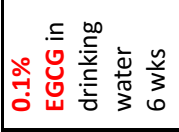 & 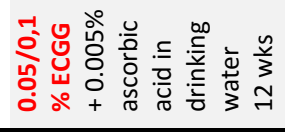 & 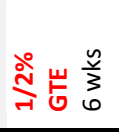 & 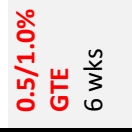 & 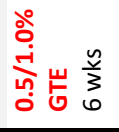 & 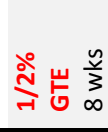 \\
\hline 至 & 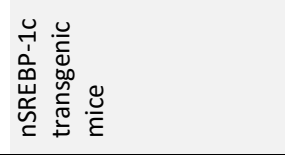 & 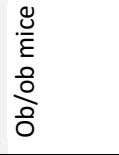 & 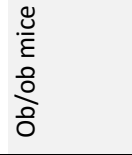 & $\begin{array}{l}\stackrel{\mathscr{v}}{\varepsilon} \\
\stackrel{0}{0} \\
\frac{0}{0}\end{array}$ & 堊 \\
\hline 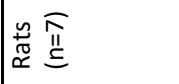 & $\stackrel{\stackrel{\vartheta}{\Sigma}}{\Sigma} \stackrel{0}{=}$ & 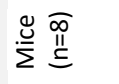 & $\stackrel{\stackrel{\Perp}{\Sigma}}{\Sigma} \stackrel{-1}{=} \widehat{N}$ & 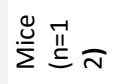 & 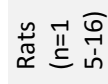 \\
\hline 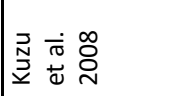 & 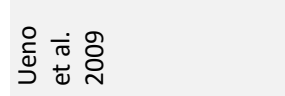 & 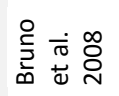 & 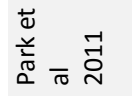 & 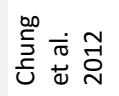 & 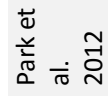 \\
\hline
\end{tabular}




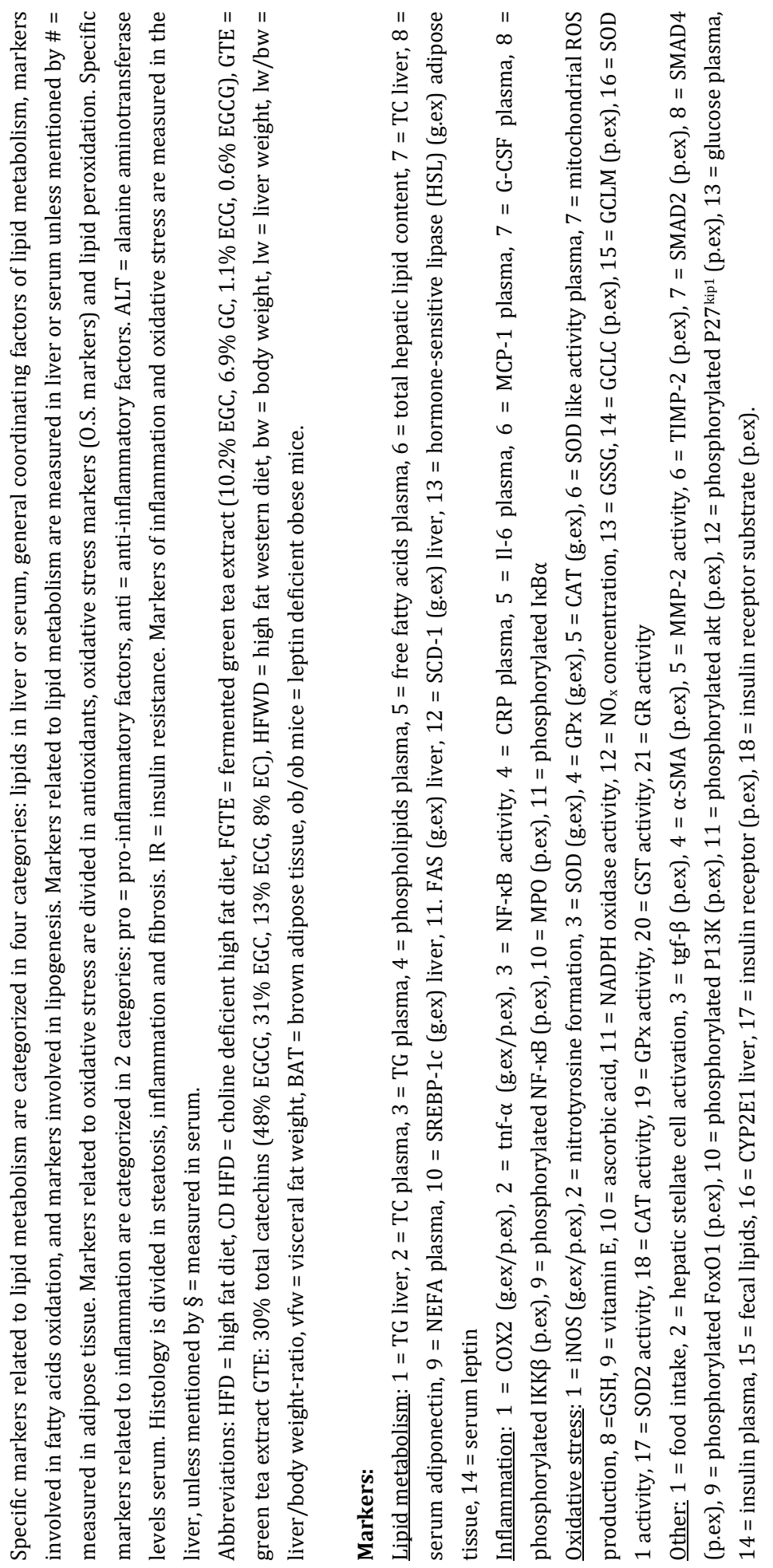





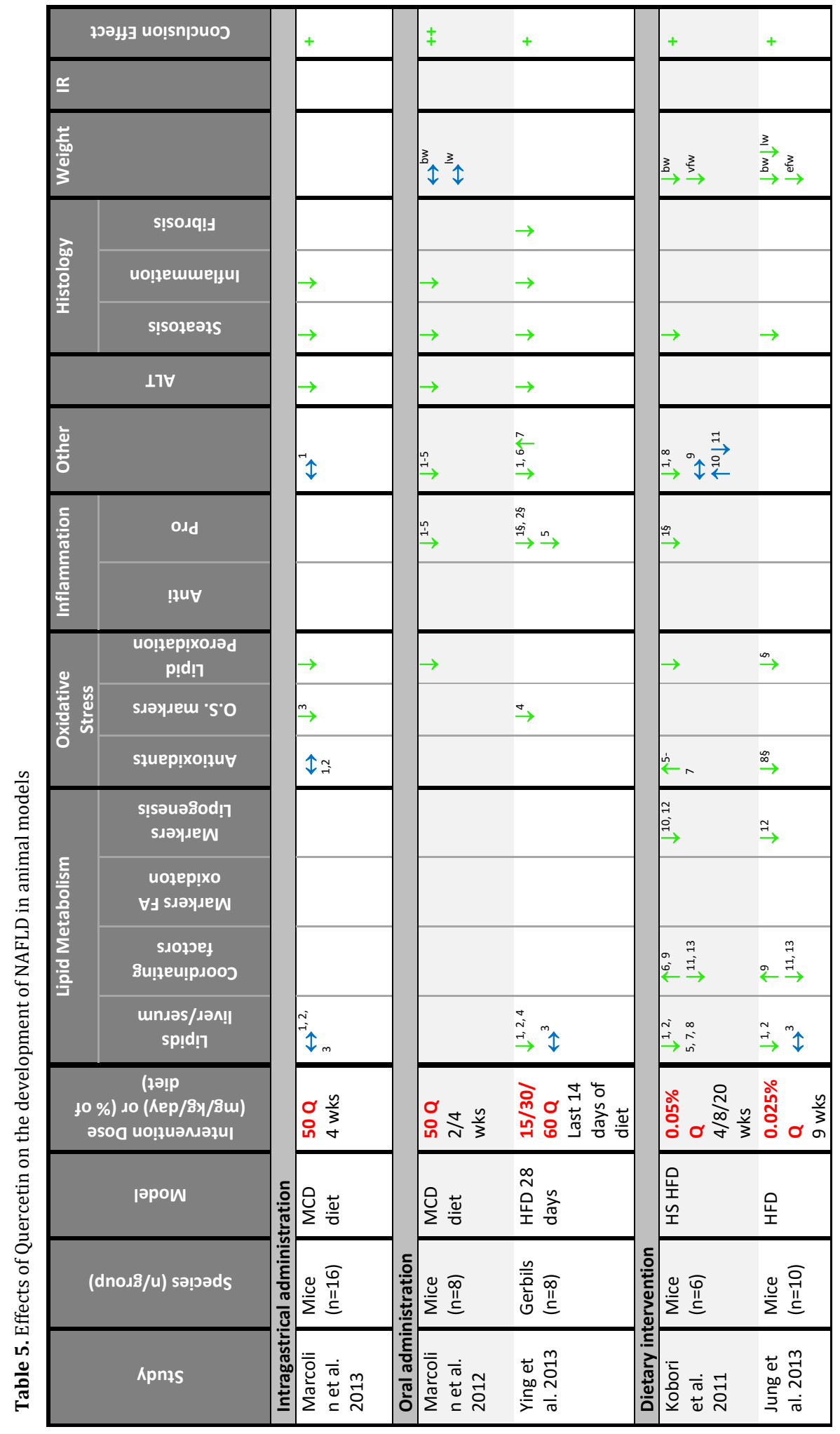




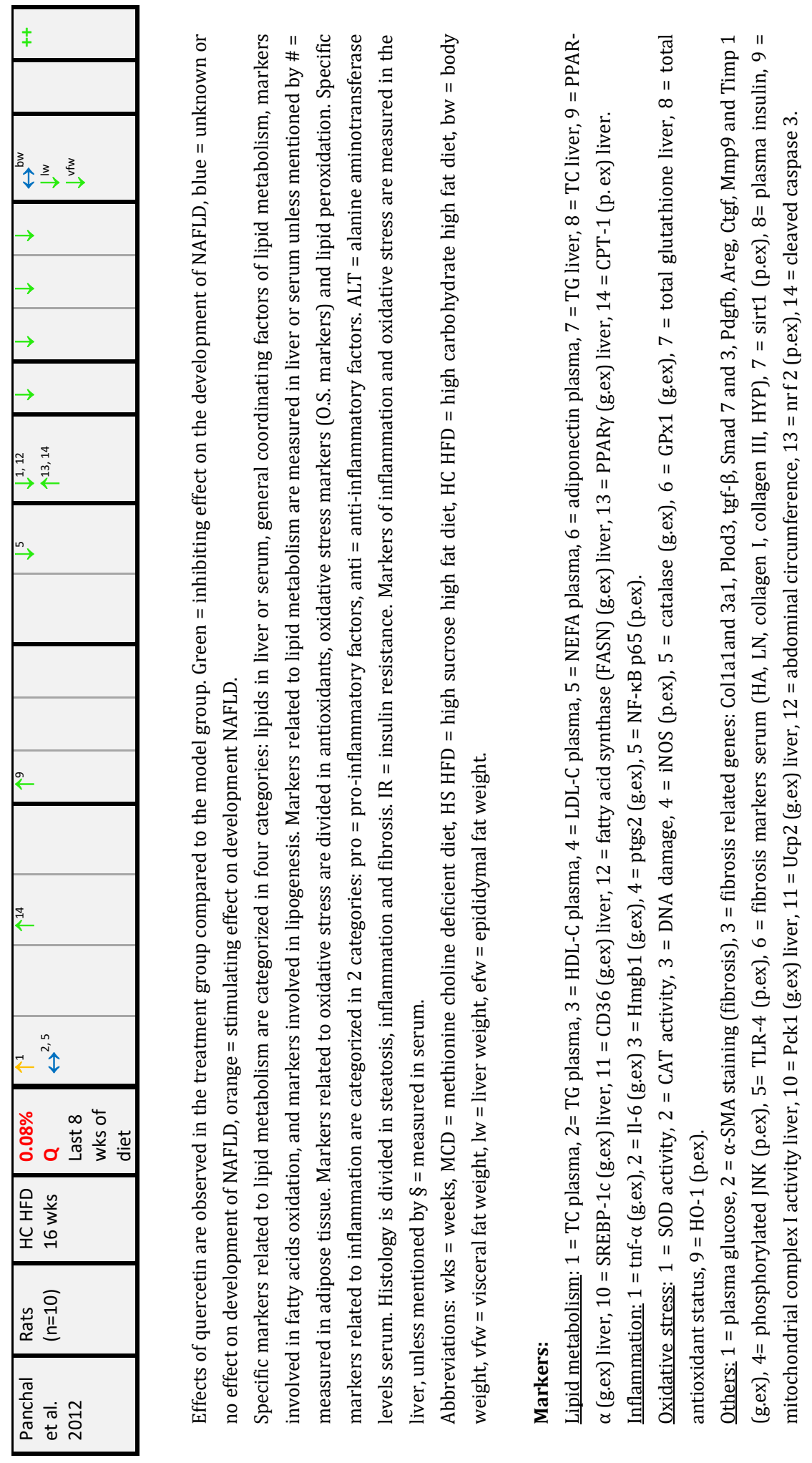




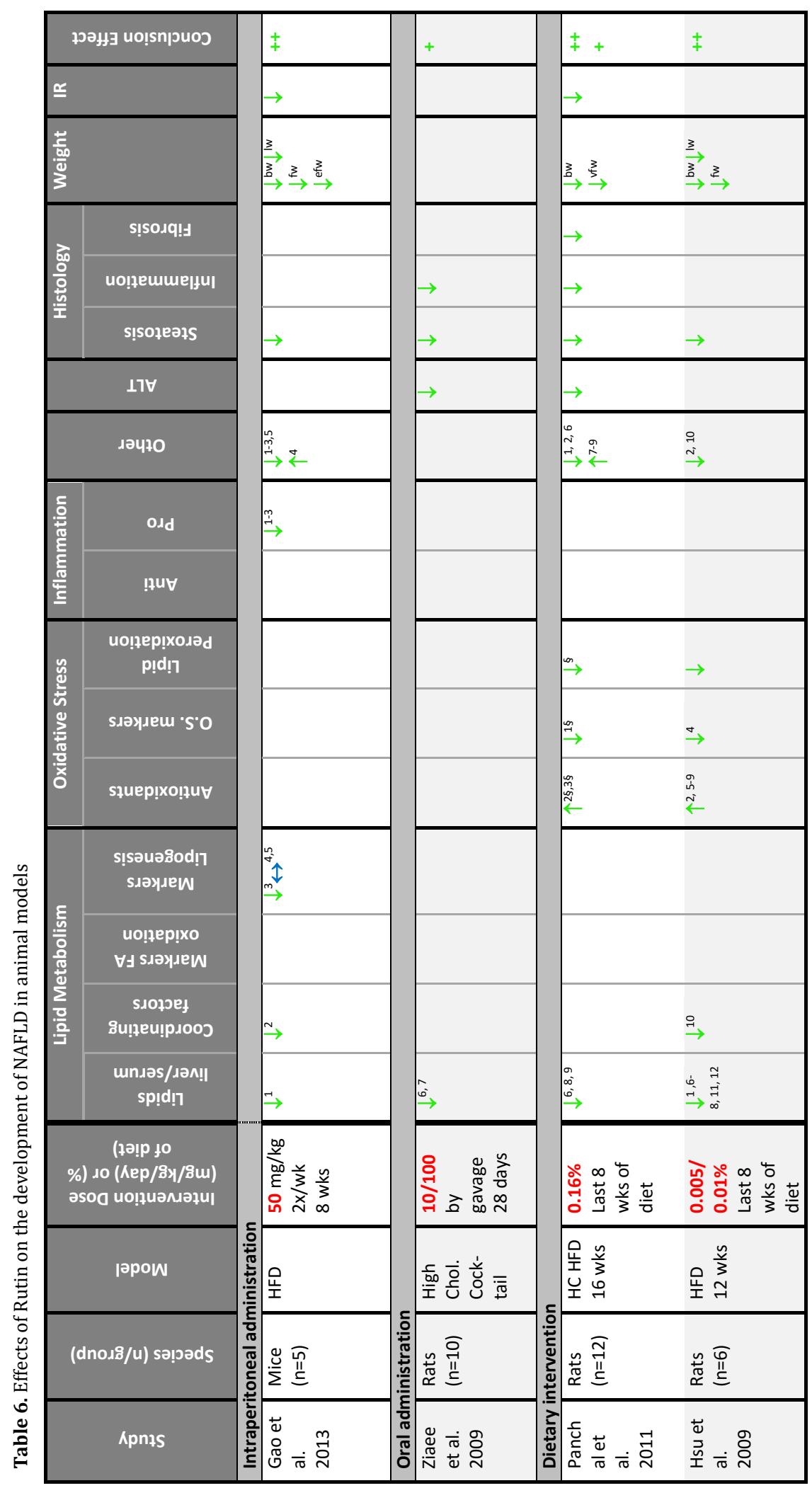




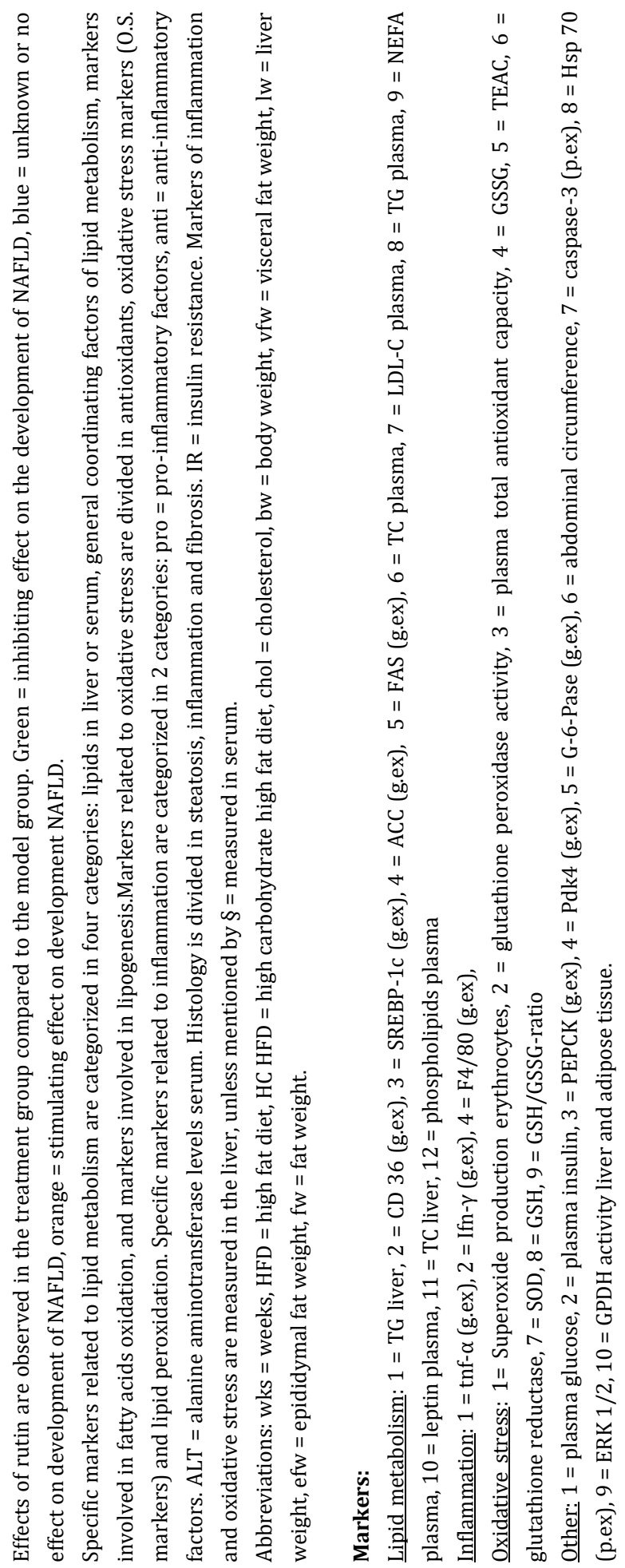


Table 7. Other flavonoids investigated in animal models of NAFLD

\begin{tabular}{|c|c|}
\hline Flavanones & s: \\
\hline Anthocyani & $\begin{array}{l}\text { ins: } \\
\text { Cyanidin-3-O- } \beta-D-\text { glucoside [165-167] }\end{array}$ \\
\hline Chalcones: & Xanthohumol $[168,169]$ \\
\hline $\begin{array}{c}\text { Flavones: } \\
\bullet \\
\bullet\end{array}$ & $\begin{array}{l}\text { Baicalin [170] } \\
\text { Nobiletin [171] }\end{array}$ \\
\hline Isoflavones & $\begin{array}{l}\text { s: } \\
\text { Puerin [173] }\end{array}$ \\
\hline Isoflavanes & S: \\
\hline
\end{tabular}


